\title{
AN ENERGY SAVING MODEL FOR THE HEAT TREATMENT OF CASTINGS
}

Final Report

Worcester Polytechnic Institute 100 Institute Road

Worcester, MA 01609-2280

$12.29,2006$ 
Project Title: $\quad$ An Energy Saving Model for the Heat Treatment of Castings

DOE Award Number: DE-FC36-01ID14197

Project Period: $\quad$ 10:01 - 09:06

Principal

Investigator(s):

Yiming (Kevin) Rong, (508) 831-6020, rong@wpi.edu

Richard D. Sisson, Jr., (508) 831-5335, sisson@wpi.edu

John E. Morral, (614) 292-6255, morral.4@,osu.edu

Harold D. Brody, (860) 486-0853, Harold.Brody@uconn.edu

Recipient: Worcester Polytechnic Institute

100 Institute Road

Worcester, MA 01609-2280

Subcontractors: University of Connecticut

The Ohio State University

Project Team: $\quad$ Dibyajyoti Aichbhaumik (303) 275-4763, debo.aichbhaumik@go.doe.gov 


\section{DOCUMENT AVAILABILITY:}

"Reports are available free via the U.S. Department of Energy (DOE) Information Bridge:

Web Site http://www.osti.gov/bridge

Reports are available to DOE employees, DOE contractors, Energy Technology Data Exchange(ETDE) representatives, and Informational Nuclear Information System (INIS) representatives from the following source:

Office of Scientific and Technical Information

P.O. Box 62

Oak Ridge, TN 37831

Tel: (865) 576-8401

Fax: (865) 576-5728

E-mail: reports@osti.gov

Web Site: http://www.osti.gov/contact.html

Acknowledgment: "This report is based upon work supported by the U. S. Department of Energy under Award No. DE-FC36-01ID14197 “.

Disclaimer: "Any findings, opinions, and conclusions or recommendations expressed in this report are those of the author(s) and do not necessarily reflect the views of the Department of Energy" 


\section{Table of Contents}

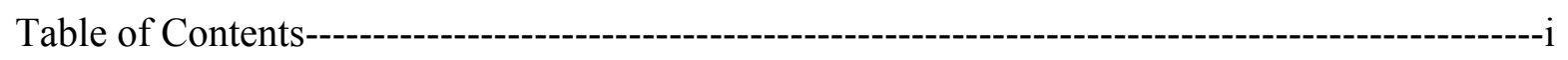

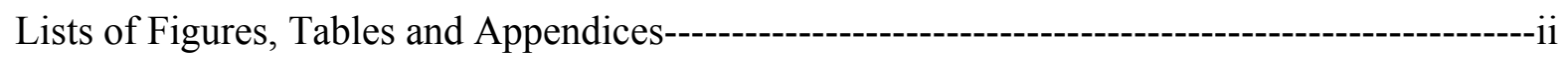

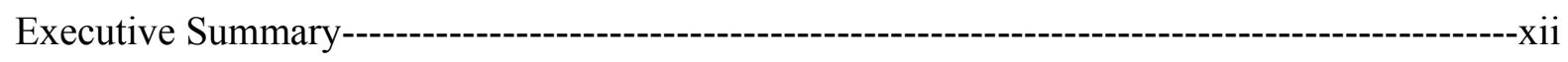

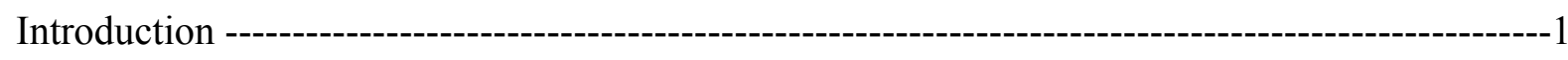

Section 1: Thermal Prediction and optimization----------------------------------------------------------3

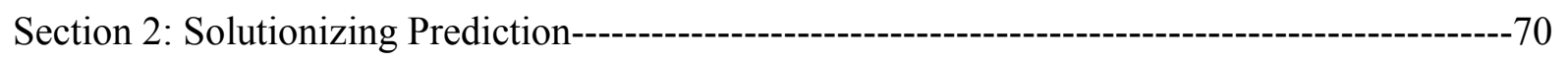

Section 3: Databases------------------------------------------------------------------------------------------136

Section 4: Integration of Thermal-Microstructure-Property Prediction -----------------------------186

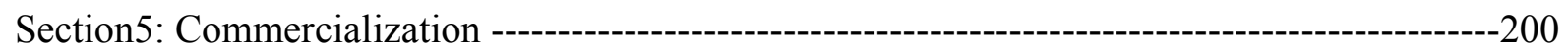

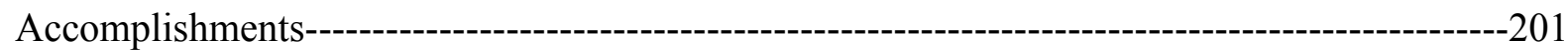

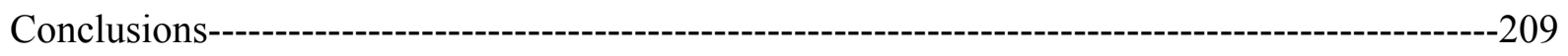

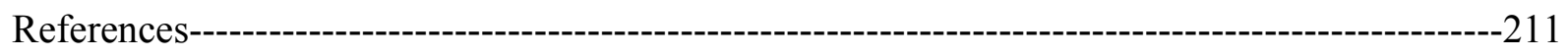




\section{Lists of Figures, Tables and Appendices}

Figure 1.1-1. The technical route of modeling of heat transfer in heat treatment furnace ------- 5

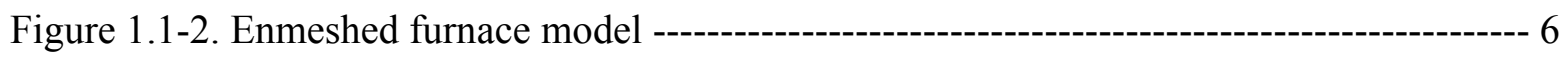

Figure 1.1-3. Thermal resistance between different type materials ---------------------------------- 7

Figure 1.1-4. 2-D illustrative diagram of view factor calculation ------------------------------------ 9

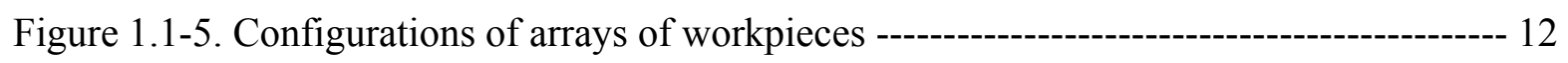

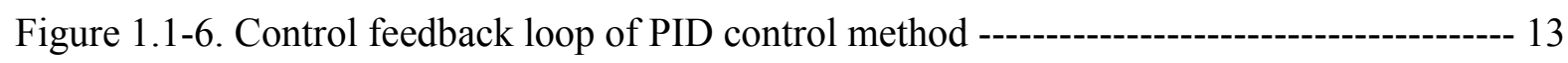

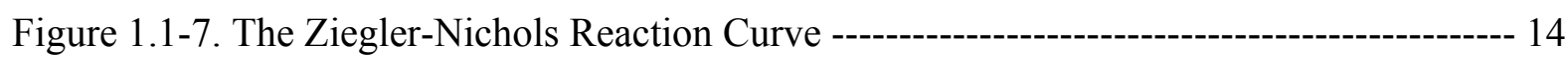

Figure 1.1-8. The system interface ----------------------------------------------------------------------- 15

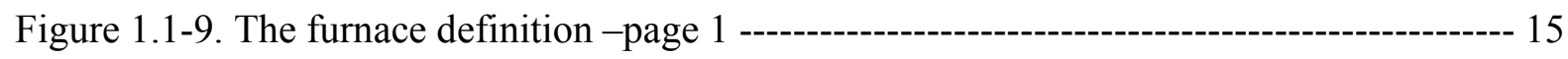

Figure 1.1-10. The furnace definition -page 2 ---------------------------------------------------------- 16

Figure 1.1-11. Thermal schedule dialog -------------------------------------------------------------------- 16

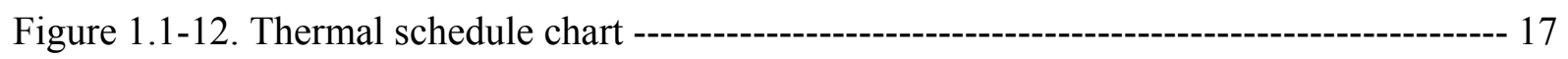

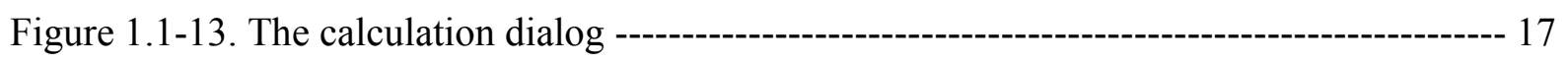

Figure 1.1-14. Geometrical model (partial; Furnace wall, heating elements, parts) -------------- 18

Figure 1.1-15. Inside of FDM model (partial; Furnace wall, heating elements, parts) ----------- 18

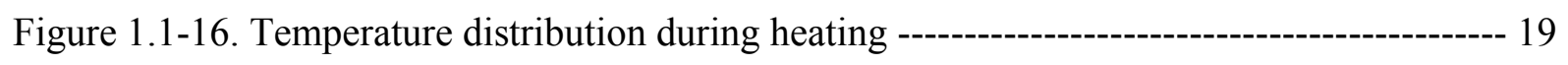

Figure 1.1-17. Temperature distribution in parts during heating ------------------------------------- 19

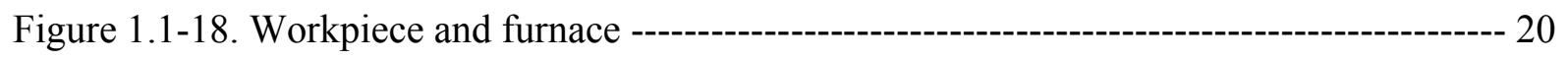

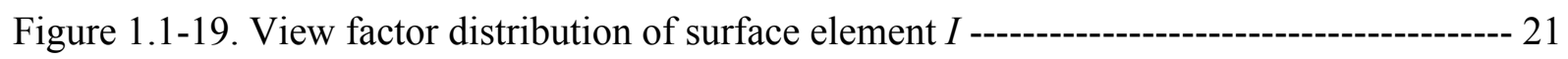

Figure 1.1-20. Temperature distribution during heating process ------------------------------------ 21

Figure 1.1-21. Comparison of calculated and measured temperature results of workpiece ------ 22 


\section{Lists of Figures, Tables and Appendices}

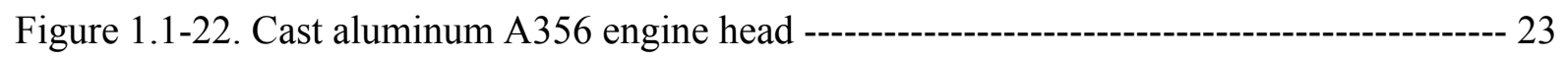

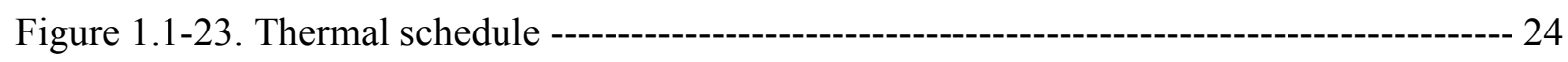

Figure 1.1-24. Predicted temperature profile of the load of engine heads (Unit: ${ }^{\circ} \mathrm{C}$ ) ------------- 24

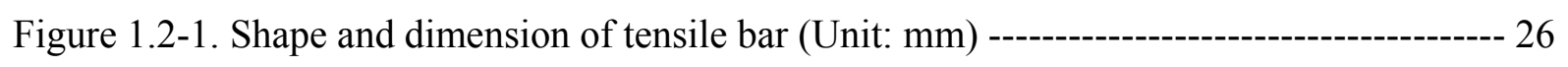

Figure 1.2-2. Two different load patterns ----------------------------------------------------------------- 27

Figure 1.2-3. Temperature profiles for two load arrangements. The four curves in each diagram from top to bottom represent thermal cycle, furnace temperature, temperature of the fastest heated point and the most slowly heated point of the load --------------------------------- 28

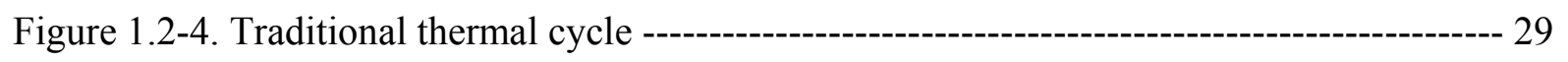

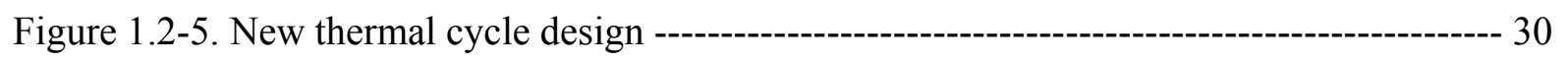

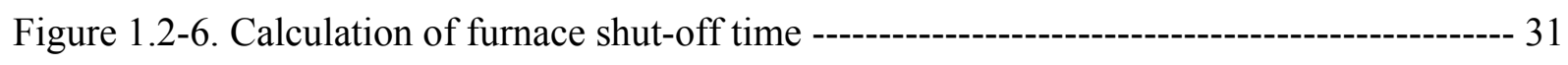

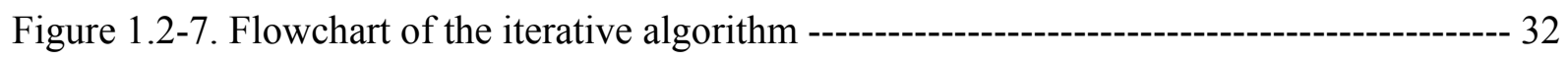

Figure 1.2-8. Temperature profile using the new thermal recipe. The four curves in each diagram from top to bottom represent thermal cycle, furnace temperature, temperature of the fastest heated point and the most slowly heated point of the load --

Figure 1.2-9 Temperature profile using traditional thermal cycle. The four curves in each diagram from top to bottom represent thermal cycle, furnace temperature, temperature of the

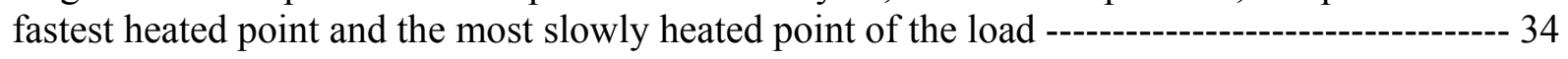

Figure 1.3-1. A cylindrical specimen of cast aluminum alloy A356 -------------------------------- 36

Figure 1.3-2. CHTE quench system ---------------------------------------------------------------------- 37

Figure 1.3-3. The variation of fluid velocity with the speed of impeller ---------------------------- 38

Figure 1.3-4. Cooling rate curves of cast aluminum alloy A356 quenched in different concentrations of polymer solution with medium level of agitation (1300rpm) ------------------ 40

Figure 1.3-5. Cooling rate curves of cast aluminum alloy A356 quenched in 20\% Aqua 260 at

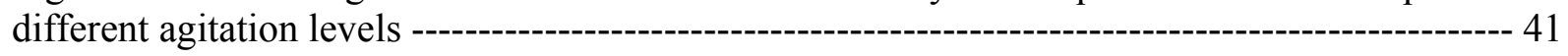




\section{Lists of Figures, Tables and Appendices}

Figure 1.3-6. Variations of average cooling rate of cast aluminum alloy A356 with polymer concentration and tank agitation

Figure 1.3-7. Contour plot of velocity field simulated in Fluent for case (a) 10\% Aqua 260 (b) $30 \%$ Aqua 260 upon 1300rpm agitation 46

Figure 1.3-8. Contour plot of velocity field in $20 \%$ Aqua 260 polymer solution simulated in Fluent for an agitation level of (a) 730rpm (b) 1950rpm 47

Figure 1.3-9. Vickers hardness of cast aluminum A356 solutionized, quenched in water, polymer solution and air, and aged at $165^{\circ} \mathrm{C}$ for different periods of times

Figure 1.3-10. Schematic illustrations on plot of $\mathrm{C}_{\mathrm{T}}$ function to calculate the quench factor ---- 51

Figure 1.3-11. Overview of the research methodology for quench factor analysis ---------------- 55

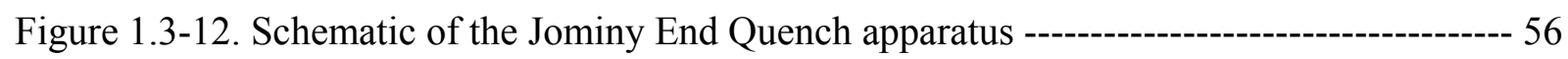

Figure 1.3-13. Microstructure of (a) as-cast and (b) as-solutionized cast aluminum A356 ------ 57

Figure 1.3-14. Measurement of SDAS of as-solutionized cast aluminum A356 ------------------ 58

Figure 1.3-15. Hardness profile of a Jominy End Quench bar of cast aluminum alloy A356 with different solutionizing times

Figure 1.3-16. Hardness profile of a Jominy End Quench bar of cast aluminum alloy A356 with different aging times

Figure 1.3-17. Cooling curves (a) and cooling rate curves (b) at different locations of a Jominy End Quench bar of cast aluminum alloy A356 63

Figure 1.3-18. Meyer hardness along a Jominy End Quench bar of cast aluminum A356 64

Figure 1.3-19. An example best fit curve for Quench Factor analysis of cast aluminum alloy A356 (0.5\% precipitation) $-66$

Figure 1.3-20. Time-Temperature-Property (TTP) curves for cast aluminum alloy A356 67

Figure 1.3-21. Racking pattern of cast aluminum A356 engine heads in a continuous furnace - 68

Figure 1.3-22. Cast aluminum A356 engine head instrumented with K-type thermocouples --- 68 


\section{Lists of Figures, Tables and Appendices}

Figure 2.0-1. Overall flow diagram for routines to simulate evolution of microstructure and solute distribution during solidification, post-solidification cooling, reheating, and solution heat treatment for aluminum-base casting alloys with silicon, copper, and/or magnesium ------------ 71

Figure 2.1-1. Binary phase diagram between elements A and B to illustrate phase equilibria data represented by module PHASECALC ---

Figure 2.1-2. Representation of curved liquidus and solidus boundaries by four straight-line segments, each. The phase boundaries are solid light gray curves and the simulated phase boundaries are the bold black line segments 84

Figure 2.1-3. Isothermal section through the A-B-C ternary alloy system at a temperature above the melting point of $\mathrm{C}$ and below the melting points of $\mathrm{A}$ and $\mathrm{B}$

Figure 2.1-4. Projection of several liquidus isotherms (solid lines) on a basal plane. Dotted triangles are flat triangular planes to represent the curved liquidus surface. The three corners of each triangular plane are labeled $\mathrm{i}, \mathrm{j}$, and $\mathrm{k}$

Figure 2.1-5. Storage scheme for tie line phase equilibrium data for the two-phase $\mathrm{L}+\alpha$ region in a quaternary system. The lower file represents storage of the temperature and composition end points for the $\alpha$-liquidus. The upper file represents storage of temperature and composition end points on the $\alpha$-solidus 86

Figure 2.1-6. Storage scheme for tie triangles phase equilibrium data for the three-phase $\mathrm{L}+\alpha+$ $\beta$. region in a quaternary system. The upper file represents storage of the temperature and composition end points for the $\alpha$-phase, the center file for the liquid, and the lower file for the $\beta$-phase 87

Figure 2.3-1. Al-6.5\% Si-3.5\% Cu, cast near a chill. White matrix is $\alpha$, dark gray particles are silicon, and light gray particles are $\theta$-phase. (Unetched, $350 \mathrm{X}$.) 113

Figure 2.3-2. Top: An array of dendrites of Al-Si-Cu alloy showing the position of the 1-D volume element. Bottom: The volume element showing a dendrite core of $\alpha$-phase and $\alpha$-phase + silicon-phase growing into the interdendritic liquid 114

Figure 2.3-3. Solidification curve for Al-6.5\% $\mathrm{Si}-3.5 \% \mathrm{Cu}$ for the Gulliver-Scheil (G-S) model 114

Figure 2.3-4. Distribution of $\mathrm{Si}$ and $\mathrm{Cu}$ in the $\alpha$-phase across the dendrite volume element Gulliver Scheil model 


\section{Lists of Figures, Tables and Appendices}

Figure 2.3-5. Distribution of $\mathrm{Si}$ and $\mathrm{Cu}$ in the $\alpha$-phase across the dendrite volume element limited solid diffusion model 115

Figure 2.3-6. Comparison of $\mathrm{Cu}$ distribution in the $\alpha$-phase for the Gulliver-Scheil (G-S) and

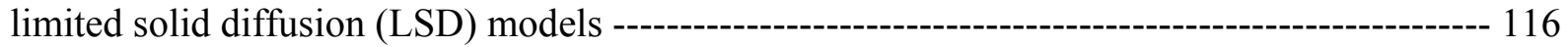

Figure 2.3-7. Comparison of Cu-distribution (top) and Si-distribution (bottom) in the $\alpha$-phase after cooling below the ternary eutectic to $573 \mathrm{~K}$, assuming no nucleation of silicon or $\theta$-phase particles from the $\alpha$-matrix (infinite nucleation barrier) vs. no undercooling before nucleation of precipitates from the $\alpha$-matrix (no nucleation barrier) 117

Figure 2.3-8. Comparison of $\mathrm{Cu}$ distribution in the $\alpha$-phase for the enhanced diffusion and limited solid diffusion models 118

Figure 2.3-9. Comparison of $\mathrm{Cu}$ distribution in the $\alpha$-phase for the limited solid diffusion and divorced eutectic models 118

Figure 2.3-10. Extended models for solidification and solution treatment simulation

Figure 2.3-11. Comparison of the $\mathrm{Cu}$ (upper) and $\mathrm{Si}$ (lower) distribution after solidification and cooling to $300^{\circ} \mathrm{C}$ for an $\mathrm{Al}-7 \% \mathrm{Si}-3.5 \% \mathrm{Cu}$ alloy predicted by the original LSD model and the divorced eutectic model 120

Figure 2.3-12. $\mathrm{Cu}$ distribution in $\alpha$-phase for $\mathrm{Al}-7 \% \mathrm{Si}-3,5 \% \mathrm{Cu}-0.5 \% \mathrm{Mg}$ alloy after solidification to the quaternary eutectic temperature: Comparison of use of $\mathrm{D}_{\mathrm{CuCu}}$ (LSD) measured for ternary alloy without $\mathrm{Mg}$ and use of enhanced diffusivity $\left(2 \mathrm{xD}_{\mathrm{CuCu}}\right)$ for $\mathrm{Mg}$ addition (ESD-2)

Figure 2.3-13. Comparison of predicted $\mathrm{Cu}$ distribution in $\alpha$-phase for as-cast Al-7\% $\%$ i-3.5\%Cu-0.5\%Mg with microprobe data (points). Model ESD-2 includes divorced solidification, enhanced diffusivity due to $\mathrm{Mg}$ addition and enhanced diffusion in $\alpha+$ silicon region 121

Figure 2.4-1. Relative rate of dissolution of $\mathrm{CuAl}_{2}$ at three solution treatment temperatures and for three as-cast dendrite arm spacings in $\mathrm{Al}-6.5 \% \mathrm{Si}-3.5 \% \mathrm{Cu}$ alloy 132

Figure 2.4-2. Rate of dissolution of $\theta$-phase in Al-6.5\% $\mathrm{Si}-3.5 \% \mathrm{Cu}$ alloy plotted for three solution treatment temperatures as a function of solution treatment time divided by half of the dendrite arm spacing squared 133

Figure 2.4-3. Dissolution of $\theta$-phase during solution treatment of $\mathrm{Al}-7 \% \mathrm{Si}-3.5 \% \mathrm{Cu}$ at $505^{\mathrm{O}} \mathrm{C}$ : Comparison of 1-D and 2-D models 133 


\section{Lists of Figures, Tables and Appendices}

Figure 2.4-4. Copper (top) and magnesium (bottom) distributions in the $\alpha$-phase for a quaternary $\mathrm{Al}-7 \% \mathrm{Si}-3.5 \% \mathrm{Cu}-0.5 \% \mathrm{Mg}$ alloy after several solution treatment times at $505^{\mathrm{O}} \mathrm{C}$ 134

Figure 2.4-5. Rate of dissolution of Q-phase (top) and $\theta$-phase (bottom) for quaternary alloy solution treated at $480^{\circ} \mathrm{C}$ 135

Figure 3.1-1. Material database structure 137

Figure 3.1-2. Material database management 138

Figure 3.1-3. Material type management 138

Figure 3.1-4. Material name management 138

Figure 3.1-5. Material thermal properties management 139

Figure 3.1-6. Atmosphere database management 139

Figure 3.1-7. Fuel database management 141

Figure 3.2-1. Furnace database management 141

Figure 3.4-1. Equilibrium tie line data for a quaternary alloy system stored for access by PHASECALC. Order of phase appearance on cooling the liquid is $\alpha$-phase aluminum rich terminal solid solution, silicon, $\mathrm{Mg}$ and $\mathrm{Cu}$ rich Q-phase, and $\mathrm{CuAl}, \theta$-phase 148

Figure 3.4-2. Microstructure of cast and heat-treated alloy 319 from an automotive component. The color etch turns the silicon particles blue and the iron-rich intermetallic brown 149

Figure 3.4-3. SEM images and X-ray spectra semi-quantitative analyses of constituent particles in Al-319 automotive castings 150

Figure 3.4-4. SEM image of metallographic section cut from end-chilled plate casting. Phases containing low atomic weight elements ( $\mathrm{Al}, \mathrm{Si}, \mathrm{Mg}$ ) appear dark and phases containing heavy elements $(\mathrm{Cu}, \mathrm{Fe})$ appear bright. Iron-rich needles are $\mathrm{Al}_{5} \mathrm{FeSi}$. Interdendritic $\theta$-phase $\left(\mathrm{CuAl}_{2}\right)$ and silicon are evident 151

Figure 3.4-5. Illustration of grid of measurement points used for electron beam microprobe analysis of solute distribution averaged over a representative number of dendrite arms --------152

Figure 3.4-6. Example of averaged copper distribution across a characteristic dendrite volume element obtained by electron beam microprobe analysis 153 


\section{Lists of Figures, Tables and Appendices}

Figure 3.4-7. Cooling curve for an $\mathrm{Al}-7 \% \mathrm{Si}-3.5 \% \mathrm{Cu}-0.5 \% \mathrm{Mg}$ quaternary alloy and (at bottom) the time derivative of the cooling curve. Thermal arrests and/or recalescence on the cooling curve and peaks/dips in the derivative curve mark temperatures for the initiation of phase formation 154

Figure 3.4-8a. Interrupted solidification samples. Left: Sample quenched at $571^{\circ} \mathrm{C}$ shows formation of $\alpha$-phase dendrites. Right: Sample quenched at $564^{\mathrm{O}} \mathrm{C}$ shows continued formation of $\alpha$-phase dendrites and initiation of iron-rich intermetallic, $\mathrm{Al}_{5} \mathrm{SiFe}$ 155

Figure 3.4-8b. Interrupted solidification samples. Left: Sample quenched at $571^{\circ} \mathrm{C}$ shows formation of $\alpha$-phase dendrites. Right: Sample quenched at $564^{\mathrm{O}} \mathrm{C}$ shows continued formation of $\alpha$-phase dendrites and initiation of iron-rich intermetallic, $\mathrm{Al}_{5} \mathrm{SiFe}$ 156

Figure 3.4-8c. Interrupted solidification samples. Left: Sample quenched at $545^{\circ} \mathrm{C}$ shows continued formation of ${ }^{\sim}$ dendrites and $\mathrm{Al}_{5} \mathrm{FeSi}$ platelets and initiation of $\alpha+$ silicon colonies. Some $\mathrm{Al}_{5} \mathrm{FeSi}$ platelets are converting to $\mathrm{Cu}_{2} \mathrm{FeAl}_{7}$. Right: Same $-157$

Figure 3.4-8d. Interrupted solidification samples. Left: Sample quenched at $520^{\circ} \mathrm{C}$ shows continued formation of $\alpha$-dendrites, $\mathrm{Al}_{5} \mathrm{FeSi}$ platelets, $\alpha+$ silicon colonies, and conversion of $\mathrm{Al}_{5} \mathrm{FeSi}$ to $\mathrm{Cu}_{2} \mathrm{FeAl}_{7}$. Right: Sample quenched at $510^{\circ} \mathrm{C}$. $\mathrm{Al}_{2} \mathrm{Cu}, \theta$-phase, has formed at quaternary eutectic 158

Figure 3.6-1. Schematic diagram of test plate casting showing placement of copper end chill and cylindrical riser $-173$

Figure 3.6-2. Tensile strength, yield strength $(0.1 \%$ offset $), \&$ plastic strain $(\%)$ vs. solution treatment time $\&$ dendrite arm spacing, nominal alloy 174

Figure 3.6-3. Variation in dendrite arm spacing and volume percent porosity with distance from the chill in end-chilled plate castings 175

Figure 3.6-4. Comparison of measured tensile strength (points) and computed tensile strength (curve) vs. solution treatment time 175

Figure 3.6-5. Comparison of measured tensile strength and computed tensile strength -------- 176

Figure 3.6-6. Validation: Comparison of measured tensile strength and predicted tensile strength for additional cast plates 176

Figure 3.6-7. Comparison of measured tensile strength and isostrength lines computed with the empirical relation for as-cast dendrite arm spacing and volume percent porosity 177 


\section{Lists of Figures, Tables and Appendices}

Figure 3.6-8. UTS vs distance from the chill and solution treatment time for alloys with low (top) and high (bottom) copper in comparison to the nominal composition 178

Figure 3.6-9. \% elongation (plastic) vs distance from the chill and solution treatment time for alloys with low (top) and high (bottom) $\mathrm{Cu}$ in comparison to the nominal composition ------- 179

Figure 3.6-10. UTS vs distance from the chill and solution treatment time for alloys with low (top) and high (bottom) $\mathrm{Si}$ in comparison to the nominal composition 180

Figure 3.6-11. \% elongation (plastic) vs distance from the chill and solution treatment time for alloys with low (top) and high (bottom) Si in comparison to the nominal composition -------- 181

Figure 3.6-12. UTS vs distance from the chill and solution treatment time for alloys with low Fe (top) and low Mg (bottom) in comparison to the nominal composition ---------- 182

Figure 3.6-13. \%Elongation (plastic) vs distance from the chill and solution treatment time for alloys with low $\mathrm{Fe}$ (top) and low $\mathrm{Mg}$ (bottom) in comparison to the nominal composition ---- 183

Figure 3.6-14. Tensile strength, yield strength ( $0.2 \%$ offset-dashed line), and plastic strain (\%) vs. solution treatment time (hrs) for dendrite arm spacings: $17 \mu \mathrm{m}$ (squares), $35 \mu \mathrm{m}$ (diamonds), 44 $\mu \mathrm{m}$ (triangles), $61 \mu \mathrm{m}$ (circles) $-184$

Figure 3.6-15. Comparison of computed and measured tensile strengths for nominal Al-Si-Mg

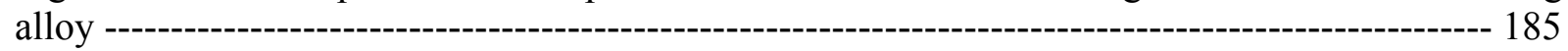

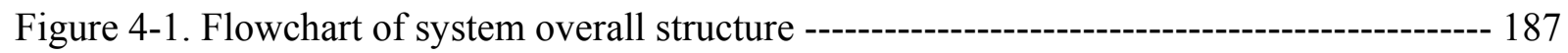

Figure 4.2-1. Flowchart of microstructure evolution simulation ----------------------------- 189

Figure 4.2-2. Relationship between main module and sub-module -------------------------------- 190

Figure 4.2-3. Relationship between sub-module and database ------------------------------------- 191

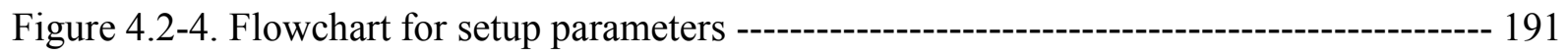

Figure 4.3-1. Correlation of measured tensile strength and the calculated one using given

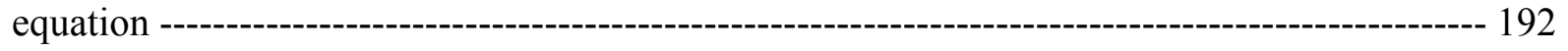

Figure 4.4-1. Workpiece shape and dimension (Unit: mm) ----------------------------------------- 193

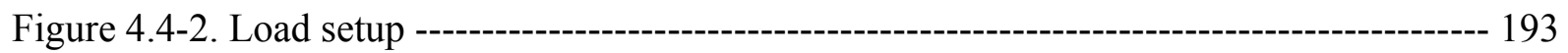




\section{Lists of Figures, Tables and Appendices}

Figure 4.4-3. Relative position of furnace and load ---------------------------------------------- 193

Figure 4.4-4. Positions of thermal couples --- 193

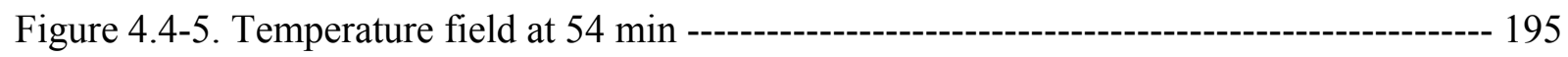

Figure 4.4-6. Comparison between experimental and calculated temperature profiles --------- 196

Figure 4.4-7. Variation of weight fraction of $\theta$ phase during solution treatment ----------------- 197

Figure 4.4-8. Variation of weight fraction of Q phase during solution treatment ----------------- 197

Figure 4.4-9. Variation of tensile strength during solutionizing -------------------------------------- 198

Figure 4.4-10. Variation of yield strength during solutionizing ---------------------------------------198

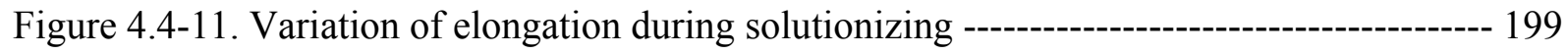




\section{Lists of Figures, Tables and Appendices}

Table 1.1-1. Comparison of finite difference method and finite element method ------------------ 4

Table 1.2-1. Thermal cycle variables and levels fore the simulation ---------------------------------- 27

Table 1.2-2. Performance comparison between two load arrangements ---------------------------- 29

Table 1.2-3 Input data for thermal recipe optimization ------------------------------------------------ 32

Table 1.2-4. Comparison between the traditional and new thermal cycles ------------------------- 34

Table 1.3-1. Chemical composition of cast aluminum alloy A356 (wt\%) ------------------------ 36

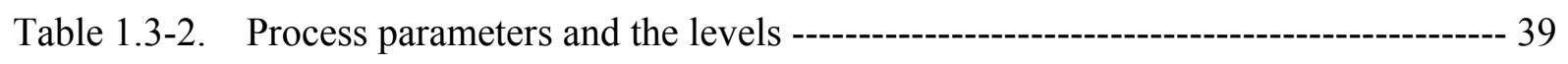

Table 1.3-3. Taguchi L9 Layout (Three-Level orthogonal arrays) ---------------------------------- 39

Table 1.3-4. ANOVA analysis table for average cooling rate ----------------------------------------- 43

Table 1.3-5. Parameters used in CFD simulation ------------------------------------------------------ 44

Table 1.3-6. Velocity magnitude from the Fluent simulation ---------------------------------------- 45

Table 1.3-7. Simulated and measured velocity at one end of the U-shaped tube ------------------ 47

Table 1.3-8. Test matrix for studying the aging kinetics of cast aluminum A356 ----------------- 49

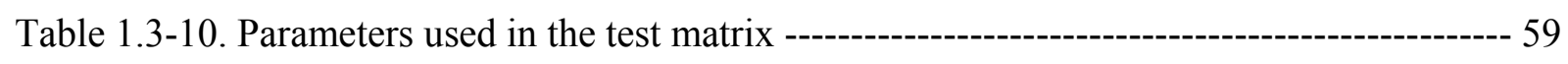

Table 1.3-11. Distance from the quench end where experimental data were collected ----------- 61

Table 1.3-12. Precipitation kinetic parameters of cast aluminum alloy A356 during quenching 66

Table 1.3-13. Predicted and measured hardness of a cast A356 engine head ----------------------- 69

Table 3.5-1. Diffusion Coefficients of Copper in Al-Si-Cu-Mg Alloys --------------------------- 159

Table 3.5-2. Diffusion Coefficients of Silicon in Al-Si-Cu-Mg Alloys --------------------------- 160

Table 3.5-3. Diffusion Coefficients of Copper in Single Phase $\alpha$-Al and two phase $\alpha$-Al +13 vol.\% Si alloys 160 


\section{Lists of Figures, Tables and Appendices}

Table 3.6-1. Aim Compositions and Melt Treatments for Test Plate Castings ---------------- 172

Table 4.4-1. Thermal schedule 195 


\section{Executive Summary}

The model development and verification specified in this project have been successfully completed. An overview of each module and its verification is presented in the Executive Summary. The details of each module are presented in the body of the report.

\section{Thermal prediction and optimization}

The thermal prediction modules, HTFURNACE and QUENCHCALC, were created to model the temperature distributions within the furnace and load during heating and quenching processes. The temperature of an aluminum alloy part is determined as a function of time and position during heating and quenching. Modules PTLOAD and TPCONTROL are built for load pattern optimization and furnace control.

Both of HTFURNACE and QUENCHCALC are based the same numerical method-Finite Difference Method (FDM). HTFURNACE has been developed with the entire furnace system model, including the load, furnace walls, heating elements and cooling water/air. The heat transfer calculations include conduction, radiation with nonparticipating media and empirical convection. The model is integrated with CAD software with pre- and post-processing for FDM. In QUENCHCALC, the quenching conditions include the heating temperature distribution in the part from HTFURNACE as the initial condition as well as the effective surface heat transfer coefficients for the alloy as a function of quenching fluid temperature, part surface temperature, surface condition, part geometry (i.e. features) and part orientation as the boundary condition. The temperature versus time calculations at selected locations in the part are used to predict the microstructure and/or mechanical properties of the part at the selected locations by solutionizing modules.

PTLOAD was developed to quantitatively evaluate the process performance for different designs, i.e., load arrangement designs. The thermal profile of each design is achieved through simulation. A relative cost function on a per-unit-load-mass basis is then used to determine the load xiii 


\section{Executive Summary}

arrangement by choosing the one which minimizes relative cost function by considering four aspects: a) "Heat-up Time" is defined as the length of time during which the most slowly heated point of the load is heated from room temperature to the soaking temperature; b) "Energy Consumption" is defined as the total energy input to the furnace-load system from the start of heating to the time when the entire load reaches soaking temperature; c) "Temperature Nonuniformity" is defined as the maximum temperature difference that has ever appeared within the load during heat-up period; d) "Temperature Nonuniformity" is a representative of the loss of overall quality or equivalent increase of cost. This is because the large temperature difference within the load will lead to higher risk of thermal stress or even crack in parts.

TPCONTROL is to optimize thermal schedule to increase heating rates. Original thermal cycle is re-designed that the furnace is first heated to and kept at an elevated temperature above soaking temperature, and after a "proper" length of time, the furnace is shut off, and it releases heat until the furnace temperature decreases to the soaking temperature. The load absorbs heat and the load temperature will keep increasing. The longer the furnace is kept at the elevated temperature before shut-off, the faster the load temperature rises. Therefore TPCONTROL is to determine the elevated temperature and shut-off time.

\section{Solutionizing prediction}

Solutionizing prediction modules, PHASECALC, DIFFCALC, CASTSEG and CASTSOLN were developed to simulate and predict the evolution of microstructure of cast aluminum alloys during a specified thermal cycle.

The major function of PHASECALC is to represent phase equilibria in multicomponent alloys in the temperature ranges relevant to solidification and solution heat treatment. The phase equilibria representation procedures are based on the shape (interpolation) functions used in finite element analyses. We are extending procedures used to represent phase equilibria for binary (e.g., Al-Cu and Al-Si alloys) and ternary alloys (e.g., Al-Si-Cu alloys) to more complex alloy systems by writing algorithms in general terms that can be applied to even higher order systems. 


\section{Executive Summary}

DIFFCALC is designed to calculate diffusion coefficients that are needed to simulate the kinetic behavior of multicomponent industrial alloys during casting and heat treatment.

CASTSEG simulates solute distribution during solidification to obtain the initial condition for heat treatment that is representative of the specific casting parameters. CASTSEG is for binary and ternary systems, including $\mathrm{Al}-\mathrm{Si}, \mathrm{Al}-\mathrm{Cu}$, and $\mathrm{Al}-\mathrm{Si}-\mathrm{Cu}$. The module is used for binary eutectic and ternary eutectic alloy systems. It is being used iteratively with experiment to validate and improve the underlying models and to gather kinetic and thermodynamic data. CASTSOLN is to handle alloy systems with more than three components.

The major objective of the module CASTSOLN is to quantitatively describe the evolution of as-cast microstructure (phase amounts, morphologies, and microsegregation) during post-solidification thermal cycles including, in particular, solution heat treatment of industrial multi-component alloys. It takes the output of PHASECALC, DIFFCALC and CASTSEG as input. It has been developed and used for $\mathrm{Al}-6.5 \% \mathrm{Si}-3.5 \% \mathrm{Cu}$.

\section{Databases}

The databases were being developed by mining the existing literature and by selected experimentations. The data mined from literatures are compared with each other and analyzed, and some are also compared with the experimental results.

The databases in the project are classified into following databases:

- Heat transfer databases:

o Material database;

o Atmosphere database;

o Fuel/gas database;

- Quenching performance database;

- Furnace database;

- Phase diagram for a selected alloy system (Al-Si-Cu);,

- Diffusivity versus composition and temperature;, 


\section{Executive Summary}

- Properties versus porosity, si particle size, undissolved $\theta$ and grain size.

\section{Integration of Thermal-Microstructure-Property Prediction}

Finally, all the modules were integrated together to build a system of software, databases, and design rules to enable quantitative prediction and optimization of the heat treatment of aluminum castings to increase quality, increase productivity, reduce heat treatment cycle times and reduce energy consumption. The integrated model consists of three functionally independent while data-wise correlated modules: (a) thermal prediction; (b) alloy microstructure evolution prediction; and (c) mechanical property prediction. The thermal prediction was implemented first. The simulation results are taken together with the alloy's as-cast conditions as input to the other two modules for predictions of microstructure and mechanical properties. The software can predict the thermal cycle in critical locations of individual components in a furnace, the evolution of microstructure, and the attainment of properties in heat treatable aluminum alloy castings. The system offers a quantitative understanding of the kinetics of microstructure evolution in complex multicomponent alloys, on a quantitative understanding of the interdependence of microstructure and properties, on validated kinetic and thermodynamic databases, and validated quantitative models.

\section{Commercialization}

The commercialization plan is proceeding with continuing discussions with the ESI Group, a world leading organization for numerical simulation of manufacturing processes including ProCAST (casting simulation software). The addition of the models developed and verified in this project fit very well into their current portfolio of software products. 


\section{Introduction}

The objective is to develop, verify and market an integrated system of software, databases, and design rules to enable quantitative prediction and optimization of the heat treatment of aluminum castings to increase quality, increase productivity, reduce heat treatment cycle times and reduce energy consumption. The software will predict the thermal cycle in critical locations of individual components in a furnace, the evolution of microstructure, and the attainment of properties in heat treatable aluminum alloy castings. The model will take into account the prior casting process and the specific composition of the component. The heat treatment simulation modules will be used in conjunction with software packages for simulation of the casting process. The system will be built upon a quantitative understanding of the kinetics of microstructure evolution in complex multicomponent alloys, on a quantitative understanding of the interdependence of microstructure and properties, on validated kinetic and thermodynamic databases, and validated quantitative models.

One of the most important research needs identified as a top priority by industry leaders at strategic planning workshops sponsored by the Department of Energy Office of Industrial Technology (DOE-OIT) is the development of integrated process models, the topic of the current research and development program. The goal is to change heat treating from an experience-based art to a process that is truly understood and capable of being simulated.

The program is being managed under the auspices of the Center for Heat Treating Excellence (CHTE), a consortium of over fifty companies. The CHTE is a virtual center in that research is carried out at several universities and industrial sites, where the appropriate experience and facilities are located. Industry support and collaboration are being provided by an industry focus group that provides materials, testing, software, and specialized training. In addition they promote the transfer and commercialization of technology developed under this program in their companies.

The plan is that the industry-university team will develop, verify and integrate their process modules for the heat treatment of aluminum castings. Then the modules will be transferred to industry to be further integrated with commercial casting simulation and casting design software 


\section{Introduction}

packages. Databases for the thermophysical and kinetic properties that enable the quantitative prediction of the evolution of microstructure and the attainment of specified strength, ductility, and fatigue properties in critical locations of aluminum castings will be developed from the literature and where necessary validated and extended by experiment. The methodology applied to the development of a model for aluminum castings can serve later as a framework to develop quantitative process models for other alloy systems, including ferrous alloys. 


\section{Section 1: Thermal Prediction}

Thermal Prediction Modules consist HTFURNACE and QUENCHCALC modules. Software has being developed to model and simulate the heat transfer and temperature distribution of parts during the heating process in a furnace and the quenching process. Optimization modules PTLOAD and TPCONTROL are built for load pattern optimization and furnace control. The functions and development of the major modules are listed below.

\subsection{HTFURNACE:}

The HTFURNACE module aims at prediction of temperature distribution and the thermal history of specified spots in aluminum casting during heating process. It provides temperature distribution for quenching and thermal history of specified spots for microstructure prediction.

\subsubsection{Modeling methodology}

In numerical simulation there are two popular methods: Finite Difference Method (FDM) and Finite Element Method (FEM). The comparison of these two methods is listed in Table 1.1-1. FEM is more complicated. The size and shape of its elements at different locations vary with the local shape and dimension of the CAD model. Because of the variation of elements' shape and size in FEM, its pre-and post- processor is also much more complicated than FDM. FDM is not as complicated as FEM. Its elements are blocks which are controlled by length, width and height. It is easier for industrial application and software development. The calculation precision can be guaranteed by much more finer elements of the calculation domain. Therefore, FDM is selected as the numerical method in this project. 


\section{Section 1: Thermal Prediction}

Table 1.1-1. Comparison of finite difference method and finite element method

\begin{tabular}{|l|l|l|}
\hline Element & FDM & FEM \\
\hline Popular used fields & $\begin{array}{l}\text { Element sizes are strictly } \\
\text { controlled by the steps in } \mathrm{x}, \mathrm{y}, \\
\mathrm{z} \text { directions. } \\
\text { Regular shape: rectangular for } \\
\text { 2-D, cubic for 3-D }\end{array}$ & $\begin{array}{l}\text { Element sizes are just roughly } \\
\text { controlled. } \\
\text { Irregular shape: more shapes } \\
\text { like tetrahedral, hexahedral }\end{array}$ \\
\hline Enmeshment & $\begin{array}{l}\text { Heat transfer, fluid flow, } \\
\text { microstructure prediction }\end{array}$ & Mechanics \\
\hline Variables' location & Simple, automatically & $\begin{array}{l}\text { Very complicated. Manual and } \\
\text { automatically. But sometimes } \\
\text { the interference is necessary. }\end{array}$ \\
\hline Calculation methods & At the center of each element & $\begin{array}{l}\text { At the corner, edge center and } \\
\text { geometrical center. }\end{array}$ \\
\hline Pre- and post- processing & Explicit and implicit & $\begin{array}{l}\text { Decomposition of triangle } \\
\text { matrix and iteration }\end{array}$ \\
\hline
\end{tabular}

In this project, the technical route shown in Figure 1.1-1, which includes construction of 3-D geometrical models, finite difference enmeshment, heat transfer models (including conduction, convection and flow, and radiation), PID control and pre- and post-processors. 


\section{Section 1: Thermal Prediction}

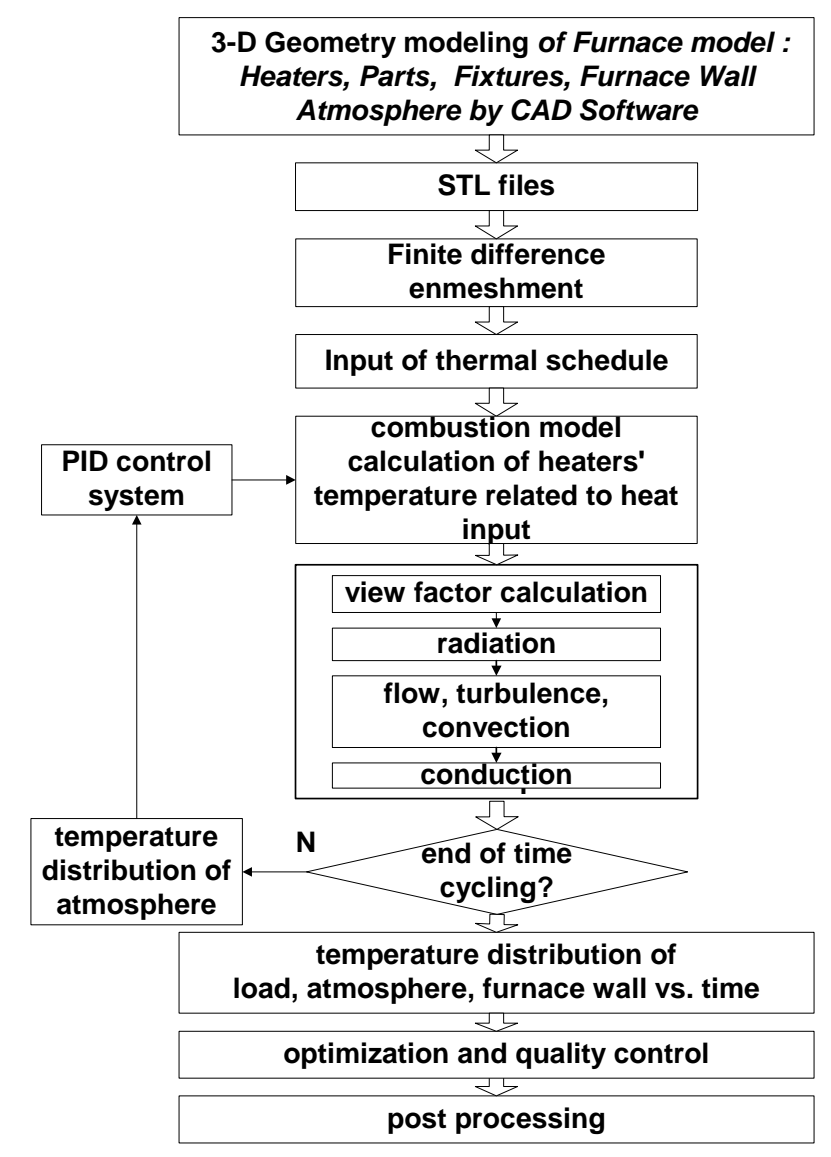

Figure 1.1-1. The technical route of modeling of heat transfer in heat treatment furnace

\subsubsection{Heat transfer models}

The heat transfer in heat treatment furnaces is controlled by conduction, convection, radiation and furnace control. Conduction occurs in all solid materials. Convection exists between the atmosphere and solid materials exposed to it, and furnace walls to the ambient air. Radiation exists between seperated solid materials. Furnace control refers to the PID control (proportional, integral and derivative). Finite difference method (FDM) is used to simulate the heat transfer in the heat treatment furnace system. The enmeshed furnace system is shown in Figure 1.1-2. Workpieces, furnace wall, heaters, cooling water, cooling air, fixture and etc. are included in the furnace system. 


\section{Section 1: Thermal Prediction}

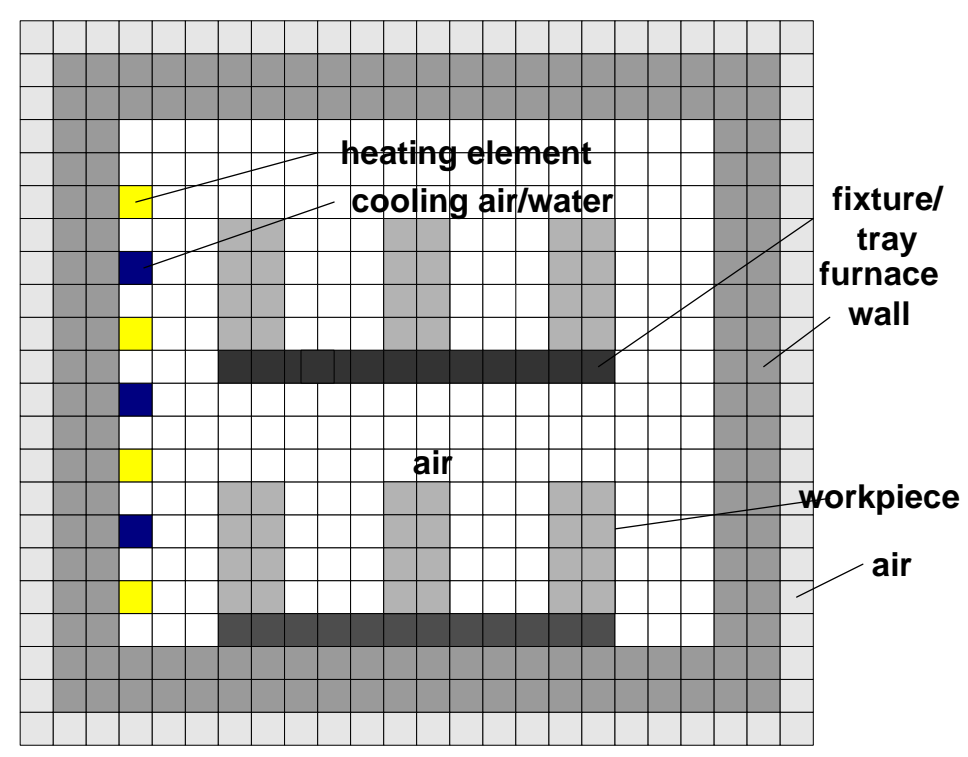

Figure 1.1-2. Enmeshed furnace model

\section{1) Conduction model}

The differential equation for conduction is as follows[1]:

$$
\rho \cdot c \frac{\partial T}{\partial t}=\lambda\left(\frac{\partial^{2} T}{\partial x^{2}}+\frac{\partial^{2} T}{\partial y^{2}}+\frac{\partial^{2} T}{\partial z^{2}}\right)+q_{v}
$$

where $\rho$ is the density, $c$ is the specific heat, $\lambda$ is the thermal conductivity, $T$ is temperature, $t$ is time, $q_{\mathrm{v}}$ is the energy source term.

Then it is discretized as follows,

$$
\begin{aligned}
T_{i, j, k}^{m+1} & =T_{i, j, k}^{m}+\frac{\lambda \cdot \Delta t}{\rho \cdot c}\left(\frac{T_{i+1, j, k}^{m}-2 T_{i, j, k}^{m}+T_{i-1, j, k}^{m}}{\Delta x^{2}}+\frac{T_{i, j+1, k}^{m}-2 T_{i, j, k}^{m}+T_{i, j-1, k}^{m}}{\Delta y^{2}}\right. \\
& \left.+\frac{T_{i, j, k+1}^{m}-2 T_{i, j, k}^{m}+T_{i, j, k-1}^{m}}{\Delta z^{2}}\right)+\frac{\Delta t}{\rho \cdot c} \cdot q_{v}
\end{aligned}
$$




\section{Section 1: Thermal Prediction}

where $m$ is the time step.

The thermal conductivity is calculated using equation (1.1-3) for the conduction between two different solid materials (Figure 1.1-3).

$$
\lambda_{i_{-} i+1}=\frac{1}{R_{i_{-} i+1}}=\frac{1}{\frac{\frac{\Delta x_{i, j, k}}{2}}{\lambda_{i, j, k}}+\frac{\frac{\Delta x_{i+1, j, k}}{2}}{\lambda_{i+1, j, k}}}
$$

where $R$ is the thermal resistance.

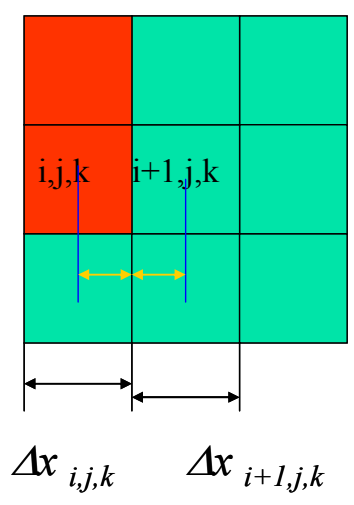

Figure 1.1-3. Thermal resistance between different type materials

\section{1) Boundary conditions}

\section{- Solid surface}

For the solid surface usually there are convection and radiation, for example, the furnace wall external surface and internal surface, casting surface. For vacuum furnace, there only exists radiation between solid surfaces inside the furnace. Then on the solid surface, the boundary heat flux is expressed as 


\section{Section 1: Thermal Prediction}

$$
Q=Q_{\text {rad }}+Q_{\text {conv }}
$$

Boundary radiation $Q_{\text {rad }}$ and convection $Q_{\text {conv }}$ flux will be explained in the other sections.

\section{- Heating elements}

Except the convection and radiation, the boundary conditions of heating elements also include the heat input which is decided by PID control and furnace power.

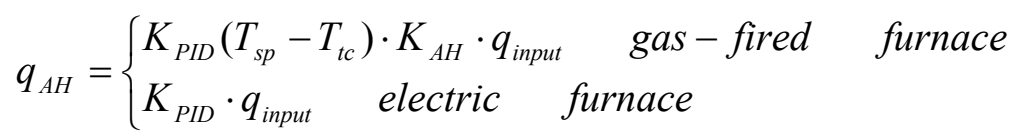

Where $K_{P I D}$ is the constant determined by PID control (refers to section 1.1.3), $K_{A H}$ is the constant determined by combustion for gas-fired furnace.

\section{2) Radiation model}

\section{- Assumptions}

The radiation models are presented based on the following assumptions:

a) Nonparticipating atmosphere or no atmosphere;

b) Atmosphere temperature is uniform.

The radiation calculation is by

$$
Q_{\text {rad }}=\sum_{p^{\prime}=1}^{N} \sigma \cdot \varepsilon \cdot A_{p} \cdot F v_{p p^{\prime}}\left(T_{p}{ }^{4}-T_{p^{\prime}}{ }^{4}\right)
$$

where $\sigma$ is the Stefan-Boltzmann constant, $\varepsilon$ is the emissivity, $F v$ is the view factor, $A$ is the surface area. 


\section{Section 1: Thermal Prediction}

\section{- View factor calculation}

Radiation occurs inside every element surface pairs. The calculation volume is very huge. Therefore the view factor calculation is simplified, as shown in Figure 1.1-4. For a source surface, two types of radiation target surfaces exist in the finite difference model, one is the surface perpendicular to the source surface, the other one is that parallel to it. The radiation between the source surface and the perpendicular surfaces is mainly limited to the four closely neighbor surfaces. For the surfaces parallel to the source surface the view factor will decrease rapidly from the right opposite surface to the outside surrounding surfaces. Then, to simplify the view factor calculation, the four surrounding perpendicular surfaces and $n^{*} n$ square parallel surfaces are considered. The calculation precision is adjustable by control of $n$. The view factor equation is equation (1.1-7).

$$
F v_{p p^{\prime}}=\frac{A_{p} A_{p^{\prime}} \cos \alpha \cdot \cos \beta}{\pi \cdot d^{2}}
$$

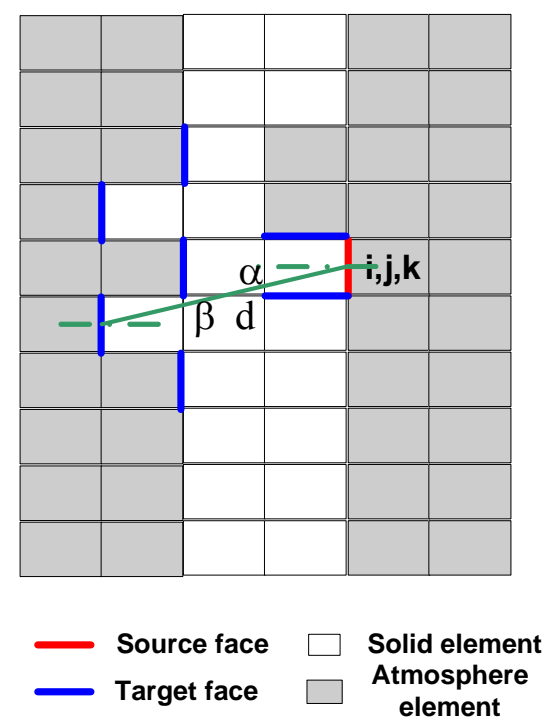

Figure 1.1-4. 2-D illustrative diagram of view factor calculation 


\section{Section 1: Thermal Prediction}

\section{3) Convection model}

There are two kinds of convection: natural and forced. For example the circulation fan does not work during heating process in vacuum furnace, thus there is natural convection presented. Under some conditions the fan is turned on, so forced convection occurs. The convection heat transfer between the furnace and the workpiece is denoted as follows:

$$
Q_{\text {conv }}=h_{\text {conv }} A_{i, j, k_{-} \text {conv }}\left(T_{i, j, k}-T_{g}\right)
$$

where $h_{\text {conv }}$ is the convection film coefficient, which can be calculated by the following equation:

$$
h_{c o n v}=\frac{\lambda_{g}}{L^{*}} \cdot N u_{L^{*}}
$$

where $L^{*}$ is the characteristic length, $L^{*}=A^{1 / 2} . \lambda_{g}$, is the thermal conductivity of gas, $N u_{L^{*}}$, the Nusselt number, is related to the geometrical features of workpiece, load pattern, thermal properties of atmosphere and circulation fan. The calculations of $N u_{L^{*}}$ are different for natural convection and forced convection.

\section{- Natural convection}

For natural convection the workpiece arrangement has no specific effect on the natural convection model of heat transfer. The Nusselt number is calculated by [1]

$$
N u_{L^{*}}=3.47+0.51 \cdot R a_{L^{*}}^{1 / 4} \quad(P r \geq 0.7)
$$

where $P r$ is the Prandtl number, $R a_{L^{*}}$ is the Rayleigh number, calculated by

$$
R a_{L^{*}}=\frac{g \cdot \beta \cdot\left(T_{\infty}-T_{w p}\right) \cdot L^{* 3}}{\alpha \cdot v} \quad\left(0<R a_{L^{*}}<10^{8}\right)
$$




\section{Section 1: Thermal Prediction}

where $v$ is kinetic viscosity, $\alpha$ is thermal diffusivity, $\beta$ is the coefficient of volumetric thermal expansion for an ideal gas, $T_{w p}$ is the load temperature, $T_{\infty}$ is the atmospheric temperature, and $g$ is the gravity coefficient.

The Prandtl number is determined using

$$
\operatorname{Pr}=v / \alpha
$$

The thermal diffusivity $\alpha$ is defined as follows.

$$
\alpha=\lambda_{g} /\left(\rho_{g} \cdot c_{g}\right)
$$

where $\rho_{g}$ and $c_{g}$ are the density and specific heat of gas, respectively.

\section{- Forced convection}

For forced convection heat transfer, methods for the calculations of the Nusselt number for single workpiece and for an array of workpieces are also different.

For a single workpiece, the Nusselt number $N u_{L^{*}}$ can be calculated using the following experimental equation[1]

$$
\begin{aligned}
N u_{L^{*}}=N u_{L^{*}}^{0}+ & {\left[0.15\left(\frac{p}{L^{*}}\right)^{1 / 2} \cdot \operatorname{Re}_{L^{*}}^{1 / 2}+0.35 \operatorname{Re}_{L^{*}}^{0.566}\right] \cdot \operatorname{Pr}^{1 / 3} } \\
& \left(0<R e_{L^{*}}<2 \times 10^{5}, \operatorname{Pr}>0.7,0<C / B<5\right)
\end{aligned}
$$

where $\mathrm{C} / \mathrm{B}$ is the ratio of axial lengths of the workpiece, $p$ is the maximum perimeter of the workpiece perpendicular to the flow direction; $N u_{L^{*}}^{0}$ can be found from reference tables. $R e_{L^{*}}$, Reynolds number, is denoted by 


\section{Section 1: Thermal Prediction}

$$
\operatorname{Re}_{L^{*}}=\frac{U_{\infty} \cdot L^{*}}{v}
$$

where $U_{\infty}$ is the flow speed of atmospheric gas,

$$
U_{\infty}=\frac{\frac{\pi \times D_{f a n}^{2}}{4.0} H_{f a n} \cdot R_{f a n}}{A_{w s}}
$$

where $D_{f a n}, H_{f a n}, R_{f a n}$ are the diameter, height and rotation speed of the circulation fan respectively, and $A_{w s}$ is the area of the load facing the atmospheric gas flow direction.

The calculations of $N u_{D^{*}}$, for aligned and staggered workpiece loads, as shown in Figure 1.1-5, can be found in the reference[1]. Here the equivalent diameter $D^{*}$ is the same as the equivalent length $L^{*}$. N is the number of rows of workpieces transverse to the flow.

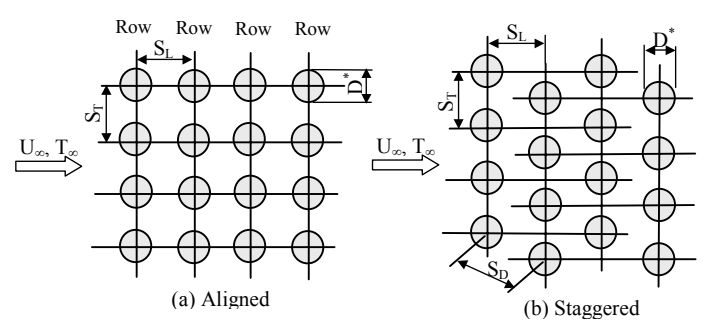

Figure 1.1-5. Configurations of arrays of workpieces

\subsubsection{Furnace model}

- Furnace temperature control model

The typical temperature control method in the furnace is PID controller (where PID stands for Proportional, Integral, and Derivative). When the measured temperature is different from the setting temperature, the heating or cooling input is controlled to minimize the error. It is a typical feedback control system as shown in Figure 1.1-6. 


\section{Section 1: Thermal Prediction}

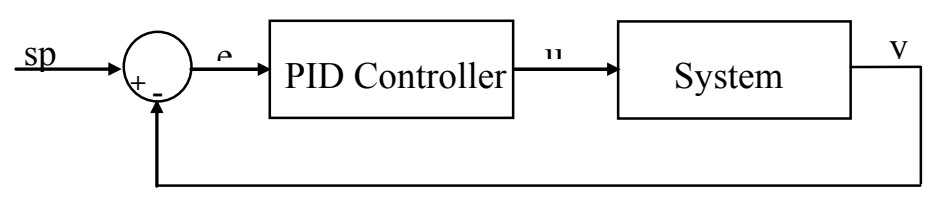

Figure 1.1-6. Control feedback loop of PID control method (where $\mathrm{sp} \rightarrow$ set point, $\mathrm{e} \rightarrow$ error, $\mathrm{u} \rightarrow$ output, $\mathrm{y} \rightarrow$ measured temperature

Generally PID method uses following equation to control the loop:

$$
u=P \cdot\left[\left(T_{s}-T_{o}\right)+D \cdot \frac{d}{d t}\left(T_{s}-T_{o}\right)+I \cdot \int\left(T_{s}-T_{o}\right) d t\right]
$$

where $T_{s}$ is the set point temperature, $T_{0}$ is the furnace temperature, the error value $e=\left(T_{s}-T_{0}\right) ; P$, $D$, and $I$ are known as proportional gain, damping, and integral gain, respectively.

In a $P I D$ control process, one of the most important things is to set the $P, D$, and $I$. Tuning these constants so that the weighted sum of the proportional, integral, and derivative terms produces a controller output that steadily drives the process variable in the direction required to eliminate the error. There are several methods that can be used to determine the $P, D$, and $I$ value. One of them is Ziegler and Nichols approach, which is a practical method of estimating the values of $K$, $T$, and $d$ experimentally. Here $K$ is the process gain used to represent the magnitude of the controller's effect on the process variable, $T$ is the process time constant used to represent the severity of the process lag, and $d$ is the dead-time used to represent another kind of delay present in many processes, where the "sensor" used to measure the process variable is located some distance from the "actuator" used to implement the controller's corrective efforts. With the controller in manual mode (no feedback), a step change is included in the controller output. Then the process reaction is analyzed graphically (Figure 1.1-7). 


\section{Section 1: Thermal Prediction}

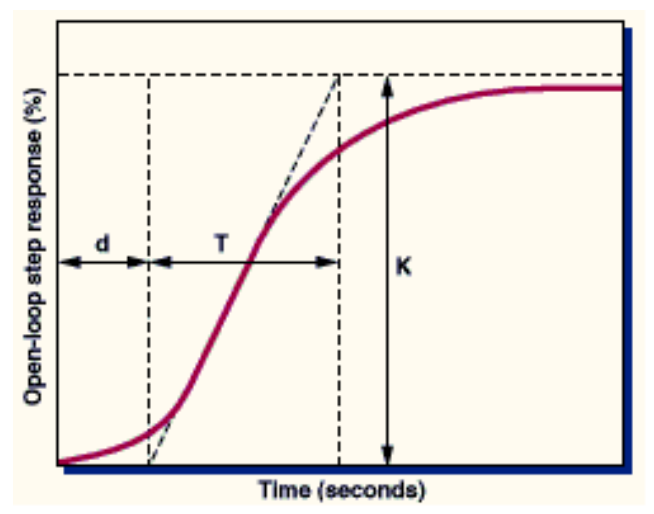

Figure 1.1-7. The Ziegler-Nichols Reaction Curve [2]

\subsubsection{System development}

The system consists of three main modules: pre-processing, heat transfer calculation and post-processing. The pre-processing is composed of sub-modules which include the 3-D solid geometrical module and finite difference enmeshment module. Furnace is of great importance in the simulation. However, it is dimensionally very large, of complicated structure and does not change with the load change. Therefore database for the 3-D furnace geometrical models have been established, including furnace walls, heating element and support. In the calculation the furnace CAD model is used directly so as to simplify the calculation and improve efficiency.

The conduction, radiation and furnace control mathematic models are integrated into the system. The system can cover eleven kinds of materials in total, such as parts, heater, furnace walls, muffle, baskets, supports, cooling air, cooling water and atmosphere. Some main interfaces of the software are shown in Figure 1.1-8 13. 


\section{Section 1: Thermal Prediction}

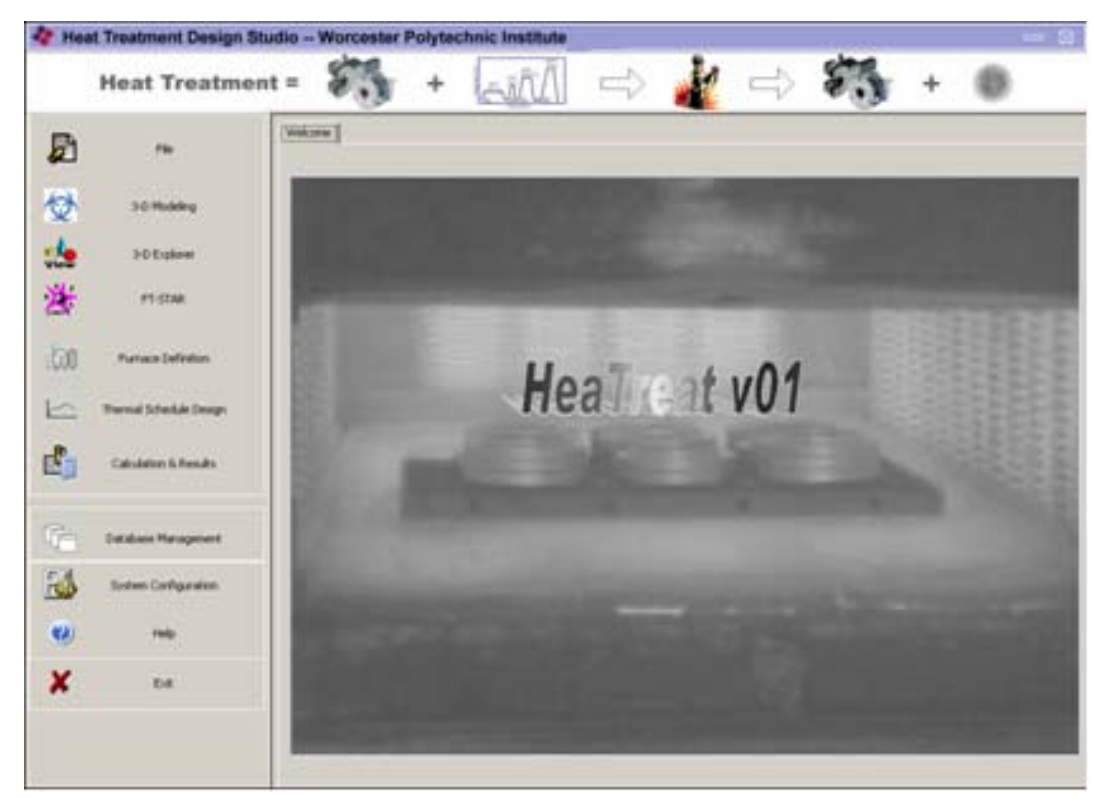

Figure 1.1-8. The system interface

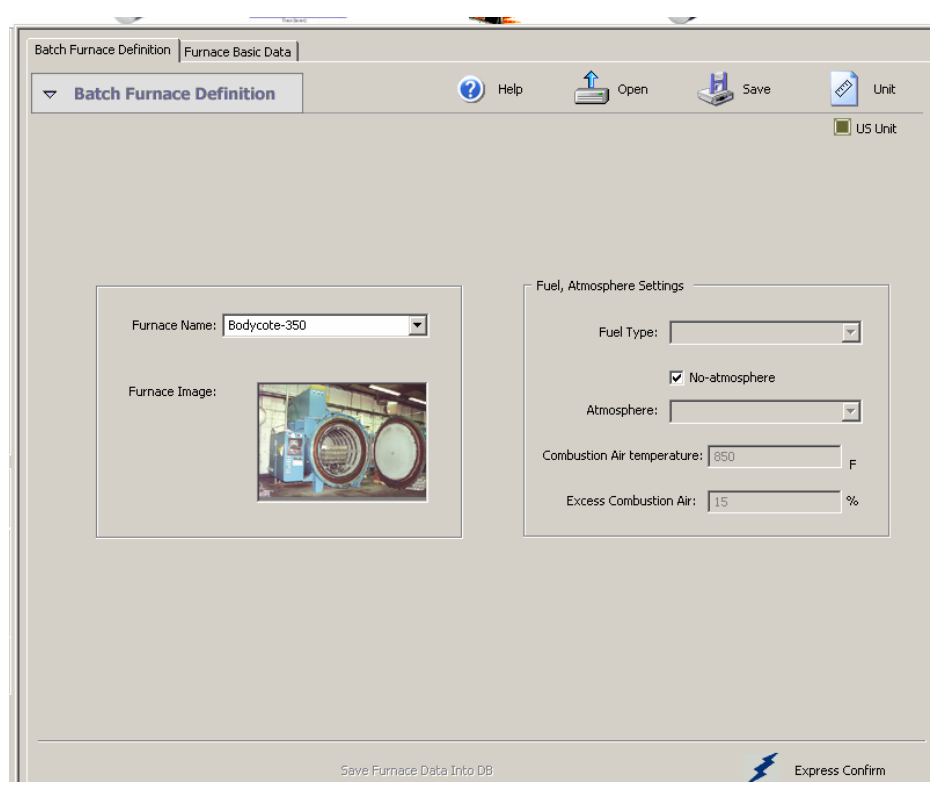

Figure 1.1-9. The furnace definition -page 1 


\section{Section 1: Thermal Prediction}

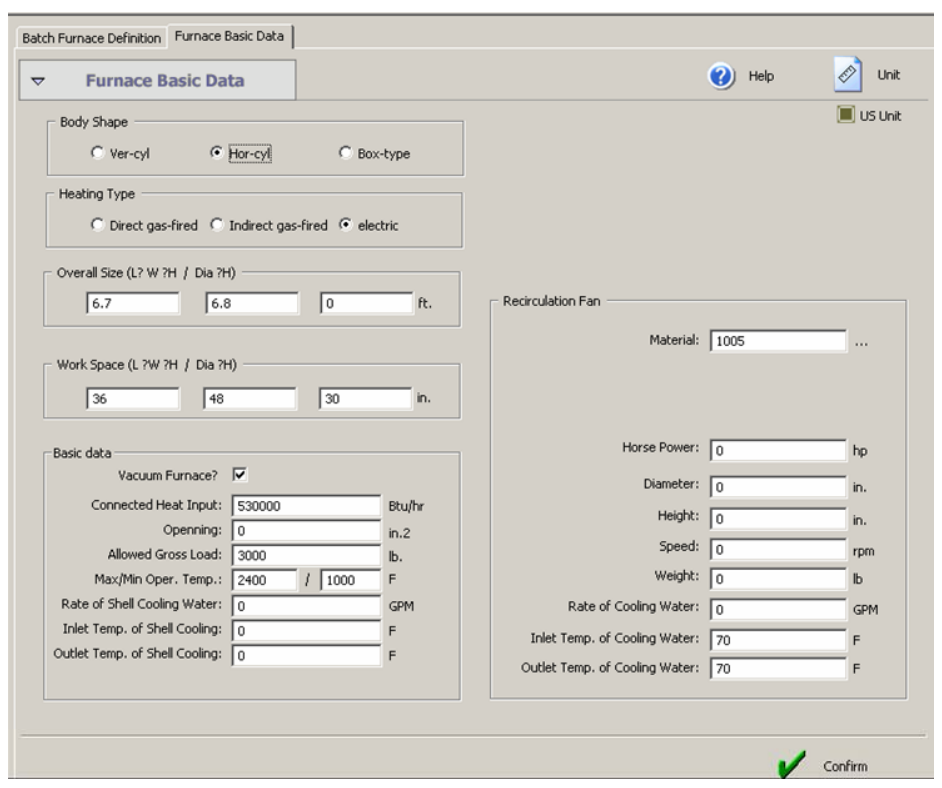

Figure 1.1-10. The furnace definition -page 2

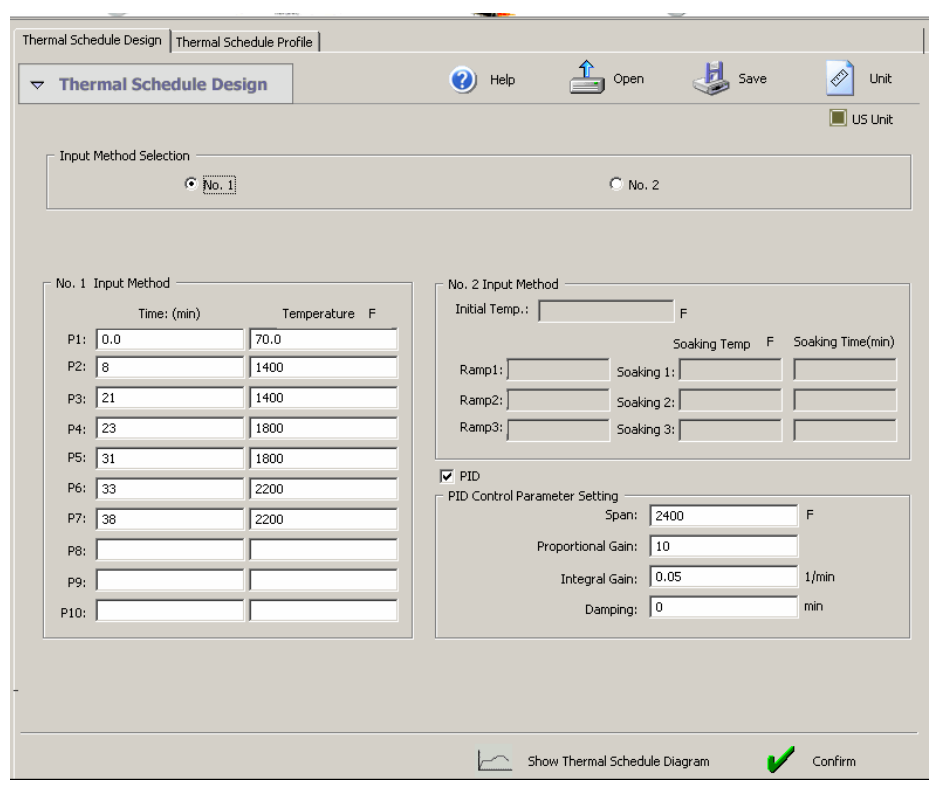

Figure 1.1-11. Thermal schedule dialog 


\section{Section 1: Thermal Prediction}

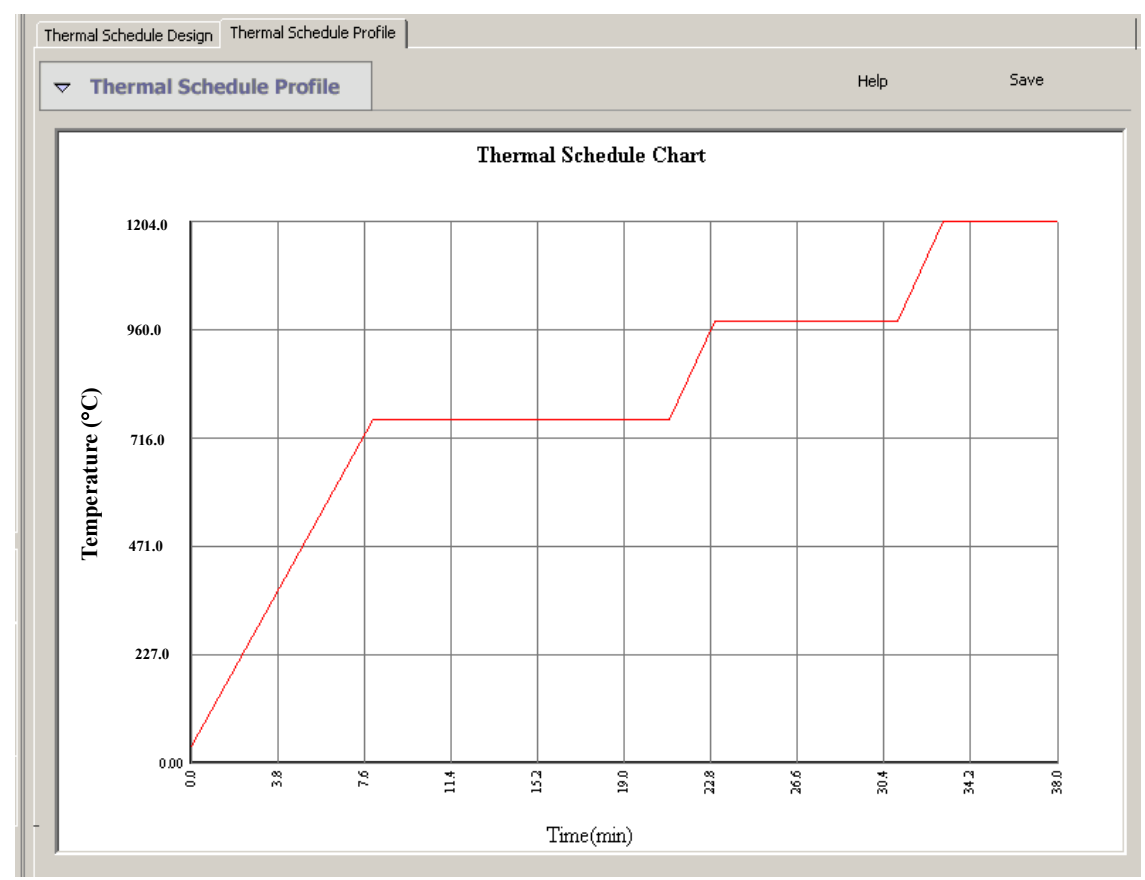

Figure 1.1-12. Thermal schedule chart

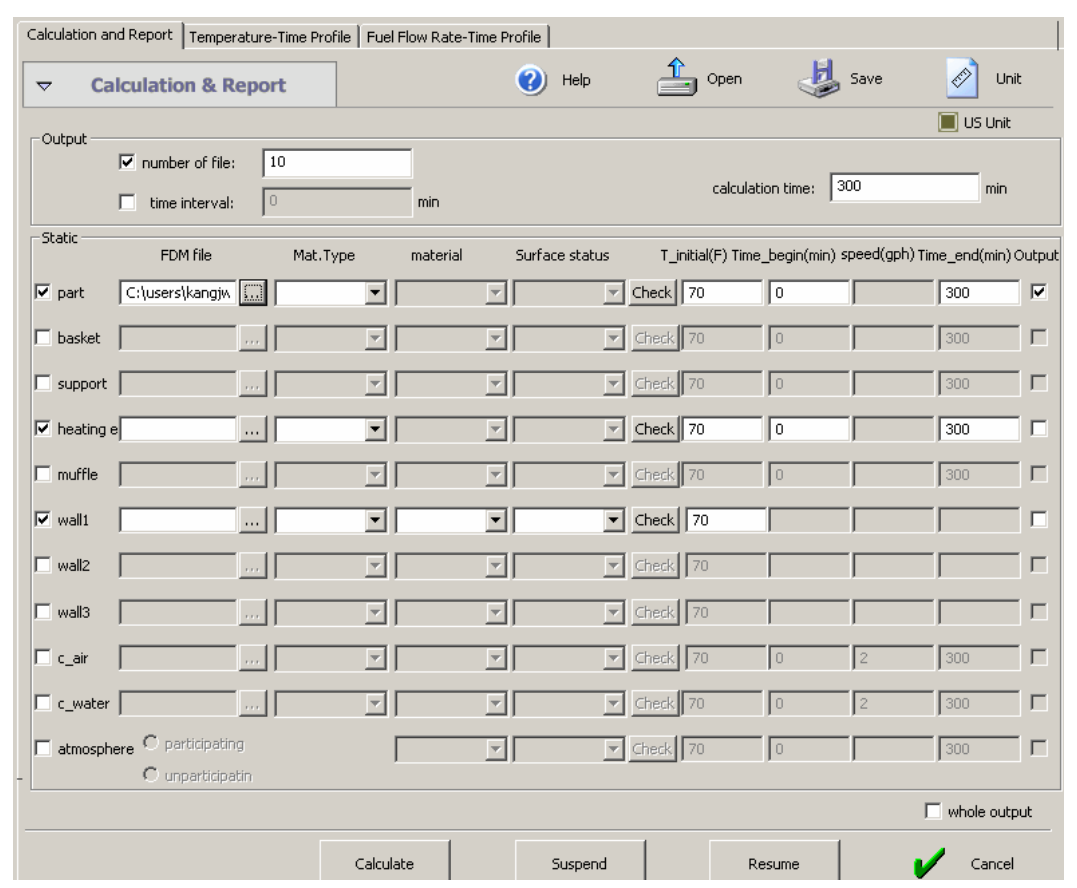

Figure 1.1-13. The calculation dialog 


\section{Section 1: Thermal Prediction}

\subsubsection{Validation of the system}

\section{- Case study 1}

Here a test example of steel material is presented because there is no difference in heat transfer model for the heating of steels and aluminum alloys. Eight blocks were located in a heat treatment furnace. Eight heating elements were presented in the furnace. The 3-D CAD model and finite difference model are shown in Figure 1.1-14 and Figure 1.1-15, respectively. In the two figures, three sides are cut off to see the inside special relationship. The calculated temperature distributions vs. time are shown in Figure 1.1-16 17. It can be seen form the results that the temperatures of the heating elements are far higher than those of workpieces and furnace walls. The system has been primarily validated by an example. Further validation can be conducted by real cases.

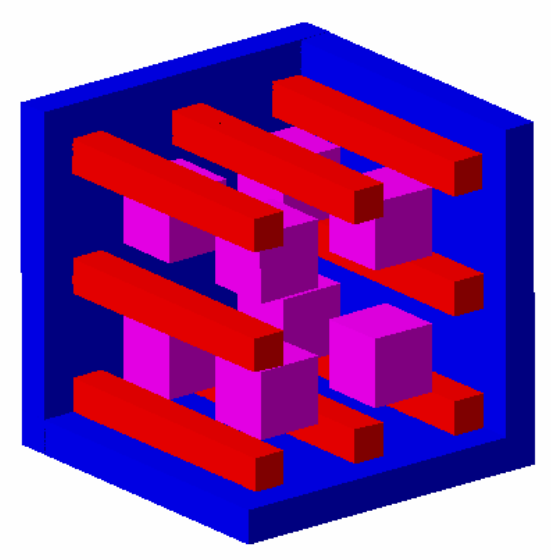

Figure 1.1-14. Geometrical model (partial; Figure Furnace wall, heating elements, parts)

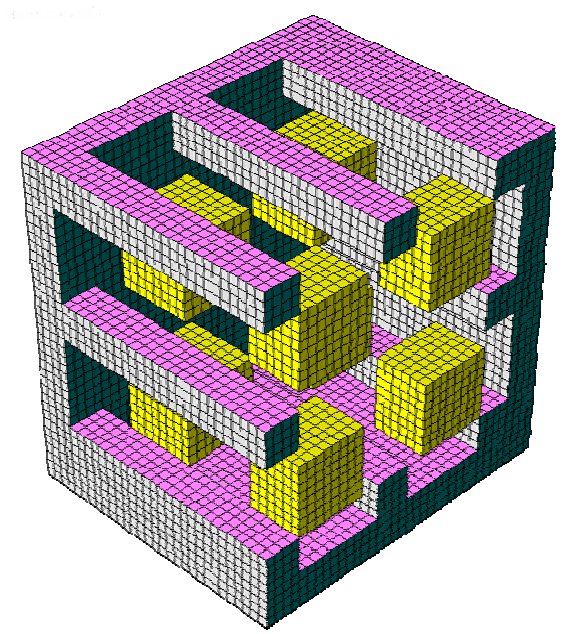
model(partial; Furnace wall, heating elements, parts) 


\section{Section 1: Thermal Prediction}

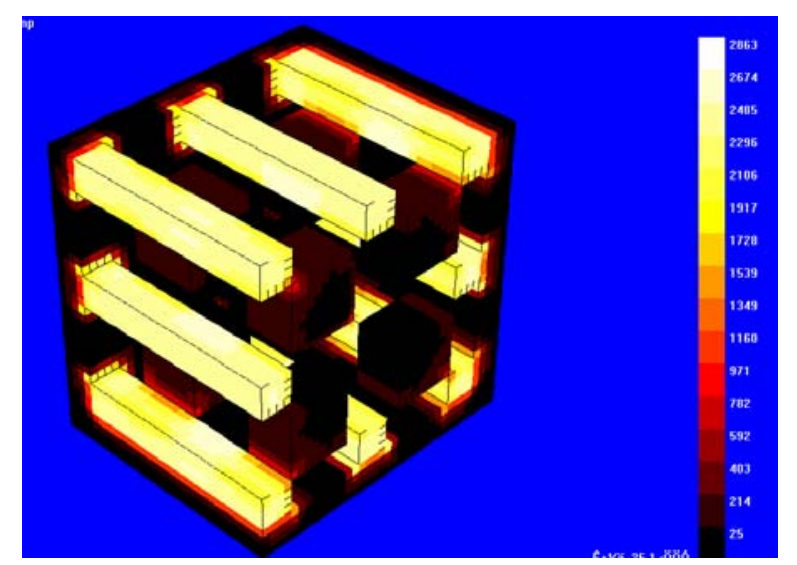

(a) 25 mins

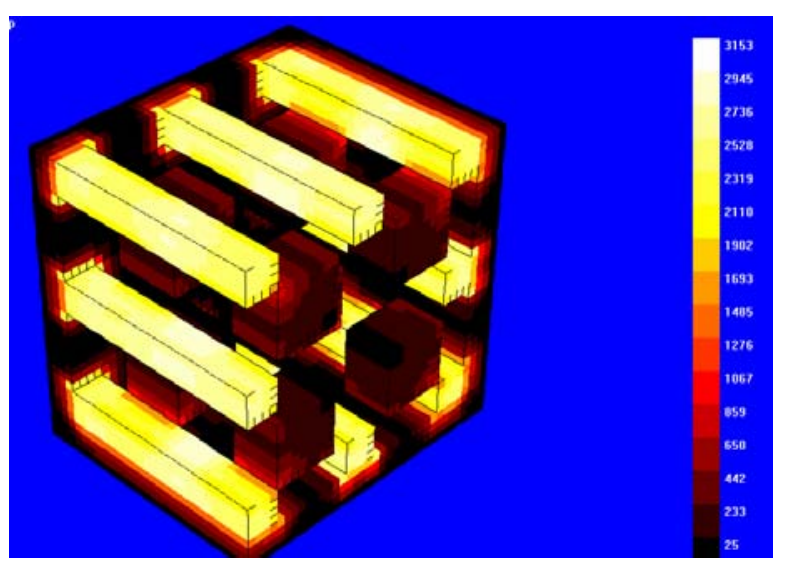

(b) $50 \mathrm{mins}$

Figure 1.1-16. Temperature distribution during heating

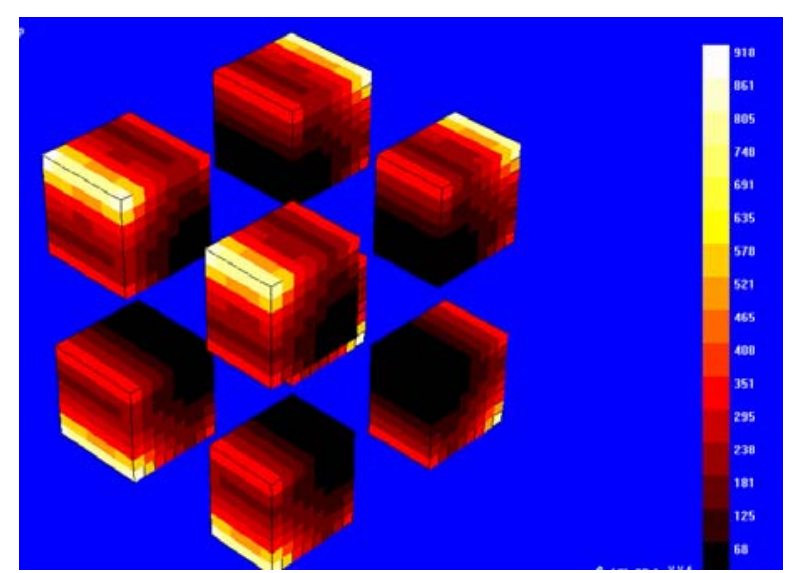

(a) 25 mins

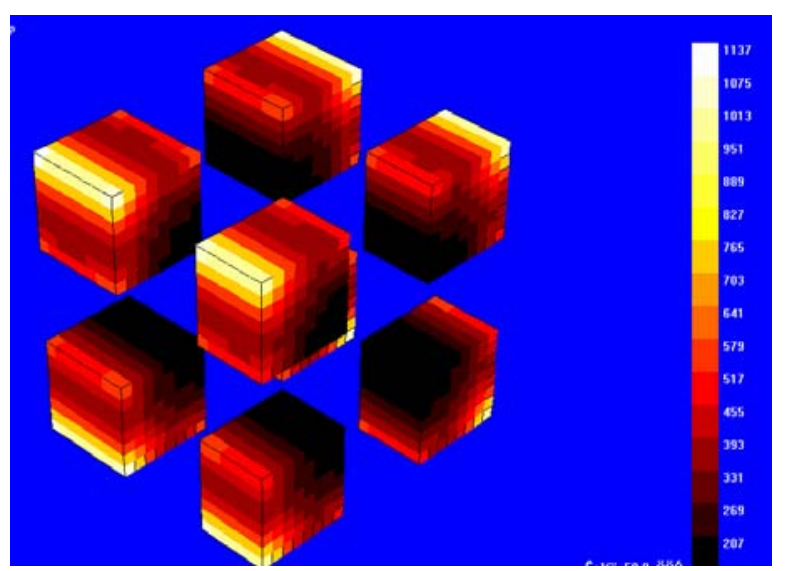

(b) $50 \mathrm{mins}$

Figure 1.1-17. Temperature distribution in parts during heating

\section{- Case study 2}

Another experiment has been done in the CHTE quenching laboratory to validate the system. The workpiece is of cylinder shape and with a blind hole was drilled at the center along the axis direction, as illustrated in Figure 1.1-18. The thermal couple was placed in the hole and touched 


\section{Section 1: Thermal Prediction}

the bottom. Graphite powder was used to stuff the gap in the hole to make sure good contact of the thermal couple and workpiece. This measurement method is similar to the probe used in the quenching tests. The furnace is FB1314M manufactured by Barnstead Thermolyne Coporation.
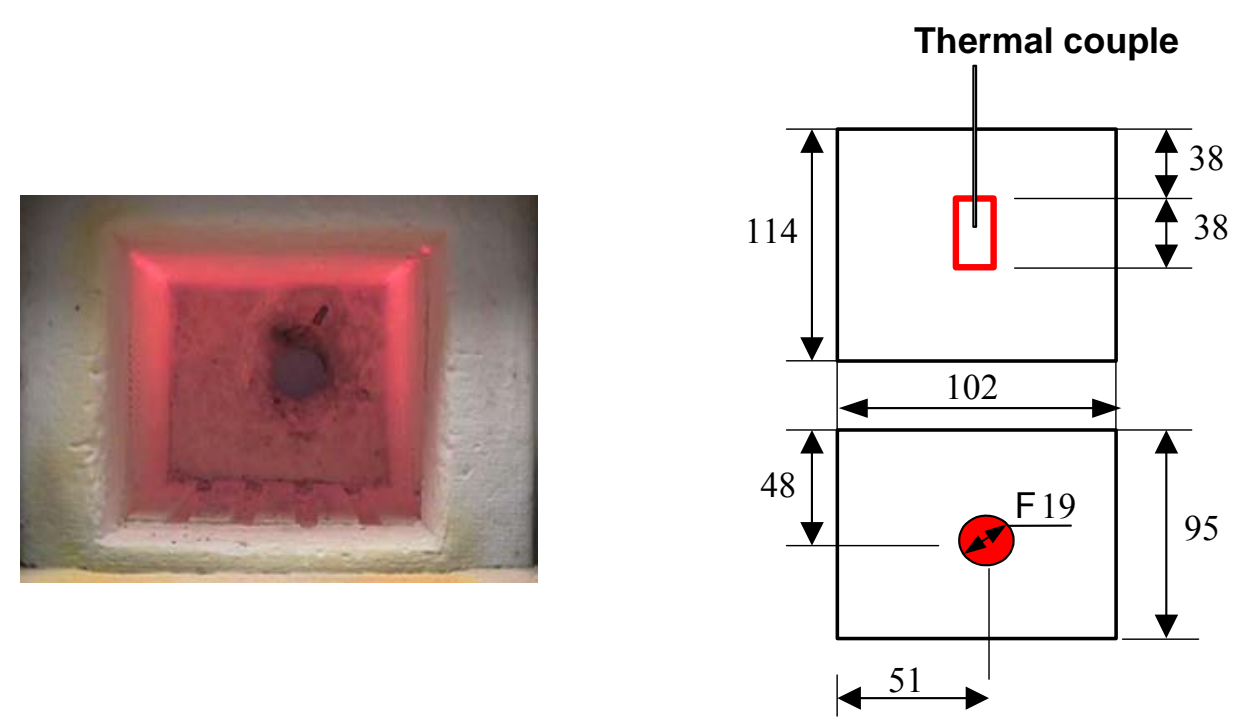

Figure 1.1-18. Workpiece and furnace

The view factor of an element $i$ of workpiece is given an example as shown in Figure 1.1-19. It can be seen that the view factor is distributed around the very opposite region and the elements with the same height, and the elements that its normal direction can touch. In the calculation, the view factor of each surface element to the other elements was calculated.

The temperature distribution of the whole furnace system at two different times are shown in Figure 1.1-20. It can be seen that the temperature of the workpiece, furnace wall and heaters are presented. Based on the thermal history of each item of the furnace, the whole process can be optimized.

In Figure 1.1-21 the calculated temperature and measured temperature are compared. They match very well except at the end period. The deviation at the end period is there because the furnace control coefficients are not available and no furnace temperature control is considered in the calculation. 


\section{Section 1: Thermal Prediction}

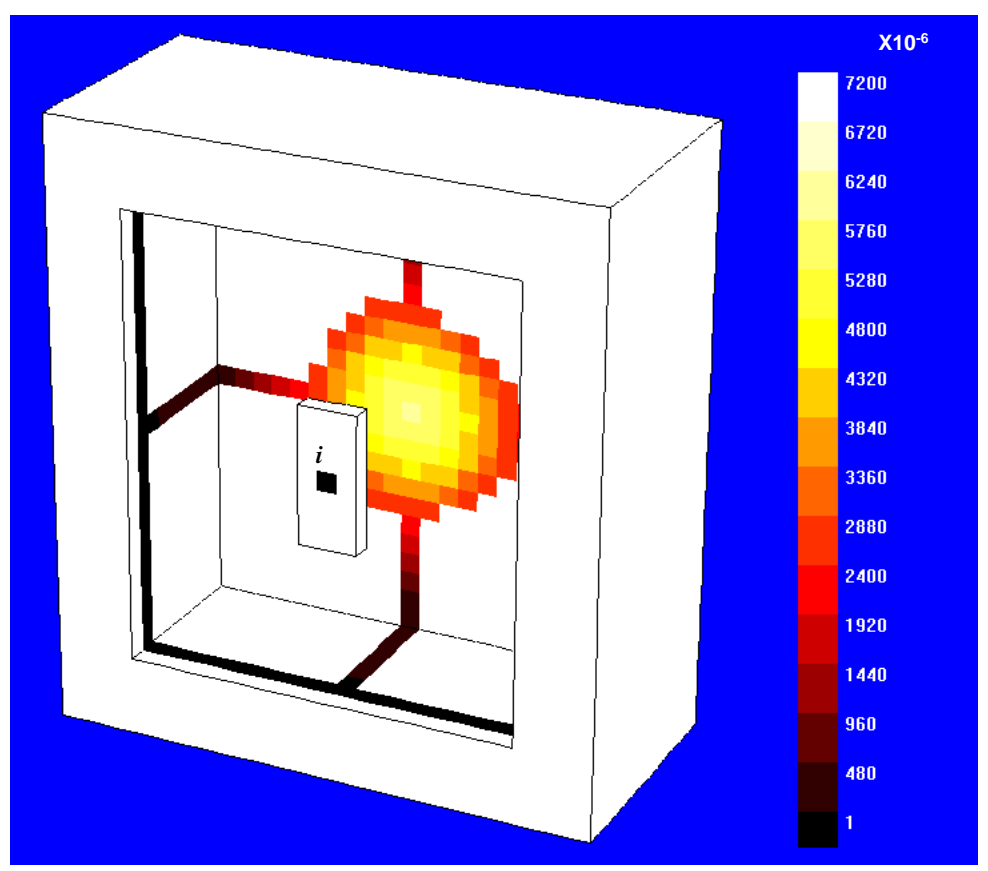

Figure 1.1-19. View factor distribution of surface element $I$

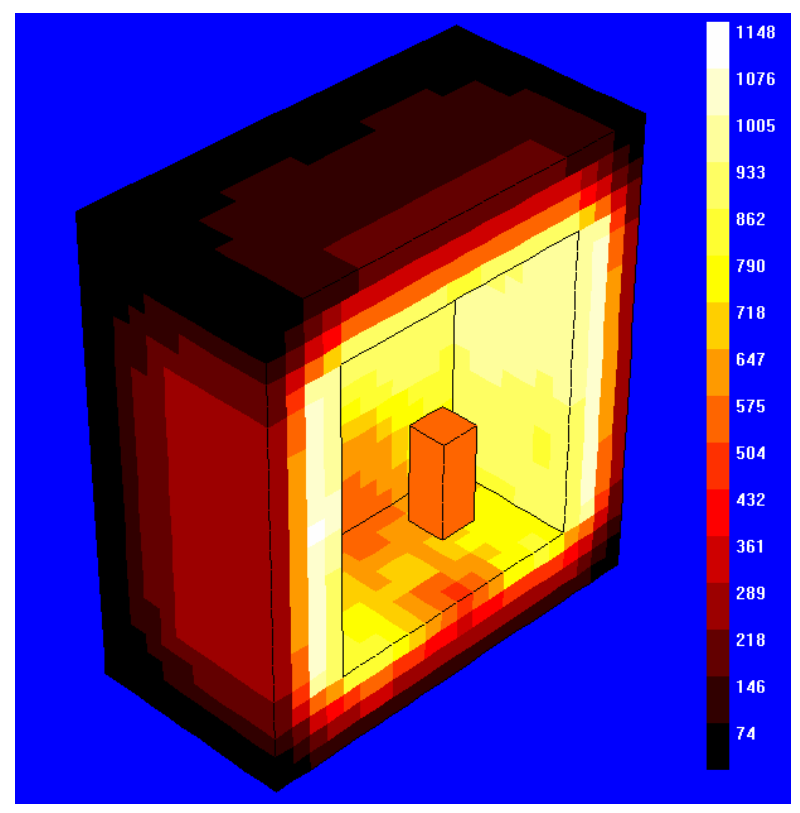

(a) $13 \mathrm{~min}$

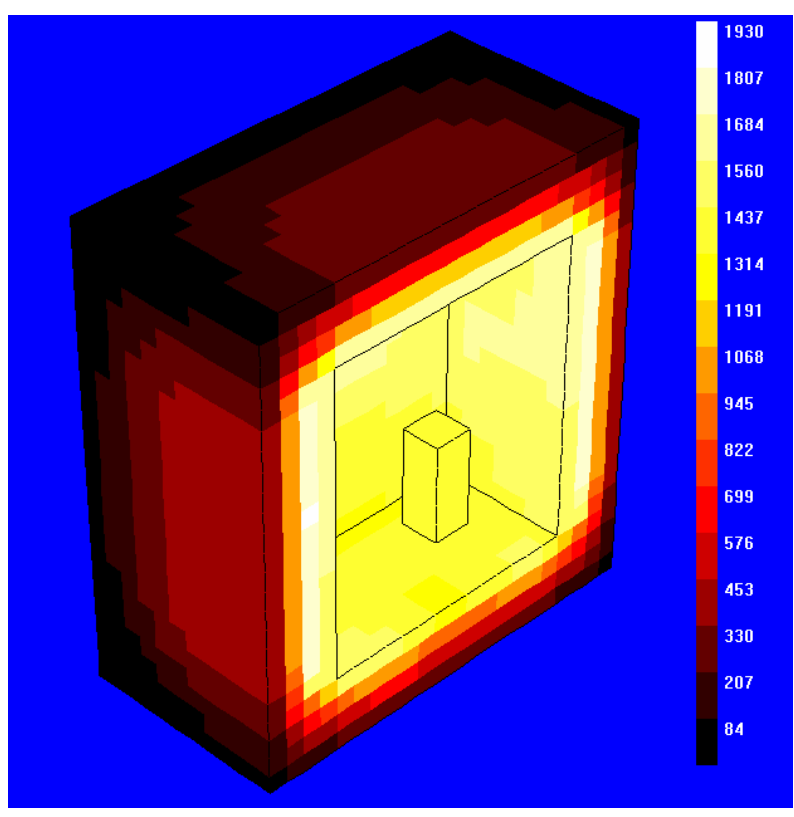

(b) $27 \mathrm{~min}$

Figure 1.1-20. Temperature distribution during heating process 


\section{Section 1: Thermal Prediction}

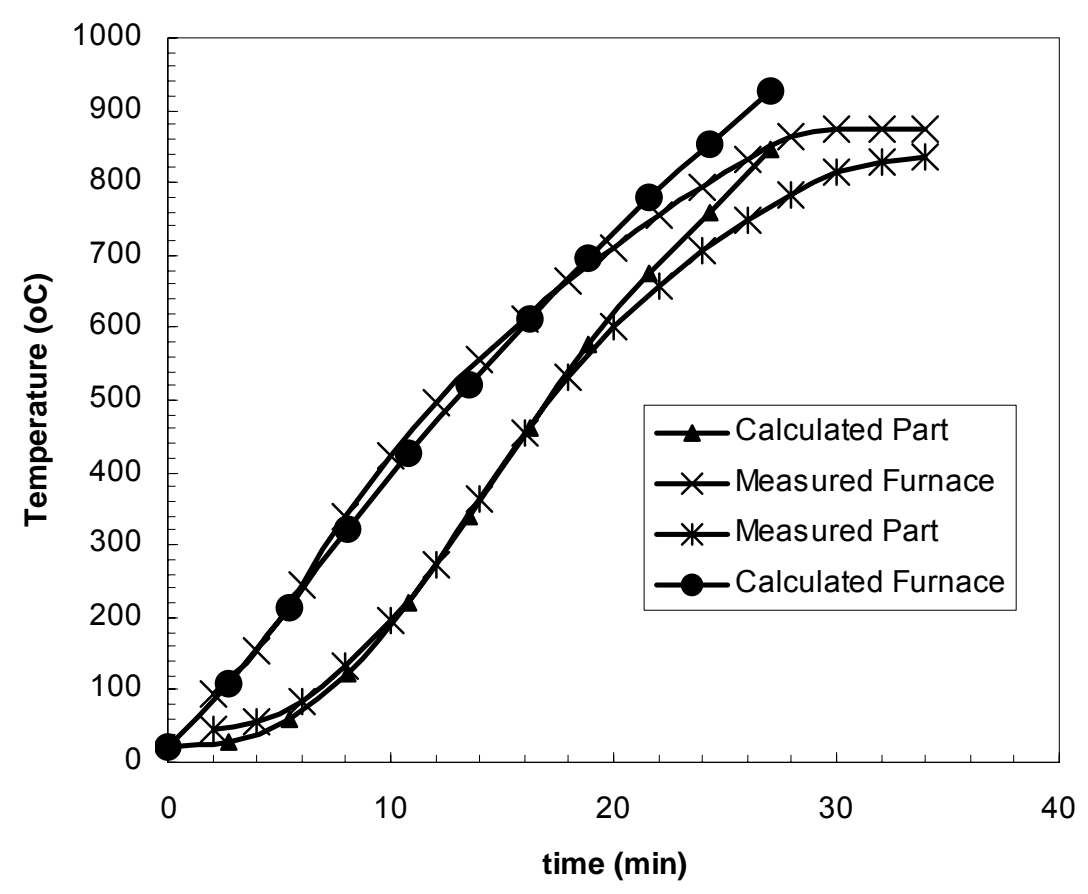

Figure 1.1-21. Comparison of calculated and measured temperature results of workpiece

\section{- Case study 3}

A five-cylinder cast aluminum A356 engine cylinder head (Figure 1.1-22) has been studied by the system. Thirty two parts were placed in the fixture with the arrangement of $2 \times 16 \times 1$, and 2 layers of fixture were loaded into the furnace. Then the engine heads were solutionized at $538^{\circ} \mathrm{C}$ $\left(1000^{\circ} \mathrm{F}\right)$ for 5 hours ( 7 hours including the ramp-up time), refer to Figure 1.1-23. The predicted results are plotted in Figure 1.1-24. 


\section{Section 1: Thermal Prediction}

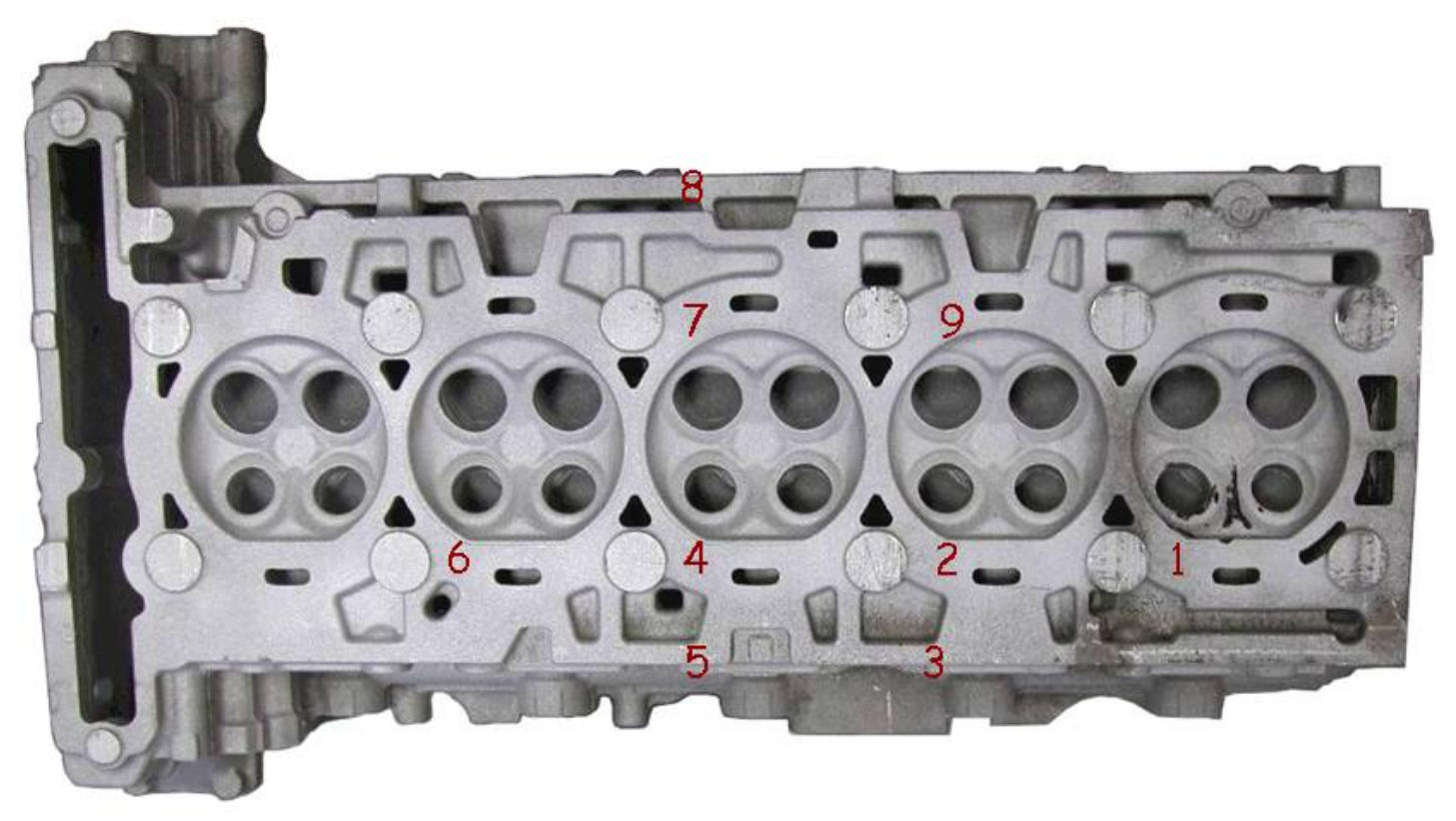

a) Photograph

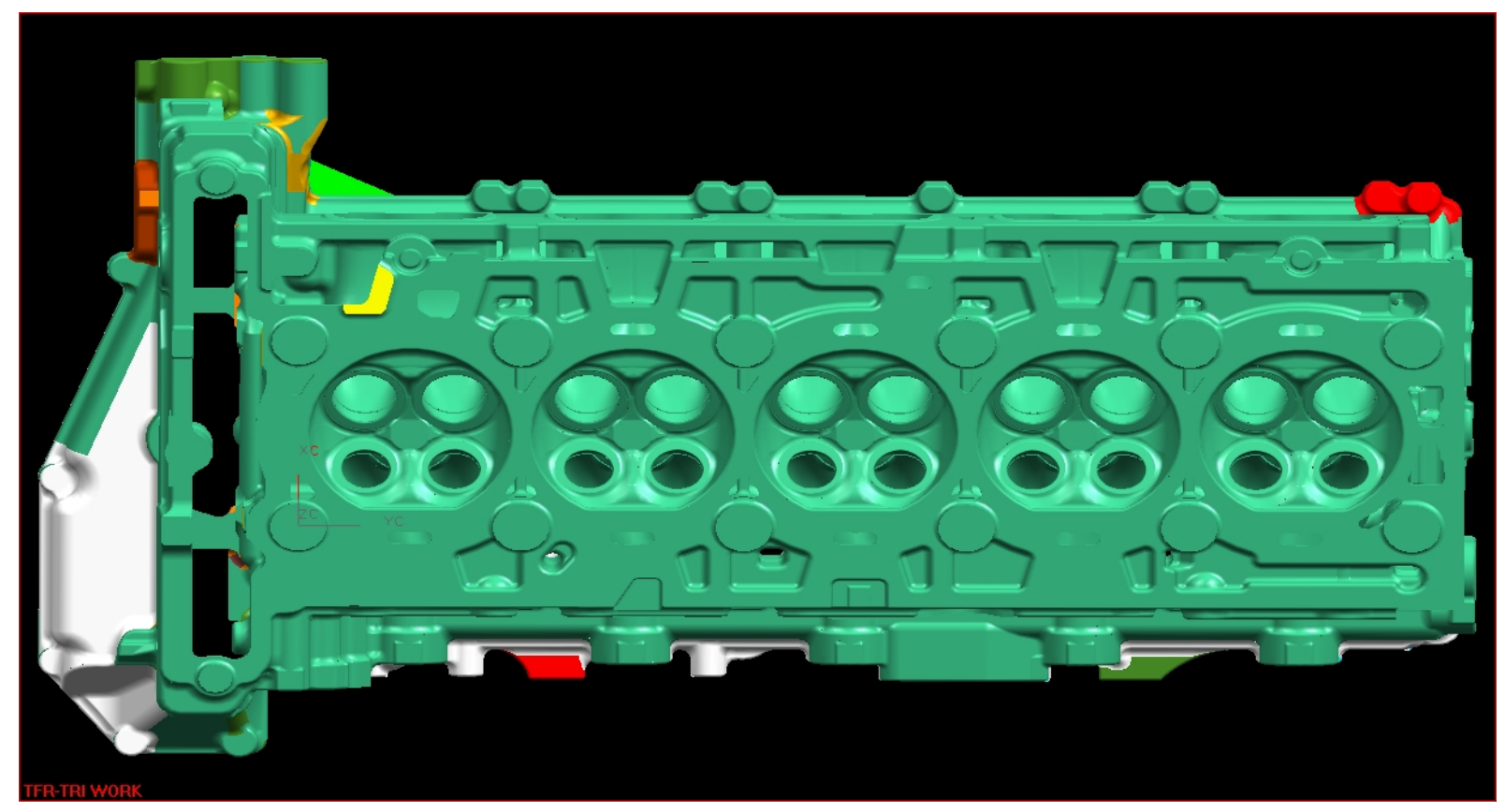

b) CAD model

Figure 1.1-22. Cast aluminum A356 engine head 


\section{Section 1: Thermal Prediction}

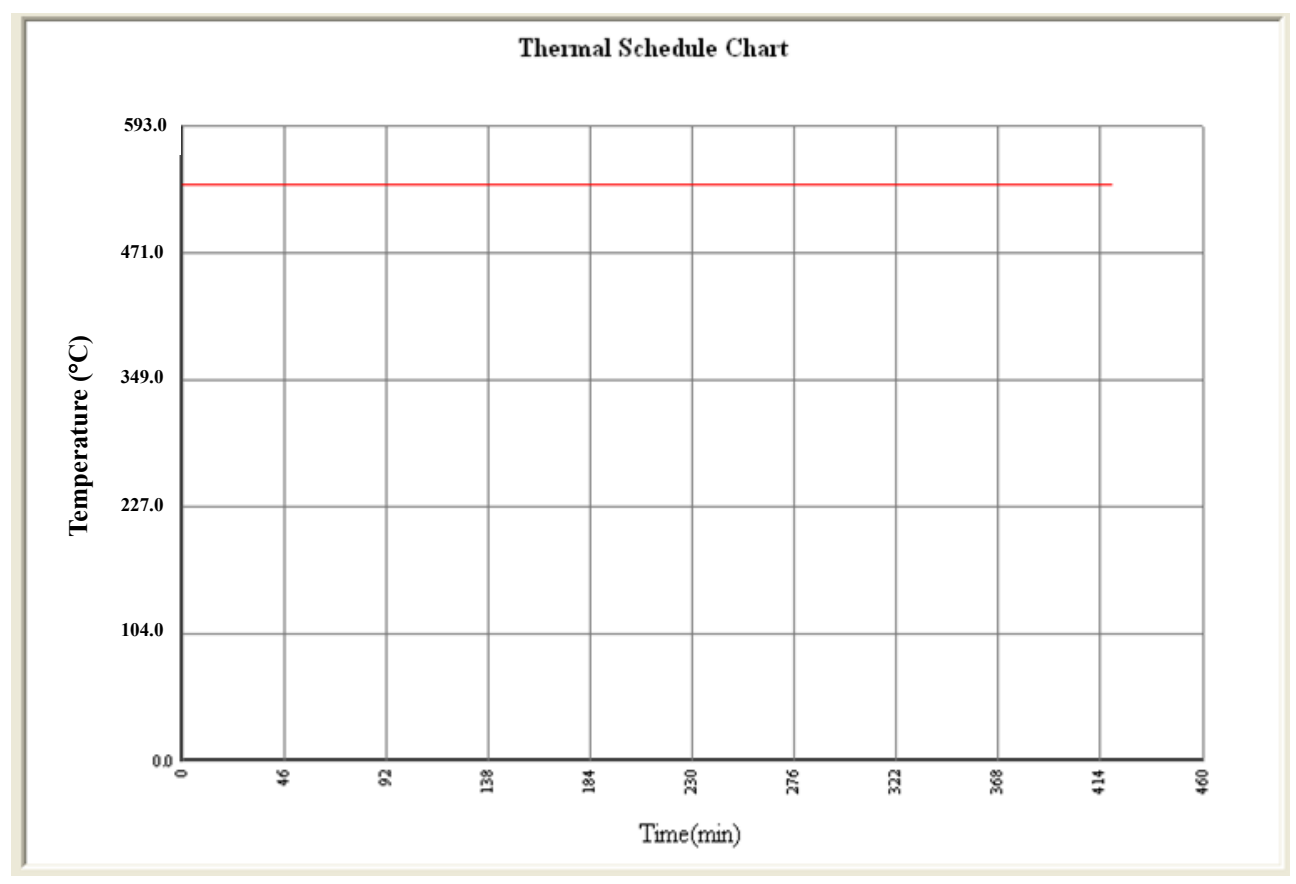

Figure 1.1-23. Thermal schedule

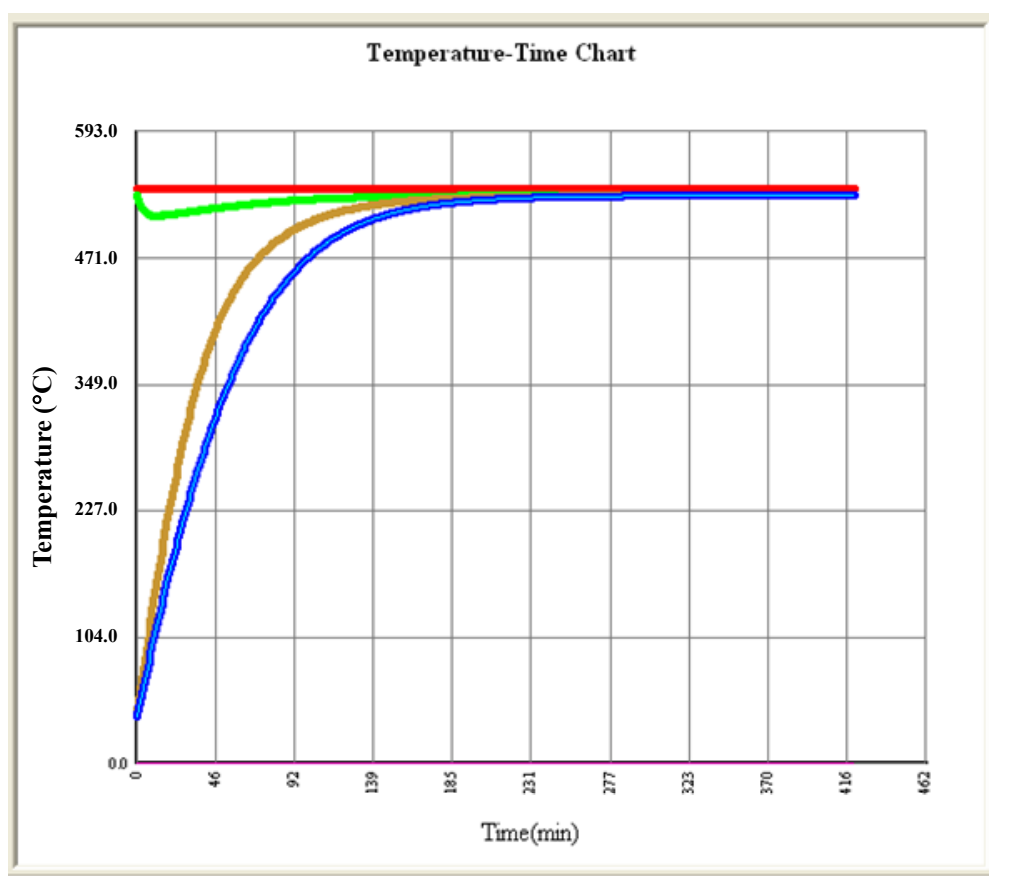

Figure 1.1-24. Predicted temperature profile of the load of engine heads (Unit: ${ }^{\circ} \mathrm{C}$ ) 


\section{Section 1: Thermal Prediction}

The predicted temperature profile of the load is displayed in Figure 1.1-24, while the red line presents set point, green line predicted furnace temperature, brown line heated fast part, and blue line heated slow part. It's shown that the whole load was uniformly heated up to the set point almost after 2 hours, and then solutionizing starts.

\subsection{OPTIMIZATION MODULES}

Based on HTFURNACE, two optimization modules are developed: PTLOAD and TPCONTROL. The objective of both modules is to reduce cycle time and energy consumption. PTLOAD explores the possibility of saving through load arrangement variation. For each load arrangement, PTLOAD extracts relevant process parameters based on the calculation in HTFURNACE to characterize the process performance in terms of cost. Heat treaters can then compare and determine between different load arrangements. TPCONTROL saves time and energy by adopting a new thermal cycle. The new thermal cycle is designed to heat the load faster. Algorithms are developed to determine the optimal thermal cycle and prevent overheating.

\subsubsection{PTLOAD}

Given several load arrangement designs, the thermal profile of each design is achieved through simulation. To quantitatively evaluate the process performance for each design, an objective function is defined, and additional process information related to the objective function is extracted from the simulation.

Since the goal for this approach is to reduce heat-up time and energy consumption without reducing the part quality of the parts after heat treatment, the objective function contains factors representing each of these criteria. The objective function is defined as in equation (1.2-1), which is a relative cost function on a per-unit-load-mass basis. One can then determine the load arrangement by choosing the one which minimizes relative cost function. 


\section{Section 1: Thermal Prediction}

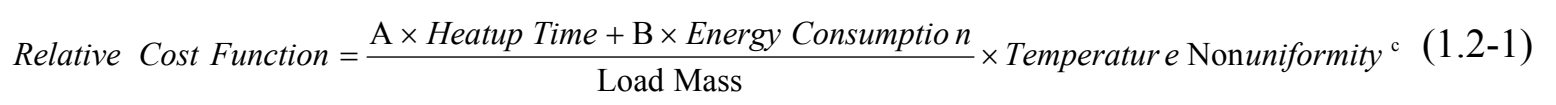

"Heat-up Time" is defined as the length of time during which the most slowly heated point of the load is heated from room temperature to the soaking temperature. "Energy Consumption" is defined as the total energy input to the furnace-load system from the start of heating to the time when the entire load reaches soaking temperature. "Temperature Nonuniformity" is defined as the maximum temperature difference within the load during heat-up period. "Temperature Nonuniformity" is a representative of the loss of overall quality or equivalent increase of cost. This is because the large temperature difference within the load will lead to higher risk of thermal stress or even crack in parts.

For different applications, these factors may be of different relative importance. The weight coefficients $\mathrm{A}, \mathrm{B}$ and $\mathrm{C}$ will allow varying allotment of importance among the factors. For the same application, different load arrangement should adopt the same value for the weight coefficients to achieve comparable results.

To examine the influence of load arrangement on process cycle time and energy consumption, two simulation cases have been set up for comparison. The two cases use same furnace, same thermal recipe, and same amount of same parts, but the parts were loaded differently in each case. The furnace model was created based on Lindberg Temperite $4.5 \mathrm{KW}$ box furnace. The part was a tensile bar made of Aluminum 319, and its shape and dimensions are shown in Figure 1.2-1. In both cases, 48 bars were loaded into 12 layers with 4 bars per layer. The difference was that the parts were aligned in one case and crisscross in the other case (Figures 1.2-2a and 1.2-2b). The thermal recipe is shown in Table 1.2-1.

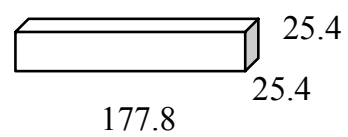

Figure 1.2-1. Shape and dimension of tensile bar (Unit: $\mathrm{mm}$ ) 


\section{Section 1: Thermal Prediction}

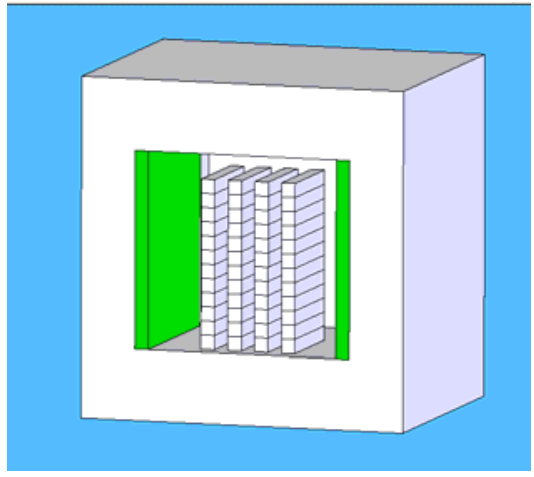

(a) Aligned load pattern

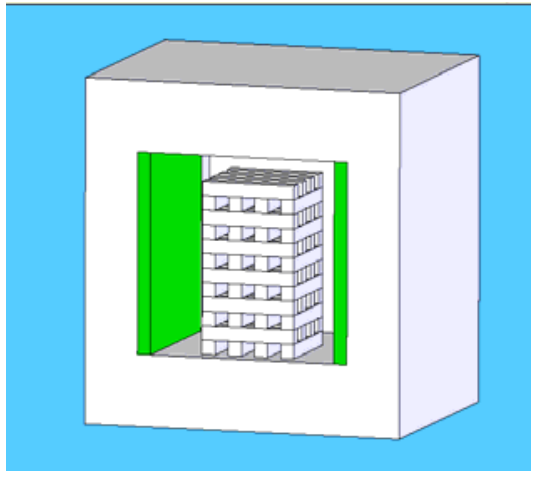

(b) Crisscross load pattern

Figure 1.2-2. Two different load patterns

Table 1.2-1. Thermal cycle variables and levels fore the simulation

\begin{tabular}{|l|l|l|l|}
\hline Time (min) & 0 & 40 & 520 \\
\hline Temperature $\left({ }^{\mathbf{0}} \mathbf{C}\right)$ & 158 & 1726 & 1726 \\
\hline
\end{tabular}

The temperature profile results of both cases are shown in Figures 1.2-3. It can be seen that the crisscross pattern had shorter heating period and less temperature difference between locations in comparison with the aligned pattern. Table 1.2-2 compares the process performance indicators of the two processes. As expected, the crisscross pattern has advantage over the aligned pattern for the given parts in terms of cycle time, energy consumption, temperature uniformity, and relative cost. The better performance in the crisscross pattern is attributed to the higher surface area which facilitates convective and radiative heat transfer. 


\section{Section 1: Thermal Prediction}

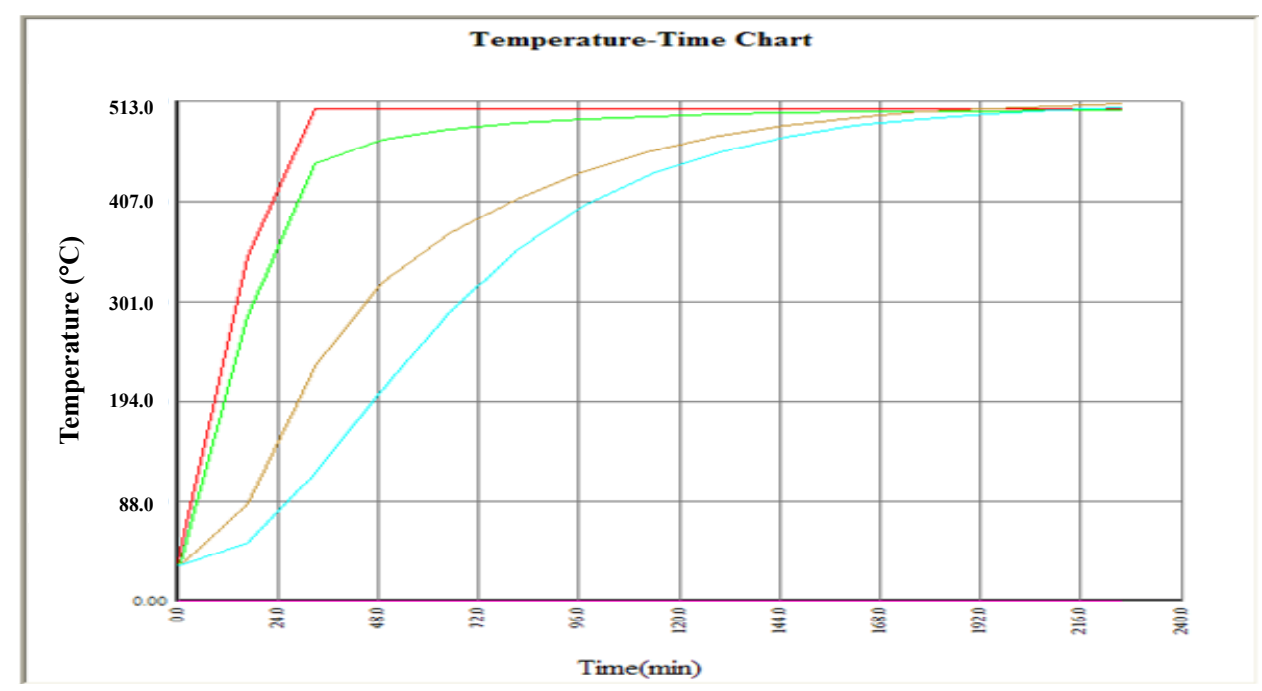

(a) Temperature profile of the aligned pattern

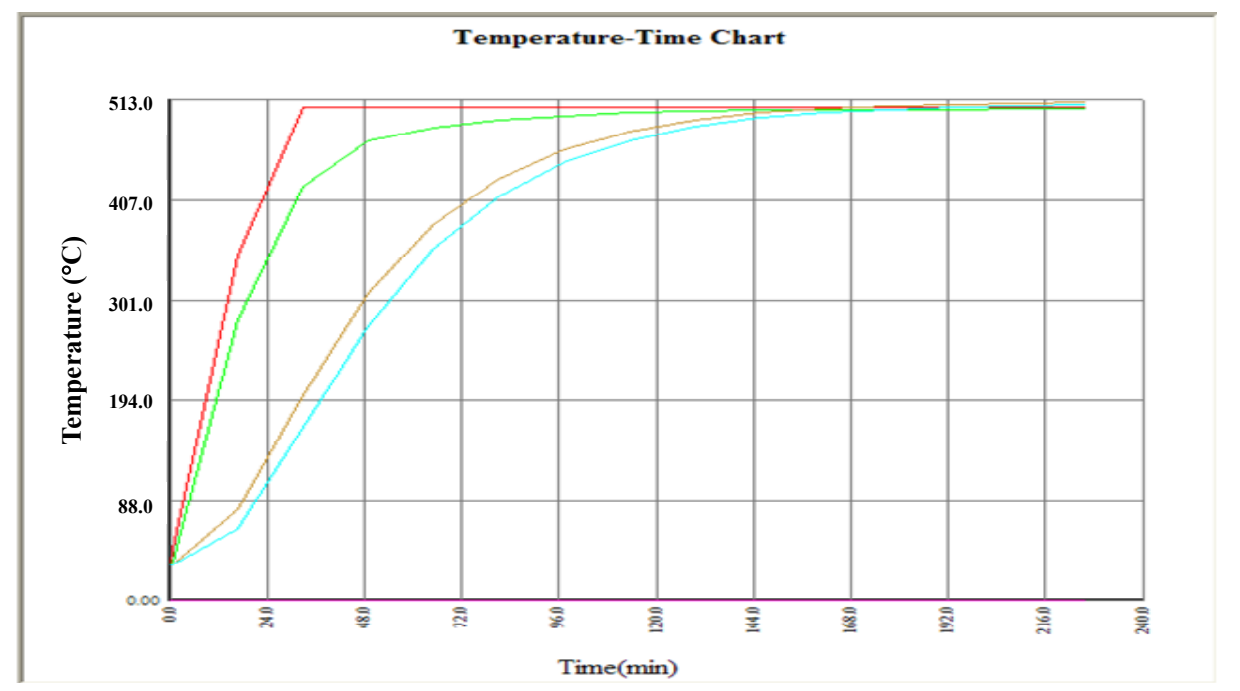

(b) Temperature profile of the crisscross pattern

Figure 1.2-3. Temperature profiles for two load arrangements. The four curves in each diagram from top to bottom represent thermal cycle, furnace temperature, temperature of the fastest heated point and the most slowly heated point of the load 


\section{Section 1: Thermal Prediction}

Table 1.2-2. Performance comparison between two load arrangements

\begin{tabular}{|l|l|l|l|l|l|}
\hline $\begin{array}{l}\text { Load Pattern } \\
\text { Design }\end{array}$ & $\begin{array}{l}\text { Load Mass } \\
(\mathbf{K g})\end{array}$ & $\begin{array}{l}\text { Heating up } \\
\text { Time } \\
(\mathbf{m i n})\end{array}$ & $\begin{array}{l}\text { Maximum } \\
\text { Temperature } \\
\text { Difference } \\
\left({ }^{\circ} \mathbf{C}\right)\end{array}$ & $\begin{array}{l}\text { Energy } \\
\text { Consumption } \\
(\text { KCal) }\end{array}$ & $\begin{array}{l}\text { Relative Cost } \\
\text { Function }\end{array}$ \\
\hline Aligned & 14.84 & 192.58 & 105 & 6551.5 & 23.78 \\
\hline Criss-cross & 14.84 & 162.79 & 19 & 6244.2 & 18.97 \\
\hline
\end{tabular}

Note: Coefficients $A=1, B=0.01, C=0.1$

\subsubsection{TPCONTROL}

In traditional heating process, furnace is heated from room temperature directly to soaking temperature (Figure 1.2-4). To heat the load faster, however, thermal cycle can be such designed that the furnace is first heated to and kept at an elevated temperature above soaking temperature (Figure 1.2-5). After a "proper" length of time, the furnace is shut off, and it releases heat until the furnace temperature decreases to the soaking temperature. At the same time, the load absorbs heat and the load temperature will keep increasing.

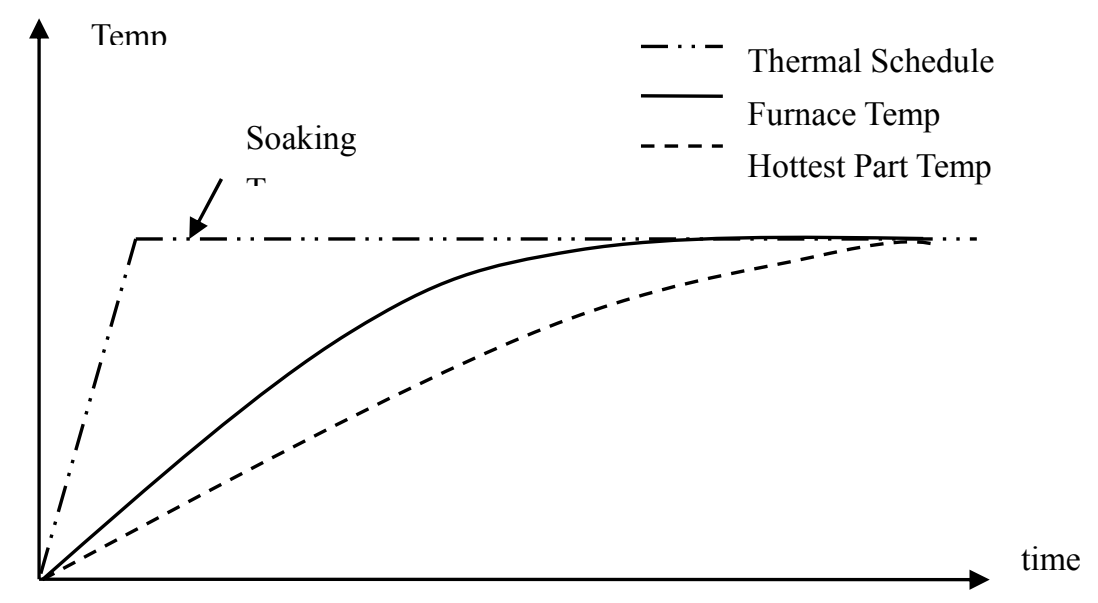

Figure 1.2-4. Traditional thermal cycle 


\section{Section 1: Thermal Prediction}

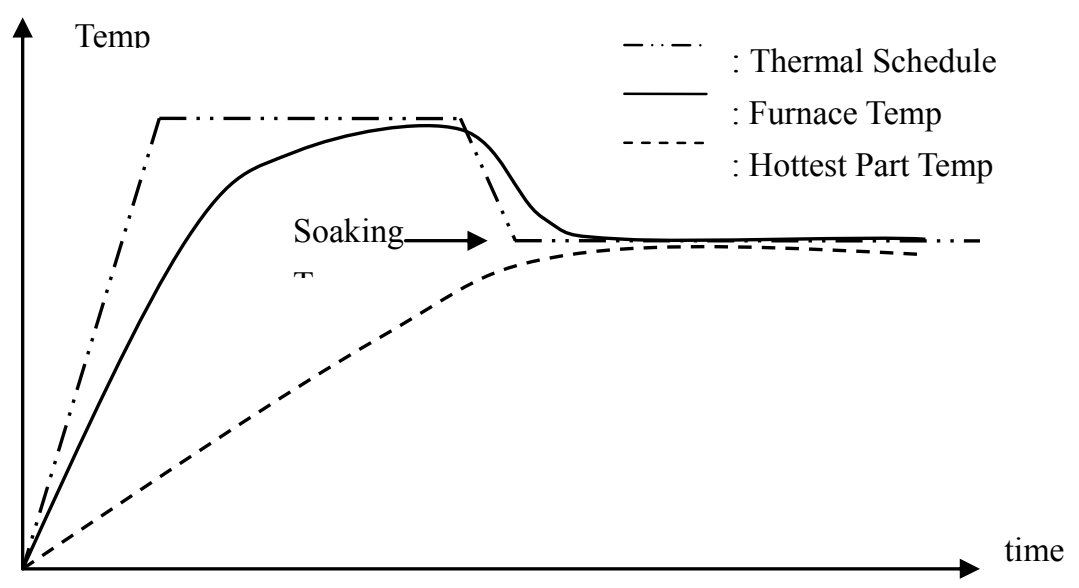

Figure 1.2-5. New thermal cycle design

The longer the furnace is kept at the elevated temperature before shut-off, the faster the load temperature rises. However, if the furnace is shut off too late, the temperature of part of the load will increase to the level of overheating, which is strictly prohibited. Thus it is critical to determine the proper length of time during which the furnace is kept at the elevated temperature.

Given room temperature, heat-up rate, the elevated temperature, and the soaking temperature, the problem becomes to determine the furnace shut-off time. With both the considerations of fast heating and prevention of overheating, the most desirable furnace shut-off time is such that after the furnace is shut off, the heat absorbed by load happens to suffice the fastest heated part of the load to just reach but not exceed the soaking temperature.

A numerical based iterative algorithm is designed to determine the "proper" furnace shut-off time. The algorithm takes room temperature, heat-up rate, the elevated temperature, and the soaking temperature as inputs. In the initial iteration, the furnace is set to be shut off when the fastest heated part of the load reaches soaking temperature. This furnace shut-off time is obviously too late because the temperature of the entire load will still increase and overheating of load will occur. However, through simulation we can get the maximum load temperature in the heating history, and hence know how much the load exceeds the soaking temperature. Through 


\section{Section 1: Thermal Prediction}

equation (1.2-2), this excessive temperature is translated into an excessive furnace heating time. Subtracting this excessive furnace heating time from the old furnace shut-off time generates a new furnace shut-off time, which is earlier. In the next iteration, simulation starts from the new furnace shut-off time, while using the same thermal profile before this time point. In the new iteration, the maximum load temperature is lowered as the furnace is shut off earlier. Then, furnace shut-off time is correspondingly updated again using the same method (Figure 1.2-6). The iteration continues until the maximum load temperature no longer exceeds the soaking temperature. After the iteration terminates, the furnace shut-off time achieved from the final simulation is the desired shut-off time. The algorithm is shown in flowchart (Figure 1.2-7).

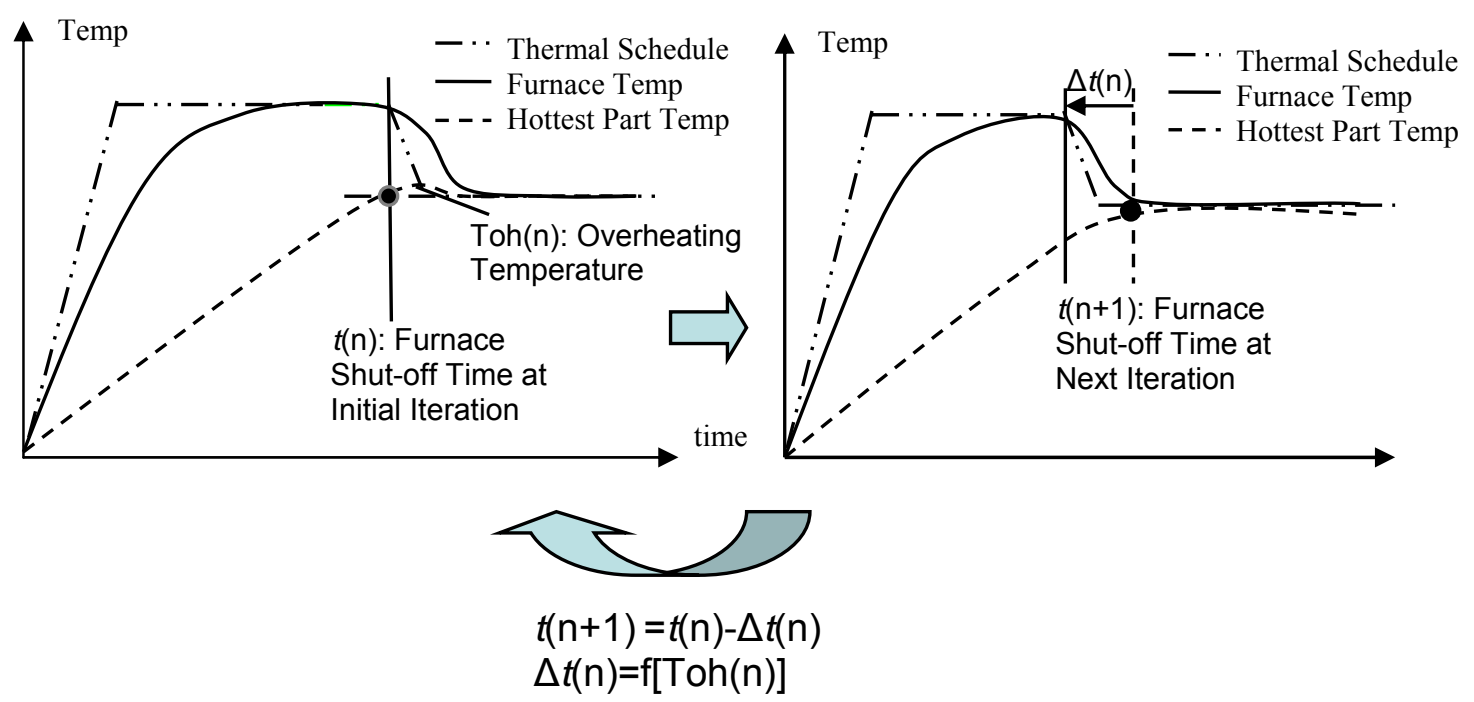

Figure 1.2-6. Calculation of furnace shut-off time

$$
T_{l}\left[t_{f}^{s}(n+1)\right]=T_{l}\left[t_{f}^{s}(n)\right]-\left(T_{l}^{\max }-T_{s}\right) \times \frac{T_{f}^{h}-T_{s}}{T_{f}^{h}-T_{l}^{\max }}
$$

$T_{1}$------ Load temperature

$t_{f}^{\mathrm{s}}(\mathrm{n})$--- Furnace shut-off time in $\mathrm{n}$-th iteration

$\mathrm{T}_{1}^{\max }$--- Maximum load temperature

$\mathrm{T}_{\mathrm{s}}$------ Soaking temperature

$\mathrm{T}_{\mathrm{f}}^{\mathrm{h}}$----- Elevated temperature the furnace is first kept at 


\section{Section 1: Thermal Prediction}

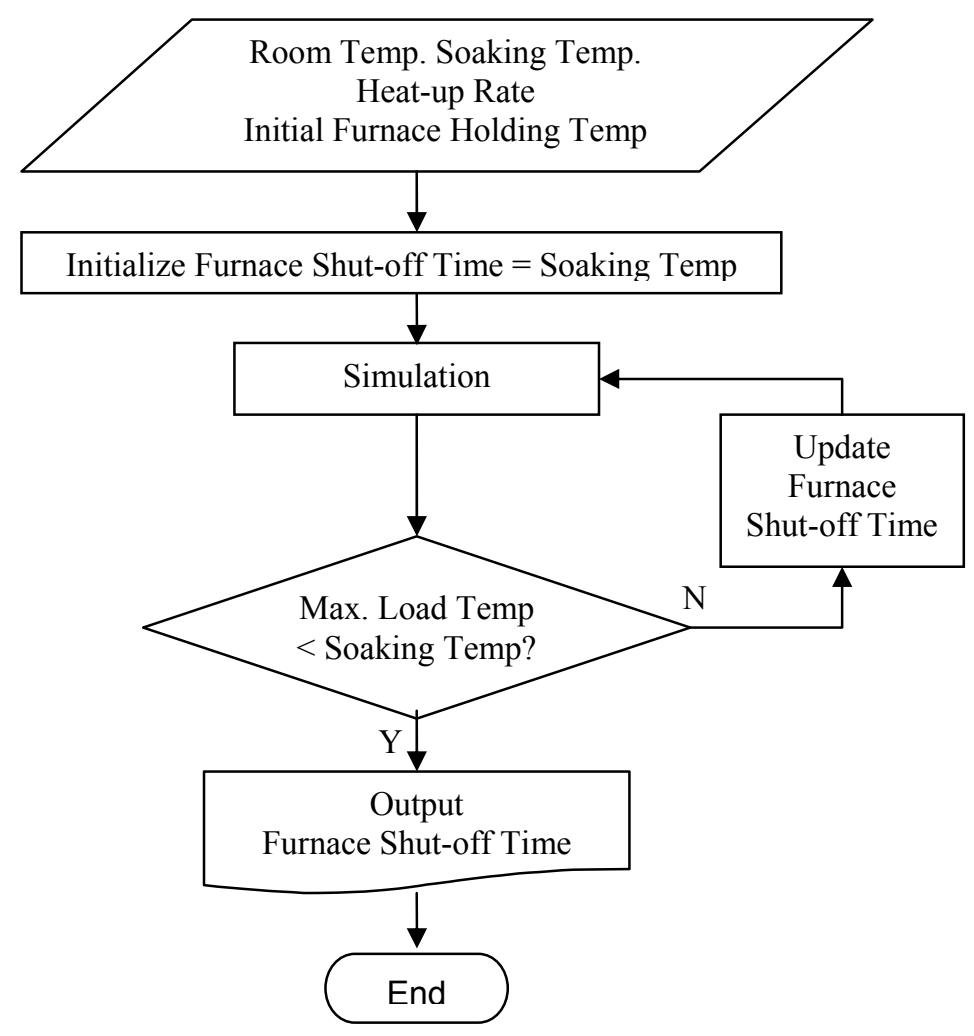

Figure 1.2-7. Flowchart of the iterative algorithm

A simulation case has been carried out to show the effectiveness of the proposed new thermal cycle. In this simulation, the part is the same as the one used in PTLOAD simulation cases, and the load is set up in the crisscross pattern. Table 1.2-3 shows the input data for the thermal cycle simulation including the specified room temperature, soaking temperature, elevated furnace temperature, and heating rate.

Table 1.2-3 Input data for thermal recipe optimization

\begin{tabular}{|c|c|c|c|}
\hline $\begin{array}{c}\text { Room Temperature } \\
\left({ }^{\circ} \mathbf{C}\right)\end{array}$ & $\begin{array}{c}\text { Soaking Temperature } \\
\left({ }^{\circ} \mathbf{C}\right)\end{array}$ & $\begin{array}{c}\text { Elevated Furnace } \\
\text { Temperature }\left({ }^{\circ} \mathbf{C}\right)\end{array}$ & $\begin{array}{c}\text { Heating Rate } \\
\left({ }^{\circ} \mathbf{C} / \mathbf{m i n}\right)\end{array}$ \\
\hline 21 & 505 & 593 & 2 \\
\hline
\end{tabular}




\section{Section 1: Thermal Prediction}

After simulation, the temperature profile of the process is achieved, as shown in Figure 1.2-8. The calculated desirable furnace shut-off time is $83.09 \mathrm{~min}$. The simulation results also indicate that if furnace is shut off at this time the maximum load temperature during the whole heat-up process will not exceed $505{ }^{\circ} \mathrm{C}$, which complied with the quality requirement.

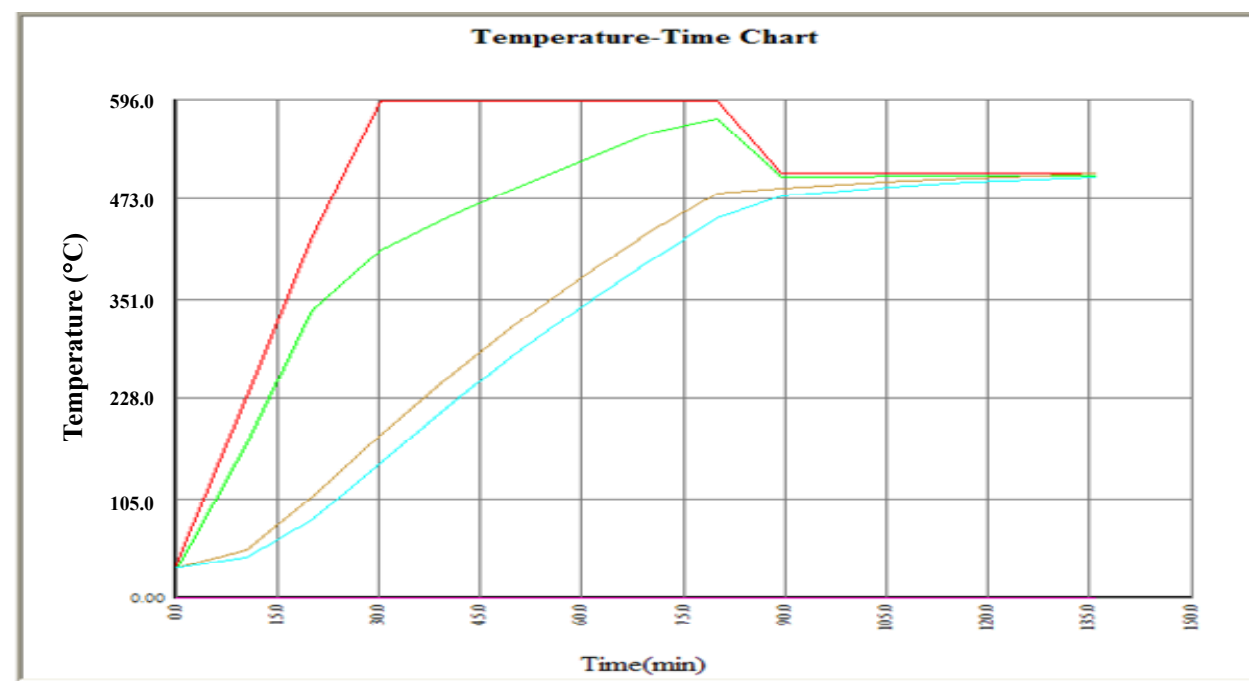

Figure 1.2-8. Temperature profile using the new thermal recipe. The four curves in each diagram from top to bottom represent thermal cycle, furnace temperature, temperature of the fastest heated point and the most slowly heated point of the load

To compare the new thermal cycle with the traditional one in which the furnace is heated up directly to the soaking temperature, the simulation has been conducted using the traditional thermal cycle. The temperature profile for the traditional thermal cycle is shown in Figure 1.2-9. The cycle time and energy consumption in both processes are listed in Table 1.2-4. As a result of adopting the new thermal cycle, the cycle time is reduced by $16 \%$ and energy consumption was reduced by $12 \%$. 


\section{Section 1: Thermal Prediction}

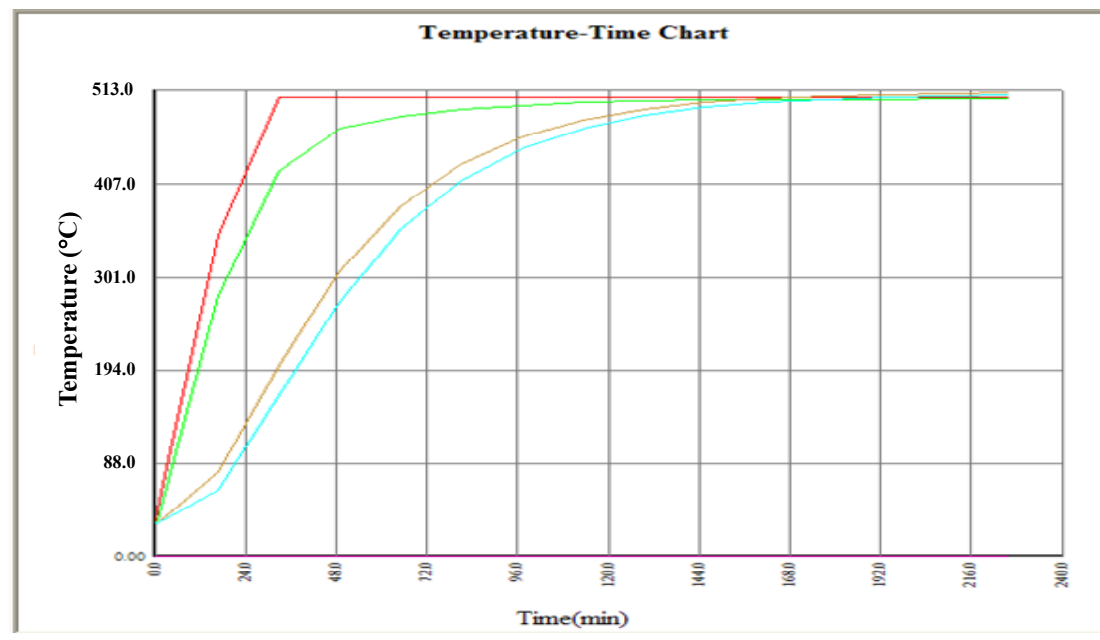

Figure 1.2-9 Temperature profile using traditional thermal cycle. The four curves in each diagram from top to bottom represent thermal cycle, furnace temperature, temperature of the fastest heated point and the most slowly heated point of the load

Table 1.2-4. Comparison between the traditional and new thermal cycles

\begin{tabular}{|c|c|c|c|}
\hline & $\begin{array}{c}\text { Heating Time } \\
(\mathbf{m i n})\end{array}$ & $\begin{array}{c}\text { Energy Consumption } \\
(\mathbf{K C a l})\end{array}$ & $\begin{array}{c}\text { Maximum Temp Diff } \\
\left({ }^{\circ} \mathbf{C}\right)\end{array}$ \\
\hline $\begin{array}{c}\text { Traditional thermal } \\
\text { recipe }\end{array}$ & 162.79 & 6224.2 & 18.61 \\
\hline New thermal recipe & 136.7 & 5470.6 & 18.70 \\
\hline
\end{tabular}

Two optimization modules PTLOAD and TPCONTROL have been developed. PTLOAD optimizes the load arrangement within the furnace, and TPCONTROL proposed a new thermal schedule to reduce cycle time and energy consumption. By comparing the relative cost of possible load arrangement designs, PTLOAD can assist to determine the optimum load arrangement in the furnace for a combined consideration of time, energy and quality. The modified thermal cycle and control algorithms adopted in TPCONTROL are effective in reducing cycle time and energy consumption while avoiding overheating. Nevertheless, it should be noted that the amount of saving varies from case to case. 


\section{Section 1: Thermal Prediction}

\subsection{QUENCHCALC}

The QUENCHCALC provides a methodology to estimate the time-temperature data during quenching with the appropriate initial and boundary conditions and to predict the attainable mechanical properties from a specific quench path at selected locations of a part. The initial condition is the temperature distribution in the part. The boundary condition is usually the effective surface heat transfer coefficient. These heat transfer coefficients are experimentally determined using a variety of quench probes and instrumented parts in a wide variety of polymer solutions, water, and mineral oils. The effective heat transfer coefficient is collected as a function of temperature, type of quenching media, surface condition, part geometry, and part orientation. The data are stored in a database, called QuenchPAD. For a known quench path, the kinetics parameters for precipitation of a secondary phase during quenching can be developed using the quench factor analysis. The Quench Factor can be used to predict the mechanical properties of the part at the selected locations. More basic phase transformation theory has been used to predict the microstructural evolution of the alloy in terms of precipitate nucleation and growth during quenching as well as after the aging heat treatments.

\subsubsection{The Effects of Polymer Concentration and Agitation on the Quench Performance of Polymer Quenchant Aqua-Quench 260}

\subsubsection{Materials and Experimental Apparatus}

The mechanical properties of cast aluminum alloy A356 are very attractive for many applications in military and aircraft industries since the silicon, as the major alloying element, can offer excellent castability, good corrosion resistance, and machinability. The presence of small amount of magnesium makes the alloy heat treatable. The mechanical properties of the alloy can be greatly improved by heat treatment (T4 or T6). Chemical modification dramatically alters the morphology of eutectic silicon particles and provides a wide range of properties. Cast aluminum alloy A356 with the chemical composition in Table 1.3-1 is selected for the present investigation. The alloy in this study is modified with $0.02 \%$ strontium. It is reported that the addition of 


\section{Section 1: Thermal Prediction}

$0.008 \%$ strontium is sufficient to change an acicular eutectic to a finely dispersed fibrous eutectic for non-modified A356 alloy.

Table 1.3-1. Chemical composition of cast aluminum alloy A356 (wt\%)

\begin{tabular}{ccccccccc}
\hline $\mathbf{S i}$ & $\mathbf{M g}$ & $\mathbf{C u}$ & $\mathbf{M n}$ & $\mathbf{F e}$ & $\mathbf{Z n}$ & $\mathbf{T i}$ & $\mathbf{S r}$ & $\mathbf{A l}$ \\
\hline 7.20 & 0.35 & 0.01 & 0.0026 & 0.125 & 0.01 & 0.13 & 0.02 & Balance \\
\hline
\end{tabular}

Among the major casting processes, permanent mold casting can provide better mechanical properties, smoother cast surface, less tendency for entrapped gas, and finer dendrite arm spacing and grain structure. Aluminum A356 cylindrical bars, $1 "(2.54 \mathrm{~cm})$ in diameter and 8 " $(20.32 \mathrm{~cm})$ in length, were cast in the WPI Metal Processing Institute Advanced Casting Laboratory. The bars were cast in a permanent cast iron mold. The casting mold was preheated to $427^{\circ} \mathrm{C}\left(800^{\circ} \mathrm{F}\right)$ in a GECO BHT30 furnace. About $40 \mathrm{lbs}$ of A356 knuckles were melted in a MELLEN CC12 resistance furnace and cast into the pre-heated cast iron mold. Prior to casting, the melt was degassed using Argon gas for about 90 minutes. A rotary impeller was used to agitate the melt. The melt pouring temperature was kept constant at $800^{\circ} \mathrm{C}\left(1472^{\circ} \mathrm{F}\right)$. Cylindrical specimens, 1" in diameter and 4" in length as shown in Figure 1.3-1, were fabricated from the cast bars and used in this study. As-cast surface was used in the quenching.

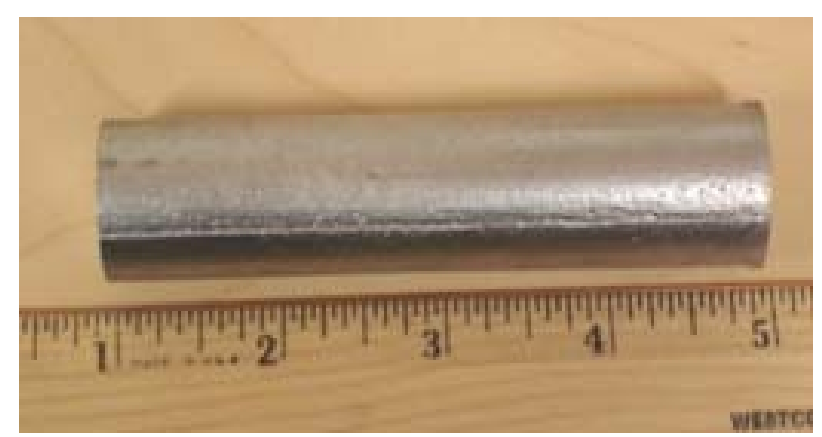

Figure 1.3-1. A cylindrical specimen of cast aluminum alloy A356 


\section{Section 1: Thermal Prediction}

CHTE quench-agitation system in Figure 1.3-2 was used in this investigation, which consisted of a MELLEN tubular furnace MA\#100038 for heating the specimens, agitation system, data acquisition system, and connecting rod-coupling-probe assembly. A U-shaped tube in the quench tank was used to direct the flow. An impeller, for agitation purpose, was introduced to the tube from one end; specimens were quenched into the other end when they were ready. Different agitation levels were obtained by adjusting the rotating speed of the impeller.

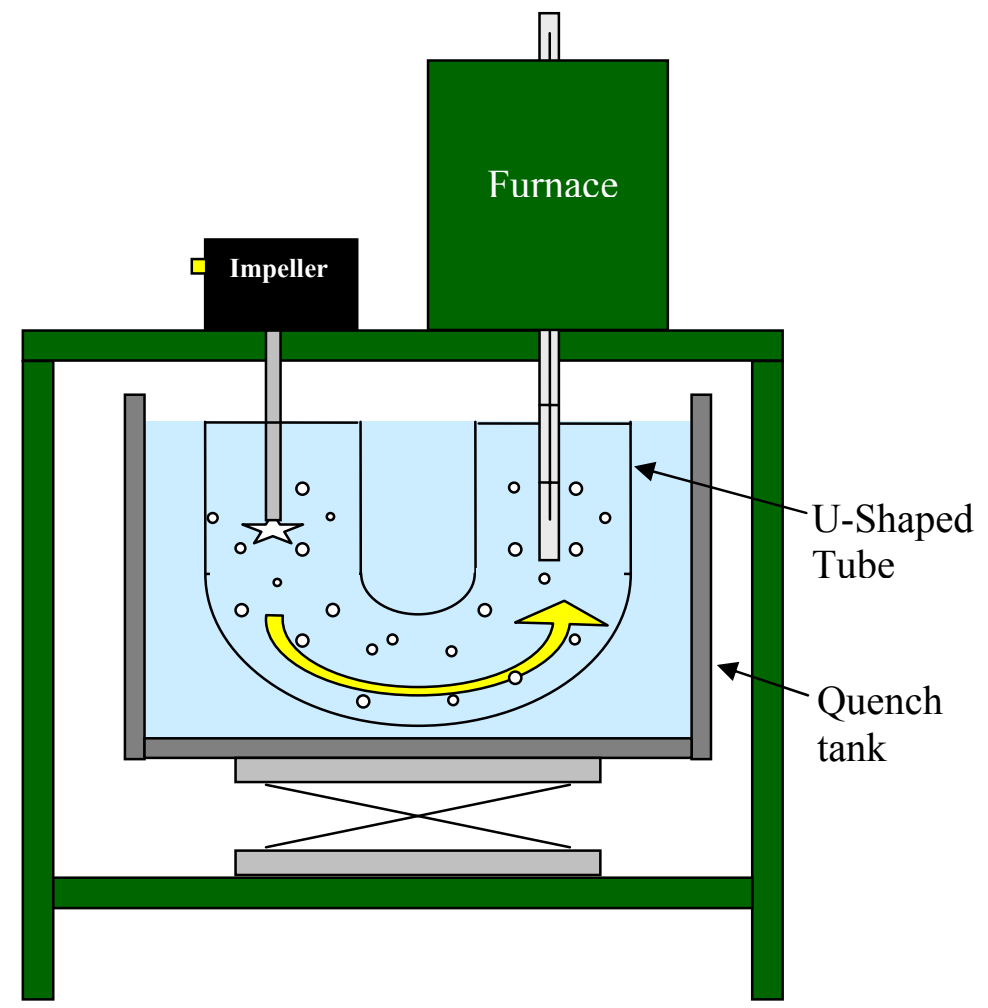

Figure 1.3-2. CHTE quench system

\subsubsection{Taguchi Design of Experiments}

Aqueous solutions of polymer quenchants have the advantage of providing more uniform quench over water by extending the vapor blanket stage to a lower temperature. In terms of polymer quench, there are two important parameters, which are the polymer concentration and the agitation applied to the quenchant. Quantitative measurement of the contribution from each process parameter to the heat extraction rate is a necessity for understanding the quenching 


\section{Section 1: Thermal Prediction}

process. Taguchi technique[3] is employed for designing the test matrix to study these process parameters.

The velocity of a fluid attainable with the current agitation setup (impeller and U-shaped tube) was measured with a Turbo meter near one end of the U-shaped tube. In Figure 1.3-3 it shows that the velocity increases with the speed of impeller and remains constant after certain agitation level is reached. Beyond this level more turbulent flow is observed. Three agitation levels, labeled as low $(0.5 \mathrm{ft} / \mathrm{sec})$, medium $(1.5 \mathrm{ft} / \mathrm{sec})$, and high $(2.5 \mathrm{ft} / \mathrm{sec})$ in Figure 1.3-3, were selected to be the input agitation levels in the Taguchi matrix. Another parameter of concern in Taguchi matrix is the polymer concentration. $10 \%, 20 \%$, and $30 \%$ of polymer solution were chosen to be the levels of interest according to the recommendation from the manufacturer.

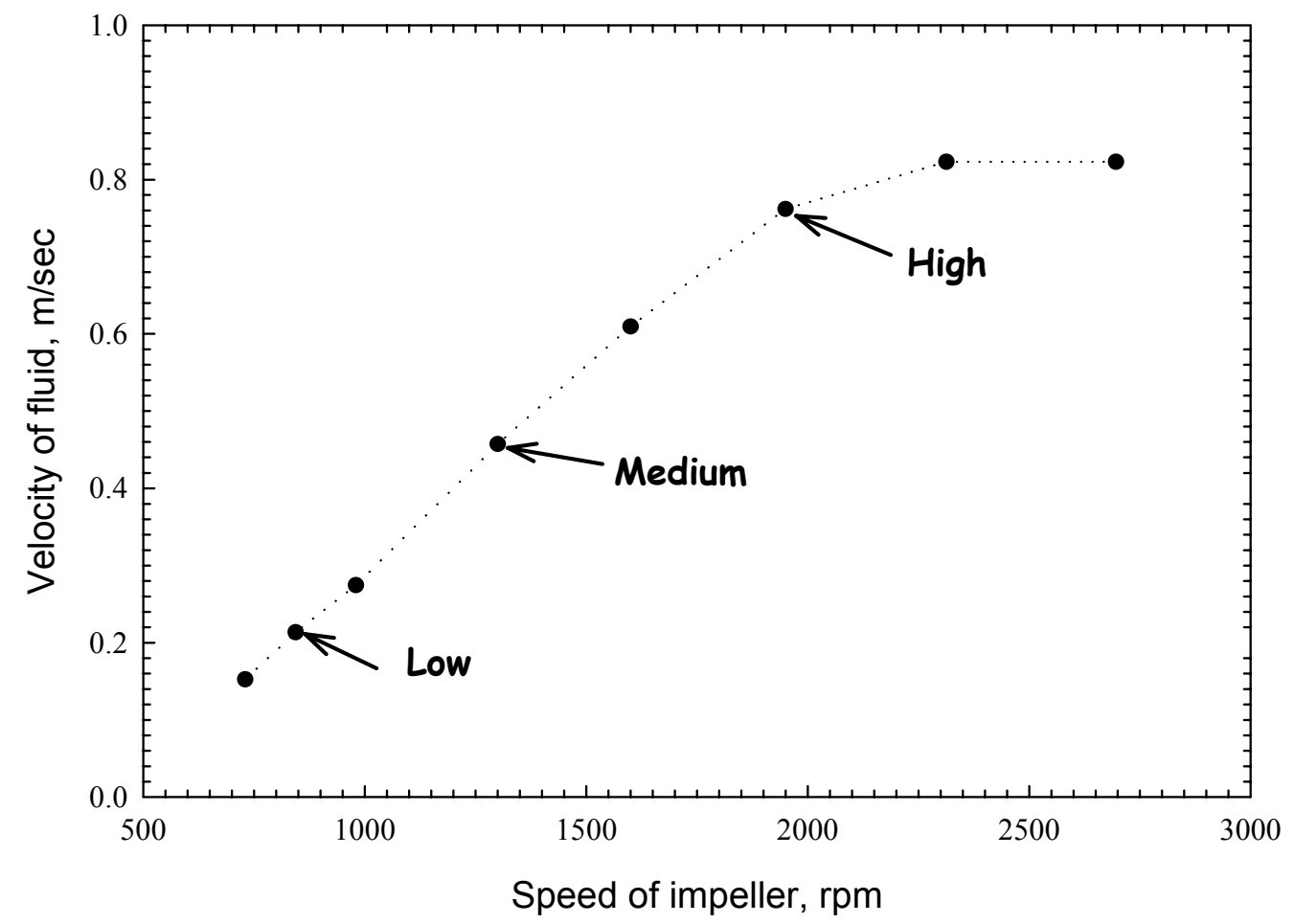

Figure 1.3-3. The variation of fluid velocity with the speed of impeller 


\section{Section 1: Thermal Prediction}

Table 1.3-2. Process parameters and the levels

\begin{tabular}{ccccc}
\hline & \multirow{2}{*}{ Factors } & \multicolumn{3}{c}{ Level } \\
\cline { 3 - 5 } & & $\mathbf{1}$ & $\mathbf{2}$ & $\mathbf{3}$ \\
\hline A & Polymer concentration & $10 \%$ & $20 \%$ & $30 \%$ \\
& Agitation, $\mathbf{r p m}$ & $730(\mathrm{Low})$ & 1300 (Medium) & 1950 (High) \\
B & Agitation, ft/sec & 0.5 & 1.5 & 2.5 \\
\hline
\end{tabular}

Two variables, each at three levels, were used in the matrix to study the heat transfer performance of cast aluminum alloy A356 in polymer solutions. Three-Level L9 orthogonal arrays were chosen to be the layout of DOE matrix. Table 1.3-2 summarizes the process parameters and the selected levels. Table 1.3-3shows the Taguchi L9 layout. "1, 2, 3" in Table 1.3-3stands for the variable level in Table 1.3-2. The percentage of effects from each variable and their interaction was analyzed by analysis of variance (ANOVA).

Table 1.3-3. Taguchi L9 Layout (Three-Level orthogonal arrays)

\begin{tabular}{|c|c|c|c|c|}
\hline \multirow[b]{2}{*}{ Trial No. } & \multicolumn{4}{|c|}{ Column No. } \\
\hline & 1-A (Concentration) & 2-B (Agitation) & $3-\mathbf{A} \times \mathbf{B}$ & 4 \\
\hline 1 & 1 & 1 & & \\
\hline 2 & 1 & 2 & & \\
\hline 3 & 1 & 3 & & \\
\hline 4 & 2 & 1 & & \\
\hline 5 & 2 & 2 & & \\
\hline 6 & 2 & 3 & & \\
\hline 7 & 3 & 1 & & \\
\hline 8 & 3 & 2 & & \\
\hline 9 & 3 & 3 & & \\
\hline
\end{tabular}

\subsubsection{Heat transfer}

Specimens were solutionized at $540^{\circ} \mathrm{C}$ for 4 hours in a MELLEN tubular furnace MA\#100038, quenched in water, polymer solution, and air at room temperature. The bars were then sliced into smaller disks and the individual pieces were aged at $165^{\circ} \mathrm{C}$ for $0,2,4,6,8,10,12$ and 14 hours to study the aging kinetics. The time-temperature data were collected during the quenching 


\section{Section 1: Thermal Prediction}

process using Labview VI 6.1. K-type thermocouples were placed in the geometric center of the specimens for this purpose. The collected data were smoothed by a running average method, an embedded algorithm in SigmaPlot (data analysis software). The first derivative of temperature in terms of time, called cooling rate, was taken to reveal the quenching stages and to compare the quench sensitivity of the alloy under different test conditions.

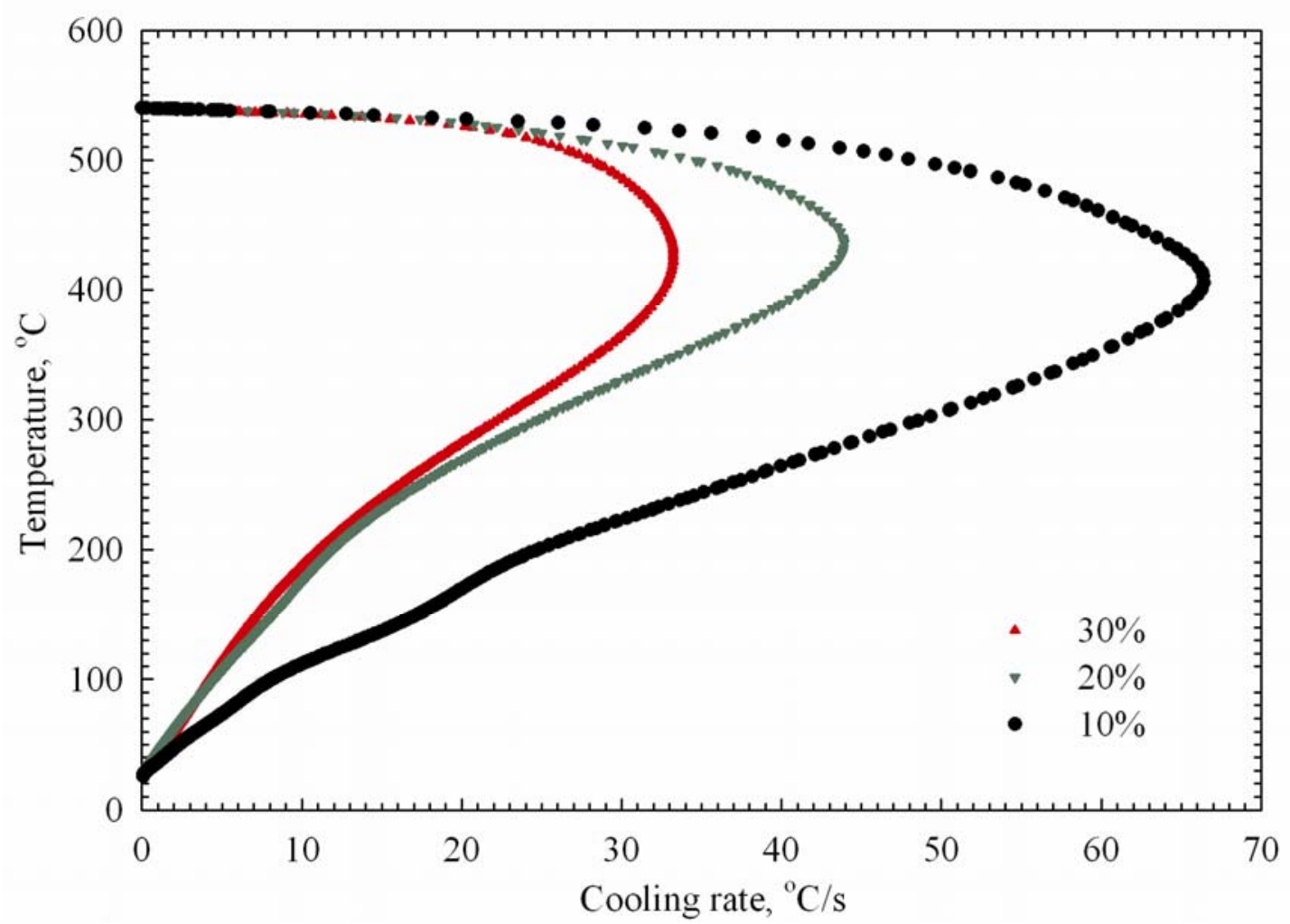

Figure 1.3-4. Cooling rate curves of cast aluminum alloy A356 quenched in different concentrations of polymer solution with medium level of agitation (1300rpm)

As shown in Table 1.3-3, nine tests of combination, three levels of concentration and three levels of agitation, were designed. Each condition was repeated for 3 times to produce the repeatability of the results. Figures 1.3-4 and 1.3-5 respectively shows the cooling rate curves of cast aluminum alloy A356 corresponding to different polymer concentrations and agitation levels. From Figure 1.3-4, the dramatic increase in the cooling rate with the increase in polymer 


\section{Section 1: Thermal Prediction}

concentration in the range of $10 \%$ to $30 \%$ is observed. The maximum cooling rate varies from $30^{\circ} \mathrm{C} / \mathrm{sec}$ to $70^{\circ} \mathrm{C} / \mathrm{sec}$ with the decrease in polymer concentration by $20 \%$. If the individual quenching stage is examined, no much difference is seen in convection stage, but large variations are observed in the partial film boiling and nucleate boiling regimes.

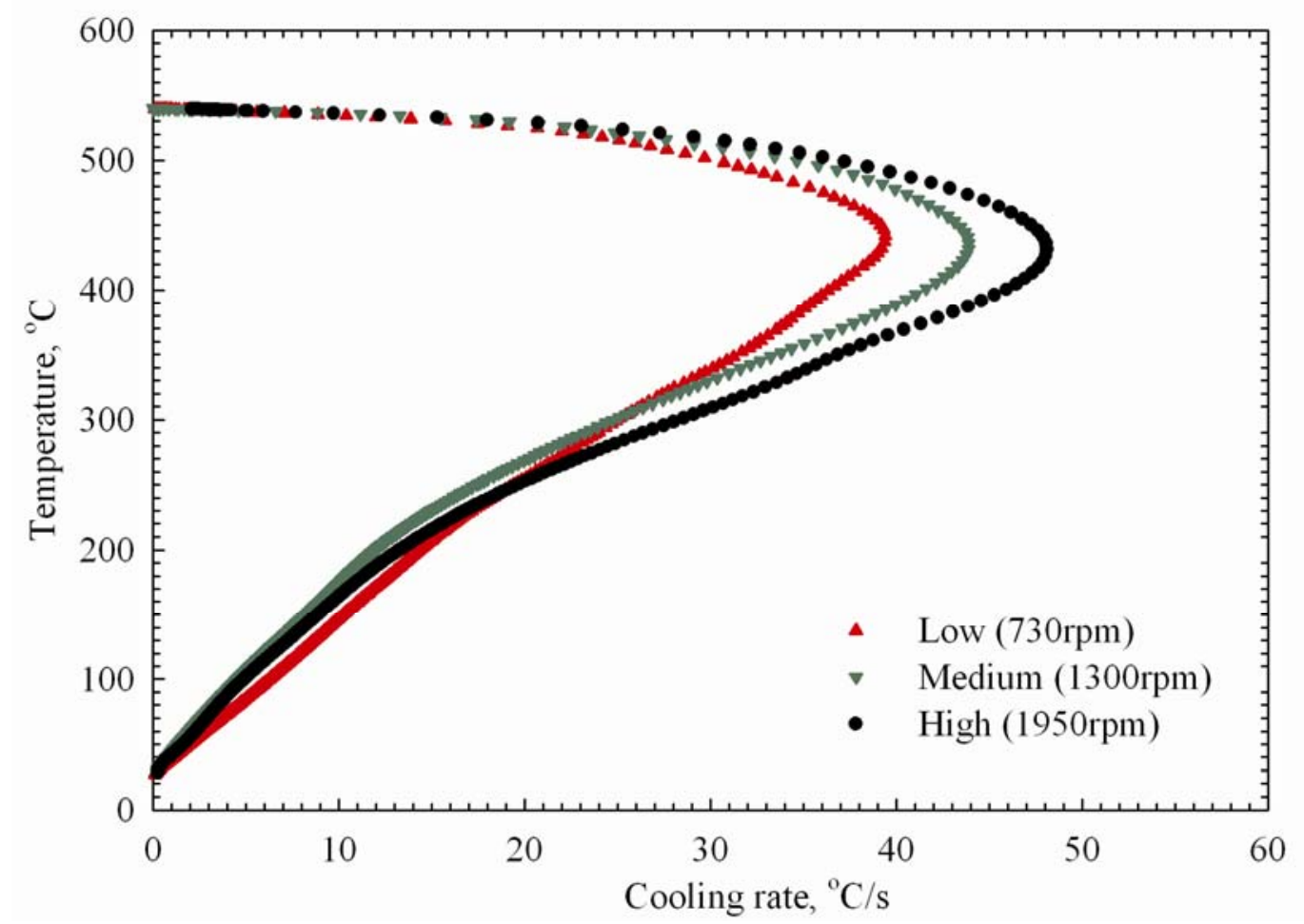

Figure 1.3-5. Cooling rate curves of cast aluminum alloy A356 quenched in 20\% Aqua 260 at different agitation levels

Cooling rate curves of cast aluminum alloy A356 quenched in 20\% Aqua 260 polymer solution at different agitation levels are presented in Figure 1.3-5, slight increase in cooling rate is seen when the agitation level increases. Agitation does enhance the heat transfer although no significant increase is seen from agitation as seen from concentration.

\subsubsection{ANOVA analysis}




\section{Section 1: Thermal Prediction}

The average cooling rate between $460^{\circ} \mathrm{C}$ and $280^{\circ} \mathrm{C}$ was chosen as the response variable for quantifying the effects of concentration and agitation. The selection of the temperature range is based on the CCT diagram of cast aluminum alloy A356 generated using JmatPro software and some reference data in the literature. The analysis of the experimental results is focused on maximizing the average cooling rate between $460^{\circ} \mathrm{C}$ and $280^{\circ} \mathrm{C}$ since this temperature range is critical for the precipitation of secondary phase, $\mathrm{Mg}_{2} \mathrm{Si}$, during the quenching of cast aluminum alloy A356. The variations of average cooling rate with the concentration and agitation are given in Figure 1.3-6. The plot on the left side shows that average cooling rate slightly changes with agitation level for all three concentrations, while the plot on the right side reveals a dramatic drop in the average cooling rate with the increase in concentration.
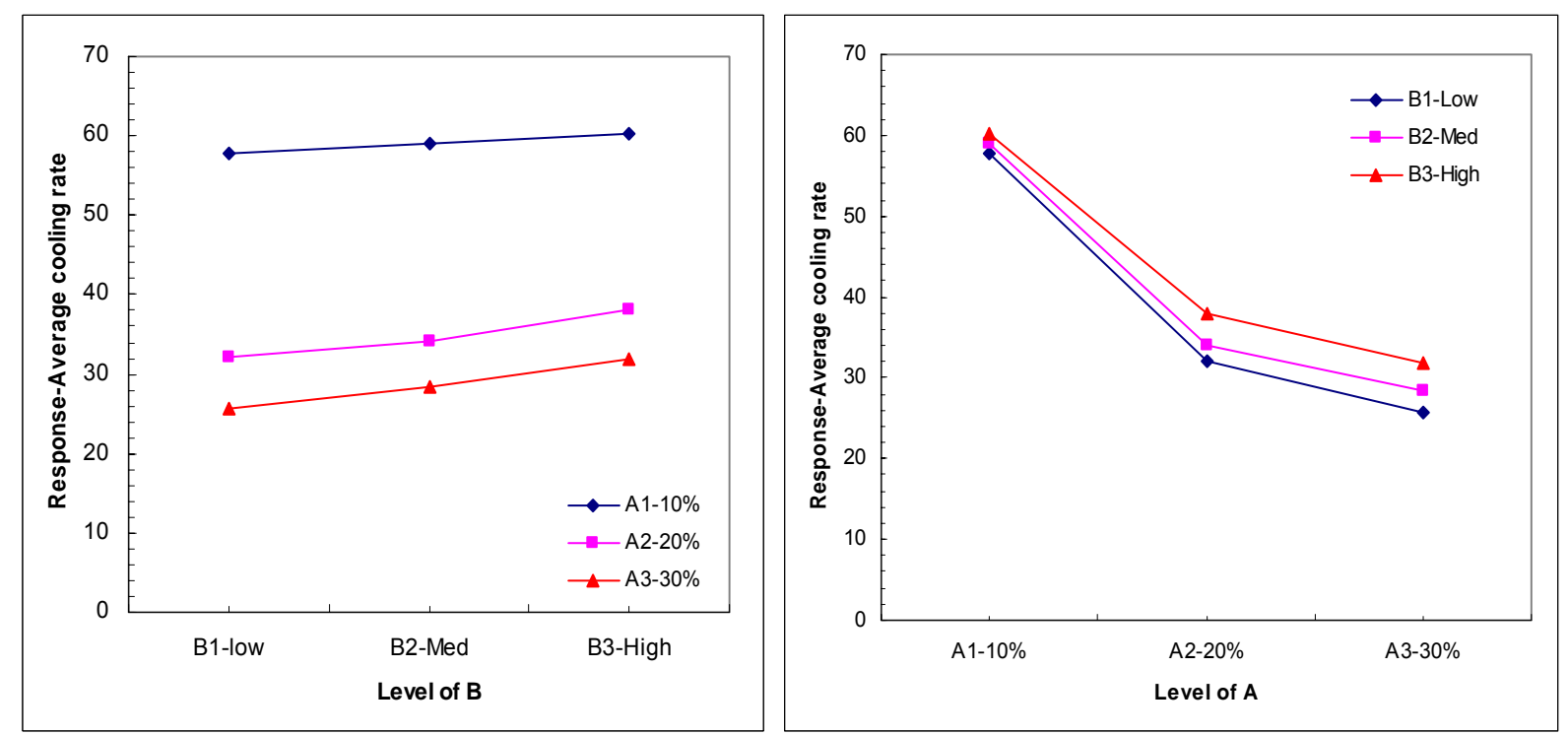

Figure 1.3-6. Variations of average cooling rate of cast aluminum alloy A356 with polymer concentration and tank agitation

The goal of designing the experiments using the Taguchi technique is to optimize the experimental settings or process parameters for a multivariable process with least experimental efforts and to evaluate the experimental results with analysis of variance (ANOVA). In this study ANOVA was performed on the quenching data to quantitatively evaluate the effect from each 


\section{Section 1: Thermal Prediction}

process parameter and their interaction. Table 1.3-4 shows the results from this analysis. The percentage contribution reveals the relative effect from each variable or their interaction. From Table 1.3-4, the process parameter that affects the variation of average cooling rate most is the polymer concentration. The percentage of contribution from polymer concentration is $97 \%$. The influences that the agitation and the interaction between concentration and agitation have on the average cooling rate are relatively insignificant. The same conclusion can also be drawn from Figure 1.3-6.

Table 1.3-4. ANOVA analysis table for average cooling rate

\begin{tabular}{lcccc}
\hline Factors & Freedom & $\begin{array}{c}\text { Sum of } \\
\text { squares }\end{array}$ & Variance & $\begin{array}{c}\text { Percentage of total } \\
\text { effect }\end{array}$ \\
\hline Factor A-concentration & 2 & 1552.20 & 776.10 & $97.54 \%$ \\
Factor B-Agitation & 2 & 36.93 & 18.46 & $2.32 \%$ \\
Factor A $\times$ B & 4 & 4.30 & 1.08 & $0.14 \%$ \\
All other /error & 1 & 0.00 & & \\
& & & & $100.00 \%$ \\
\hline \multicolumn{1}{c}{ Total of sum of squares } & 9 & 1593.43 & 795.64 & \\
\hline
\end{tabular}

\subsubsection{CFD simulation}

The fluid field upon agitation was simulated using a numerical method called computational fluid dynamics (CFD) to visualize the magnitude and direction of the flow in the quench tank. CFD utilizes a computer model to solve the complex fluid flow that is often too difficult to solve with experimental or analytical techniques. There are many programs that use CFD to model fluid flow, in this study one specific program called Fluent [4] was used. The physical model and meshing of the quench tank were generated in Gambit and then imported into Fluent. The physical properties of fluid and materials and interfaces and boundary conditions were defined before the case was initiated. The program was run for a specified number of iterations. The iterative process was not completed until the convergence criterion was met. A residual plot was 


\section{Section 1: Thermal Prediction}

generated in Fluent to monitor the convergence of the case. In this study, two cases with different combinations of concentration and agitation were simulated. The test matrix is shown in Table 1.3-5.

Table 1.3-5. Parameters used in CFD simulation

\begin{tabular}{|c|c|c|}
\hline & Concentration (\%) & Agitation (rpm) \\
\hline \multirow{2}{*}{ Case 1 } & $10 \%$ & 1300 \\
& $30 \%$ & 1300 \\
\multirow{2}{*}{ Case 2 } & $20 \%$ & 730 \\
\cline { 2 - 3 } & $20 \%$ & 1950 \\
\hline
\end{tabular}

As shown in Table 1.3-5, two cases were selected for CFD simulation to visualize the velocity distribution in the U-shaped tube and quench tank. The simulation was performed with the presence of impeller in the tube but without the quench probe. The viscosity and density of polymer solution was included in the boundary conditions of the model. Figure 1.3-7 gives the contour plot of velocity magnitude for two different levels of polymer concentration, $10 \%$ and $30 \%$, upon the same agitation, 1300rpm. The color bar on the left side of the plots indicates the velocity magnitude. Red color stands for the higher velocity and blue color represents lower velocity. The contour plot reveals the velocity distribution in the U-shaped tube. The same flow pattern is observed in Figure 1.3-7 for two different concentration levels upon the same agitation. Although a small dead zone is seen at the horizontal part of the tube, from the contour plot it can be seen the U-shaped tube does help direct the flow. Compared with the H-baffle used in the early stage of studying the agitation, U-shaped tube is more efficient. During the quenching tests, the quench probe was quenched into the same depth from the top surface of the U-shaped tube with the impeller on the other end. The magnitude of velocity at the location where the probe was quenched was extracted from the simulation data and listed in Table 1.3-6. Under the same agitation, velocity doesn't vary much with the variation in polymer concentration. However, from Figure 1.3-8 and the data in Table 1.3-6, if the polymer concentration is constant, increasing the agitation does increase the velocity to a great extent. 


\section{Section 1: Thermal Prediction}

Table 1.3-6. Velocity magnitude from the Fluent simulation

\begin{tabular}{|c|c|c|}
\hline Polymer Concentration (\%) & Agitation (rpm) & Velocity (m/s) \\
\hline $\mathbf{1 0} \%$ & 1300rpm & 1.0 \\
$\mathbf{3 0} \%$ & 1300rpm & 1.0 \\
$\mathbf{2 0} \%$ & 730rpm & 0.75 \\
\hline $\mathbf{2 0} \%$ & 1950rpm & 2.5 \\
\hline
\end{tabular}




\section{Section 1: Thermal Prediction}

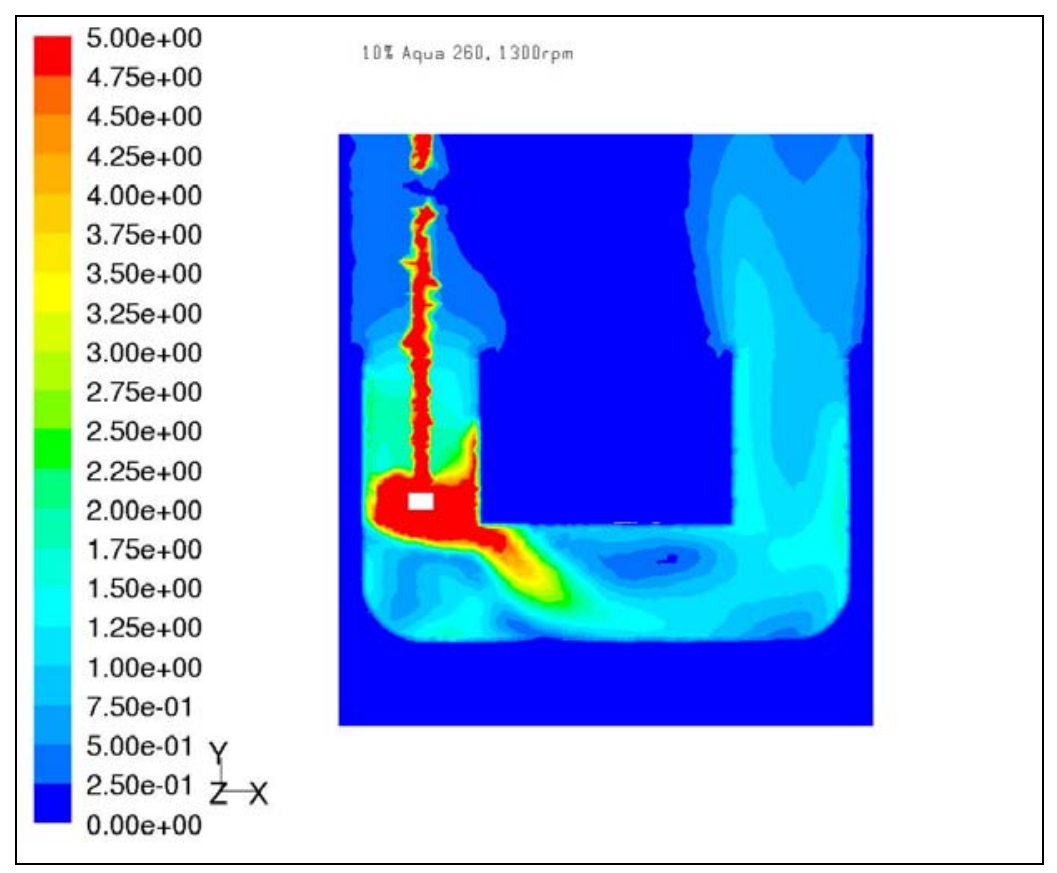

(a)

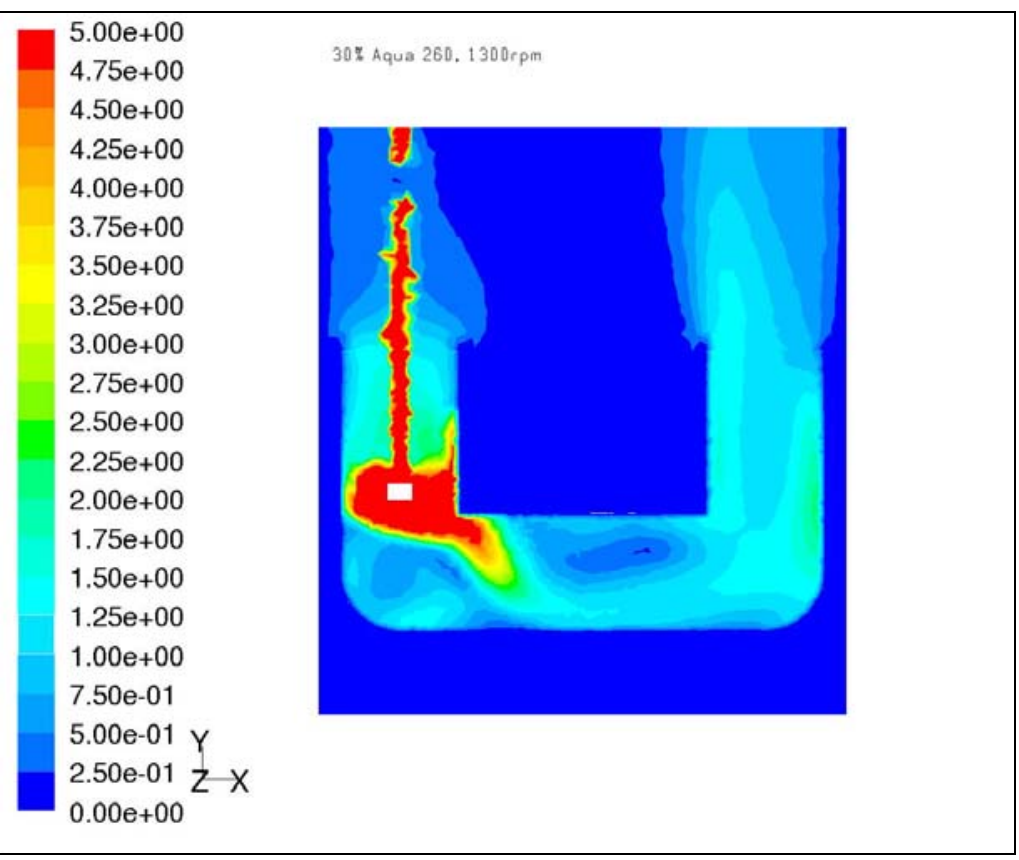

(b)

Figure 1.3-7. Contour plot of velocity field simulated in Fluent for case (a) 10\% Aqua 260 (b) $30 \%$ Aqua 260 upon 1300rpm agitation 


\section{Section 1: Thermal Prediction}

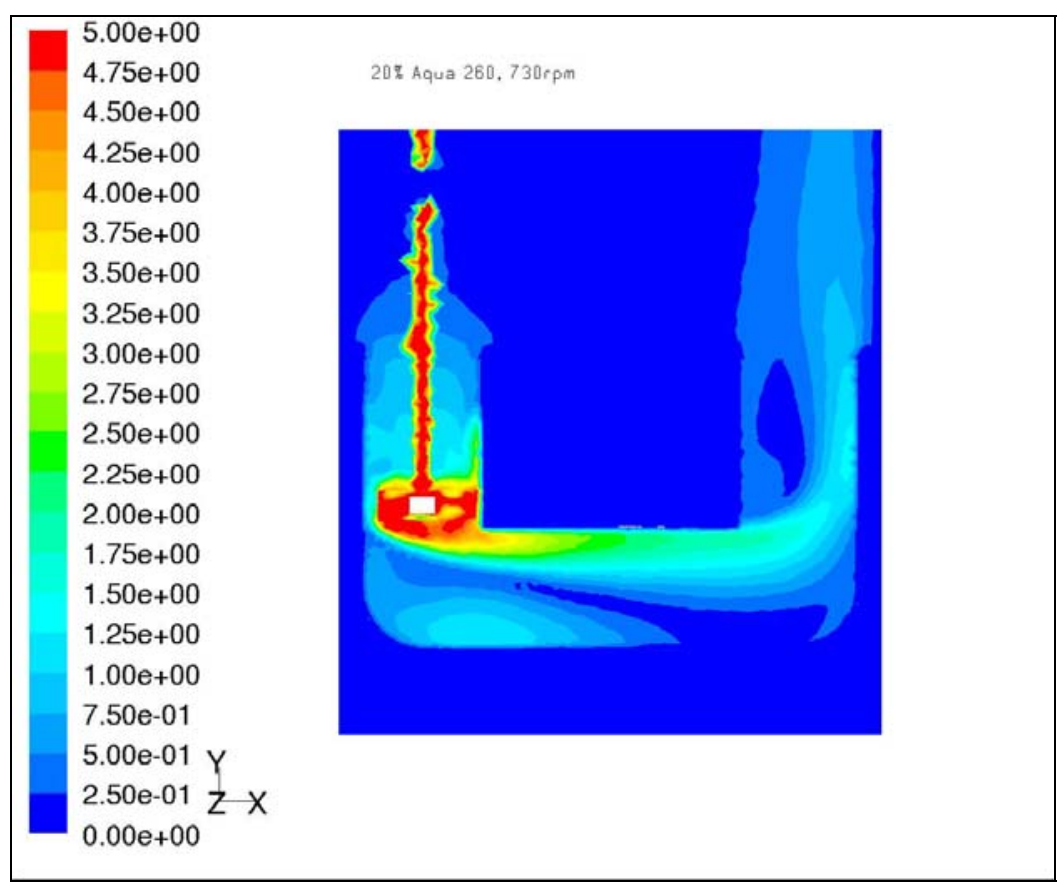

(a)

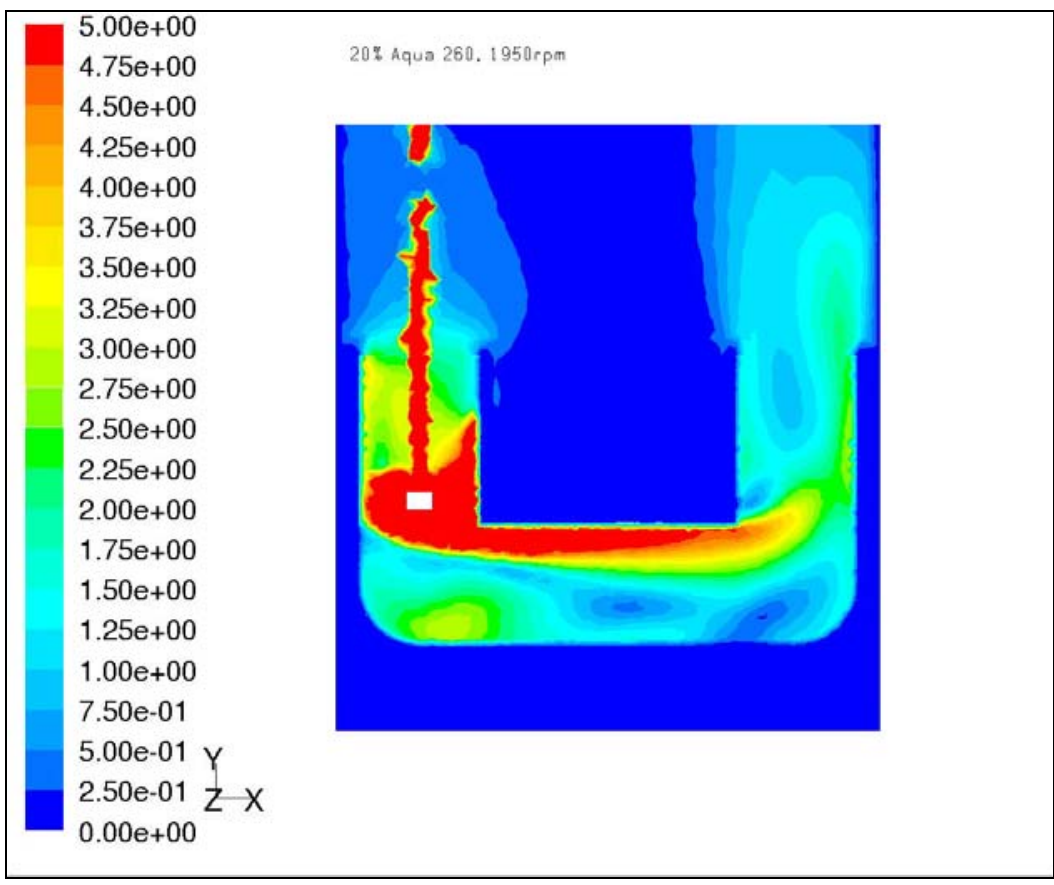

(b)

Figure 1.3-8. Contour plot of velocity field in $20 \%$ Aqua 260 polymer solution simulated in Fluent for an agitation level of (a) 730rpm (b) 1950rpm 


\section{Section 1: Thermal Prediction}

The velocity was measured near one end of the U-shaped tube with a Turbo meter. The fluid used in the measurement is water at room temperature. The simulated velocity with polymer solution at the same location was obtained from the velocity contour plot. The results are given in Table 1.3-7. Simulated and measured velocities are in the same range with the simulated one slightly higher.

Table 1.3-7. Simulated and measured velocity at one end of the U-shaped tube

\begin{tabular}{|c|c|c|c|}
\hline $\begin{array}{c}\text { Polymer Concentration } \\
(\mathbf{\%})\end{array}$ & $\begin{array}{c}\text { Agitation } \\
(\mathbf{r p m})\end{array}$ & $\begin{array}{c}\text { Measured velocity with } \\
\text { water } \mathbf{( m / \mathbf { s } )}\end{array}$ & $\begin{array}{c}\text { Simulated velocity } \\
(\mathbf{m} / \mathbf{s})\end{array}$ \\
\hline $10 \%$ & 1300 & & 0.75 \\
\hline $30 \%$ & 1300 & 0.5 & $0.75 \sim 1.0$ \\
\hline $20 \%$ & 730 & 0.2 & $0.25 \sim 0.5$ \\
\hline $20 \%$ & 1950 & 0.8 & $1.0 \sim 1.35$ \\
\hline
\end{tabular}

\subsubsection{Hardness measurements}

The polymer concentration was determined to be the dominating process parameter from the above analysis of variance. The aging kinetics of cast aluminum alloy A356 was investigated after the specimens were solutionized and quenched in different concentrations of Aqua 260 polymer solution. The detailed test matrix is shown in Table 1.3-8. The tank agitation used in this study is $1300 \mathrm{rpm}$ (medium level). After the solutionizing-quenching-aging process, the samples were ground with $\mathrm{SiC}$ papers and polished with alumina down to $0.05 \mu \mathrm{m}$. The micro-Vickers hardness measurements were made on the cross section of the as-aged samples using Shimadu HMV-2000 with a load of $25 \mathrm{gf}$ and a dwell time of $10 \mathrm{~s}$. Ten readings were taken in the $\alpha$-aluminum dendrites for each heat treatment condition; the average was used for comparison purpose. The samples were also quenched in water and air for comparison. 


\section{Section 1: Thermal Prediction}

Table 1.3-8. Test matrix for studying the aging kinetics of cast aluminum A356

\begin{tabular}{|c|c|c|c|c|c|c|c|c|}
\hline Solutionizing temperature & \multicolumn{8}{|c|}{$538^{\circ} \mathrm{C}$} \\
\hline Solutionizing time (hour) & \multicolumn{8}{|c|}{4} \\
\hline Quenching medium & \multicolumn{8}{|c|}{ Water, air, $10 \%, 20 \%$, and $30 \%$ Aqua-quench 260} \\
\hline Aging temperature & \multicolumn{8}{|c|}{$165^{\circ} \mathrm{C}$} \\
\hline Aging time (hour) & 0 & 2 & 4 & 6 & 8 & 10 & 12 & 14 \\
\hline
\end{tabular}

Figure 1.3-9 showed the variation of micro-hardness of cast aluminum alloy A356 with aging times after the samples were quenched in different concentrations of aqueous solution of Aqua-quench 260, water, and air. Under all the heat treatment conditions, the micro-hardness increases with the aging time to a peak value and then decreases with a prolonged aging time. This can be explained by the evolution of $\mathrm{Mg}_{2} \mathrm{Si}$ precipitates with the aging time and the interaction between the precipitates and dislocations.

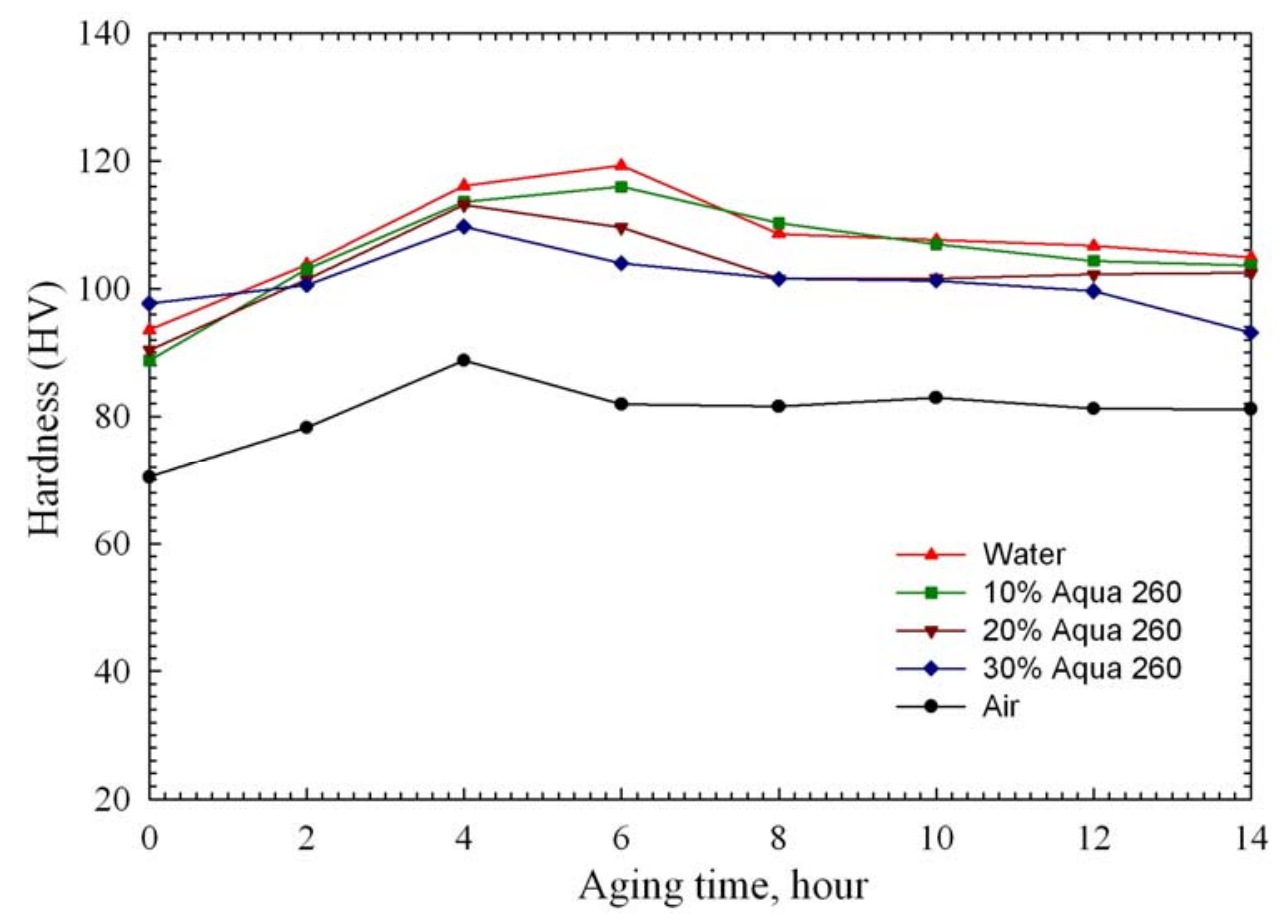

Figure 1.3-9. Vickers hardness of cast aluminum A356 solutionized, quenched in water, polymer solution and air, and aged at $165^{\circ} \mathrm{C}$ for different periods of times 


\section{Section 1: Thermal Prediction}

Water quenched samples show the highest hardness at all the aging times since they are subjected to the fastest cooling and relatively more supersaturated solid solution is retained to the room temperature. For all the polymer quenched samples, the increase in the polymer concentration lowers the attainable hardness if compared with the water quenched sample, as can be seen from Figure 1.3-9. However, the decrease in hardness due to the addition of polymer into water is not substantial. If the benefit, that more uniform quench could be achieved from using polymer solution, is considered, the data in Figure 1.3-9 can provide the support to the advantages of using polymer to reduce the distortion/residual stress without sacrificing the property much. The similar result was reported by D.L.Zhang and L.Zheng in their study of quench sensitivity of cast $\mathrm{Al}-7 \% \mathrm{Si}-0.4 \% \mathrm{Mg}$ alloy [5]. They noticed the peak hardness did not change when the cooling rate decreased from $250^{\circ} \mathrm{C} / \mathrm{s}$ to $110^{\circ} \mathrm{C} / \mathrm{s}$. It can also be seen from Figure 1.3-9 that the samples quenched in water and $10 \%$ polymer solution reaches the peak hardness at 6 hour aging, while the peak hardness is achieved at a shorter aging time (4 hours in this study) for the samples quenched in the $20 \%, 30 \%$ polymer solution, and air. As expected, slow air quench results in the lowest hardness, which is due to the reduction in retained solute concentration from the heterogeneous precipitation during quenching process.

\subsubsection{A Methodology to Predict the Effects of Quench Rates on Mechanical Properties of Cast Aluminum Alloys}

\subsubsection{The Mathematical Model}

Quench factor analysis is a tool for predicting mechanical properties of an alloy with a known quench path and the precipitation kinetics described by Time-Temperature-Property (TTP) curves. TTP curve in Figure 1.3-10 is a graphical representation of the transformation kinetics that influences such properties as hardness or strength [6]. The assumptions behind quench factor analysis are: the precipitation reaction during quenching is additive/isokinetic; and the reduction in strength can be related to the reduction of supersaturation of solid solution during quenching. 


\section{Section 1: Thermal Prediction}

The quench factor is typically calculated from a cooling curve and a $\mathrm{C}_{\mathrm{T}}$ function, an equation that describes the transformation kinetics of an alloy. Evancho and Staley [7] defined the $\mathrm{C}_{\mathrm{T}}$ function as having a form similar to the reciprocal of the nucleation rate equation. This form can be expressed using the following equation [6-9]:

$$
C_{T}=-K_{1} * K_{2} * \operatorname{Exp}\left[\frac{K_{3} * K_{4}{ }^{2}}{R T\left(K_{4}-T\right)^{2}}\right] * \operatorname{Exp}\left[\frac{K_{5}}{R T}\right]
$$

where, $\mathrm{C}_{\mathrm{T}}$ is the critical time required to form a specific percentage of a new phase; $\mathrm{K}_{1}$ is a constant which equals the natural logarithm of the fraction untransformed during quenching (typically $99.5 \%$ : $\operatorname{Ln}(0.995)=-0.00501) ; K_{2}$ is a constant related to the reciprocal of the number of nucleation sites; $K_{3}$ is a constant related to the energy required to form a nucleus; $K_{4}$ is a constant related to the solvus temperature; $\mathrm{K}_{5}$ is a constant related to the activation energy for diffusion; $\mathrm{R}$ is the universal gas constant, $8.3144 \mathrm{~J} / \mathrm{K}^{*} \mathrm{~mol}$; $\mathrm{T}$ is the absolute temperature $(\mathrm{K})$.

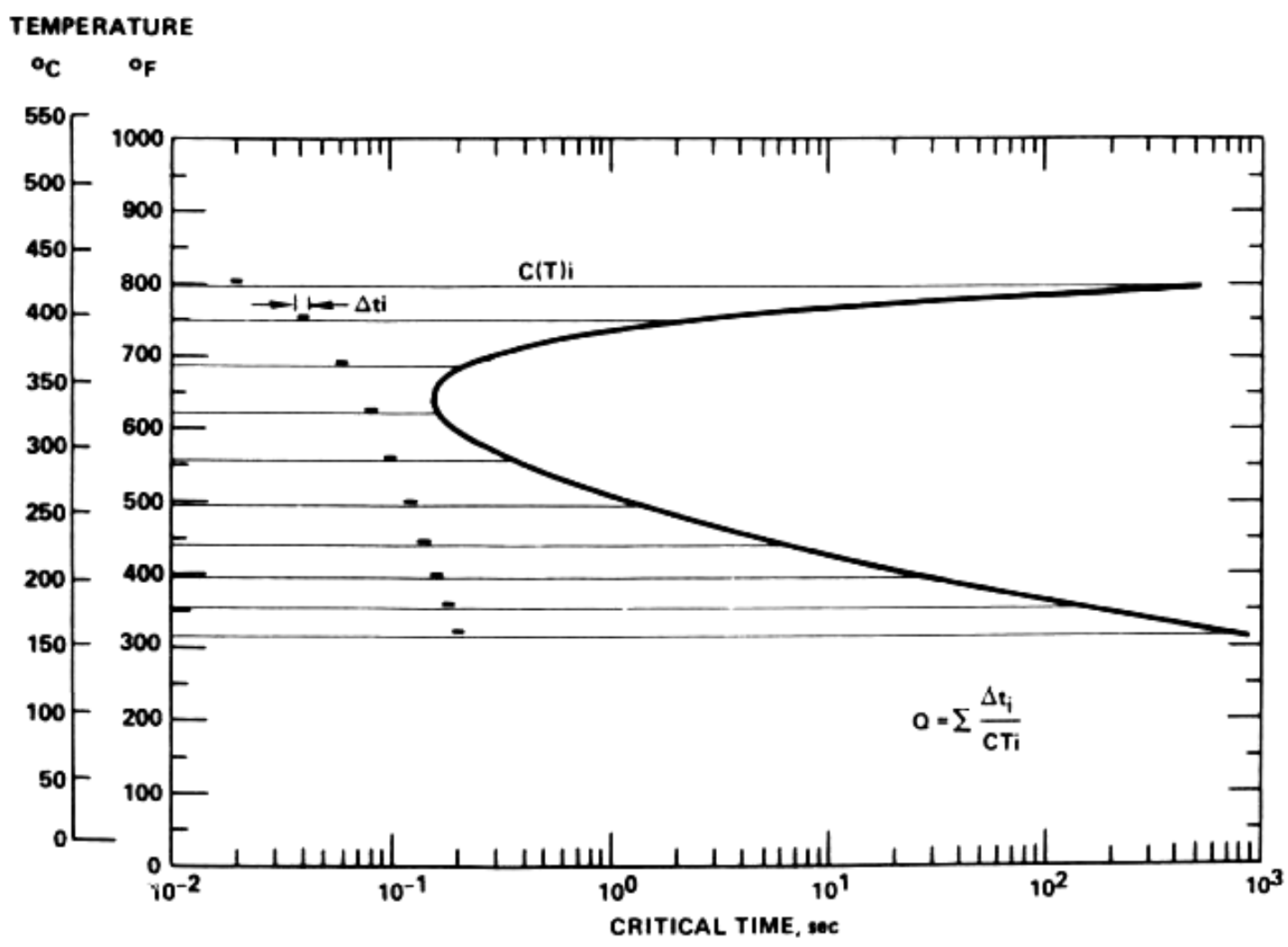

Figure 1.3-10. Schematic illustrations on plot of $\mathrm{C}_{\mathrm{T}}$ function to calculate the quench factor [6]. 


\section{Section 1: Thermal Prediction}

The incremental quench factor, $\mathrm{q}_{\mathrm{f}}$, represents the ratio of the amount of time the alloy is at a particular temperature divided by the time required for a specific amount of transformation [6]. The incremental quench factors can be calculated at each temperature and summed up over the entire transformation range to produce the cumulative quench factor Q $[7,8]$ :

$$
Q=\sum q_{f}=\sum_{T 1}^{T 2} \frac{\Delta t_{i}}{C_{T i}}
$$

where $\mathrm{q}_{\mathrm{f}}$ is the incremental quench factor and $\Delta \mathrm{t}_{\mathrm{i}}$ is the time elapsed at a specific temperature.

With the calculated quench factor Q, the strength can be predicted using the following classical quench factor model $[7,10]$,

$$
\frac{\sigma-\sigma_{\min }}{\sigma_{\max }-\sigma_{\min }}=\exp \left(K_{1} Q\right)^{n}
$$

where $\sigma$ is the strength (In this study, $\sigma$ represents the notation for Meyer hardness); $\sigma_{\max }$ and $\sigma_{\min }$ are the maximum and minimum strength achievable for a specific alloy; $\mathrm{K}_{1}$ is decided above; $\mathrm{n}$ is the Avrami exponent.

Based on the classical quench factor model shown in Equation (1.3-3), improvements have been made to justify the assumptions for quench factor analysis, including the relationship between strength and solute concentration, minimum strength, and Avrami exponent [11]. The assumption of the linear relationship between strength and retained solute concentration was found to contradict the strengthening theory. According to the strengthening theory, Equation (1.3-3) is re-written as the following improved formula [11],

$$
\frac{\sigma-\sigma_{\min }}{\sigma_{\max }-\sigma_{\min }}=\left[\exp \left(K_{1} Q\right)^{n}\right]^{1 / 2}
$$

This statement was justified by the morphology of the secondary phase that could precipitate out from the solid solution during quenching process. 


\section{Section 1: Thermal Prediction}

A variety of mechanical properties have been used for quench factor modeling, including Vickers hardness [9, 11-13], Rockwell hardness [6, 14], electrical conductivity $[12,15]$, yield strength $[10,11,16]$, and tensile strength [12]. Although many successful predictions were made in the literature, the classical quench factor models were established based on the variation of strength with the retained solute concentration and caution has to be taken when any properties other than strength are used in the quench factor modeling unless a linear relationship between the strength and the property exists for the alloy being studied [11]. In this investigation, the Meyer hardness, $\bar{P}$, is the property used in the quench factor modeling, which has an approximately linear relationship with strength. The Meyer hardness is defined as [17],

$$
\bar{P}=\frac{4 L}{\pi d^{2}}
$$

Where $\bar{P}$ is the Meyer hardness, $\mathrm{MPa} ; \mathrm{L}$ is the load, $\mathrm{Kg} ; \mathrm{d}$ is the diameter of indentation, $\mathrm{mm}$.

The relationship between Rockwell hardness and Meyer hardness can be experimentally determined for any specific alloy. For cast aluminum alloy A356, the conversion was established by Tiryakioglu and Campbell using regression analysis of the experimental data [17]. The indentation size, d, is correlated with Rockwell B hardness in the following description [17],

$$
d=1.263-5.270 \times 10^{-3} R H B
$$

Using Equations (1.3-5) and (1.3-6), the Meyer hardness can be calculated from the experimentally measured Rockwell hardness in B scale. The reason of using the Meyer hardness in the quench factor modeling is because it has a linear relationship with strength so the assumptions for quench factor models are still valid in this case.

\subsubsection{Research Methodology}

The research methodology used in this paper for estimating the kinetic parameters of aluminum alloys during quenching, is illustrated in Figure 1.3-11. This methodology starts from preparing an aluminum alloy of interest and casting the Jominy End Quench bars. Based on the ASTM standard A255, the Jominy End Quench tests are performed to experimentally collect 


\section{Section 1: Thermal Prediction}

time-temperature and Rockwell hardness data at selected locations on a bar. The advantage of using Jominy End Quench method for quench factor modeling is that a large range of cooling rates can be obtained with only one quench, which dramatically reduces the experimental efforts that are usually required with any other method. Rockwell hardness is converted to the Meyer hardness using the relationship established by Tiryakioglu and Campbell, as shown in Equations (1.3-5) and (1.3-6) [17]. Multiple linear regression analysis is performed on the experimental data to numerically estimate the kinetic parameters. These kinetic parameters are experimentally verified on a cast engine cylinder head. This methodology requires little experimental effort, has been illustrated for cast aluminum alloy A356, and can be used to experimentally estimate the kinetic parameters during quenching for other heat-treatable aluminum alloys. More detailed procedures of this methodology are in the "results and discussion" section. 


\section{Section 1: Thermal Prediction}

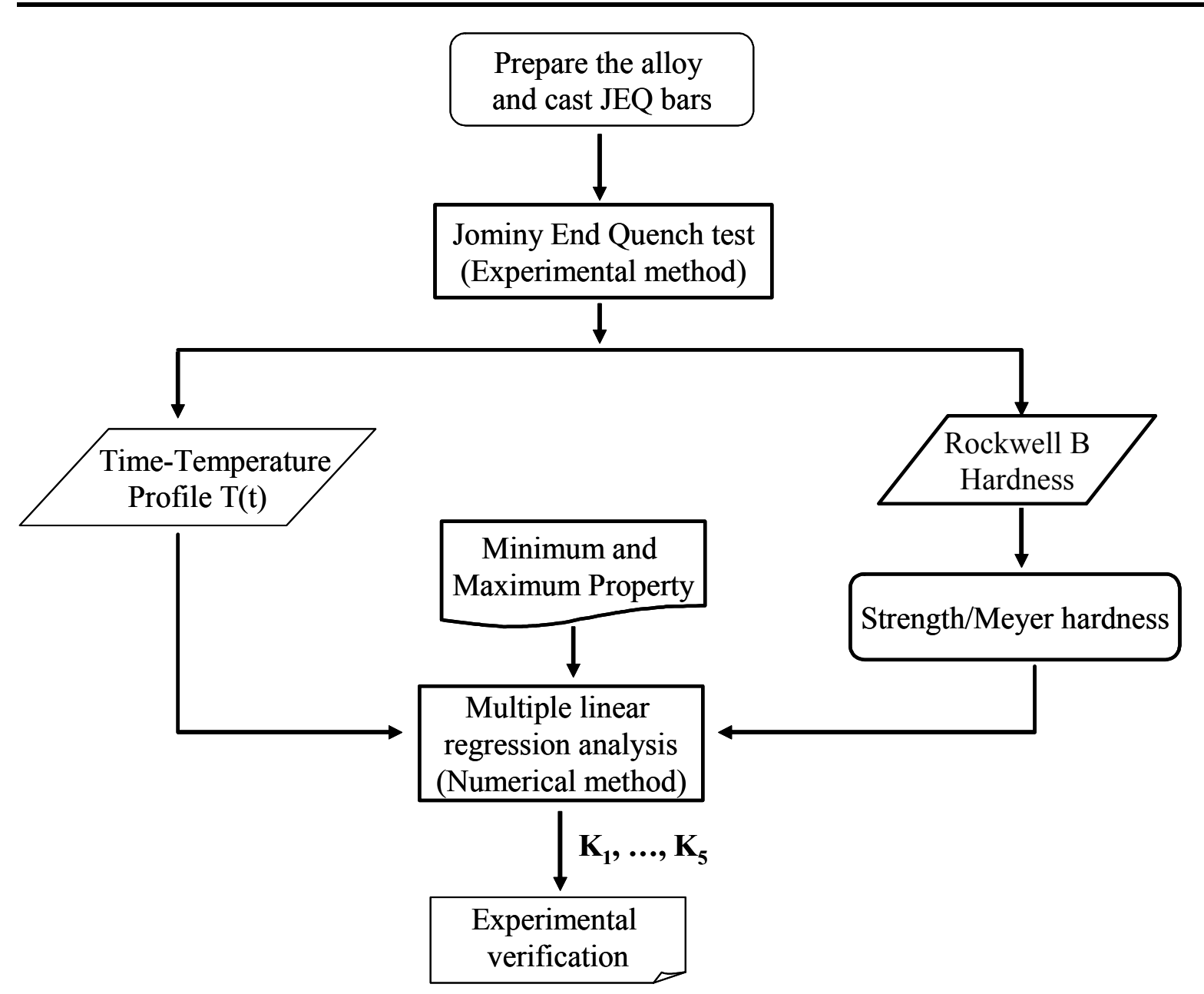

Figure 1.3-11. Overview of the research methodology for quench factor analysis

\subsubsection{Experimental Setup}

The Jominy End Quench apparatus was built according to the standard described in the SAE J406 and ASTM A255 specifications. The schematic of the apparatus is shown in Figure 1.3-12. An orifice with $12.7 \mathrm{~mm}$ in diameter is connected to the waterline through a plastic pipe for quenching. The top plate supports the part in position. According to the standards, the distance between the test specimen and the orifice is $12.7 \mathrm{~mm}$. Since the quenching occurs at one end of a bar, the cooling along an entire Jominy End Quench bar is one-dimensional. 


\section{Section 1: Thermal Prediction}

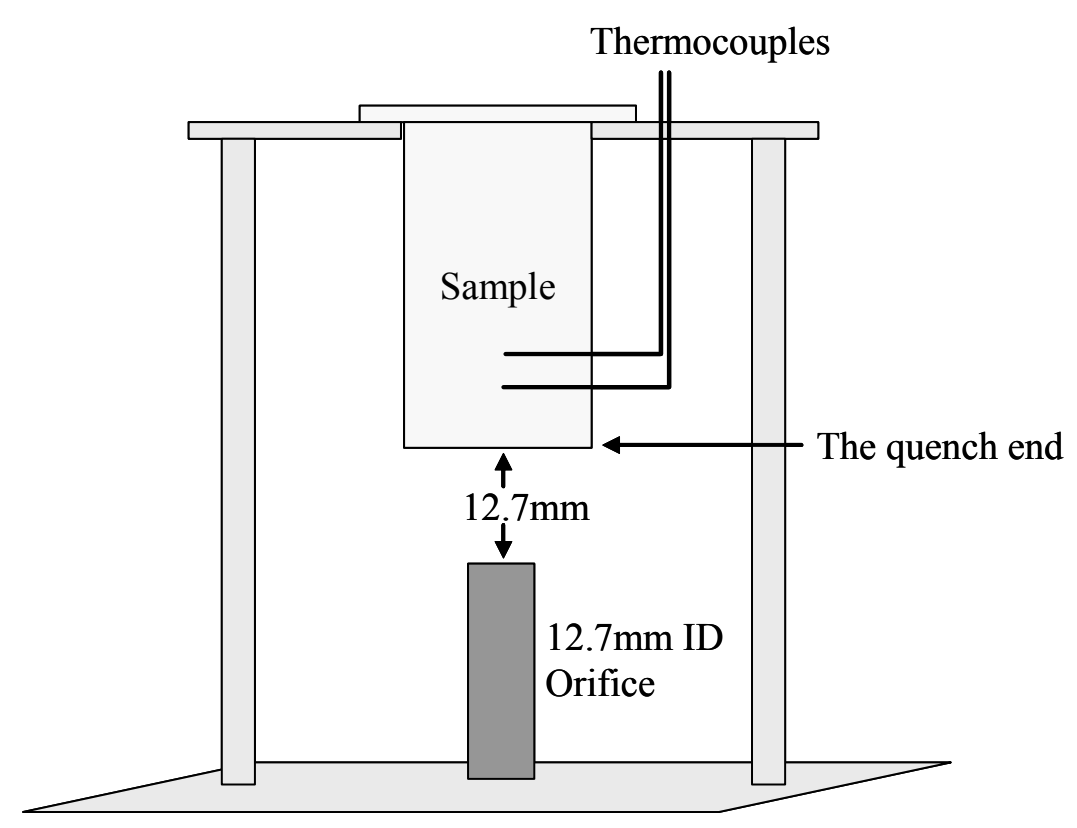

Figure 1.3-12. Schematic of the Jominy End Quench apparatus

\subsubsection{Microstructure of cast aluminum A356}

The microstructure of as-cast and as-solutionized cast aluminum alloy A356 were examined with both scanning electronic microscope and optical microscope. The shape and size of silicon particles reveal the extent of solutionizing. Solutionizing for long periods modifies the morphology of the eutectic silicon. The rounding of silicon particles can effectively improve the ductility and the fatigue properties of the alloy. From Figure 1.3-13, both the spheroidisation of acicular silicon and coarsening of small silicon particles can be observed by comparing the silicon morphology before and after the solutionizing treatment. More spherical particles are seen in the as-solutionized sample that was solutionized at $540^{\circ} \mathrm{C}$ for 4 hours. Average

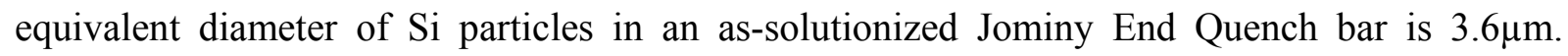
Fe-containing $\pi / \beta$ phases can also be seen on the cell/grain boundaries. These iron-rich phases are detrimental to the materials and require a much longer solutionizing time to be dissolved. In most cases, complete dissolution of iron-containing phases is not observed [18]. 


\section{Section 1: Thermal Prediction}

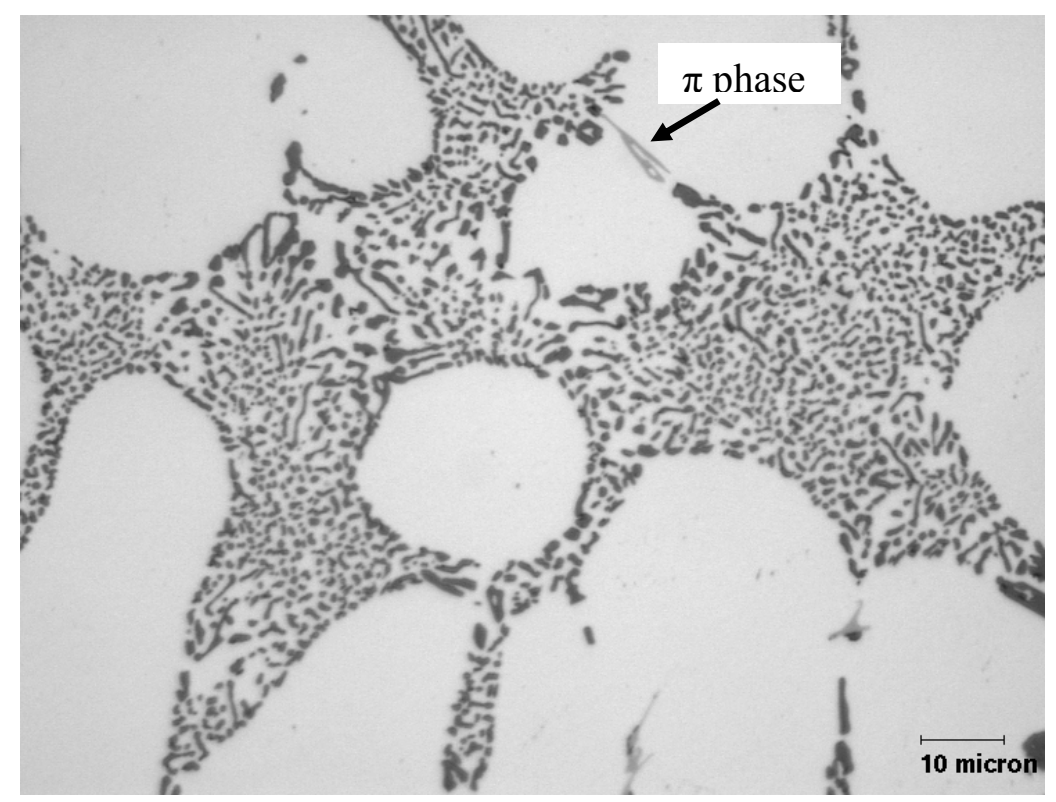

(a) as-cast

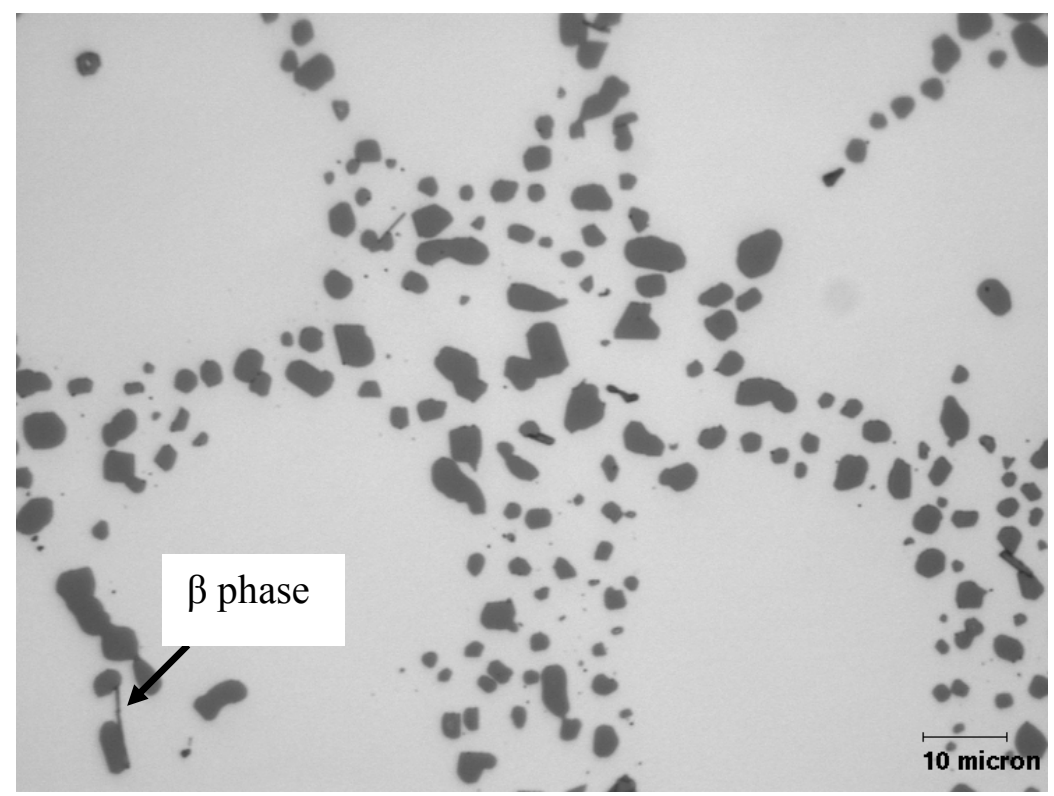

(b) as-solutionized

Figure 1.3-13. Microstructure of (a) as-cast and (b) as-solutionized cast aluminum A356 


\section{Section 1: Thermal Prediction}

To determine the secondary dendrite arm spacing (SDAS) of the aluminum casting in this study and to verify there is no solidification gradient along cast bars, quantitative image analysis was performed at different locations of an as-solutionized Jominy End Quench bar by line intersection method. The magnitude of SDAS is an indication of solidification rate during casting process. SDAS is also an important parameter for estimating the solutionizing time needed for a cast aluminum alloy since it gives the range of the diffusion field for the diffusion of silicon, magnesium, manganese, and other addition elements during the solutionizing treatment in the case of cast aluminum alloy A356. Average size of SDAS for a cast aluminum alloy A356 bar in this study is $27 \mu \mathrm{m}$ and no variation is seen along the entire bar. The results are based on 10 measurements and shown in Figure 1.3-14.

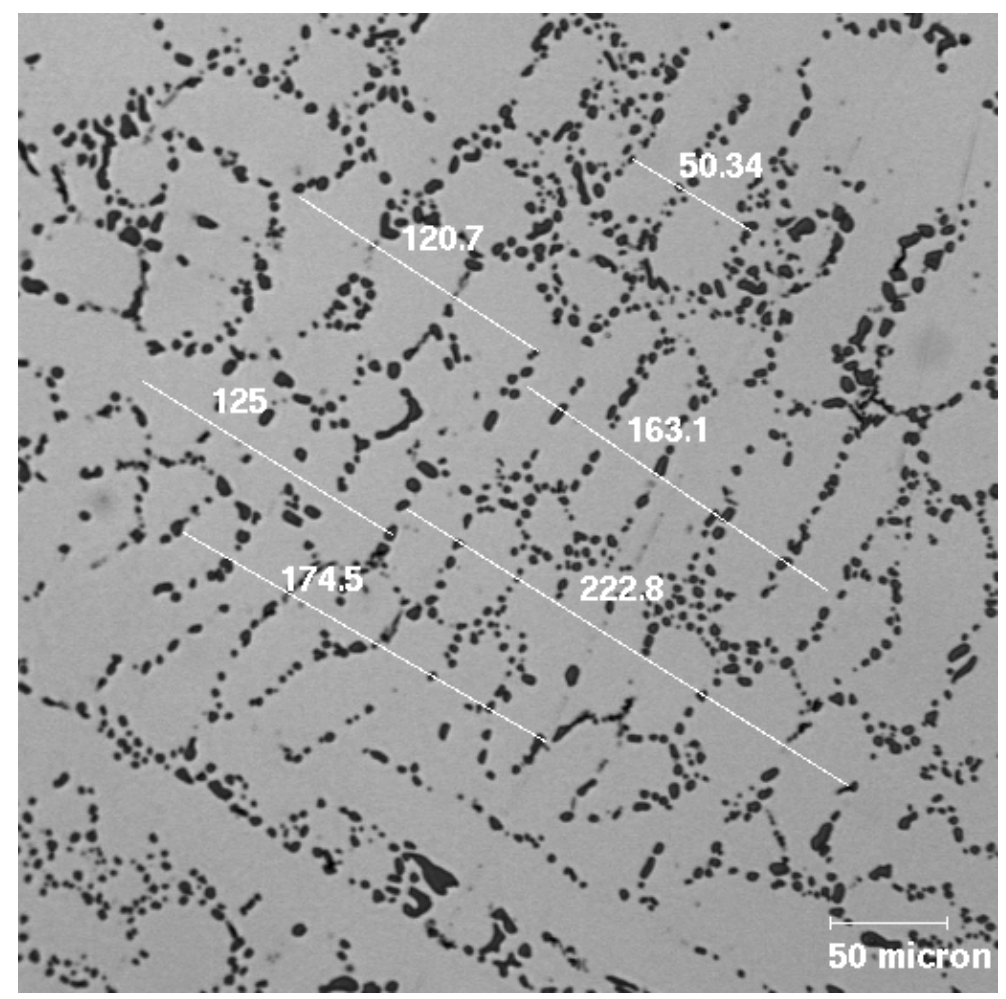

Figure 1.3-14. Measurement of SDAS of as-solutionized cast aluminum A356

\subsubsection{Effects of the solutionizing and aging time}




\section{Section 1: Thermal Prediction}

From an energy savings point of view, research has been focusing on examining the possibility of shortening the heat treatment cycle, especially reducing the solutionizing and aging time without sacrificing mechanical properties to a great extent. In this study, the first set of experiments was designed to characterize the effect of solutionizing time on the hardness of as-aged cast aluminum alloy A356 with other heat treatment parameters kept constant. The test matrix is given in Table 1.3-10. The Rockwell B hardness measurements were made on the two flats along the bar, milled down $0.381 \mathrm{~mm}$ from the surface, according to the ASTM standard. The results in Figure 1.3-15 show that the hardness drops gradually as the distance from the quench end increases. This phenomenon is due to the reduction in cooling rate along the Jominy End Quench bar, which decreases the retained supersaturation of solute available for the subsequent aging treatment. The hardness from 2 hour solutionizing is the lowest and much lower than that for the samples solutionized at the same temperature for 4, 6, 8, and 10 hours. Only a small variation in hardness is observed when solutionizing time is greater than 4 hours. This finding agrees with what was reported in the literature; so it may be concluded that 4 hours is sufficient time for solutionizing cast aluminum alloy A356 with a SDAS of $27 \mu \mathrm{m}$.

Table 1.3-10. Parameters used in the test matrix

\begin{tabular}{|c|c|c|c|c|c|}
\hline solutionizing temperature & \multicolumn{5}{|c|}{$538^{\circ} \mathrm{C}$} \\
\hline Solutionizing time (hour) & 2 & 4 & 6 & 8 & 10 \\
\hline Aging temperature & \multirow{2}{*}{\multicolumn{5}{|c|}{$\begin{array}{c}165^{\circ} \mathrm{C} \\
6\end{array}$}} \\
\hline Aging time (hour) & & & & & \\
\hline solutionizing temperature & \multirow{2}{*}{\multicolumn{5}{|c|}{$\begin{array}{c}538^{\circ} \mathrm{C} \\
6\end{array}$}} \\
\hline Solutionizing time (hour) & & & & & \\
\hline Aging temperature & \multicolumn{5}{|c|}{$165^{\circ} \mathrm{C}$} \\
\hline Aging time (hour) & 2 & 4 & 6 & 8 & 10 \\
\hline
\end{tabular}




\section{Section 1: Thermal Prediction}

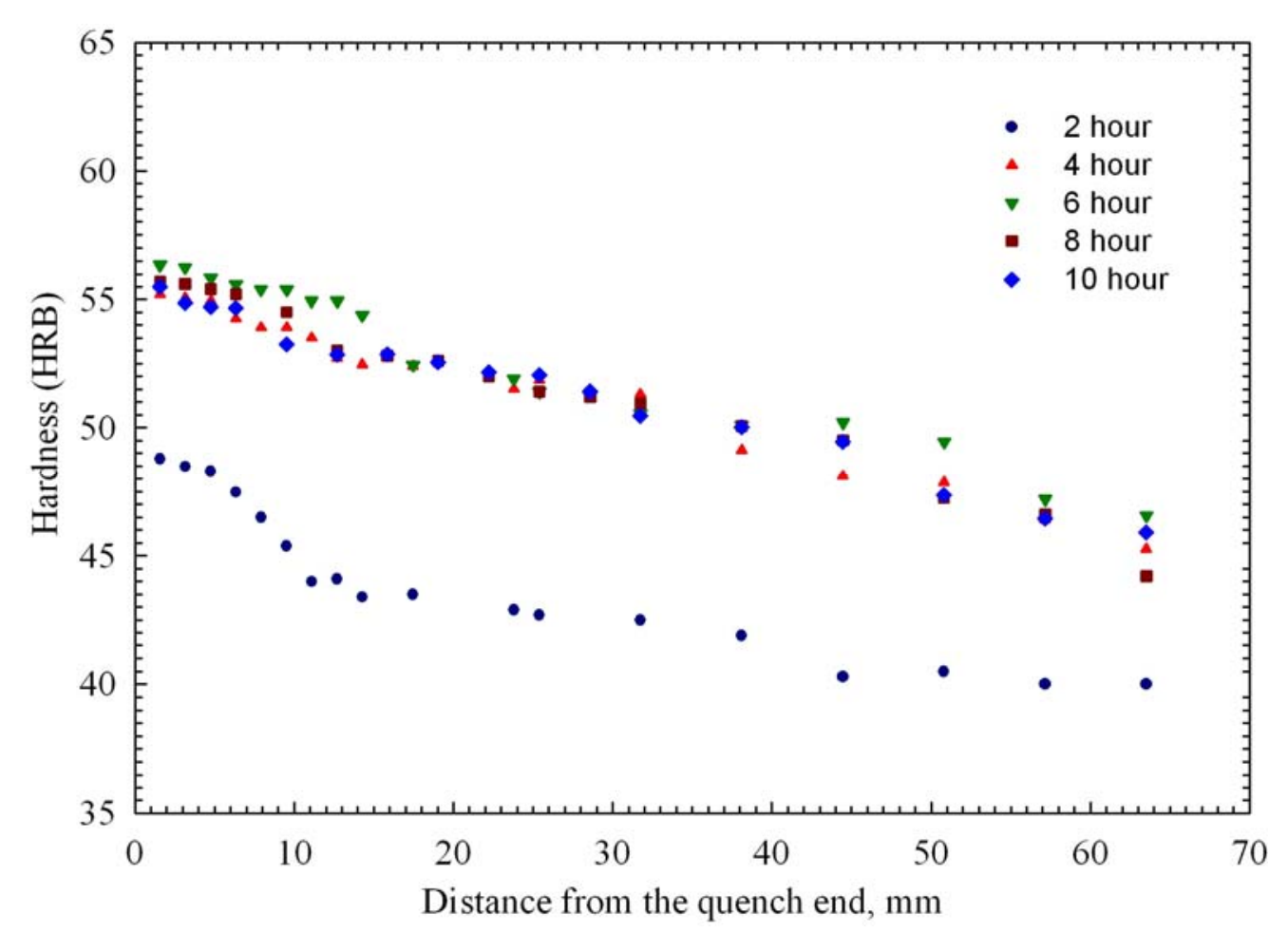

Figure 1.3-15. Hardness profile of a Jominy End Quench bar of cast aluminum alloy A356 with different solutionizing times

It is well accepted that the precipitation sequence responsible for age hardening of $\mathrm{Al}-\mathrm{Si}-\mathrm{Mg}$ alloys is based on the $\mathrm{Mg}_{2} \mathrm{Si}$ precipitates and represented by the following stages: $\alpha_{\mathrm{SSS}}(\alpha$ supersaturated solid solution) $\rightarrow$ GP zones $\rightarrow \beta^{\prime \prime} \rightarrow \beta$ ' $\rightarrow \beta$ phase $[19,20]$. The strength of the alloy is determined by the size and distribution of precipitated particles as well as the coherency of the particles with the aluminum matrix.

Based on the experimental plan given in Table 1.3-10, a series of experiments were performed to study the effect of aging time on the hardness of cast aluminum alloy A356. The results are plotted in Figure 1.3-16. A gradual decrease in hardness is observed along Jominy End Quench bars, which results from the decrease in cooling rate during the quenching process. A two-hour aging time gives the lowest hardness, which is in the range of 40HRB. Aging times greater than 2 hours increase the hardness dramatically. The highest hardness is from the 10 hour aging. 


\section{Section 1: Thermal Prediction}

In the scope of this study, the over-aging phenomenon is not seen. Aging times of 2 hours in a conventional furnace will not result in an acceptable strength of cast aluminum alloy A356.

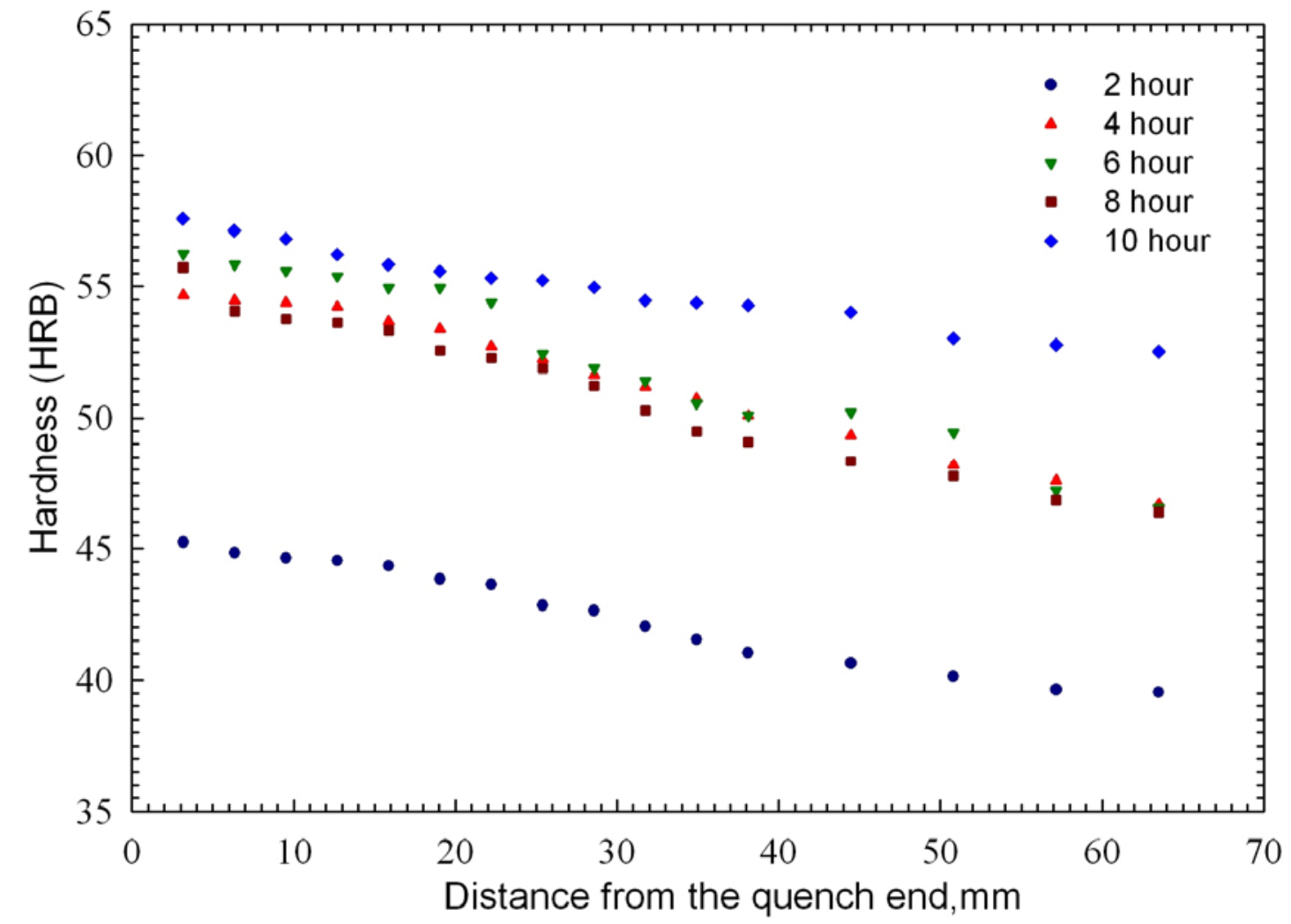

Figure 1.3-16. Hardness profile of a Jominy End Quench bar of cast aluminum alloy A356 with different aging times

Table 1.3-11. Distance from the quench end where experimental data were collected

\begin{tabular}{ccccccccccc}
\hline $\mathbf{m m}$ & 3.2 & 6.4 & 9.5 & 12.7 & 15.8 & 22.2 & 31.7 & 38.1 & 50.8 & 63.5 \\
\hline $\operatorname{Inch}(\times \mathbf{1} / \mathbf{1 6})$ & 2 & 4 & 6 & 8 & 10 & 14 & 20 & 24 & 32 & 40 \\
\hline
\end{tabular}

\subsubsection{Quench Factor modeling}

For Quench Factor modeling, both the thermal history of an alloy and the mechanical properties, which result from specific quenching rates, need to be obtained. 


\section{Section 1: Thermal Prediction}

The thermal history of cast aluminum alloy A356 was obtained by measuring the time-temperature data with K-type thermocouples during quenching process at selected locations of a Jominy End Quench bar after the bar was solutionized at $540^{\circ} \mathrm{C}$ for 4 hours. The selected locations are given in Table 1.3-11. The locations are selected to cover a wide range of cooling rates. The temperature and cooling rate profiles at different locations of a Jominy End Quench bar are presented in Figure 1.3-17 (a) and (b). Due to the nature of axial cooling along the bar, a large variation in cooling rate is observed. At the point of $3.2 \mathrm{~mm}$ from the quench end, the maximum cooling rate is approximately $150^{\circ} \mathrm{C} / \mathrm{s}$, which is equivalent to water quench. The maximum cooling rate decreases dramatically to about $5^{\circ} \mathrm{C} / \mathrm{s}$ at $63.5 \mathrm{~mm}$ from the quench end, similar to the cooling rate attainable from an air quench. A large range of cooling rates, from the fastest to the slowest, can be attained using a Jominy End Quench bar.

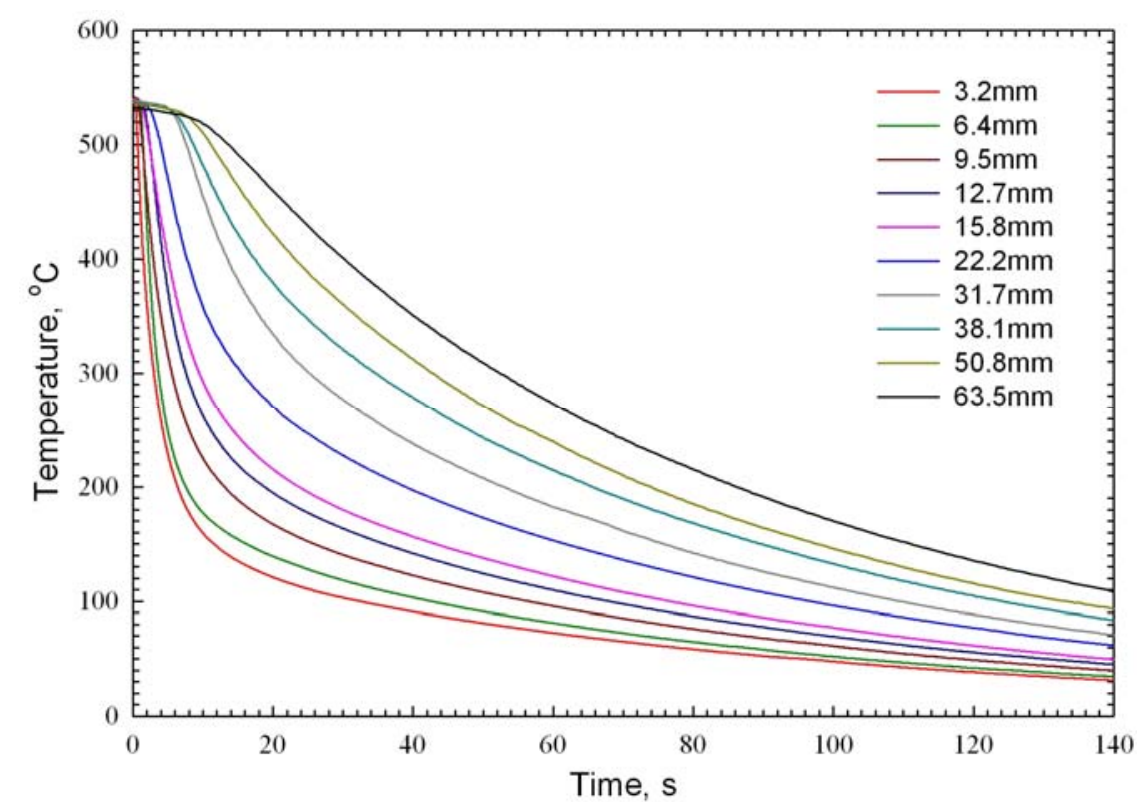

(a) Temperature vs. time 


\section{Section 1: Thermal Prediction}

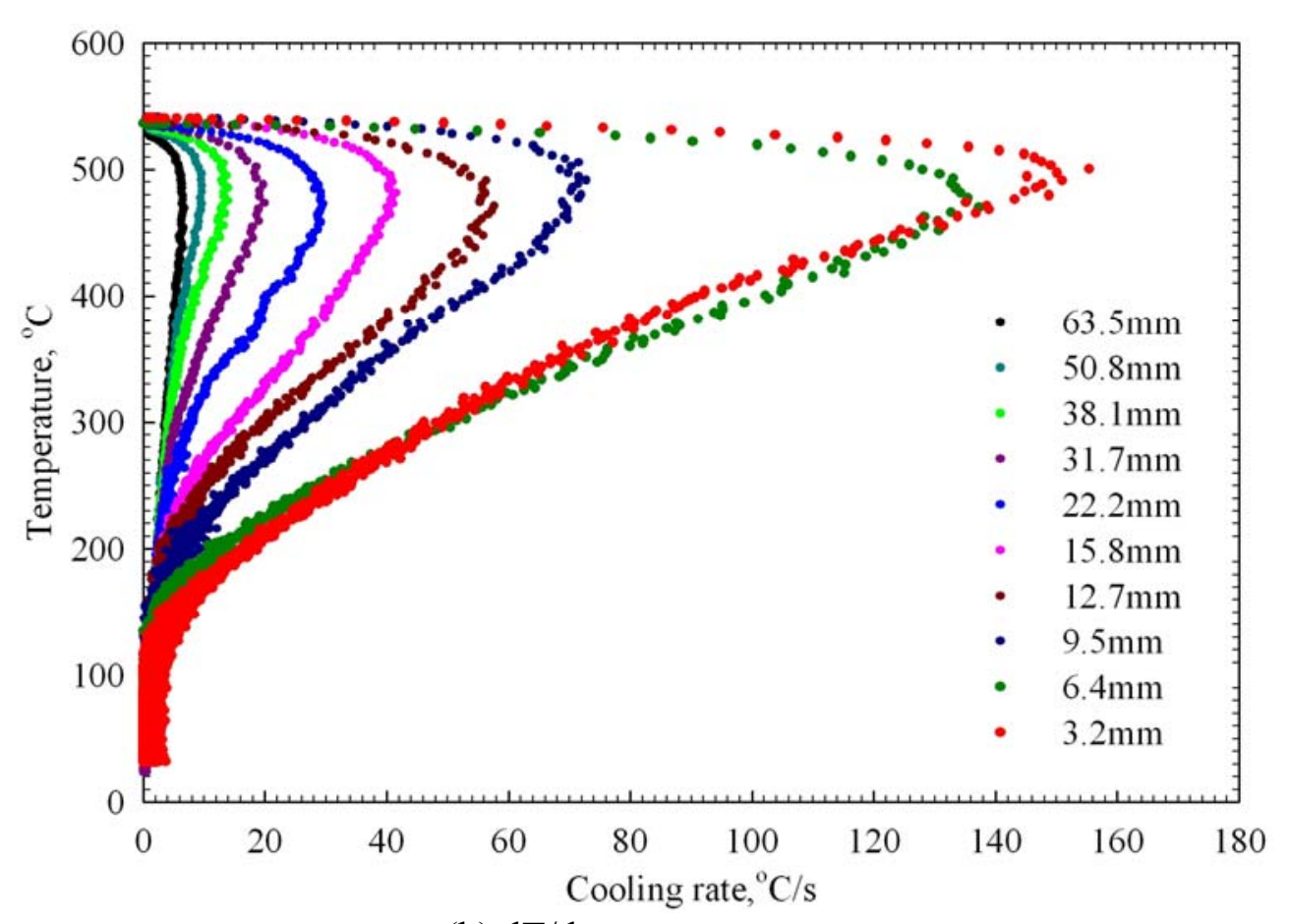

(b) dT/dt vs. temperature

Figure 1.3-17. Cooling curves (a) and cooling rate curves (b) at different locations of a Jominy End Quench bar of cast aluminum alloy A356

The mechanical property used in this analysis is the Meyer hardness, which has an approximate linear relationship with the strength; so the assumptions for the Quench Factor analysis are valid in this case. Meyer hardness values were obtained from the conversion of Rockwell B hardness values with the relationship established by Tiryakioglu and Campbell [17]. Two flats, milled down $0.381 \mathrm{~mm}$ from the surface, were machined from a Jominy End Quench bar aged for 6 hours at $165^{\circ} \mathrm{C}$. Rockwell $\mathrm{B}$ hardness measurements were made at the locations where the time-temperature data were collected. The Meyer hardness is plotted vs. distance from the quench end in Figure 1.3-18. The hardness value ranges from $143 \mathrm{MPa}$ at $3.2 \mathrm{~mm}$ from the quench end to $130 \mathrm{MPa}$ at $63.5 \mathrm{~mm}$ from the quench end. 


\section{Section 1: Thermal Prediction}

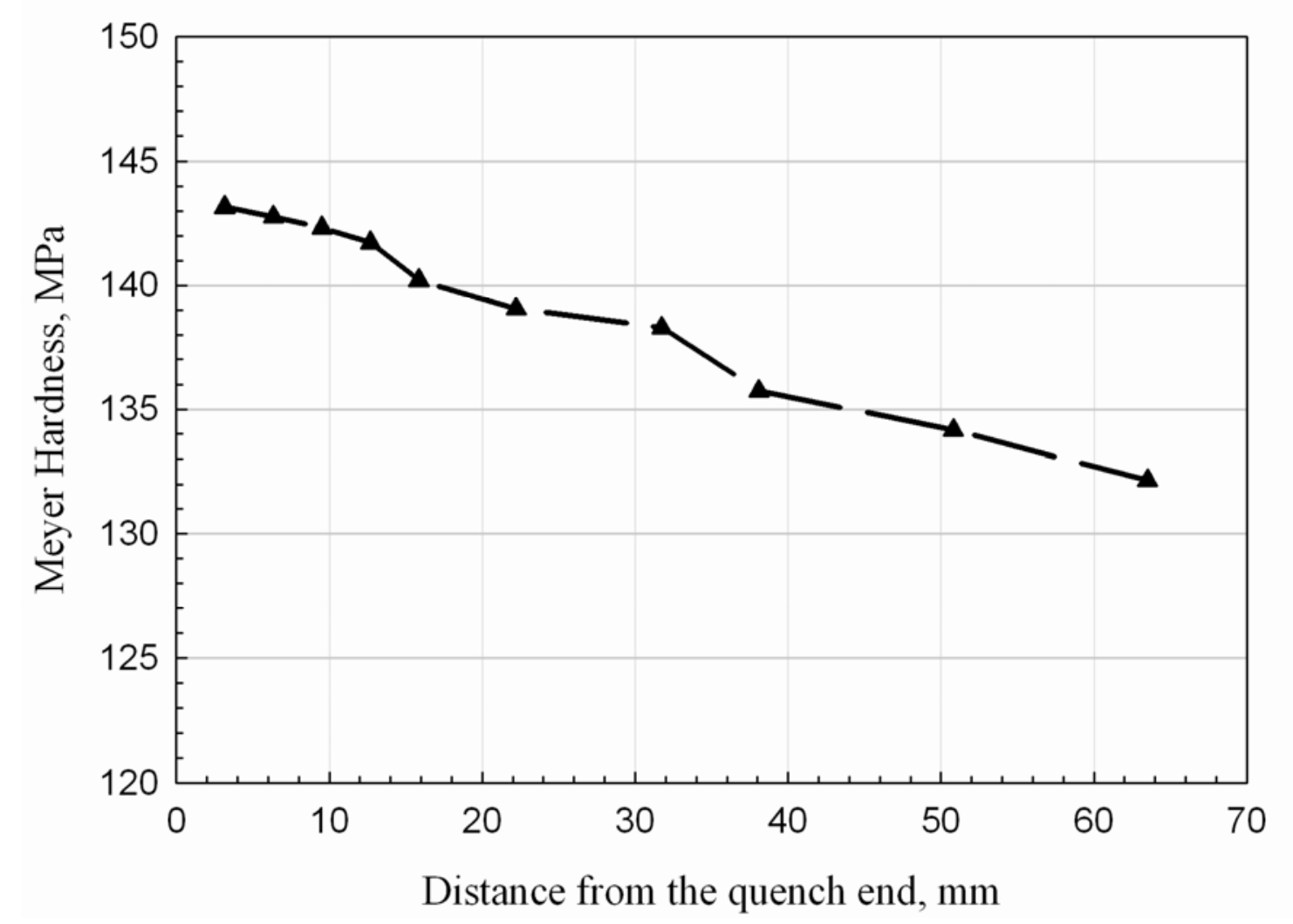

Figure 1.3-18. Meyer hardness along a Jominy End Quench bar of cast aluminum A356

The maximum Meyer hardness, $\bar{P}_{\max }$, in Equation (1.3-3) is taken as the value at the quench end since the quench end is subject to the most severe cooling and only limited precipitation is assumed to possibly occur during quenching. To obtain the minimum Meyer hardness $\bar{P}_{\min }$ in equation (1.3-3), a Jominy End Quench bar was solutionized at $540^{\circ} \mathrm{C}$ for 4 hours in a conventional furnace and then transferred to a fluidized bed that was pre-heated to $540^{\circ} \mathrm{C}$. The heater was turned off and the blower was left on. The test bar cooled slowly in the fluidized bed for about 20 hours to allow the precipitation to approach the equilibrium state [21]. The bar was then quenched in the water. The as-quenched sample was aged at $165^{\circ} \mathrm{C}$ for 6 hours in a conventional furnace. Hardness was measured on the cross section of the as-aged specimen. Ten 


\section{Section 1: Thermal Prediction}

readings were taken and averaged to obtain the minimum hardness used in the Quench Factor models.

Among the techniques available in the literature for determining the kinetic parameters, multiple linear regression analysis was employed in this paper. This technique was used by Rometsch to estimate the kinetic parameters for sand cast Al-7Si-Mg alloys in terms of yield strength [21]. Instead of minimizing the squares of the difference between the predicted and measured property as described in the least squares routine, this method is used to obtain a best linear relationship between a function of experimentally measured properties and the calculated Quench Factors.

If double natural logarithms are taken on both sides of Equation (1.3-3), then the following equation is generated. Since the relationship between strength and Quench Factor in Equation (1.3-3) is valid, the logarithm of fractional Meyer hardness has a linear relationship with $\operatorname{Ln}(\mathrm{Q})$ with the intercept being Avrami exponent n, as shown in Equation (1.3-7).

$$
\operatorname{Ln}\left[\frac{1}{K_{1}} \operatorname{Ln}\left(\frac{\sigma-\sigma_{\min }}{\sigma_{\max }-\sigma_{\min }}\right)\right]=n \operatorname{Ln}(Q)
$$

The left side of the equation can be calculated with the known maximum, minimum hardness, and the measured hardness at the selected locations of a Jominy End Quench bar. Together with experimentally measured quenching data in Figure 1.3-17, K constants in Equation (1.3-1) are initially estimated to calculate the Quench Factors Q at the same locations using Equation (1.3-2). The logarithm of fractional Meyer hardness is plotted against $\operatorname{Ln}(\mathrm{Q})$ as a scatter plot. The scatter plot is fitted with a linear curve and coefficient of determination $\left(\mathrm{R}^{2}\right)$ for the curve is calculated [21]. The constants in Equation (1.3-1) are iteratively adjusted until the hypothetical Quench Factors provide the highest possible coefficient of determination for the plot while the fitted linear curve passes through the origin (or the intercept is very close to 0) [21].

An example best-fit curve using Equation (1.3-3) is shown in Figure 1.3-19. The kinetic parameters and Avrami exponent obtained from multiple linear regression analysis are presented 


\section{Section 1: Thermal Prediction}

in Table 1.3-12. The constants for the improved model in Equation (1.3-4) are obtained by the same analysis and presented in Table 1.3-12.

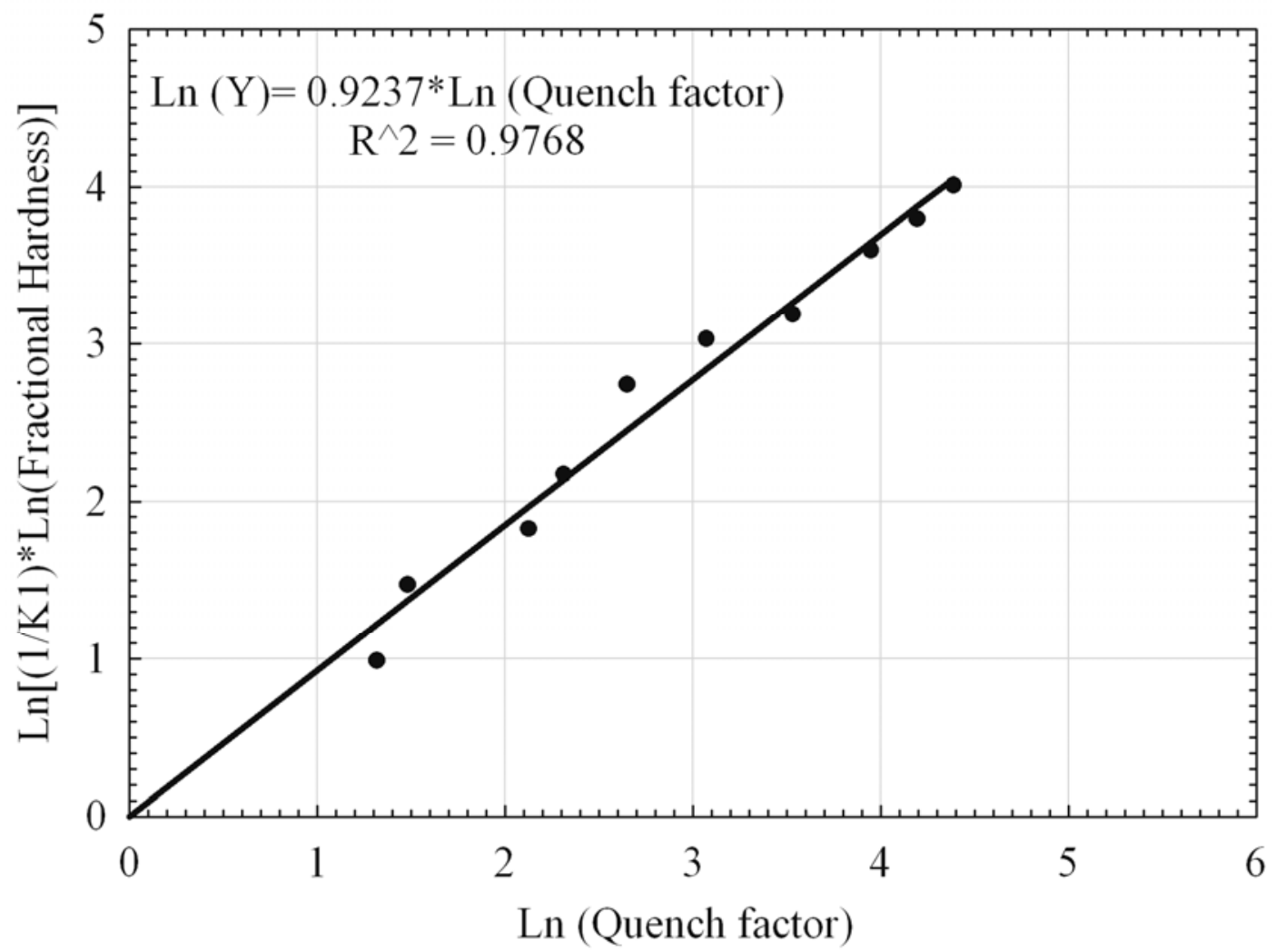

Figure 1.3-19. An example best fit curve for Quench Factor analysis of cast aluminum alloy A356 (0.5\% precipitation)

Table 1.3-12. Precipitation kinetic parameters of cast aluminum alloy A356 during quenching

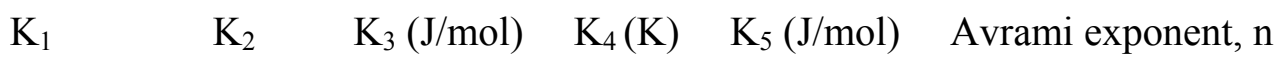

\section{Equation}

$$
\text { (1.3-3) }
$$

$$
-0.005013 \quad 1.27 \mathrm{E}-09
$$

60

$764 \quad 131000$

0.92

\section{Equation}

$\begin{array}{lllllll}\mathbf{( 1 . 3 - 4 )} & -0.005013 & 6.41 \mathrm{E}-10 & 56 & 764 & 131000 & 0.92\end{array}$




\section{Section 1: Thermal Prediction}

With the constants given in Table 1.3-12, the critical times were calculated using Equation (1.3-1) and plotted as a function of temperature for both original and improved Quench Factor models, as shown in Figure 1.3-20. These two curves correspond to $0.5 \%$ precipitation for cast aluminum alloy A356.

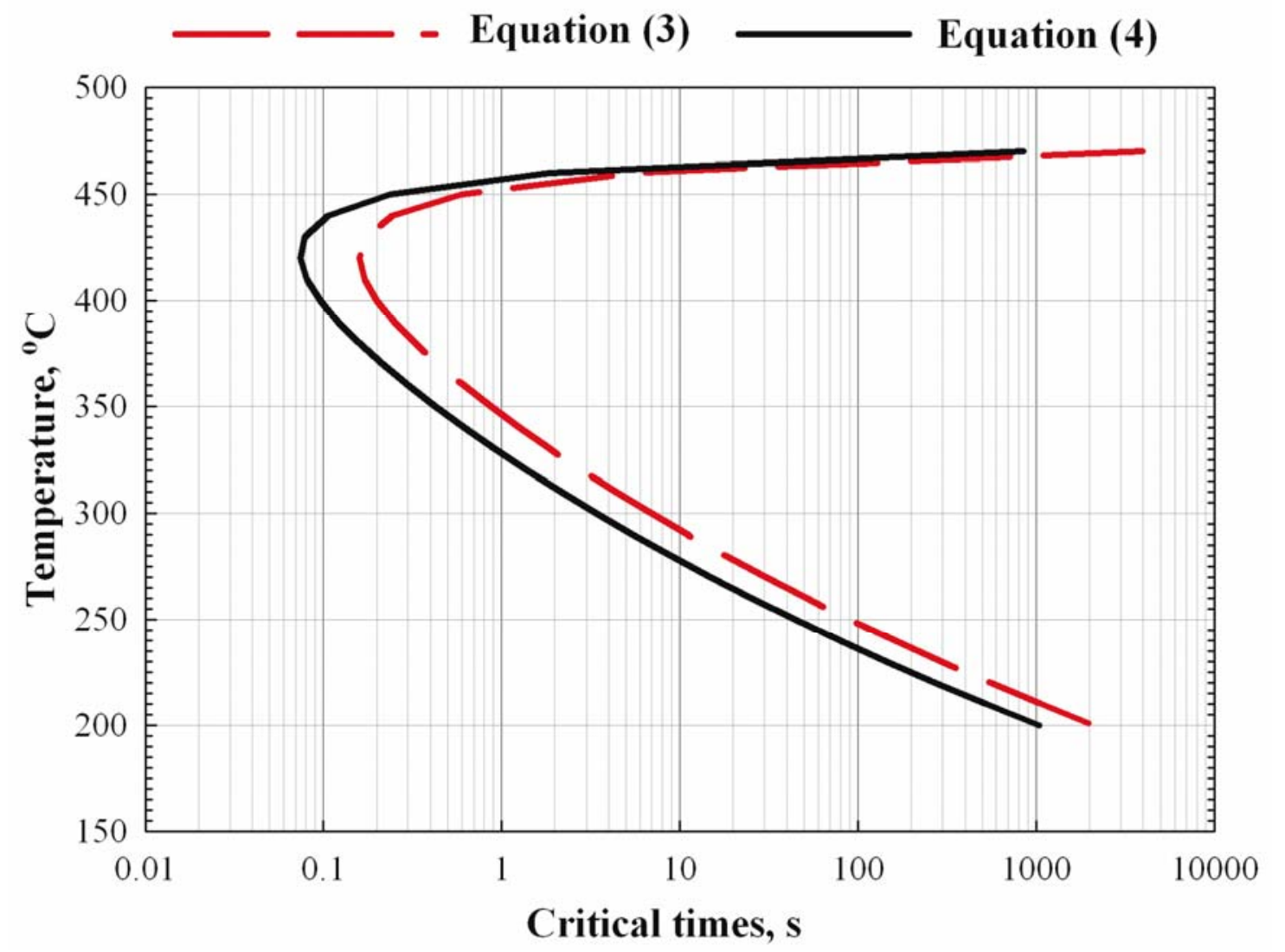

Figure 1.3-20. Time-Temperature-Property (TTP) curves for cast aluminum alloy A356

\subsubsection{Experimental verification}

Experimental verification was performed using five-cylinder cast aluminum A356 engine cylinder head, which was cast using the lost foam casting process. Sixty four engine heads were placed in a quench load, in 2 layers $(2 \times 32)$ in a continuous furnace, as shown in Figure 1.3-21 [22]. One of the engine heads was instrumented with K-type thermocouples to record the 


\section{Section 1: Thermal Prediction}

time-temperature data during the quenching process. One engine head was selected for the purpose of mechanical testing and metallographic investigation. The rest of the engine cylinder heads were used as dummies to study the effect of racking pattern.
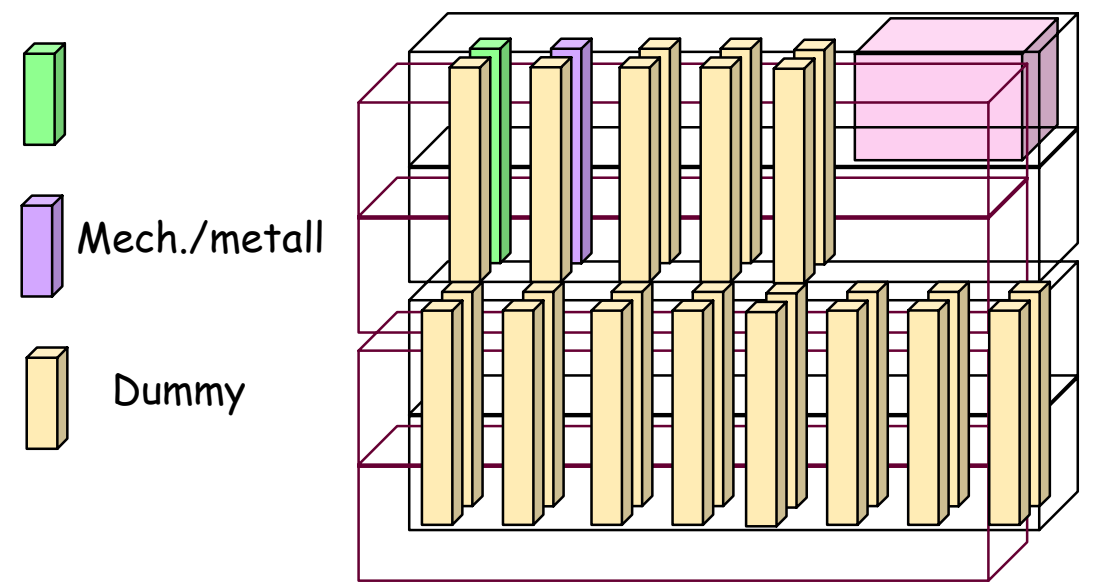

Figure 1.3-21. Racking pattern of cast aluminum A356 engine heads in a continuous furnace

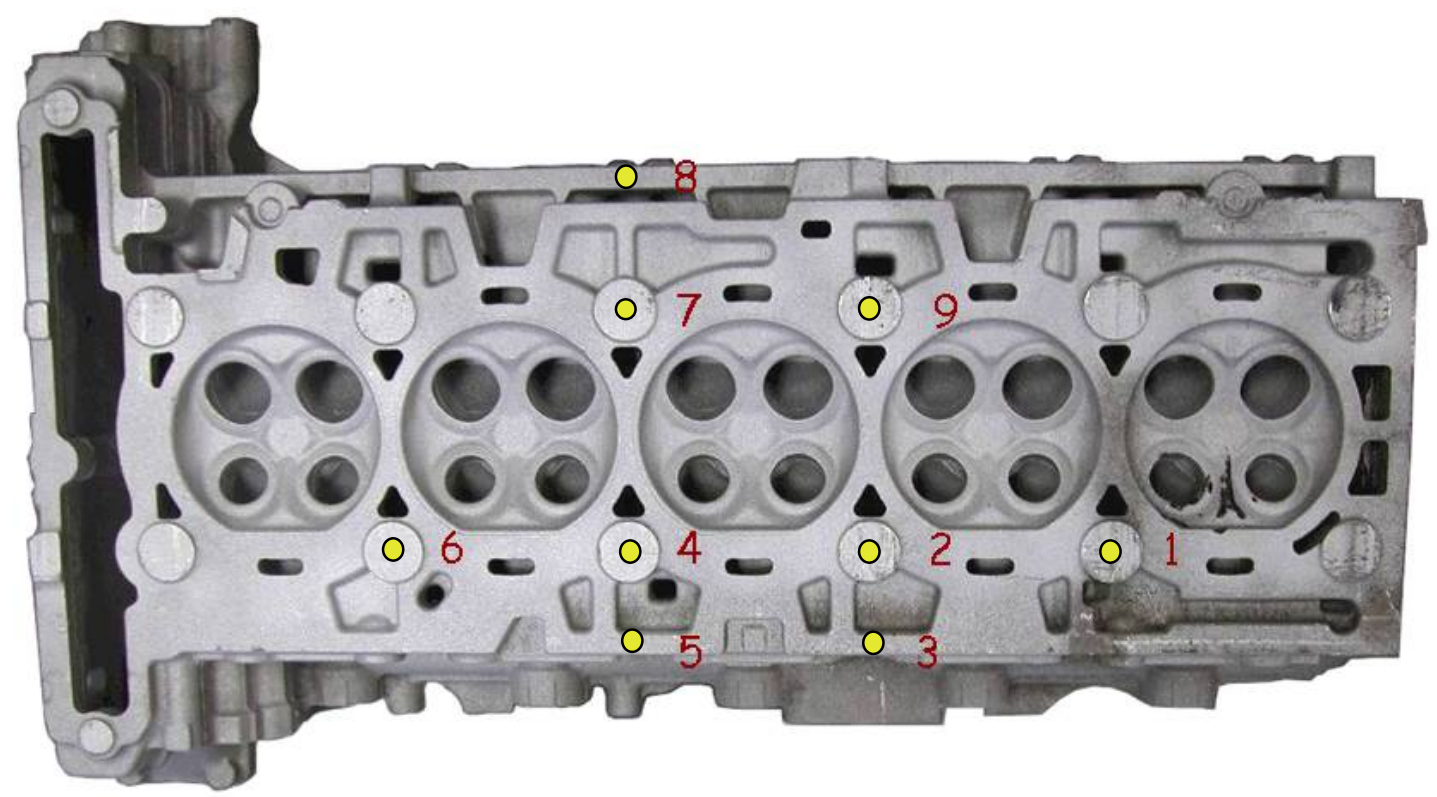

Figure 1.3-22. Cast aluminum A356 engine head instrumented with K-type thermocouples [22] 


\section{Section 1: Thermal Prediction}

The engine heads were solutionized at $538^{\circ} \mathrm{C}\left(1000^{\circ} \mathrm{F}\right)$ for 5 hours in a continuous furnace $(7$ hours including the ramp-up time) and quenched in agitated water at $76^{\circ} \mathrm{C}\left(170^{\circ} \mathrm{F}\right)$. As shown in Figure 1.3-22, K-type thermocouples were instrumented at the selected 9 locations of one five-cylinder engine head and time-temperature data were collected at these locations during quenching process. As-quenched engine cylinder heads were aged at $160^{\circ} \mathrm{C}\left(320^{\circ} \mathrm{F}\right)$ for 4 hours (6 hours including the ramp-up time). As-aged samples were used for metallography and mechanical testing.

Table 1.3-13. Predicted and measured hardness of a cast A356 engine head

$\begin{array}{lcc} & \text { Location 7 } & \text { Location 8 } \\ \text { Measured hardness (HRB) } & 58.5( \pm 0.8) & 59.4( \pm 1.0) \\ \text { Predicted hardness (Equation (1.3-3)) } & 59.0 & 59.5 \\ \text { Predicted hardness (Equation (1.3-4)) } & 58.9 & 59.3\end{array}$

Two specimens were removed from the locations where thermocouples 7 and 8 were attached to the cast aluminum A356 engine head. Rockwell B hardness measurements were taken near the spot where the thermocouple tips were attached using a Wilson hardness tester Model 3JR, S/N 10661. The results are shown in Table 1.3-13. Using the time-temperature data collected at the corresponding two locations, the Meyer hardness was predicted with the kinetic parameters given in Table 1.3-12 and converted to Rockwell B hardness. The predicted hardness data were compared with the measured hardness, with the results shown in Table 1.3-13. The predicted hardness agreed well with the experimental result. These results have also been presented elsewhere [22]. 


\section{Section 2: Solutionizing Prediction}

The software developed for the numerical simulation of solidification, post-solidification cooling, and solution heat treatment is composed of four major modules. The flow during execution of the software is illustrated in Figure 2.0-1. The CASTSEG module simulates the evolution of microstructure and solute distribution during solidification and during cooling of the casting in the mold after solidification. The CASTSOLN module simulates the evolution of microstructure and solute distribution during post-solidification thermal cycles, in particular, solution heat treatment. To execute the simulations both CASTSEG and CASTSOLN require continual access to phase equilibrium and diffusivity data. The PHASECALC module provides access to stored phase equilibria data to CASTSEG and CASTSOLN. The DIFFCALC module provides access to stored diffusivity data to CASTSEG and CASTSOLN. The major functions of the PHASECALC module are presented in section 2.1. The major functions of the DIFFCALC module are presented in section 2.2. The major functions of the CASTSEG module are presented in section 2.3. The major functions of the CASTSOLN module are presented in section 2.4 .

Alloy and casting parameters needed as input to the solidification simulation routines, CASTSEG, can be entered by the user, can be taken from a stored data file, or can be input from a comprehensive solidification (heat transfer) package. Process and thermal parameters needed as input to the solution heat treatment routines, CASTSOLN, can be entered by the user, accessed from a data file, or transferred by the thermal prediction software described in section 1.0 of this DOE report.

The numerical simulation routines have been designed to be flexible and efficient in the prediction of the microstructure and solute distribution during processing of aluminum casting alloys with the major alloying elements silicon, copper, and/or magnesium. The models that underlie the simulation routines and the stored thermodynamic and kinetic data have been tailored to the phase transformation and mass transfer behavior of multicomponent and multiphase aluminum alloys typically specified for automotive applications. In principle the routines can be applied to any precipitation/age hardenable alloy system. To obtain accurate 


\section{Section 2: Solutionizing Prediction}

predictions, the models validated for aluminum alloys would need to be compared for the additional alloy systems and detailed, reliable thermodynamic and kinetic data would have to be obtained.

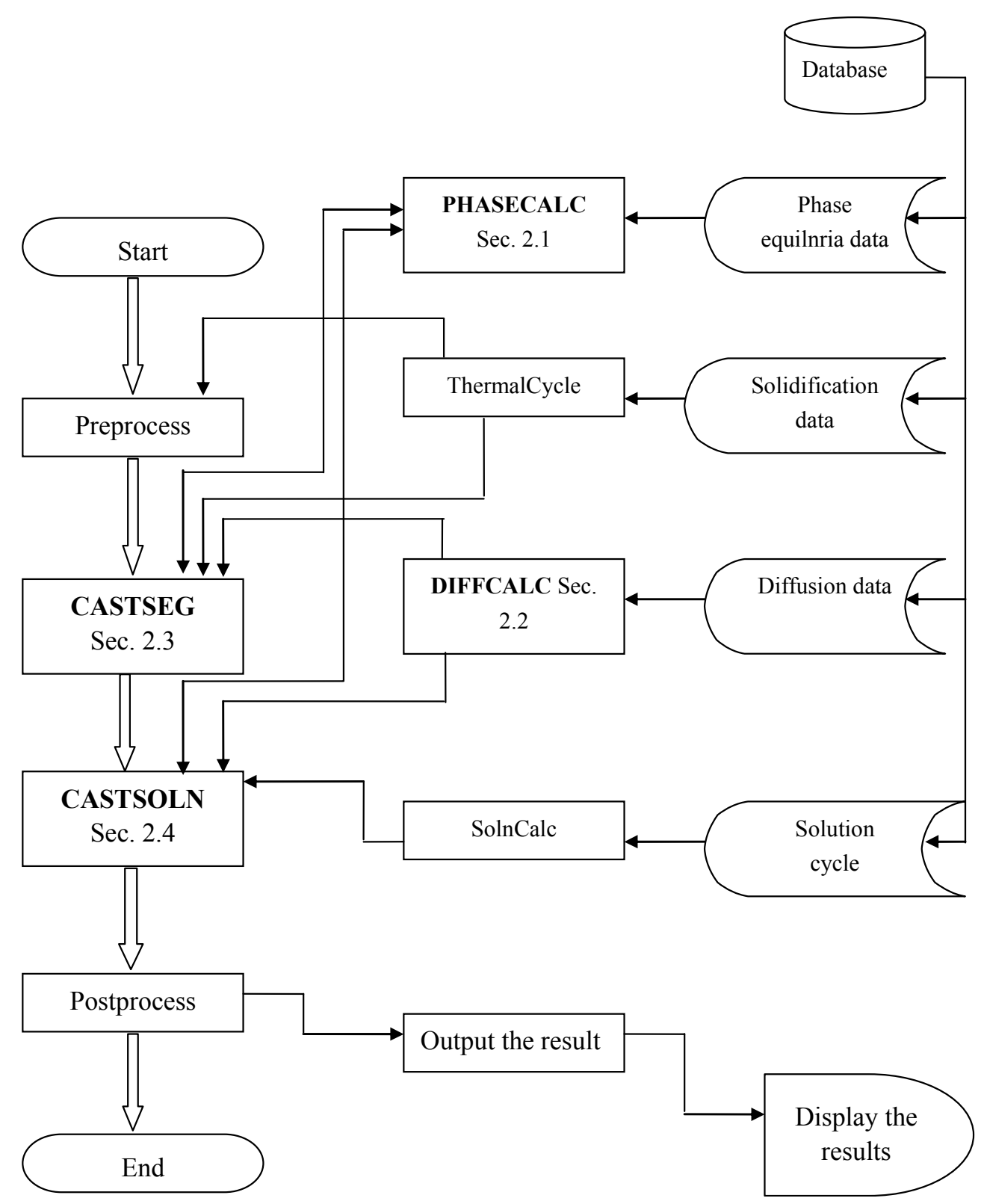

Figure 2.0-1. Overall flow diagram for routines to simulate evolution of microstructure and solute distribution during solidification, post-solidification cooling, reheating, and solution heat treatment for aluminum-base casting alloys with silicon, copper, and/or magnesium 


\section{Section 2: Solutionizing Prediction}

\subsection{PHASECALC}

The major functions of PHASECALC are to represent and access phase equilibria data for multicomponent alloys in the temperature and composition ranges relevant to solidification and solution heat treatment for the relevant casting alloys. The required data include: (a.) the stable phases within a given range of compositions and temperatures and (b.) the maximum solubility of each alloying element in each stable phase as a function of temperature. The data input to PHASECALC are obtained from the database tasks reported in section 3.4. The phase relations are determined from published experimental data, thermodynamic calculation, and from our experiments. PHASECALC is required to represent the phase equilibria at least to the precision and the accuracy of the input data.

The representation of phase equilibria by the computer software should facilitate the computations that depend on phase relations. One commonly required procedure in solidification and heat treatment simulation is to find the point on a liquidus or solvus boundary appropriate to a known composition; for example, given the composition of the liquid, find its liquidus temperature. Another common procedure is to find the equilibrium composition of one or more phases when the composition (and temperature) of another phase in a multiphase region is specified.

This DOE project has focused on Al-Si-Cu-Mg (Al-319) and Al-Si-Mg (Al-356) casting alloys. In addition to the primary alloying elements, silicon, magnesium, and copper, iron is a significant component of commercial aluminum casting alloys. Typically, manganese and zinc also are present in commercial aluminum alloys. Titanium and boron are added for grain refinement. Strontium and sodium are added for modification of the morphology of the silicon constituent. For aluminum casting alloys eight to ten components have significant influence on determining the final microstructure and properties. In developing databases and implementing software we have focused on the phase equilibria for aluminum casting alloys containing

$$
5<\% \mathrm{Si}<9,0<\% \mathrm{Mg}<0.5,0<\% \mathrm{Cu}<5,0<\% \mathrm{Fe}<0.6 \% \text {. }
$$




\section{Section 2: Solutionizing Prediction}

The phase equilibria representation and access procedures are based on the shape (interpolation) functions used in finite element analyses. The data representation and data access procedures are described below, in terms of shape functions, provide the basis for representation of binary, ternary, quaternary and higher order multi-component alloy systems.

\section{$\underline{\text { 2.1.1 Form Factors for Binary Systems }}$}

Consider a simple binary eutectic phase diagram between solute B and solvent A, in Figure 2.1-1. The phase diagram indicates the regions of stability for phases in temperature $(T)$ and composition (C) space. Unless otherwise stated the units of composition are weight percent (100 x kg element/kg alloy).

Subscripts are unnecessary to represent compositions in a binary alloy. The solute and solvent concentrations are not independent; they add to $100 \%$; and the solute concentration, alone, can be used to represent the composition of the alloy.

A superscript is used to represent a phase: the superscript $L$ to represent the liquid phase, the superscript $\alpha$ to represent the primary solid solution phase, and the superscript $\beta$ to represent the secondary phase, which may be an intermetallic compound or solid solution. When no superscript is used, the overall composition of the alloy is indicated. Then initial overall solute content of the alloy would be $C_{O}$, and the initial composition of a melt, prior to solidification, would be represented by $C_{O}^{L}$.

In the region marked $\mathrm{L}$ in Figure 2.1-1, the liquid solution is the only stable phase. The liquidus curve is the lower boundary for the single-phase liquid region. The liquidus indicates the locus of temperatures and compositions where the melt becomes saturated with respect to solvent or solute. For a eutectic system, the liquidus boundary has a minimum point at the eutectic liquid composition and eutectic temperature $\left(C_{E}^{L}, T_{E}\right)$. 


\section{Section 2: Solutionizing Prediction}

The solidus curve is the lower boundary for stability of the liquid phase. In Figure 2.1-1 the solidus curve includes the $\tilde{\alpha-s o l i d u s, ~ t h e ~ e u t e c t i c ~ i s o t h e r m, ~ a n d ~ t h e ~} \tilde{\beta}$-solidus. The liquidus and the solidus boundaries touch at the eutectic isotherm in Figure 2.1-1 and, in general, at points of temperature invariant solidification.

In the region marked $\alpha$ in Figure 2.1-1, the $\alpha$-solid solution is the only stable phase. The $\tilde{\alpha-s o l i d u s ~ a n d ~} \tilde{\alpha-s o l v u s}$ form the boundary for the single phase, primary solid solution region. The $\alpha$-solidus and $\alpha$-solvus mark the limits of the single-phase $\alpha$-region and the locus of compositions and temperatures of saturation of $\alpha$-solid solution with respect to solvent. The $\tilde{\alpha-s o l i d u s ~ b o u n d a r y ~ i n d i c a t e s ~ t h e ~ l o c u s ~ o f ~ c o m p o s i t i o n s ~ a n d ~ t e m p e r a t u r e s ~ a b o v e ~ t h e ~ e u t e c t i c ~}$ temperature where the primary solid phase, $\alpha$, becomes saturated with respect to solute. The $\alpha$-solvus boundary indicates the locus of compositions and temperatures below the eutectic temperature where the primary solid $\alpha$-phase becomes saturated with respect to solute.

Single-phase regions are separated by regions where two phases are stable. The stable phases in the two-phase regions are the stable phases in the neighboring single-phase regions. Above the eutectic temperature, the temperature range relevant to simulation of solidification processes, the two phase regions are liquid plus alpha-phase and liquid plus beta phase. Below the eutectic temperature, the temperature range relevant to the simulation of thermal treatment processes, the two-phase region is a mixture of the solid phases alpha plus beta.

Only at the eutectic temperature are three phases simultaneously stable. The $\alpha$-phase, $\tilde{\beta-p h a s e}$, and liquid phases can coexist, at equilibrium, at the eutectic temperature. The eutectic isotherm touches the three single-phase regions, at their solubility limits, and abuts the two-phase regions.

Relevant to PHASECALC and the next three modules to be discussed, is the relation of degrees of freedom and the geometric dimensionality of phase stability regions on a phase diagram. Single-phase regions in binary alloys are two-dimensional, 2-D, represented by an area, and the alloy has two degrees of freedom within the single-phase region. The temperature and 


\section{Section 2: Solutionizing Prediction}

composition of the alloy may change independently within the region, and the alloy will remain single phase.

On the other hand, for temperatures and compositions that fall in the regions labeled $\mathrm{L}+\alpha_{2} . \mathrm{L}+\beta$, and $\alpha+\beta$, the temperature and composition of the phases cannot change independently. For the alloy to remain in equilibrium in the two phase $\alpha+\beta$ region, for example, the temperature and composition of the $\alpha$-phase must change along the one dimensional, $1-\mathrm{D}, \alpha$-solvus and the temperature and composition of the $\tilde{\beta}$-phase must change along the $\beta$-solvus line. The alloy has only a single degree of freedom. If a two phase $\alpha+\beta$ alloy is held at a single temperature, a solution treatment temperature $\left(T_{S}\right)$ for example, the condition for stable equilibrium is that the composition of the $\alpha$-phase must be at the point of intersection of the isotherm and the $\alpha$-solvus $\left(C_{S}^{\alpha}, T_{S}\right)$ and the $\tilde{\beta}$-phase must be at the point of intersection of the isotherm and the $\tilde{\beta \text {-solvus }}$ $\left(C_{S}^{\beta}, T_{S}\right)$. The isotherm with end points defined by $C_{S}^{\alpha}$ and $C_{S}^{\beta}$ is called a tie line. The storage and accessing of equilibrium tie lines are the central tasks for the PHASECALC module.

The compositions of the phases in a two-phase region are fixed but the weight fractions of the phases $\left(f^{\alpha}, f^{\beta}\right)$ vary to satisfy the overall mass balance, known as the lever rule:

$$
f^{\alpha}=\frac{\left(C^{\beta}-C_{O}\right)}{\left(C^{\beta}-C^{\alpha}\right)} \text { and } f^{\beta}=\frac{\left(C_{O}-C^{\alpha}\right)}{\left(C^{\beta}-C^{\alpha}\right)}
$$

The lever rule is analogous to the interpolation functions to be introduced shortly. For any composition that falls along the tie line

$$
f^{\alpha}+f^{\beta}=1
$$

At the end point on the $\tilde{\alpha}$ solvus $\mathrm{C}_{\mathrm{O}}=\mathrm{C}^{\mathrm{a}}, f^{\alpha}=1$ and $f^{\beta}=0$, etc. 


\section{Section 2: Solutionizing Prediction}

For a binary alloy, three-phase equilibrium has zero degrees of freedom. The compositions of the three phases and the temperature are fixed to the three zero-dimensional (0-D) points: $\left(C_{E}^{L}, T_{E}\right),\left(C_{E}^{\alpha}, T_{E}\right)$, and $\left(C_{E}^{\beta}, T_{E}\right)$.

In essence, PHASECALC uses a series of several straight-line segments to represent a curved phase boundary for a binary alloy. The number and length of the segments are selected to best represent the curvature of the phase boundary. The data are stored as arrays in data files named for the specific alloy system. Each of the phase boundaries in a binary alloy system is a curve that can be expressed as $T^{\phi}=f\left(C^{\phi}\right)$ where $C^{\phi}$ is the concentration of solute on the $\phi$-phase boundary at temperature $T^{\phi}$. Figure 2.1-2 shows the liquidus and solidus boundaries on the A-rich end of the phase diagram as curved solid lines. Often representation of curved phase boundaries as single straight lines in solidification simulation routines will result in predictions of as-cast microstructures with insufficient accuracy for the compositions and phase fractions to be used for input to heat treatment simulations.

The bold black lines in Figure 2.1-2 represent the line segments selected to represent the liquidus and solidus boundaries. The lengths of the segments, typically, are not equal. The number and temperatures of the endpoints of the line segments on the liquidus and solidus boundaries should be the same. The respective end points, then, will represent a tie line. For a binary alloy system the main data stored and accessed are the array of points $\left(\mathrm{T}_{\mathrm{i}}, \mathrm{C}_{\mathrm{i}}{ }^{\alpha}, \mathrm{C}_{\mathrm{i}}^{\mathrm{L}}\right)$ and $\left(\mathrm{T}_{\mathrm{i}}, \mathrm{C}_{\mathrm{i}}^{\alpha}, \mathrm{C}_{\mathrm{i}}^{\beta}\right)$ that represent the tie lines connecting the $\alpha$-solidus and $\alpha$-liquidus and the $\alpha$-solvus and $\beta$-solvus, respectively.

Consider the endpoints of a line segment on the liquidus boundary to be $\left(C_{i}^{L}, T_{i}^{L}\right)$ and $\left(C_{j}^{L}, T_{j}^{L}\right)$. The linear equation for the line segment can be written as

$$
T^{L}=m^{L} C^{L}+b^{L}
$$




\section{Section 2: Solutionizing Prediction}

where $m^{L}$ and $b^{L}$ are constants that can be found by evaluating the equation (2.1-3) at the end points, $\mathrm{i}$ and $\mathrm{j}$.

$$
\begin{aligned}
& {\left[\begin{array}{c}
T_{i} \\
T_{j}
\end{array}\right]=\left[\begin{array}{ll}
C_{i}^{L} & 1 \\
C_{j}^{L} & 1
\end{array}\right]\left[\begin{array}{c}
m^{L} \\
b^{L}
\end{array}\right]} \\
& {\left[\begin{array}{c}
m^{L} \\
b^{L}
\end{array}\right]=\left[\begin{array}{ll}
1 & 0 \\
0 & 1
\end{array}\right]\left[\begin{array}{c}
\frac{T_{j}-T_{i}}{C_{j}^{L}-C_{i}^{L}} \\
T_{j}-\frac{T_{i}}{C_{j}^{L}-C_{i}^{L}} C_{j}^{L}
\end{array}\right]}
\end{aligned}
$$

Once the constants $m^{L}$ and $b^{L}$ have been evaluated, the liquidus temperature, $\mathrm{T}^{\mathrm{L}}$, can be found for the specified liquid composition, $\mathrm{C}^{\mathrm{L}}$.

Alternatively, equation (2.1-3) can be rearranged to provide the liquidus temperature by linear interpolation.

$$
T^{L}=N_{i}^{L} T_{i}+N_{J}^{L} T_{j}
$$

where the shape functions, $N_{i}^{L}$ and $N_{j}^{L}$, are the ratios in the parentheses below.

$$
N_{i}^{l}=\left(\frac{C_{j}^{L}-C^{L}}{C_{j}^{L}-C_{1}^{L}}\right) \quad \text { and } N_{j}^{L}=\left(\frac{C^{L}-C_{i}^{L}}{C_{j}^{L}-C_{i}^{L}}\right)_{j}
$$

Analogous to the lever rule, equation (2.1-2), the shape functions sum to unity.

$$
N_{i}^{L}+N_{j}^{L}=1
$$

and at the end points the shape function of the same subscript equals 1 and the other shape function equals 0 . At a composition half way between the end points both shape functions equal $1 \frac{1}{2}$. For compositions that do not fall between the endpoints on the line segment one shape function will be greater than 1 and the other less than 0 . 


\section{Section 2: Solutionizing Prediction}

The representation of phase equilibria by storing a series of equilibrium tie lines and using shape functions to interpolate between stored data points facilitate the solute redistribution computations in the solidification (CASTSEG) and heat treatment (CASTSOLN) simulations. As stated above a commonly required procedure is to find the point on a liquidus or solvus boundary appropriate to a known composition. A second common procedure is to find the equilibrium composition of one or more phases when the composition (and temperature) of another phase in a multiphase region is given. Stated in terms of a binary alloy system, the first procedure would be to find the point $\left(\mathrm{T}^{\mathrm{L}}, \mathrm{C}^{\mathrm{L}}\right)$ on the liquidus boundary given the composition of the liquid is $\mathrm{C}^{\mathrm{L}}$. The second procedure would be to find the point $\left(\mathrm{T}^{\mathrm{L}}, \mathrm{C}^{\alpha}\right)$ on the solidus boundary that determines the composition of the alpha phase that can be in equilibrium with liquid of composition $\mathrm{C}^{\mathrm{L}}$ at temperature $\mathrm{T}^{\mathrm{L}}$, where $\mathrm{C}^{\alpha}$ and $\mathrm{C}^{\mathrm{L}}$ are the compositions at the end points of the tie line at $\mathrm{T}^{\mathrm{L}}$ connecting the $\alpha$-solidus and $\alpha$-liquidus.

The first part of the procedure to locate a liquidus temperature for a given liquid composition, $\mathrm{C}^{\mathrm{L}}$, would be to successively evaluate shape functions $(2.1-5 b)$ for the line segments until a line segment is found where both shape functions are between (or equal to) 0 and 1 . Then the shape functions are substituted in equation 2.1-5a to find the liquidus temperature, $\mathrm{T}^{\mathrm{L}}$.

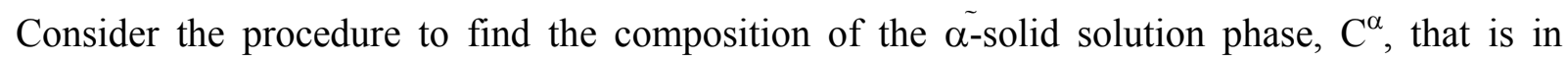
equilibrium with the liquid of composition $\mathrm{C}^{\mathrm{L}}$ at temperature, $\mathrm{T}^{\mathrm{L}}$. Noting that the end points of the line segments representing solidus and liquidus are taken at the same temperatures, the shape factors for the $\alpha$-phase can be equated to the shape factors for the liquid, $N_{i}^{L}=N_{i}^{\alpha}$ and $N_{j}^{L}=N_{j}^{\alpha}$. The composition of the a-phase can be computed using a relation analogous to equation (2.1-5a).

$$
C^{\alpha}=N_{i}^{\alpha} C_{i}^{\alpha}+N_{J}^{\alpha} C_{j}^{\alpha}
$$

\subsubsection{Form Factors for Ternary Systems}




\section{Section 2: Solutionizing Prediction}

The representation of higher order, multi-component phase diagrams will be discussed using a ternary eutectic alloy system as an example. The phase diagram, as for binary systems, represents the regions of phase stability in temperature composition space. For a binary system there is only one independent composition. For an $\mathrm{n}$ component alloy, there are $\mathrm{n}-1$ independent composition variables. For a ternary alloy system the coordinates of the phase diagram would be $\mathrm{C}_{1}, \mathrm{C}_{2}$, $\mathrm{T}$, where the subscripts denote the two alloying elements. The ternary phase diagram (at constant pressure) is three-dimensional $\left(\mathrm{T}, \mathrm{C}_{1}, \mathrm{C}_{2}\right)$.

Single-phase regions have three degrees of freedom and are represented by a volume. For example, the terminal solid solution $\alpha$-phase region is a three-dimensional (3-D) volume in which the temperature and two solute compositions can be changed independently.

Two-phase regions have two degrees of freedom and are bounded by two 2-D surfaces. For example, the liquidus and solidus boundaries for the $\mathrm{L}+\alpha$ region are curved surfaces. Isothermal tie lines connect each point on the liquidus surface $\left(\mathrm{T}, \mathrm{C}_{1}{ }^{\mathrm{L}}, \mathrm{C}_{2}{ }^{\mathrm{L}}\right)$ to a unique point on the solidus surface $\left(\mathrm{T}, \mathrm{C}_{1}{ }^{\alpha}, \mathrm{C}_{2}{ }^{\mathrm{L}}\right)$ to which it can be in equilibrium.

Three phase equilibria have one degree of freedom and the 1-D curves that relate the allowed compositions and temperature are called lines of two-fold saturation. The compositions that can be in equilibrium are connected by an isothermal tie triangle.

Four-phase equilibrium, in a ternary system, can occur at a single temperature. For example, for a ternary system, the eutectic equilibrium occurs at four points on an isothermal plane. A unique liquid composition is in equilibrium with three solid phases whose compositions are fixed at unique points connected by an isothermal tie triangle that encloses the composition of the 1liquid. (Note that the maximum number of phases that can exist at equilibrium is $n+1$.)

Figure 2.1-3 illustrates an isothermal cut through a ternary phase diagram. Note the axes to represent the compositions of solute 1 and solute 2 form a sixty-degree angle. Known as a Gibbs triangle these axes facilitate the representation of three component systems. The apex to 


\section{Section 2: Solutionizing Prediction}

the left represents pure base metal, A. The adjacent legs of the Gibbs triangle would represent the binary phase diagrams for A - solute 1 alloys and A - solute 2 alloys.

In the cut through the ternary phase diagram in Figure 2.1-3, the isotherm shown is above the melting point of component 2 and below the melting point of component 1 and the base metal. The phase stability regions shown are three single-phase regions $\mathrm{L}, \alpha$, and $\beta$, and two, two phase regions $L+\alpha$ and $L+\beta$. The curved lines are the intersection of the isothermal plane with the liquidus and solidus surfaces. The series of tie lines shown in the two-phase regions connect the solid and liquid compositions that would be in equilibrium at this temperature. When tie line information is not complete, it rarely is, the tie lines are assumed to be uniformly distributed along the liquidus and solidus boundaries.

In Figure 2.1-4 the intersections of several isothermal planes with the liquidus surface are projected on a single Gibbs triangle. Also shown are the projections of several flat triangular plane segments that are used to represent the curved liquidus surface. Again the number of triangular planes and their end points are selected to adequately represent the curvature of the phase boundary. The linear equation for a flat plane is

$$
T=m_{1}^{L} C_{1}^{L}+m_{2}^{L} C_{2}^{L}+b^{L}
$$

where $m_{1}^{L}, m_{2}^{L}$, and $b^{L}$ are constants that describe each plane. For a triangle with endpoints at $\mathrm{i}, \mathrm{j}$, and $\mathrm{k}$, the constants can be determined by evaluating the relation 2.1-7 at the three endpoints.

$$
\left[\begin{array}{c}
T_{i} \\
T_{j} \\
T_{k}
\end{array}\right]=\left[\begin{array}{ccc}
C_{i 1}^{L} & C_{i 2}^{L} & 1 \\
C_{j 1}^{L} & C_{j 2}^{L} & 1 \\
C_{k 1}^{L} & C_{k 2}^{L} & 1
\end{array}\right]\left[\begin{array}{c}
m_{1}^{L} \\
m_{2}^{L} \\
b^{L}
\end{array}\right]
$$

Once $m_{1}^{L}, m_{2}^{L}$, and $b^{L}$ have been determined and substituted into equation $2.1-7$, the equation can be rearranged in terms of the shape functions.

$$
T=N_{i}^{L} T_{i}+N_{j}^{L} T_{j}+N_{k}^{L} T_{k}
$$




\section{Section 2: Solutionizing Prediction}

Again,

$$
N_{i}^{L}+N_{j}^{L}+N_{k}^{L}=1
$$

At i, $N_{i}^{L}=1, N_{j}^{L}=0$, and $N_{k}^{L}=0$, etc.

Given a composition for the liquid, the liquidus temperature can be found by a procedure parallel to that used for binary alloys. The shape factors for successive triangles can be evaluated until a triangle, $\mathrm{i}, \mathrm{j}, \mathrm{k}$, is found in which all shape factors are between (or equal to) 0 and 1 . Then the temperature is found from equation 2.1-10. Also, similarly to the procedure for a binary alloy, given a liquid composition and temperature, the composition of the solid in equilibrium with the liquid can be determined. The corresponding triangle on the solidus surface is located, the shape functions for the solidus are set equal to the shape functions for the liquidus, and the definition of the shape function is used to find the composition.

\subsubsection{Form Factors for Quaternary and Higher Order Systems}

For an n-component system, there will be $\mathrm{n}-1$ independent composition variables, $\mathrm{C}_{\mathrm{i}}$. Using the aluminum rich end of the quaternary system $\mathrm{Al}-\mathrm{Si}-\mathrm{Cu}-\mathrm{Mg}$ as illustration, the three independent composition variables will be $\mathrm{C}_{\mathrm{Si}}, \mathrm{C}_{\mathrm{Cu}}$, and $\mathrm{C}_{\mathrm{Mg}}$. The coordinates of the quaternary phase diagram (at constant pressure) are $\left(\mathrm{T}, \mathrm{C}_{\mathrm{Si}}, \mathrm{C}_{\mathrm{Cu}}, \mathrm{C}_{\mathrm{Mg}}\right)$. The quaternary phase diagram will be four-dimensional (4-D) and, in general, the phase diagram for an n-component system will be n-dimensional. Representation of a 4-D and higher dimensional phase diagrams on two-dimensional sheets of paper becomes increasingly complex.

Single-phase regions will be n-dimensional and the bounding surfaces; i.e., the liquidus, solidus, and solvus boundaries, would be n-1 dimensional. For the Al-Si-Cu-Mg system the liquid and $\alpha$-phase regions would be four-dimensional; temperature and three composition variables could be changed independently within the single-phase regions. 


\section{Section 2: Solutionizing Prediction}

The liquidus, solidus, and solvus surfaces would be three-dimensional. Three-dimensional, 3-D, surfaces would form the boundaries for each two-phase region. For example, the liquid and $\alpha$-phases in equilibrium at a specified temperature would be connected by a tie line connecting points on the liquidus and solidus surfaces. The intersection of an isothermal plane with the 3-D liquidus surface would be a 2-D surface.

The three-phase regions; e.g. $\mathrm{L}+\alpha+$ silicon, would be bounded by three 2-D surfaces of two-fold saturation. The equilibrium compositions of the three phases would be connected by a tie triangle.

The four phase regions would bounded by four curved lines. The equilibrium compositions would be found as the points of intersection of the isothermal plane for the specified temperature with the four curved lines.

For the quaternary Al-Si-Cu-Mg system, the maximum number of phases that could be in equilibrium would be five. The quaternary eutectic for $\mathrm{Al}-7 \% \mathrm{Si}-3.5 \% \mathrm{Cu}-0.5 \% \mathrm{Mg}$ alloys would be $\mathrm{L} \rightarrow \alpha$-phase + silicon-phase + Q-phase $+\theta$-phase $\left(\mathrm{CuAl}_{2}\right)$.

The scheme for the storage of phase equilibrium data for a quaternary system, in general, is illustrated in Figures 2.1-5 and 2.1-6 and described more specifically for the Al-Si-Cu-Mg system in the following. Representation of the two phase region in the quaternary system would be storage of the temperature and end points of a large number of equilibrium tie lines; e.g. for the $\mathrm{L}+$ $\alpha$ region, each equilibrium tie line would be represented by

$$
T_{i} \quad C_{i-S i}^{L} \quad C_{i-C u}^{L} \quad C_{i-M g}^{L} \quad C_{i-S i}^{\alpha} \quad C_{i-C u i}^{\alpha} \quad C_{i-M g}^{\alpha}
$$

Representation of the three-phase region would be by the temperature and the three equilibrium compositions on the end points of the tie triangle:

$$
T_{j} \quad C_{j-S i}^{L} \quad C_{j-C u}^{L} \quad C_{j-M g}^{L} \quad C_{j-S i}^{\alpha} \quad C_{j-C u}^{\alpha} \quad C_{j-M g}^{\alpha} \quad C_{j-S i}^{\text {silicon }} \quad C_{j-C u}^{\text {silicon }} \quad C_{j-M g}^{\text {silicon }}
$$




\section{Section 2: Solutionizing Prediction}

As stated above, $C_{j-S i}^{\text {silicon }}=100 \%, \quad C_{j-C u i}^{\text {silicon }}=0, C_{j-M g}^{\text {silicon }}=0$.

The liquidus surface may be considered to be represented by a series of terahedra. The tetrahedra are formed by four neighboring points. The tetrahedral approximate the 3-D liquidus surface of the quaternary system analogously to the approximate representation of the 2-D liquidus surface of a ternary system by a series of triangles. (Note figure 2.1-4.) To find the temperature on the liquidus surface associated with a specified liquid composition, the data points stored for the two phase $\mathrm{L}+\alpha$-region are searched to find the four points closest to the specified composition and for which all four shape factors are between 0 and 1 . Then the associated temperature is determined by linear interpolation.

$$
T=N_{i}^{L} T_{i}^{L}+N_{j}^{L} T_{j}^{L}+N_{k}^{L} T_{k}^{L}+N_{l}^{L} T_{l}^{L}
$$

The composition of the $\alpha$-phase in equilibrium with the specified liquid would be found by applying the same four shape factors to the corresponding tetrahedron on the solidus surface.

The three 2-D surfaces that bound three-phase regions in the quaternary system would be represented by a series of flat triangular planes using procedures analogous to those applied to the liquidus, solidus, and solvus surfaces that bound two-phase regions in ternary systems. The four curved lines that bound four-phase regions in the quaternary system would be represented by a series of straight-line segments using procedures analogous to the lines of two-fold saturation that bound the three phase regions in ternary systems and the liquidus, solidus, and solvus boundaries for two-phase regions in binary systems.

The procedures described can be used to represent alloy systems with any number of components. It is difficult for us to visualize boundaries and regions that have more than three dimensions. But the phase rule, that governs the number of degrees of freedom (degrees of freedom $=$ number of components - number of phases +1 ), is a thermodynamic requirement that will govern the 


\section{Section 2: Solutionizing Prediction}

phase equilibria. The task of obtaining accurate input data becomes increasingly difficult as the number of components increases.

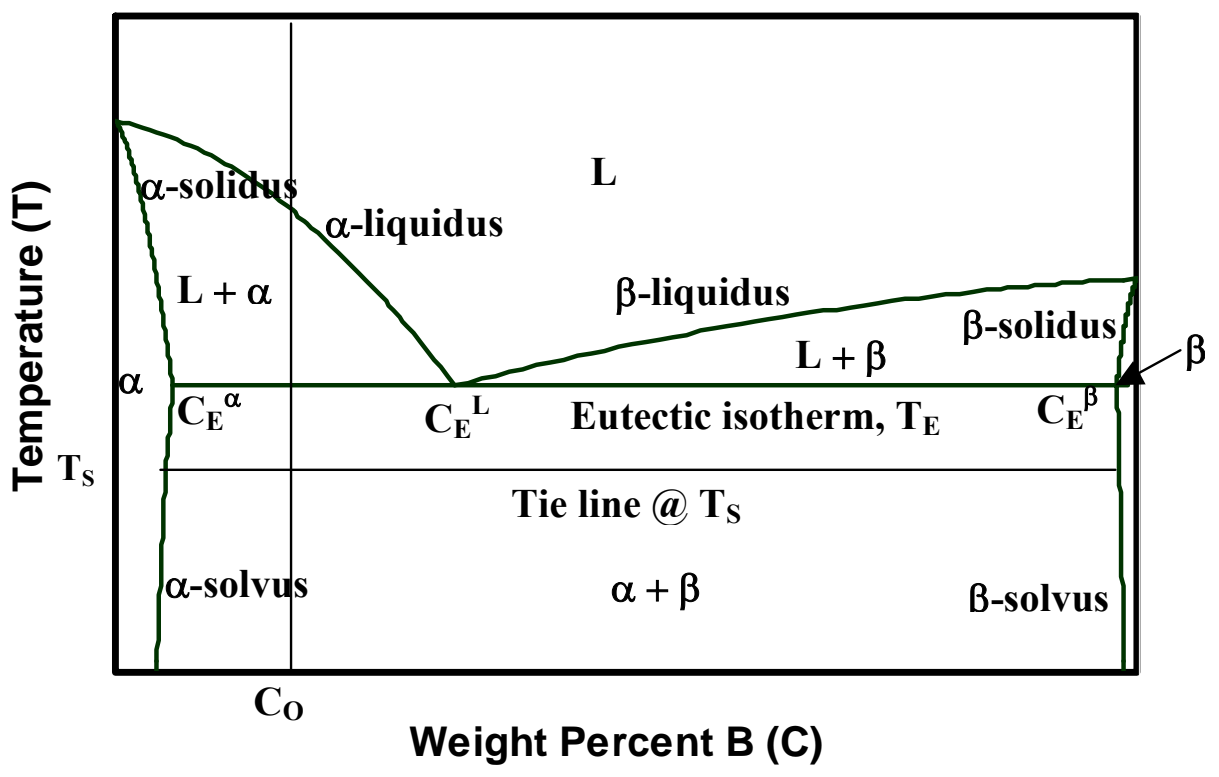

Figure 2.1-1. Binary phase diagram between elements A and B to illustrate phase equilibria data represented by module PHASECALC

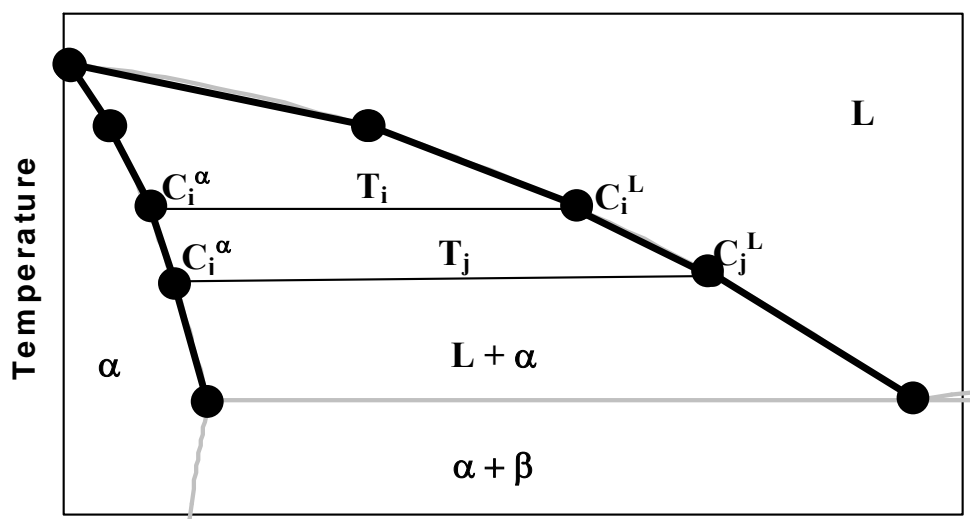

Weight Percent $B(C)$

Figure 2.1-2. Representation of curved liquidus and solidus boundaries by four straight-line segments, each. The phase boundaries are solid light gray curves and the simulated phase boundaries are the bold black line segments 


\section{Section 2: Solutionizing Prediction}

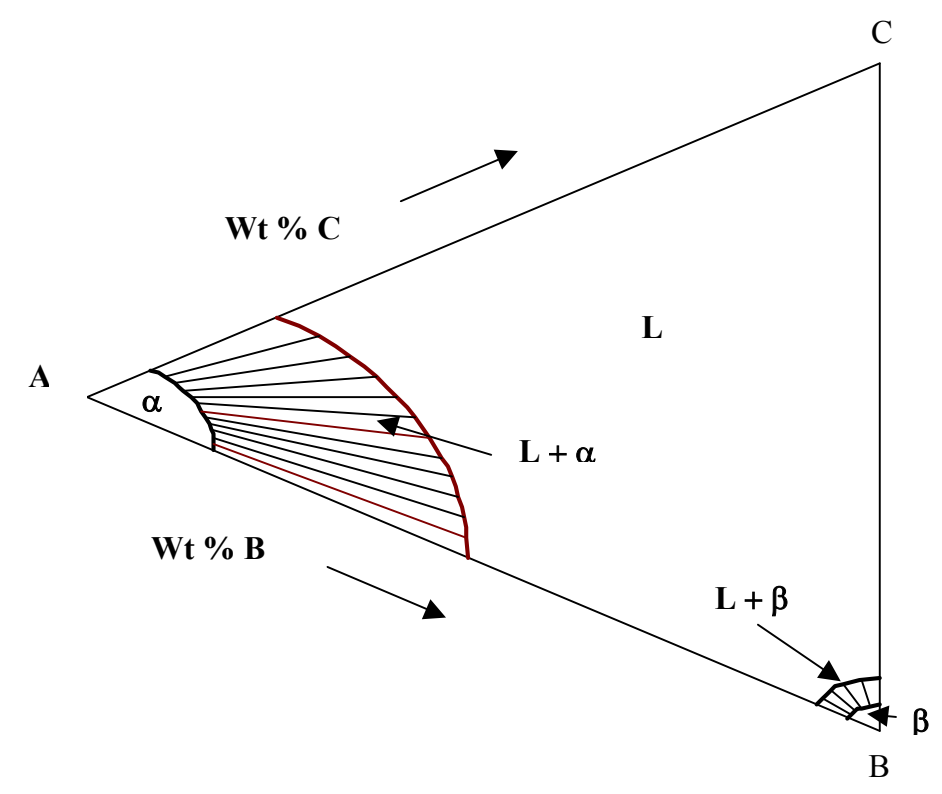

Figure 2.1-3. Isothermal section through the A-B-C ternary alloy system at a temperature above the melting point of $\mathrm{C}$ and below the melting points of $\mathrm{A}$ and $\mathrm{B}$

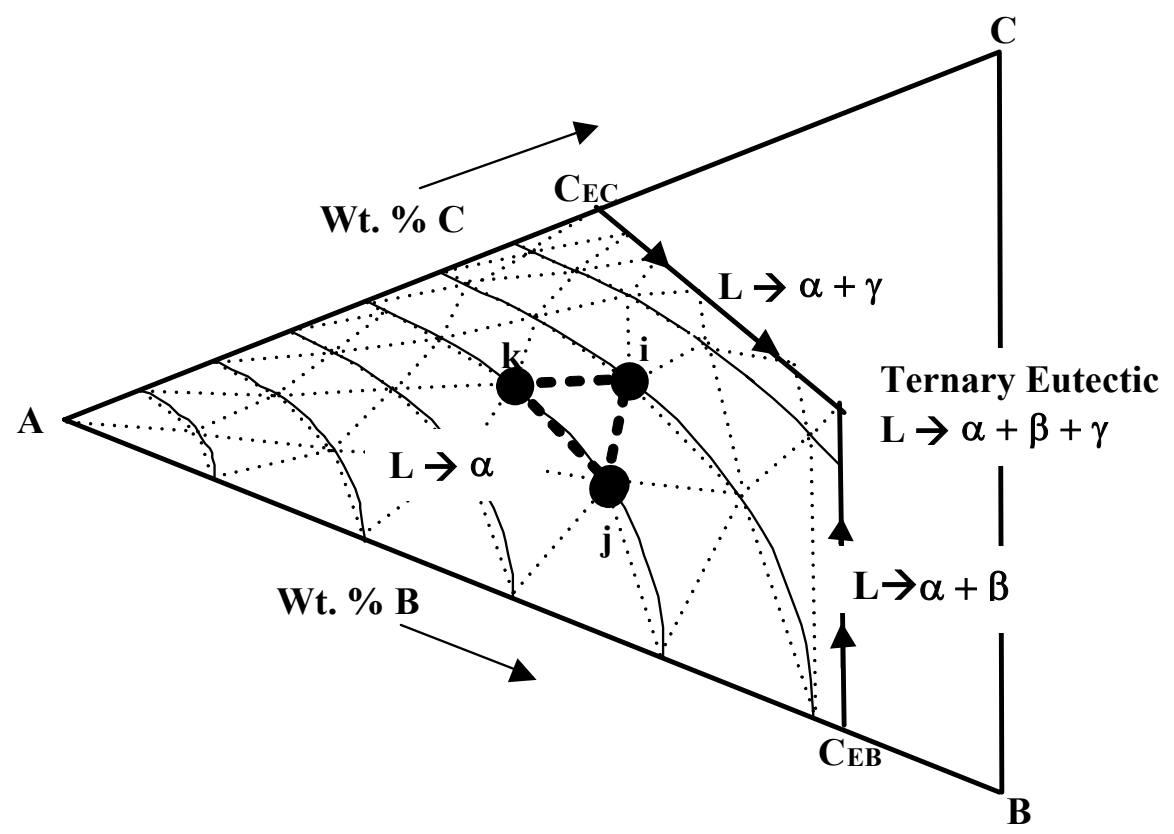

Figure 2.1-4. Projection of several liquidus isotherms (solid lines) on a basal plane. Dotted triangles are flat triangular planes to represent the curved liquidus surface. The three corners of each triangular plane are labeled $\mathrm{i}, \mathrm{j}$, and $\mathrm{k}$ 


\section{Section 2: Solutionizing Prediction}

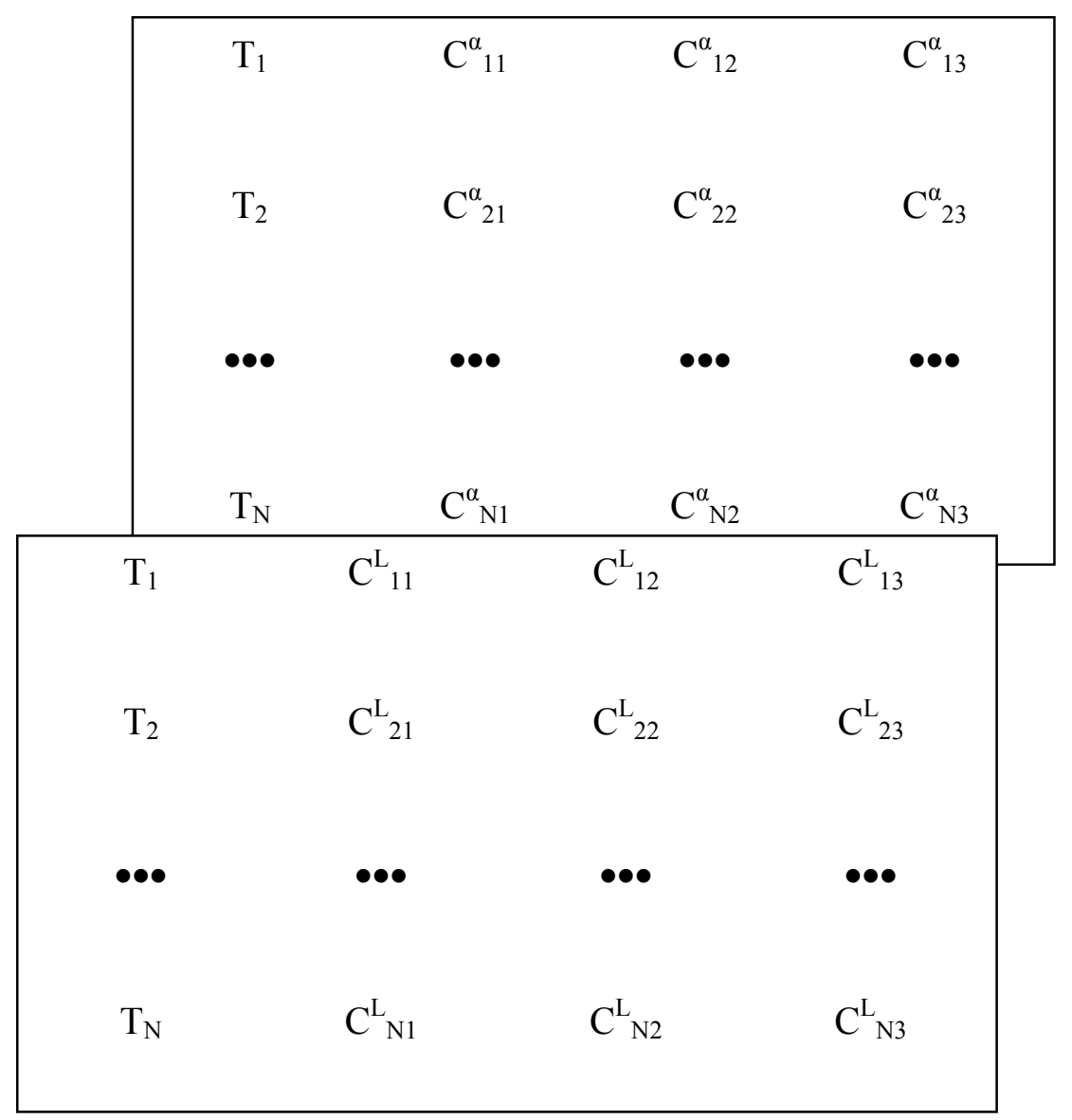

Figure 2.1-5. Storage scheme for tie line phase equilibrium data for the two-phase $\mathrm{L}+\alpha$ region in a quaternary system. The lower file represents storage of the temperature and composition end points for the $\alpha$-liquidus. The upper file represents storage of temperature and composition end points on the $\alpha$-solidus 


\section{Section 2: Solutionizing Prediction}

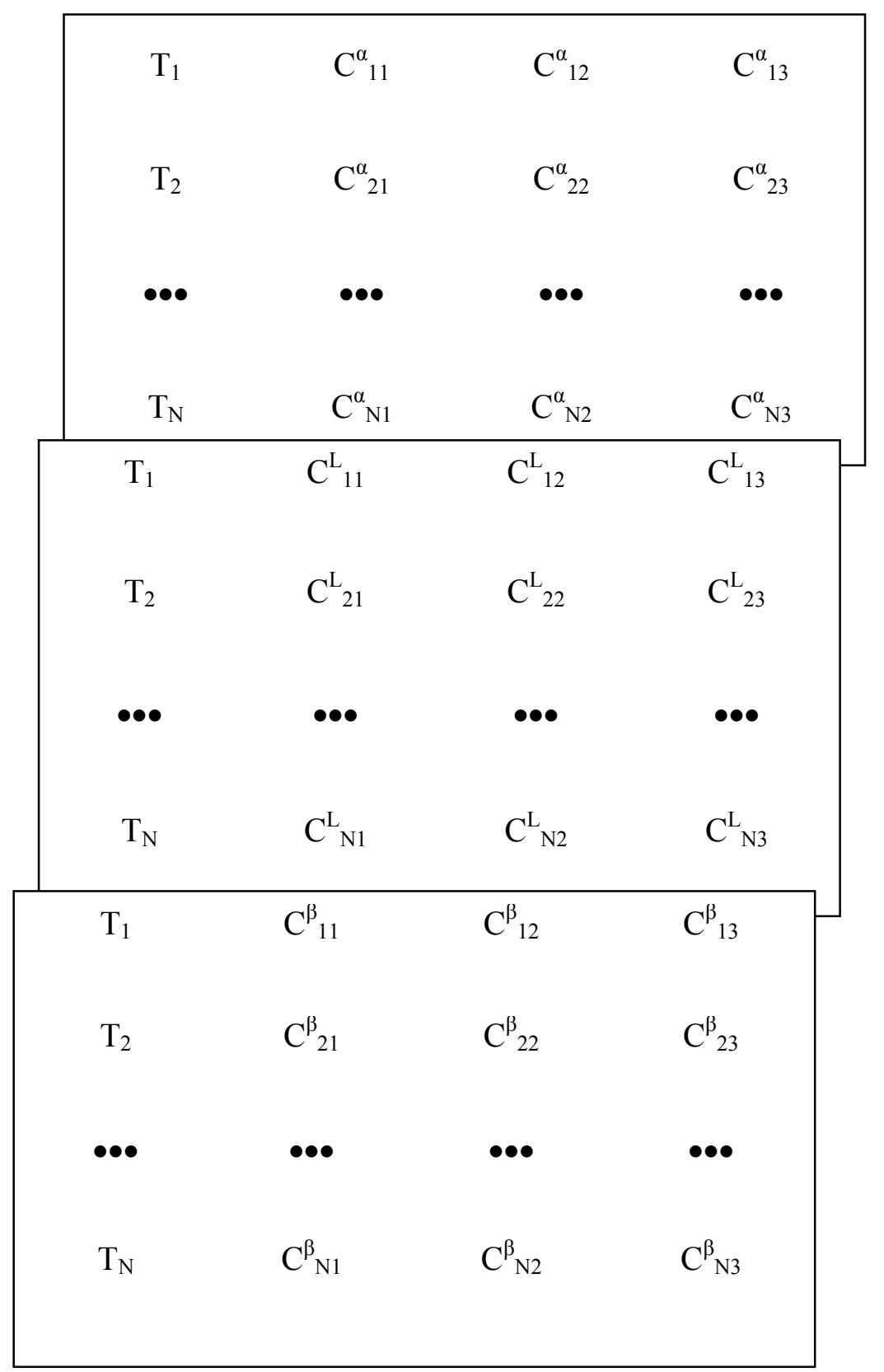

Figure 2.1-6. Storage scheme for tie triangles phase equilibrium data for the three-phase L + $\alpha+\beta$ region in a quaternary system. The upper file represents storage of the temperature and composition end points for the $\alpha$-phase, the center file for the liquid, and the lower file for the $\beta$-phase 


\section{Section 2: Solutionizing Prediction}

\subsection{DIFFCALC}

The functions of DIFFCALC are to organize, access, and calculate diffusion coefficients that are needed to simulate the kinetic behavior of multicomponent industrial aluminum alloys during casting and heat treatment. Diffusivity data, that have been collected from the literature or has been measured in conjunction with this DOE program, are organized and stored in files specific to the relevant casting alloys. During the solidification and heat treatment simulation procedures, DIFFCALC accesses the relevant diffusivity data and computes the diffusion coefficients appropriate to the currently simulated conditions of temperature and compositions.

The diffusion coefficients are represented by the symbol $D_{i j}^{\phi}$ in which the superscript $\phi$ refers to the phase, while $i$ and $j$ refer to solute alloying elements. In principle there are $(n-1)^{2}$ diffusion coefficients for an n-component alloy at each temperature. The first subscript, i, refers to the diffusing species. The second subscript, $\mathrm{j}$, refers to element whose composition gradient contributes to the driving force for diffusion. As illustration, Ficks first and second laws for diffusion of copper in the $\alpha$-phase of an Al-Si-Cu-Mg alloy could be written

$$
\begin{gathered}
-\frac{1}{\rho^{\alpha} A} \frac{\partial n}{\partial t}=D_{C u C u}^{\alpha} \frac{\partial C_{C u}^{\alpha}}{\partial x}+D_{C u S i}^{\alpha} \frac{\partial C_{S i}^{\alpha}}{\partial x}+D_{C u M g}^{\alpha} \frac{\partial C_{M g}^{\alpha}}{\partial x} \\
\frac{\partial C_{C u}^{\alpha}}{\partial t}=\frac{\partial}{\partial x}\left[D_{C u C u}^{\alpha} \frac{\partial C_{C u}^{\alpha}}{\partial x}+D_{C u S i}^{\alpha} \frac{d C_{S i}^{\alpha}}{d x}+D_{C u M g}^{\alpha} \frac{d C_{M g}^{\alpha}}{d x}\right]
\end{gathered}
$$

where $\rho^{\alpha}$ is the density of the alpha-phase, $\mathrm{A}$ is the cross sectional area normal to the $\mathrm{x}$-direction, $\mathrm{t}$ is time, and $D_{C u C u}^{\alpha}$ refers to the diffusion of $\mathrm{Cu}$ due to its own composition gradient, $D_{C u S i}^{\alpha}$ refers to the diffusion of $\mathrm{Cu}$ due to the composition gradient of silicon, $D_{\mathrm{CuMg}}^{\alpha}$ refers to the diffusion of $\mathrm{Cu}$ due to the composition gradient of magnesium.

Diffusivities for a particular temperature and composition are calculated from the equation: 


\section{Section 2: Solutionizing Prediction}

$$
D_{i j}^{\phi}=D_{O i j}^{\phi} \exp \left(-Q_{i j}^{\phi} / R T\right)
$$

in which $\mathrm{R}$ is the gas constant and $\mathrm{T}$ is absolute temperature. Both $D_{O i j}^{\alpha}$ and $Q_{i j}^{\phi}$ are polynomial functions of temperature and composition that have been determined from input data.

\section{$\underline{\text { 2.2.1 Diffusivities in Single Phase Regions }}$}

Data have been collected for binary eutectic and ternary eutectic systems, using Al-Cu, Al-Si, and $\mathrm{Al}-\mathrm{Si}-\mathrm{Cu}$ as the model systems. See section 3.5. For these systems, the concentrations of solutes in the terminal solid solution $\alpha$-phase are so dilute that the off-diagonal coefficients are negligible (i.e. $D_{i j}^{\phi} \cong 0$ for $i \neq j$ ) and the concentration dependence of the on-diagonal coefficients is negligible as well. For diffusion in single-phase regions of the Al-rich terminal solid solution the $\mathrm{D}_{\mathrm{Oii}}$ and $\mathrm{Q}_{\mathrm{ii}}$ values are stored for $\mathrm{Si}, \mathrm{Cu}$, and $\mathrm{Mg}$. Also, the presence of a small amount of magnesium has a significant influence on the diffusivity of copper. $\mathrm{D}_{\mathrm{CuCu}}$ is increased by a small concentration of $\mathrm{Mg}$ in the $\alpha$-phase. $\mathrm{D}_{\mathrm{CuMg}}$ still is negligible.

\section{$\underline{\text { 2.2.2 Diffusivities in Multi-Phase Regions }}$}

The secondary particles that form binary, ternary, and higher order microconstituent mixtures have very narrow composition ranges, negligible solubilities, and/or form in small volume fractions. Diffusion through individual constituent particles is not considered. Diffusion is considered to occur effectively through the primary solid solution $\alpha$-phase. The cross sectional area for diffusion in the $\alpha$-phase is decreased in proportion to the volume fraction of constituent particles in the local region. Importantly, measurements made in this program (section 3.5) indicate that the effective diffusivities of $\mathrm{Cu}$ and $\mathrm{Si}$ through binary eutectic $\alpha$-phase + silicon regions are 5 to 20 times the diffusivity through single-phase $\alpha$-regions. Effective diffusivity multipliers are stored in the data files and applied to the computations of diffusivity in multiphase regions. 


\section{Section 2: Solutionizing Prediction}

\subsection{CASTSEG}

The major objective of the module CASTSEG is to quantitatively describe the as-cast microstructure (phase amounts, morphologies, and microsegregation) of commercial multicomponent aluminum alloys. Data to specify solidification parameters, such as local solidification time and post solidification cooling rate, are imported from commercial solidification packages, from experimental databases, or computed through solidification simulation, if necessary. CASTSEG simulates solute redistribution during solidification and during post-solidification cooling to obtain a valid starting point that is representative of the specific casting parameters for heat treatment simulation by CASTSOLN.

For aluminum base casting alloys, including in particular Al-319 and Al-356, the features of the microstructure to be simulated are the fractions of primary aluminum rich phase $(\alpha)$, the fraction of silicon phase, the fraction of the copper-rich intermetallic compound $\mathrm{CuAl}_{2}(\theta)$, the fraction of magnesium-rich intermetallic phases, Q-phase and/or $\mathrm{Mg}_{2} \mathrm{Si}$, and the composition of other intermetallic compounds (especially the iron-rich intermetallics), and the distribution of the alloying elements $\mathrm{Cu}, \mathrm{Mg}$, and Si that have significant solubility within the primary aluminum $\alpha$-phase. CASTSEG has been implemented for binary, ternary, and quaternary systems, including Al-Si, Al-Cu, Al-Si-Cu, Al-Si-Mg, and Al-Si-Cu-Mg.

A representative microstructure for an $\mathrm{Al}-7 \% \mathrm{Si}-3.5 \% \mathrm{Cu}$ alloy is shown in Figure 2.3-1. Primary aluminum-rich $\alpha$-phase is the major constituent and has formed a dendritic microstructure. The silicon particles and the intermetallic $\theta$-phase are minor constituents that are found in the interdendritic regions.

The basic solidification models used and validated for binary alloys that were the starting point for development of the CASTSEG model are reviewed, briefly, below. The implementation of the solidification models by the CASTSEG software is described, including the extension of the implementation to ternary and higher order alloy systems. Typical results of the simulations for 


\section{Section 2: Solutionizing Prediction}

multicomponent aluminum alloys are presented. Then new features of the solidification models to handle the complexities involved in simulating solidification and solute redistribution in multicomponent-multiphase aluminum casting alloys, as uncovered in the database and validation tasks of this program (section 3.4-3.5), are described and typical results presented.

\section{$\underline{\text { 2.3.1 Solidification Models }}$}

2.3.1.1 Gulliver-Scheil Model Assumptions of the Gulliver-Scheil (G-S) model are

- No undercooling prior to nucleation or during growth of solid phases. Equilibrium is assumed at all interfaces.

- Mixing within the liquid phase between dendrite arms is complete.

- The overall concentration of solute within the volume element does not change.

- There is no solute transport, by diffusion, within the solid phases.

G-S is a limiting case. The predictions are independent of assumptions of solidification morphology, solidification kinetics, or casting parameters. When the G-S assumptions are applied to a characteristic volume representing a growing dendrite branch and the surrounding liquid, solute partitioning during solidification leads to the most extreme coring within the dendrite, the greatest fraction of low melting (eutectic) constituents, and the widest freezing range (concomitantly, the lowest incipient melting temperature). Applying the assumptions of the G-S model, the predictions for coring and secondary phase formation do not depend on cooling rate, dendrite spacing or dendrite morphology.

2.3.1.2 Limited Solid Diffusion Model In the limited solid diffusion (LSD) model the assumptions for solidification and solute redistribution within a characteristic dendritic volume element are the same as for the G-S model (including the assumption of local equilibrium) except for the last assumption listed, that of no solid-state diffusion. Instead the following is assumed.

- Diffusion of solute occurs within the solidifying primary phase dendrite. 


\section{Section 2: Solutionizing Prediction}

Diffusion within the primary phase dendrite reduces the extent of coring and decreases the fraction of low melting constituents toward the equilibrium amount. Unless solid diffusion is extensive, the incipient melting point is not raised. The G-S assumptions have been considered sufficiently accurate for heat transfer simulations of solidification in aluminum alloys, where the objective is to predict cooling rates, thermal gradients, residual stresses, and gross porosity. The G-S model is not a good predictor of the baseline microstructure for input to solution treatment simulations. The response of an aluminum alloy to solution treatment is sensitive to the concentration gradient (coring) within the primary phase and the fraction of low melting constituents in the as-cast microstructure. The LSD model is a good predictor of as-cast microstructure and solute distribution for binary alloys in which the primary phase is the major constituent and the secondary phase constituents is restricted to the interdendritic regions. The LSD model must be modified and extended to account for the complexities involved in multicomponent and multiphase solidification encountered in commercial aluminum casting alloys. Extensions to the LSD model are discussed below. First, the simplest model for dendrite geometry and growth rate is described. 


\section{Section 2: Solutionizing Prediction}

2.3.1.3 Characteristic Volume Element Analyses and simulations of microsegregation usually assume a volume element with a characteristic dimension comparable to the dendrite arm spacing (das). The geometry of the volume element has been taken to be plate-like, cylindrical, or spherical. When a 1-D volume element is desired, we assume a plate-like geometry for the volume element and the characteristic dimension of the volume element (L) is one-half the dendrite spacing $(L=$ das/2). We use the symbol $\lambda$ to represent position in the thickness direction within the volume element. One edge of the volume element, $\lambda=0$, is at the center of a dendrite arm, while the other edge, $\lambda=$ das $/ 2$, is at the center of the interdendritic region between two (or more) growing dendrite arms. The position of the solid liquid interface is represented by the symbol $\lambda *$.

The dendrite arm spacing is determined by the amount of time liquid and solid are in contact during solidification, i.e., the local solidification time $\left(\theta_{\mathrm{f}}\right)$. The empirical relation is of the form:

$$
d a s=\theta_{f}^{n}
$$

where $n$ is empirical constant, usually between one-third and one-half $(1 / 3<n<1 / 2)$.

During the process of solidification, the reduced variable $f_{S}=\lambda / L$ represents the fraction of the volume element that has solidified. The definitions of 1 and $f_{S}$ within a 1-D, plate-like solidification model are illustrated for a ternary an Al-Si-Cu alloy in Figure 2.3-2. After solidification, $f_{S}$ refers to the fraction of the solid within the characteristic volume element that has a composition below a stated value, i.e., the solid fraction enclosed by a stated isoconcentration surface.

2.3.1.4 Dendrite Thickening Rate Another assumption in dendrite solidification models concerns the rate of dendrite arm thickening. This is the rate at which the solidifying interface, $\lambda^{*}$, within the characteristic volume element moves from the center of the dendrite arm, $\lambda=0$, into the interdendritic liquid pool, toward $\lambda=$ das/2, which is halfway to the neighboring dendrite 


\section{Section 2: Solutionizing Prediction}

arm(s). In terms of the 1-D plate-like characteristic volume element used to represent dendrite morphology, the dendrite thickening rate may be expressed as $\Delta \lambda * / \Delta t$ or $\Delta f_{s}^{*} / \Delta t$, where the reduced variable $f_{S}=\lambda / L=2 \lambda /$ das and $f_{S} *=\lambda * / L$. Commonly, we assume a constant rate of thickening of the solidifying dendrite within the volume element and that invariant eutectic constituents form instantaneously, which yields for the dendrite thickening rate:

$$
\frac{\Delta f_{S}}{\Delta t}=-\frac{\Delta f_{L}}{\Delta t}=\frac{\left(1-f_{E}\right)}{\theta_{f}}
$$

The underlying assumptions that lead to a constant dendrite thickening rate are (a) that rate of heat extraction is constant in the locality of the characteristic volume element, (b) that the enthalpy of fusion is the same for each increment of solidification, and (c) that an invariant reaction occurs at a single isotherm that moves continuously in a solidifying casting. When CASTSEG is integrated with comprehensive solidification modeling software, computed solidification rates will be coupled with the solute redistribution simulation.

\subsubsection{CASTSEG Simulation Software}

The major routines and algorithms employed in CASTSEG are illustrated below, first for a binary alloy system, then for a ternary alloy system. The differences encountered in going from two to three components point out the relevant issues to be handled in simulating n-component systems with increasing degrees of freedom.

2.3.2.1 Binary Alloy Systems. The implementation of the solidification models by the CASTSEG module is summarized for a binary alloy system below.

\section{Input to the Solidification Simulation Routines}

1. Alloy system parameters: 


\section{Section 2: Solutionizing Prediction}

Phase relations in the solidification regime (Input is prepared by the PHASECALC module.):

- For binary alloys the liquidus, solidus, and solyus may be entered as a series of straight line segments or as curved lines:

$$
T^{\phi}=f\left(C^{\phi}\right)
$$

where $\mathrm{T}$ is temperature, $\mathrm{C}$ is solute concentration (wt \%), and the superscript $\phi$ denotes the phase. To represent a curved linear phase boundary we use a series of short straight-line segments (Figure 2.1-2).

- Diffusion coefficients for the alloying elements (Input is prepared by the DIFFCALC module):

The diffusion coefficient is entered as a function of temperature and composition, typically,

$$
D^{\phi}=D_{O}^{\phi} \exp \left(-Q^{\phi} / R T\right)
$$

For a binary alloy the pre-exponential constant may be a function of solute content $D_{O}^{\phi}=f\left(C^{\phi}\right)$ and, similarly, the activation energy $Q^{\phi}=f\left(C^{\phi}\right)$.

2. Dendrite solidification model parameters:

- Relation between dendrite arm spacing and local solidification time.

Dendrite arm spacing (das) and local solidification time $\left(\theta_{\mathrm{f}}\right)$ may be entered as characteristic parameters of the casting process, if measured or if predicted by a heat transfer simulation of the casting process. If only one, das or $\theta_{\mathrm{f}}$, is known, the other may be obtained from an empirical relation; such as equation 2.3-1.

- Geometry and an expression for the thickening rate of dendrite arms.

The default assumption for geometry of dendrites, and thus the characteristic volume element for the simulation is $1-\mathrm{D}$, plate-like with a thickness $=$ das $/ 2$. The default 


\section{Section 2: Solutionizing Prediction}

dendrite thickening rate is governed by a constant heat extraction rate as given in equation 2.3-2.

3. Alloy and Casting Process Parameters:

- Solute concentration in the initial melt:

$$
\mathrm{C}=\mathrm{C}_{0}
$$

4. Computation Control Parameters and Numbering of Distance Elements $(\Delta \lambda)$ :

- The characteristic volume element

The characteristic volume element of thickness das/2 is divided into $\mathrm{N}$ distance elements or "slices" (typically, $200<\mathrm{N}<1000$ ). For uniformly spaced elements

$$
\Delta \lambda=\operatorname{das} / 2 \mathrm{~N}
$$

The slice centered at $\lambda=0$ is numbered $\mathrm{n}=0$ and the slice centered at $\lambda=$ das $/ 2$ is numbered $n=N$. Half of slice $n=0$ and $n=N$ is in the characteristic volume element and half of each of these slices is in a neighboring volume element. Slices labeled $\mathrm{n}=\tilde{-}-1$, centered at $\lambda \stackrel{=}{=}-\Delta \lambda$, and $\mathrm{n}=\mathrm{N}+1$, centered at $\lambda=$ das $/ 2+\Delta \lambda$, are used to implement the symmetry boundary condition.

- The time to solidify an amount $\Delta \lambda$

The time step for solidification, $\Delta \theta_{\mathrm{S}}$ is the time to solidify an amount $\Delta \lambda$. Using equation 2.3-2,

$$
\Delta \theta_{S}=\frac{\theta_{f}}{N\left(1-f_{E}\right)} \frac{2}{d a s}
$$

- The time step for the diffusion computation

Typically a time step for the diffusion computation, $\Delta \mathrm{t}_{\text {Diff, }}$, is selected so that

$$
\frac{D^{\phi} \Delta t_{\text {Diff }}}{\Delta \lambda^{2}} \leq \frac{1}{8}
$$




\section{Section 2: Solutionizing Prediction}

The diffusion time step $\left(\Delta t_{\text {Diff }}\right)$ typically is smaller than the solidification time step $\left(\Delta \theta_{S}\right)$ and the program iterates through the FDM diffusion calculations several times for each iteration of the solidification calculation. The upper limit on the diffusion time step is the solidification time step $\Delta t_{D i f f} \leq \Delta \theta_{S}$.

\section{$\underline{\text { Solidification Simulation Steps }}$}

1. Starting with the initial liquid concentration $\mathrm{C}_{0}$ and then with each newly computed liquid composition $\mathrm{C}^{\mathrm{L}}$, locate the corresponding liquidus temperature $\mathrm{T}^{\mathrm{L}}$, which is the temperature of the local volume element and, in particular, the temperature of the solid/liquid interface. See section 2.1 for procedure used by PHASECALC to find $\mathrm{T}^{\mathrm{L}}$. The finite difference element at the solid/liquid interface is labeled $n^{*}$.

2. From the tie line for temperature $\mathrm{T}^{\mathrm{L}}$, find the composition of the solid $C_{n^{*}}^{\alpha}$ in equilibrium with the liquid $\mathrm{C}^{\mathrm{L}}$.

3. Simulate the solidification of slice $n^{*}$ to transform from liquid of concentration $\mathrm{C}^{\mathrm{L}}$ to solid of concentration $C_{n^{*}}^{\alpha}$.

4. Compute diffusion in the solid phase, in elements $n<n^{*}$, according to Fick's Second Law in FDM form:

$$
\frac{\Delta C_{n}^{\alpha}}{\Delta t}=\frac{\Delta}{\Delta x}\left(D\left(C_{n}^{\alpha}, T\right)\left(\frac{\Delta C^{\alpha}}{\Delta x}\right)_{n}\right)
$$

with the boundary conditions

$$
\begin{gathered}
\text { @ } \lambda=0 \quad(n=0) ; \quad\left(\frac{\Delta C^{\alpha}}{\Delta x}\right)_{n=0}=0 \\
\text { @ } \lambda=\lambda^{*} \quad\left(n=n^{*}\right) ; \quad C_{n^{*}}^{\alpha}=C_{n^{*}}^{\alpha}\left(\text { fixed during time step, } \Delta \theta_{S}\right)
\end{gathered}
$$

5. Implement the FDM to iteratively evaluate equation 2.3-5.

6. Recompute the composition of the remaining liquid, $\mathrm{C}^{\mathrm{L}}$, from the materials balance 


\section{Section 2: Solutionizing Prediction}

$$
\sum_{n=0}^{n^{*}} C_{n}^{\alpha}(n, t) \Delta f_{S}+C^{L}\left(1-f_{S}\right)=C_{O}
$$

where $\mathrm{C}^{\mathrm{L}}$ is the only unknown parameter.

5. Compare the new value of $\mathrm{C}^{\mathrm{L}}$ to the eutectic composition $\left(\mathrm{C}_{\mathrm{E}}\right)$.

- If $\mathrm{C}^{\mathrm{L}}<\mathrm{C}_{\mathrm{E}}$, increment $\mathrm{n}^{*}$ by 1 , increment time (t) by $\Delta \theta_{\mathrm{S}}$, and return to step 1 .

- If $\mathrm{C}^{\mathrm{L}}>\mathrm{C}_{\mathrm{E}}$, simulation of solidification is completed. The remaining liquid solidifies as the two-phase eutectic constituent at the compositions given by the eutectic tie line and the fractions given by the lever rule.

6. Check the program control indices.

- If indicated by the user, simulate post-solidification cooling over a specified cooling curve from the eutectic temperature to a specified temperature where diffusion becomes negligible. Then prepare output.

- Else, the solute distribution and phase fractions computed are ready for output from CASTSEG and input to CASTSOLN.

\section{Output in Tabular or Graphical Form}

1. Solidification curve - fraction solid formed versus temperature in the freezing range.

2. Solute profile - as example, distribution of the alloying element in the alpha-phase across the characteristic volume element $\left(\mathrm{C}^{\alpha}\right.$ vs $\mathrm{f}^{\alpha}=21 /$ das$)$ during solidification, at the end of solidification, and after post-solidification cooling.

3. Data file - A file of compositions, phase fractions, dendrite spacing, and overall composition for input to CASTSOLN. 


\section{Section 2: Solutionizing Prediction}

2.3.2.2 Ternary Alloy Systems The implementation of the solidification models by the CASTSEG module is summarized for a ternary alloy system below.

\section{$\underline{\text { Input to the Solidification Simulation Routines }}$}

1. Alloy System parameters:

- Phase relations from PHASECALC

The liquidus, solidus, and solvus surfaces bound two-phase regions (e.g. the primary solidification range, $L+\alpha$ ) and are given by:

$$
\mathrm{T}^{\phi}=\mathrm{f}\left(\mathrm{C}_{1}{ }^{\phi}, \mathrm{C}_{2}{ }^{\phi}\right)
$$

where the subscripts, 1 and 2, refer to concentrations of the two alloying elements and the superscript, $\phi$, stands for the phase.. If the surface is curved, PHASECALC represents it as a series of flat triangular plane segments (Figure 2.1-4).

Three-phase regions (e.g. where liquid transforms to two solid phases) are represented by three lines of two-fold saturation, one for each phase. If the lines are curved, they are represented by a series of straight-line segments.

- Diffusion coefficients from DIFFCALC:

Diffusion coefficients for a given temperature are given by the matrix:

$$
\left[D^{\phi}\right]=\left[\begin{array}{cc}
D_{11}^{\phi} & D_{12}^{\phi} \\
D_{21}^{\phi} & D_{22}^{\phi}
\end{array}\right]
$$

in which $\mathrm{D}^{\phi}{ }_{\mathrm{ij}}=\mathrm{f}\left(\mathrm{T}, \mathrm{C}_{1}{ }^{\phi}, \mathrm{C}_{2}{ }^{\phi}\right)$. The first subscript of the diffusion coefficient represents the diffusing alloy element and the second subscript represents the alloying element whose concentration gradient influences that component of the diffusion flux. The matrix must be evaluated for each time step and for each solid element.

Diffusivity measurements for the $\alpha$-phase, the terminal solid solution of aluminum with $\mathrm{Si}$, $\mathrm{Cu}$, and $\mathrm{Mg}$, have shown that the off-diagonal coefficients $\left(\mathrm{D}^{\alpha}{ }_{\mathrm{CuSi}}, \mathrm{D}^{\alpha}{ }_{\mathrm{CuMg}}, \mathrm{D}^{\alpha}{ }_{\mathrm{SiCu}}, \mathrm{D}^{\alpha}{ }_{\mathrm{SiMg}}\right.$, $\mathrm{D}^{\alpha}{ }_{\mathrm{MgCu}}$, and $\left.\mathrm{D}^{\alpha}{ }_{\mathrm{MgSi}}\right)$ can be neglected and the dependence of the diagonal coefficients $\left(\mathrm{D}^{\alpha}{ }_{\mathrm{CuCu}}, \mathrm{D}^{\alpha}{ }_{\mathrm{MgMg}}\right.$, and $\mathrm{D}^{\alpha}{ }_{\mathrm{SiSi}}$ ) on composition can be neglected. (Note section 3.5.) The 


\section{Section 2: Solutionizing Prediction}

required data are the pre-exponential coefficient and the activation energy for the solute in the $\alpha$-phase: $D_{O-C u C u}^{\alpha}, Q_{C u C u}^{\alpha}, D_{O-S i S i}^{\alpha}, Q_{S i S i}^{\alpha}, D_{O-M g M g}^{\alpha}$, and/or $Q_{M g M g}^{\alpha}$.

The diffusion of $\mathrm{Cu}, D_{\mathrm{CuCv}}^{\alpha}$ is enhanced by the presence of $\mathrm{Mg}$. Diffusivity in multiphase regions is simulated by an effective diffusion coefficient for the $\alpha$-phase..

2. Dendrite Solidification Model Parameters:

- Relation between dendrite arm spacing and local solidification time.

The same as for a binary system.

3. Geometry and an expression for the thickening rate of dendrite arms.

The same as for a binary system.

4. Alloy and Casting Process Parameters:

- Solute concentrations of alloying elements in the initial melt.

$$
\mathrm{C}_{1}=\mathrm{C}_{1}{ }^{0} \text { and } \mathrm{C}_{2}=\mathrm{C}_{2}{ }^{0}
$$

- Local solidification time and an expression for the cooling rate after solidification.

The same as for a binary system.

5. Computation Control Parameters:

- Thickness and numbering of distance elements $(\Delta \mathrm{x})$.

The same as for binary systems.

- Size of time steps $(\Delta t)$ for FDM computation.

The same as for binary systems.

\section{$\underline{\text { Solidification Simulation Steps - Primary Phase Solidification; } \mathrm{L} \rightarrow \alpha}$}

1. Starting with the initial liquid composition $\mathrm{C}_{1}{ }^{0}$ and $\mathrm{C}_{2}{ }^{0}$ and then for each newly computed pair of solute concentrations in the liquid, $\mathrm{C}_{1}{ }^{\mathrm{L}}$ and $\mathrm{C}_{2}{ }^{\mathrm{L}}$, locate on the liquidus surface the corresponding temperature $\mathrm{T}^{\mathrm{L}}$, which is the temperature of the local volume element and, in 


\section{Section 2: Solutionizing Prediction}

particular, the temperature of the solid/liquid interface. See section 2.1 for procedure used by PHASECALC to find $\mathrm{T}^{\mathrm{L}}$ in the two-phase region of a ternary alloy.

2. From the tie line for the liquidus point $\left(\mathrm{T}^{\mathrm{L}}, \mathrm{C}_{1}{ }^{\mathrm{L}}, \mathrm{C}_{2}{ }^{\mathrm{L}}\right)$, find the composition of the solid phase, $\mathrm{C}_{1}{ }^{\alpha}$ and $\mathrm{C}_{2}{ }^{\alpha}$, in equilibrium with the liquid.

3. Simulate the solidification of slice $\mathrm{n}^{*}$ to transform from liquid of composition $\mathrm{C}_{1}^{\mathrm{L}}, \mathrm{C}_{2}{ }^{\mathrm{L}}$ to solid of composition $\mathrm{C}_{1}{ }^{\alpha}, \mathrm{C}_{2}{ }^{\alpha}$.

4. Compute diffusion in the primary solid phase, in elements $n<n^{*}$, according to Fick's Second Law in FDM form:

$$
\left[\begin{array}{l}
\left(\Delta C_{1 n}^{\alpha} / \Delta t\right)_{n} \\
\left(\Delta C_{2 n}^{\alpha} / \Delta t\right)_{n}
\end{array}\right]=\left[\begin{array}{ll}
D_{11}^{\alpha}\left(T, C_{1 n}^{\alpha}, C_{2 n}^{\alpha}\right) & D_{12}^{\alpha}\left(T, C_{1 n}^{\alpha}, C_{2 n}^{\alpha}\right) \\
D_{21}^{\alpha}\left(T, C_{1 n}^{\alpha}, C_{2 n}^{\alpha}\right) & D_{22}^{\alpha}\left(T, C_{1 n}^{\alpha}, C_{2 n}^{\alpha}\right)
\end{array}\right]\left[\begin{array}{l}
\left(\Delta^{2} C_{1 n}^{\alpha} / \Delta x^{2}\right)_{n} \\
\left(\Delta^{2} C_{2 n}^{\alpha} / \Delta x^{2}\right)_{n}
\end{array}\right]
$$

which simplifies for the limited solubility range $\alpha$-phase.

$$
\left[\begin{array}{l}
\left(\Delta C_{1 n}^{\alpha} / \Delta t\right)_{n} \\
\left(\Delta C_{2 n}^{\alpha} / \Delta\right)_{n}
\end{array}\right]=\left[\begin{array}{cc}
D_{11}^{\alpha}(T) & 0 \\
0 & D_{22}^{\alpha}(T)
\end{array}\right]\left[\begin{array}{l}
\left(\Delta^{2} C_{1 n}^{\alpha} / \Delta x^{2}\right)_{n} \\
\left(\Delta^{2} C_{2 n}^{\alpha} / \Delta x^{2}\right)_{n}
\end{array}\right]
$$

with the boundary conditions

$$
\begin{gathered}
\text { @ } \lambda=0 \quad(n=0) ; \quad\left(\frac{\Delta C_{1}^{\alpha}}{\Delta x}\right)_{n=0}=0\left(\frac{\Delta C_{2}^{\alpha}}{\Delta x}\right)_{n=0}=0 \\
\text { @ } \left.\lambda=\lambda^{*} \quad\left(n=n^{*}\right) ; \quad C_{1 n^{*}}^{\alpha}=C_{1 n^{*}}^{\alpha} C_{2 n^{*}}^{\alpha}=C_{2 n^{*}}^{\alpha} \text { (fixed during time step, } \Delta \theta_{S}\right)
\end{gathered}
$$

5. Recompute the composition of the remaining liquid, $\mathrm{C}_{1}{ }^{\mathrm{L}}$ and $\mathrm{C}_{2}{ }^{\mathrm{L}}$, from the individual solute balances

$$
\begin{aligned}
& \sum_{n=0}^{n^{*}} C_{1 n}^{\alpha}(n, t) \Delta f_{S}+C_{1}^{L}\left(1-f_{S}\right)=C_{1 O} \\
& \sum_{n=0}^{n^{*}} C_{2 n}^{\alpha}(n, t) \Delta f_{S}+C_{2}^{L}\left(1-f_{S}\right)=C_{2 O}
\end{aligned}
$$

where $\mathrm{C}_{1}{ }^{\mathrm{L}}$ and $\mathrm{C}_{2}^{\mathrm{L}}$ are the only unknown parameters. 


\section{Section 2: Solutionizing Prediction}

6. Check if the new values of $\mathrm{C}_{1}{ }^{\mathrm{L}}$ and $\mathrm{C}_{2}{ }^{\mathrm{L}}$ are beyond the primary phase solidification range for this alloy system.

- If no, increment $n^{*}$ by 1 , increment time by $\Delta \theta_{\mathrm{S}}$, and return to step 1 .

- If yes, begin simulation of the solidification of two solid phases.

$\underline{\text { Solidification Simulation Steps - Primary and Secondary Phase Solidification; } L \rightarrow \alpha .+\beta}$.

1. For each newly computed pair of liquid concentrations $\mathrm{C}_{1}{ }^{\mathrm{L}}$ and $\mathrm{C}_{2}{ }^{\mathrm{L}}$ locate the composition on the line of two fold saturation (eutectic valley). Find the corresponding temperature $\mathrm{T}^{\mathrm{L}}$, which is the temperature of the local volume element and, in particular, the temperature of the solid/liquid interface. The finite difference element at the solid/liquid interface is labeled $n^{*}$. See section 2.1 for procedure used by PHASECALC to find $T^{L}$ in the three-phase region of a ternary alloy.

2. From the tie lines corresponding to the liquidus point $\left(\mathrm{T}^{\mathrm{L}}, \mathrm{C}_{1}{ }^{\mathrm{L}}, \mathrm{C}_{2}{ }^{\mathrm{L}}\right)$ find the composition of the solid phases $\mathrm{C}_{1}{ }^{\alpha}, \mathrm{C}_{2}{ }^{\alpha}$ and $\mathrm{C}_{1}{ }^{\beta}, \mathrm{C}_{2}{ }^{\beta}$ in equilibrium with the liquid.

3. For each slice $\mathrm{n}<\mathrm{n}^{*}$ (at time $\mathrm{t}$ ) determine if its average composition, $\bar{C}_{1 n}$ and $\bar{C}_{2 n}$, is above the solubility limit at $\mathrm{T}^{\mathrm{L}}$ for the primary phase.

- If the slice is single phase, set $C_{1 n}^{\alpha}=\bar{C}_{1 n}$, set $C_{2 n}^{\alpha}=\bar{C}_{2 n}$, and $f_{n}^{\alpha}=1$.

- If the slice is two-phase, determine the solubility limits, $\mathrm{C}_{1}{ }^{\alpha *}, \mathrm{C}_{2}{ }^{\alpha *}$, and $\mathrm{C}_{1}{ }^{\beta_{*}}, \mathrm{C}_{2}{ }^{\beta_{*}}$, for this temperature $\mathrm{T}^{\mathrm{L}}$. From the average composition and the compositions of the individual phases compute the fraction of primary phase, $\mathrm{f}_{\mathrm{n}}{ }^{\alpha}$, within the slice. Set $\mathrm{C}_{1 \mathrm{n}}{ }^{\alpha}=\mathrm{C}_{1}{ }^{\alpha *}$, and $\mathrm{C}_{2 \mathrm{n}}{ }^{\alpha}=\mathrm{C}_{2}{ }^{\alpha}$.

4. Compute diffusion in the primary solid phase, in elements $n<n^{*}$, according to Fick's Second Law using equations 2.3-8.

5. Recompute the composition of the remaining liquid, $\mathrm{C}_{\mathrm{L}}{ }^{*}$, from the following four relations: 


\section{Section 2: Solutionizing Prediction}

$$
\begin{gathered}
\sum_{n=0}^{n^{*}}\left[C_{1 n}^{\alpha} f_{n}^{\alpha}+C_{1 n}^{\beta} f_{n}^{\beta}\right] \Delta f_{S}+C_{1}^{L}\left(1-f_{S}^{*}\right)=C_{1 O} \\
\sum_{n=0}^{n^{*}}\left[C_{21 n}^{\alpha} f_{n}^{\alpha}+C_{2 n}^{\beta} f_{n}^{\beta}\right] \Delta f_{S}+C_{2}^{L}\left(1-f_{S}^{*}\right)=C_{2 O} \\
C_{1}^{L^{*}}=m C_{2 j}^{L^{*}}+b \\
f_{n^{*}}^{\alpha}+f_{n^{*}}^{\beta}=1
\end{gathered}
$$

where $\mathrm{C}_{1}^{\mathrm{L}}, \mathrm{C}_{2}^{\mathrm{L}}, \mathrm{f}_{\mathrm{n}^{*}}{ }^{\alpha}$, and $\mathrm{f}_{\mathrm{n}^{*}}{ }^{\beta}$ are the only unknown parameters. The first two equations are solute balances. The third equation is the projection of the eutectic valley on the basal plane ( $m$ and $b$ are constants). Satisfying this relation constrains $C_{1}{ }^{L}$ and $C_{2}{ }^{L}$ to fall on the eutectic valley line.

6. Check if the new values of $\mathrm{C}_{1}{ }^{\mathrm{L}}$ and $\mathrm{C}_{2}{ }^{\mathrm{L}}$ have reached the ternary eutectic composition for this alloy system.

- If no, increment $\mathrm{n}^{*}$ by 1 and return to step 1 .

- If yes, simulate solidification of the remaining liquid as the ternary eutectic mixture of three solid phases. The solute distribution and phase fractions are now computed.

\section{Output in Tabular or Graphical Form}

- Solidification curve - fraction solid formed versus temperature in the freezing range.

- Solute profile - distribution of alloying elements across the characteristic volume element $\left(\mathrm{C}_{1}{ }^{\phi}\right.$ and $\mathrm{C}_{2}{ }^{\phi}$ vs $\lambda=2 \lambda /$ das $\}$ during solidification and at the end of solidification.

\subsubsection{Application of CASTSEG to Al-6.5\% $\mathrm{Si-3.5 \% \textrm {Cu }}$}




\section{Section 2: Solutionizing Prediction}

The major alloying elements for the Al-319 family of alloys are silicon and copper and the $\mathrm{Al}-\mathrm{Si}-\mathrm{Cu}$ ternary system represents the major solidification characteristics of Al-319 alloys. Consideration of a ternary $\mathrm{Al}-6.5 \% \mathrm{Si}-3.5 \% \mathrm{Cu}$ alloy brings out many of the features of multicomponent and multiphase solidification and solution treatment processes that require modifications to the basic models that have successfully modeled binary alloys. Application of the extended models to a quaternary alloy will be presented in the next section.

The features of the microstructure to be simulated are the fractions of primary aluminum-rich phase $(\alpha)$, the fraction of silicon-phase, the fraction of the intermetallic compound $\mathrm{CuAl}_{2}(\theta)$, and the distribution of the alloying elements $\mathrm{Cu}$ and Si dissolved within the primary aluminum phase. Figure 2.3-1 shows a typical microstructure for $\mathrm{Al}-6.5 \% \mathrm{Si}-3.5 \% \mathrm{Cu}$ alloy that is not grain refined and is not modified. The solidification path for this alloy would be for primary phase dendrites of $\alpha$-phase to solidify first from the melt, followed by simultaneous solidification of the $\alpha$-phase and the silicon-phase. Finally, at the ternary eutectic temperature $\alpha$-phase, silicon-phase, and $\theta$-phases solidify simultaneously.

The characteristic volume element is illustrated in Figure 2.3-2 for the solidification of an Al-Si-Cu alloy when the local temperature is in the $L \rightarrow \alpha+$ silicon three-phase region. The characteristic dimension of the volume element $(L)$ is one-half the dendrite spacing $(L=$ das $/ 2)$. In Figure 2.3-2 we use $\lambda$ to represent position in the thickness direction within the volume element. One edge of the volume element, $\lambda=0$, is considered to be at the center of a dendrite arm. The other edge of the volume element $\lambda=d a s / 2$ is considered to be at the center of the interdendritic region between two (or more) growing dendrite arms. The position of the solid liquid interface is represented by the symbol $\lambda^{*}$.

2.3.3.1 Gulliver-Scheil Model The results of numerical simulation for the solidification of $\mathrm{Al}-6.5 \% \mathrm{Si}-3.5 \% \mathrm{Cu}$ alloy, using the assumptions of the G-S model (section 2.3.1.1), are presented in Figures 2.3-3 and 2.3-4. The fractions of $\alpha$-phase, silicon-phase, and $\theta$-phase solidified as functions of temperature, the solidification curve, are plotted in Figure 2.3-3. The 


\section{Section 2: Solutionizing Prediction}

distributions of the major alloying elements, $\mathrm{Si}$ and $\mathrm{Cu}$, in the $\alpha$-phase across a characteristic dendrite element at the end of solidification (i.e. just below the ternary eutectic temperature) are plotted in Figure 2.3-4. Primary $\alpha$-phase forms from the liquidus temperature until the line of two-fold saturation is reached. At the point the composition of the liquid reaches the line of two-fold saturation (eutectic valley), the percentage of $\alpha$-phase at the dendrite core is computed by the G-S model to be $43 \%$. The solute concentrations in the $\alpha$-phase at the dendrite increase from $0.75 \% \mathrm{Si}$ and $0.5 \% \mathrm{Cu}$ at the center of the dendrite to $1.1 \% \mathrm{Si}$ and $0.9 \% \mathrm{Cu}$ at the $\alpha / \mathrm{L}$ interface at the point the alloy reaches the eutectic valley. Simultaneous solidification of $\alpha$-phase and silicon-phase begins as the alloy cools along the line of two-fold saturation. The $\mathrm{Cu}$ concentration of the $\alpha$-phase continues to increase and the Si concentration in the $\alpha$-phase decreases slightly. Only about $8 \%$ liquid remains as the ternary eutectic temperature is reached. The remaining liquid transforms to the eutectic mixture of $\alpha+$ silicon $+\theta$.

As the alloy cooled along the eutectic valley, through the $L \rightarrow \alpha+$ silicon region, the solubility of $\mathrm{Si}$ in the $\alpha$-phase decreases and there would be a potential for silicon-phase to precipitate. If the G-S assumption of no undercooling before nucleation were extended to solid-state precipitation, fine silicon-phase particles would form in the silicon-rich regions of the dendrite arm that solidified as cored single $\alpha$-phase. About $18 \%$ of the cored $\alpha$-phase at the center of the dendrite would remain single phase at the end of solidification. In the region that solidified as the binary eutectic mixture of $\alpha$-phase and silicon-phase, it would be reasonable that no new silicon particles would precipitate. As the solubility of silicon in the $\alpha$-phase decreased, the existing silicon-phase particles would thicken.

2.3.3.2 Limited Solid Diffusion Model The results of numerical simulation for the solidification of $\mathrm{Al}-6.5 \% \mathrm{Si}-3.5 \% \mathrm{Cu}$ alloy, using the assumptions of the LSD model (section 2.3.1.2), are presented in Figure 2.3-5 and 2.3-6. The solidification curve for the LSD model is nearly identical to the solidification curve for the G-S model, Figure 2.3-3, and is not shown. 


\section{Section 2: Solutionizing Prediction}

The $\mathrm{Si}$ and $\mathrm{Cu}$ distributions within the $\alpha$-phase are shown in Figure 2.3-5. The distributions of $\mathrm{Cu}$ in the $\alpha$-phase for the LSD and G-S models are compared in Figure 2.3-6.

The diffusivity of Si in the $\alpha$-phase is relatively high. The composition of the first solid to form is the same as for the G-S model, but diffusion of Si through the cored dendrite arm brings all the $\alpha$-phase above the solubility limit. At the end of solidification the Si content of the $\alpha$-phase is essentially uniform at the composition given by the tie triangle for the eutectic temperature.

The diffusion of $\mathrm{Cu}$ is two to three times slower than for Si. The dendrites are still cored with respect to $\mathrm{Cu}$. The $\mathrm{Cu}$ content at the center of the dendrite arm has increased from $0.49 \%$ to $0.66 \%$. About $65 \%$ of the $\mathrm{Cu}$ in the overall alloy is within the $\theta$-phase in the ternary eutectic mixture in the interdendritic region. The $\theta$-phase accounts for $56 \%$ of the ternary eutectic and less than $4.3 \%$ of the overall alloy.

2.3.3.3 Comparison of Nucleation from the Liquid and from a Solid Matrix According to the assumption of local equilibrium, $\alpha$-phase is assumed to nucleate and grow from the liquid as soon as the phase becomes thermodynamically stable. There is negligible barrier to nucleation. The same assumption applies to nucleation and growth of secondary constituents, in this alloy silicon-phase and $\theta$-phase $\left(\mathrm{CuAl}_{2}\right)$, from the liquid. When the average concentration of a finite element enters a two-phase or three-phase region, each of the additional equilibrium phases is assumed to form, instantaneously. The composition of the phase and the relative amount of the phase within the local finite element adjust immediately to be consistent with the tie line or tie triangle for the average composition of that finite element (of thickness $\Delta \lambda$ ). (The only exception made herein with regard to nucleation from the liquid is for the divorced eutectic (DE-1) model, to be presented below. In that case the nucleation of silicon from either the $\alpha$-matrix or the liquid is delayed until a predetermined amount of undercooling.) 


\section{Section 2: Solutionizing Prediction}

In binary eutectic alloys, including $\mathrm{Al}-\mathrm{Si}$ and $\mathrm{Al}-\mathrm{Cu}$, solute solubility in the primary phase decreases sharply with decreasing temperature below the eutectic temperature. Thus, regions that solidify as a single phase may form fine precipitates while cooling after solidification. If silicon particles do not nucleate within the primary $\alpha$-phase dendrites of Al-Si alloys, the Si can diffuse from the dendrite cores to the interdendritic microconstituent, where it can be assumed, the $\alpha$-phase can quickly adjust to the reduced Si solubility. This will give rise to the so-called "anomalous microsegregation" where Si is observed to decrease from the center of the dendrites to the interdendritic regions.

In ternary eutectic systems, including $\mathrm{Al}-\mathrm{Si}-\mathrm{Cu}$, solubility in the primary phase may decrease during solidification (below the binary eutectic temperature and above the ternary eutectic temperature). Fine precipitates may form in the dendrite cores as the alloy solidifies as well as during post-solidification cooling.

The routines of CASTSEG and CASTSOLN are capable of accepting any amount of undercooling or supersaturation prior to nucleation of solid phases from either the melt or from another solid phase. As predictions of the models have been compared to measured solute distributions, it has been observed that the assumptions of negligible barrier to nucleation from the liquid and an infinite barrier to nucleation from the solid form suitable models for the solidification and post-solidification cooling of Al-Si-Cu alloys. Anomalous microsegregation is observed for Si-distributions in multicomponent alloys and, to a lesser extent, for Cu-distributions.

\subsubsection{Comparison of Nucleation from the Liquid and from a Solid Matrix As the alloy} cools below the ternary eutectic temperature, no solute redistribution occurs according to the assumption of the Gulliver Scheil model, negligible diffusion in solid phases. The composition of each finite element is unchanged as the as-solidified microstructure cools, at any rate, to room temperature. Solute redistribution in real alloys and in the LSD model is very dependent on the ratio of the cooling rate below the eutectic temperature to the cooling rate through the freezing 


\section{Section 2: Solutionizing Prediction}

range. Slow solidification rates, long solidification times, produce large dendrite spacing, and rapid cooling below the eutectic temperature will have little influence on solute redistribution. Conversely, rapid solidification rates, short solidification times, produce fine dendrites, and slow cooling below the eutectic temperature will have a major influence on solute redistribution.

Figure 2.3-7 compares the $\mathrm{Cu}$ and $\mathrm{Si}$ distributions for an $\mathrm{Al}-6.5 \% \mathrm{Si}-3.5 \% \mathrm{Cu}$ alloy (LSD model) that has been solidified adjacent to a copper chill for two extreme assumptions for nucleation of silicon-phase and $\theta$-phase particles from supersaturated $\alpha$-phase. At one extreme, it is assumed there is no undercooling required to precipitate silicon or $\mathrm{CuAl}_{2}$ particles as the solubility of $\mathrm{Si}$ and $\mathrm{Cu}$ decrease in the $\alpha$-phase as temperature decreases. The average composition at each position does not change from the average $\mathrm{Si}$ and $\mathrm{Cu}$ concentrations existing at the eutectic temperature. The $\mathrm{Si}$ and $\mathrm{Cu}$ concentrations in the $\alpha$-phase decrease to the solubility limits at the temperature where diffusion is assumed to become negligible, $300^{\circ} \mathrm{C}$. At the other extreme, it is assumed the barrier to nucleation is infinite. Consequentially, as the temperature decreases below the ternary eutectic temperature and the solubility of $\mathrm{Cu}$ and $\mathrm{Si}$ in the $\alpha$-phase decreases, $\mathrm{Cu}$ and $\mathrm{Si}$ diffuse away from the dendrite cores to the interdendritic regions. In the interdendritic regions the $\mathrm{Si}$ and $\mathrm{Cu}$ in the $\alpha$-phase can be easily transferred to the silicon-phase and $\theta$-phase particles that formed from the liquid. In the single $\alpha$-phase region, both the average alloy composition and the $\mathrm{Si}$ and $\mathrm{Cu}$ concentrations decrease. Because the diffusivity of $\mathrm{Si}$ is more than double the diffusivity of $\mathrm{Cu}$, the $\mathrm{Si}$ distribution approaches more closely to equilibrium. The later assumption is closer to the results of measurements.

2.3.3.5 Enhanced Solid Diffusion Model-1 We have measured, at temperatures between 720 $\mathrm{K}$ and $790 \mathrm{~K}$, the diffusivity of copper in a two-phase $\alpha+$ silicon region is up to five times the copper diffusivity in the single-phase $\alpha$-region (section 3.5). As a modification to the LSD model, the enhanced solid diffusion model-1 (ESD-1) uses a higher diffusivity for copper in the $\alpha$-phase of the two-phase region. (Model ESD-2 based on enhanced diffusivity of $\mathrm{Cu}$ and $\mathrm{Si}$ in the presence of $\mathrm{Mg}$ is discussed in section 2.3.4.) The two-phase, binary eutectic, interdendritic 


\section{Section 2: Solutionizing Prediction}

region will be a short circuit path for $\mathrm{Cu}$ diffusion. Accordingly, diffusion of copper through the dendrite core is expected to be the rate-limiting step when solutionizing microsegregation in cast Al-Si-Cu alloys.

The effect on the $\mathrm{Cu}$ distribution in the $\alpha$-phase of a five-fold increase in the diffusivity of copper in the $\alpha+$ silicon region (ESD-1 model) is presented in Figure 2.3-8. Diffusion of $\mathrm{Cu}$ through the dendrite core appears to limit solute redistribution. Slightly higher $\mathrm{Cu}$ content is predicted for the $\alpha$-phase within the $\alpha+$ silicon region. As a result the percentage nonequilibrium $\theta$-phase at the end of solidification decreases about $1 / 4 \%$, from $4.25 \%$ for LSD to $4.0 \%$ for ESD-1.

2.3.3.6 Divorced Eutectic Models Formation of a divorced eutectic in binary and multicomponent Al-Si alloys is consistent with numerous thermal and microstructural observations. Compare, for example, the microstructure of Figure 2.3-1, and the LSD model predictions in Figure 2.3-7. The primary $\alpha$-phase appears to occupy about $80 \%$ of the microstructure (Figure 2.3-1) and the silicon constituent appears to occupy about 50\% of the interdendritic regions. The LSD model predicts that the binary eutectic will begin to form after only about $40 \%$ of single $\alpha$-phase solidification and the silicon-phase will account for only $10 \%$ of the binary eutectic constituent. Particle pushing (silicon particles pushed to interdendritic regions by the advancing $\alpha / \mathrm{L}$ interface.) is one possible explanation. Eutectic divorcement is a second explanation, the one that is supported by interrupted solidification experiments, and the mechanism for microstructure evolution that was included in solidification models. Two divorced eutectic models were explored.

In the first divorced eutectic model (DE-1) the assumptions for solidification and solute redistribution within a characteristic dendrite volume element are the same as for the LSD model except for the assumption of local equilibrium, which is modified, as follows. 


\section{Section 2: Solutionizing Prediction}

- The solute enriched interdendritic liquid undercools before nucleation of the silicon-phase. The silicon phase does not nucleate as soon as the liquid becomes saturated with respect to Si. Equilibrium is assumed at all interfaces once the silicon phase nucleates.

Undercooling prior to nucleation of the silicon-phase extends the primary phase region and is expected to increase the fraction of silicon-phase and the average silicon concentration within the two-phase $\alpha+$ silicon interdendritic region.

To explore the effect of delayed nucleation of silicon-phase (DE-1 model) solidification was simulated with the assumption the silicon-phase nucleates at $813 \mathrm{~K}$. All other assumptions are the same as for the LSD model. For the LSD model, the liquid composition reaches the line of two-fold saturation at $841 \mathrm{~K}$ and silicon-phase nucleates immediately. In the DE-1 model $\alpha$-phase continues to grow between $841 \mathrm{~K}$ and $813 \mathrm{~K}$. When the silicon-phase nucleates at $841 \mathrm{~K}$ (28 K undercooling) the composition of the $\alpha$-phase is $0.89 \% \mathrm{Cu}$ and $1.55 \% \mathrm{Si}$. The fraction silicon-phase in the $\alpha+$ silicon region is $15 \%$ for the DE- 1 model and $10 \%$ for the LSD model. The fraction of nonequilibrium $\theta$-phase at the end of solidification is $1 / 2 \%$ less than for the LSD model. The distributions of $\mathrm{Cu}$ in the $\alpha$-phase are compared for the DE-1 and LSD models in Figure 2.3-9.

The predictions of the DE-1 model are more consistent with the microstructure in Figure 2.3-1 than the predictions of the previous models. The fraction of primary phase dendrites $(\alpha$ and $\alpha+$ silicon' $^{\prime}$ regions) is $43 \%$ for the other three models and $59 \%$ for the DE- 1 model. The predictions are moving in the right direction. It would be tempting to assume larger undercooling prior to nucleation of the silicon-phase in the binary eutectic. But, such a large undercooling would not be consistent with thermal measurements. Instead, a second mechanism of eutectic divorcement is proposed. 


\section{Section 2: Solutionizing Prediction}

In the second divorced eutectic model (DE-2, called middle growth by the developer) the assumptions for solidification and solute redistribution within a characteristic dendrite volume element are the same as for the LSD model. The geometry and interface advance rate are modified.

- Growth of the binary eutectic proceeds at multiple interfaces with the enriched interdendritic liquid. When the eutectic valley is reached, $\alpha$-phase continues to solidify at the existing $\alpha / 1$ interface $\left(\lambda^{*}\right)$ with the liquid and simultaneously a binary eutectic colony forms and grows in the interdendritic liquid pool. The eutectic colony has two interfaces with the liquid where $\alpha$-phase, silicon-phase, and liquid are in equilibrium. The solute enriched interdendritic liquid does not undercool before nucleation of the silicon-phase. The silicon phase does nucleate as soon as the liquid becomes saturated with respect to Si. Equilibrium is assumed at all interfaces at all stages of solidification.

- The overall solidification rate is unchanged (equation 2.3-2) as the binary eutectic mixture solidifies. Keeping in mind that the temperature continues to decrease, along the eutectic valley, as the binary eutectic forms, the value for $f_{\mathrm{E}}$ substituted into equation 2.3-2 is the fraction of isothermally forming ternary eutectic microconstituent.

The divorced eutectic solidification model is represented, schematically, in Figure 2.3-10. The divorced eutectic solidification model is applied to the solidification and post-solidification cooling of an $\mathrm{Al}-7 \% \mathrm{Si}-3.5 \% \mathrm{Cu}$ alloy in Figure 2.3-11 and compared to the predictions of the simple LSD model. As designed, the divorced eutectic model predicts an appropriate fraction of primary phase, an appropriate ratio of silicon and $\alpha$-phases in the interdendritic binary eutectic colony. The $\mathrm{Cu}$ distribution in the a-phase differs only slightly. The Si distribution in the $\alpha$-phase differs considerably. The average Si content also will differ considerably between models. The combination of the ESD and DE-2 models are in good agreement with detailed measurements of solute distribution and phase fraction in Al-Si-Cu ternary alloys (section 3.4).

\subsubsection{Application of CASTSEG to $\mathrm{Al}-7 \% \mathrm{Si}-3.5 \% \mathrm{Cu}-0.5 \% \mathrm{Mg}$}




\section{Section 2: Solutionizing Prediction}

The solidification sequence in quaternary $\mathrm{Al}-\mathrm{Si}-\mathrm{Cu}-\mathrm{Mg}$ casting alloys proceeds from primary $\alpha$-phase formation from the liquidus to the surface of two-fold saturation, to $\alpha+$ silicon (binary eutectic) solidification through the three phase region; to $\alpha+$ silicon + Q-phase, followed by the invariant quaternary eutectic of $\alpha+$ silicon + Q-phase $+\theta$. The LSD model can be applied to the ternary alloy using the procedures described in section 2.3.2.2 for ternary alloys adding one more degree of freedom to each multiphase region and adding one additional multiphase region to the solidification sequence. (See the discussion of phase relations in quaternary alloys, section 2.1.) The extensions to the LSD model described in section 2.3 .3 apply to the quaternary alloy plus one additional extension to account for the presence of $\mathrm{Mg}$ in the $\alpha$-matrix on the diffusion of $\mathrm{Cu}$.

2.3.4.1 Enhanced Diffusion Model (ESD-2) The presence of a small amount of $\mathrm{Mg}$ in the Al- $\alpha$-phase increases the diffusivity of $\mathrm{Cu}\left(\mathrm{D}_{\mathrm{CuCu}}\right)$ by a factor of two (section 3.5). This is the second enhanced diffusion effect. Copper diffusion is enhanced through the two-phase region due to the presence of silicon particles (ESD-1). As seen in Figure 2.3-9, short circuit diffusion through the two-phase region does not have a strong influence on reducing copper microsegregation. Diffusion of copper through the primary, single $\alpha$-phase region limits solute redistribution in the cored dendrite. The effect of $\mathrm{Mg}$ is to increase $\mathrm{Cu}$ diffusion in the primary phase region (ESD-2) and does have a significant effect on the resultant microsegregation in ascast $\mathrm{Al}-\mathrm{Si}-\mathrm{Cu}-\mathrm{Mg}$ alloys.

Figure 2.3-12 shows the prediction of CASTSEG for $\mathrm{Cu}$ distribution in the quaternary $\mathrm{Al}-7 \% \mathrm{Si}-3.5 \% \mathrm{Cu}-0.5 \% \mathrm{Mg}$ alloy at the end of solidification (just below the quaternary eutectic temperature) according to the LSD model and the enhanced diffusion model (ESD-2). The Cu content predicted for the center of the dendrite arm $\left(\mathrm{C}_{\min }\right)$ is approximately $1 \% \mathrm{Cu}$, in good agreement with measurements.

2.3.4.2 Comparison with Measurement Figure 2.3-13 compares to measurements the predictions of the various models for $\mathrm{Cu}$ distribution after solidification and post-solidification 


\section{Section 2: Solutionizing Prediction}

cooling to $300^{\circ} \mathrm{C}$ for an $\mathrm{Al}-7 \% \mathrm{Si}-3.5 \% \mathrm{Cu}-0.5 \% \mathrm{Mg}$ alloy. The combination of the LSD model with the divorced eutectic model DE-2 and with both enhanced diffusion models ESD-1 and ESD-2 predicts a final, as-cast $\mathrm{Cu}$ distribution in very good agreement with the result of electron microprobe measurements.

\section{$\underline{\text { 2.3.5 Two-Dimensional Plate-like Characteristic Volume Element }}$}

Figure 2.3-10 (lower) illustrates a 2-D plate-like volume element that has been implemented by CASTSEG. The overall predictions of the 2-D and 1-D plate-like models for solute distribution after solidification and post-solidification cooling are comparable. The geometric distribution of elements and phases predicted by the 2-D model provides better input for CASTSOLN for combined simulation of solidification and solution treatment. The 2-D model will be discussed further in section 2.4. CASTSOLN.

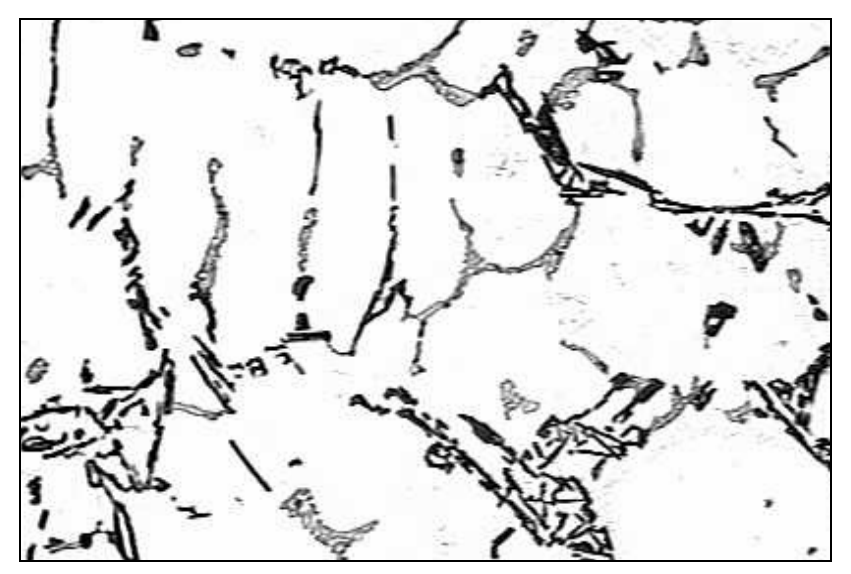

Figure 2.3-1. Al-6.5\% Si-3.5\% Cu, cast near a chill. White matrix is $\alpha$, dark gray particles are silicon, and light gray particles are $\theta$-phase. (Unetched, $350 \mathrm{X}$.) 


\section{Section 2: Solutionizing Prediction}

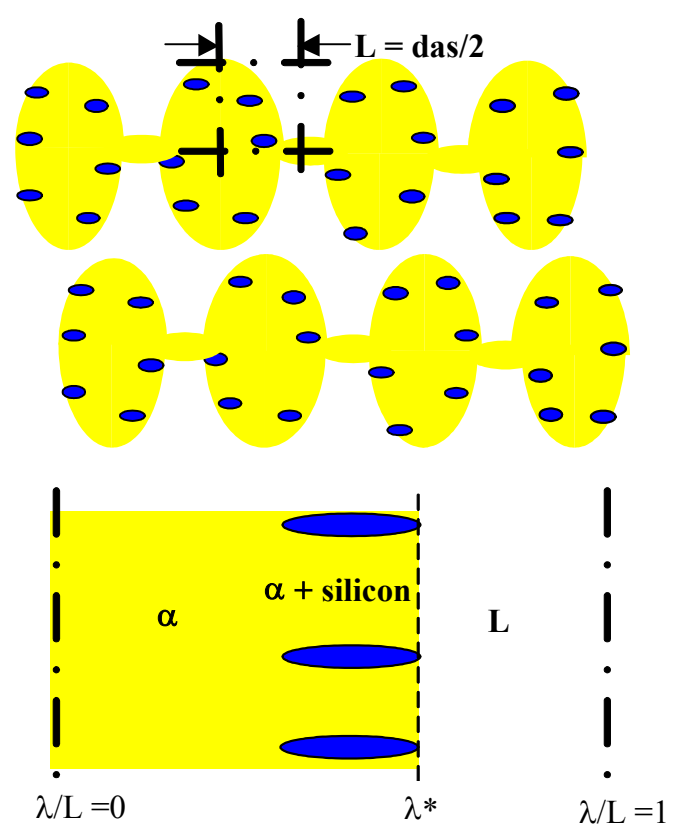

Figure 2.3-2. Top: An array of dendrites of Al-Si-Cu alloy showing the position of the 1-D volume element. Bottom: The volume element showing a dendrite core of $\alpha$-phase and $\alpha$-phase + silicon-phase growing into the interdendritic liquid

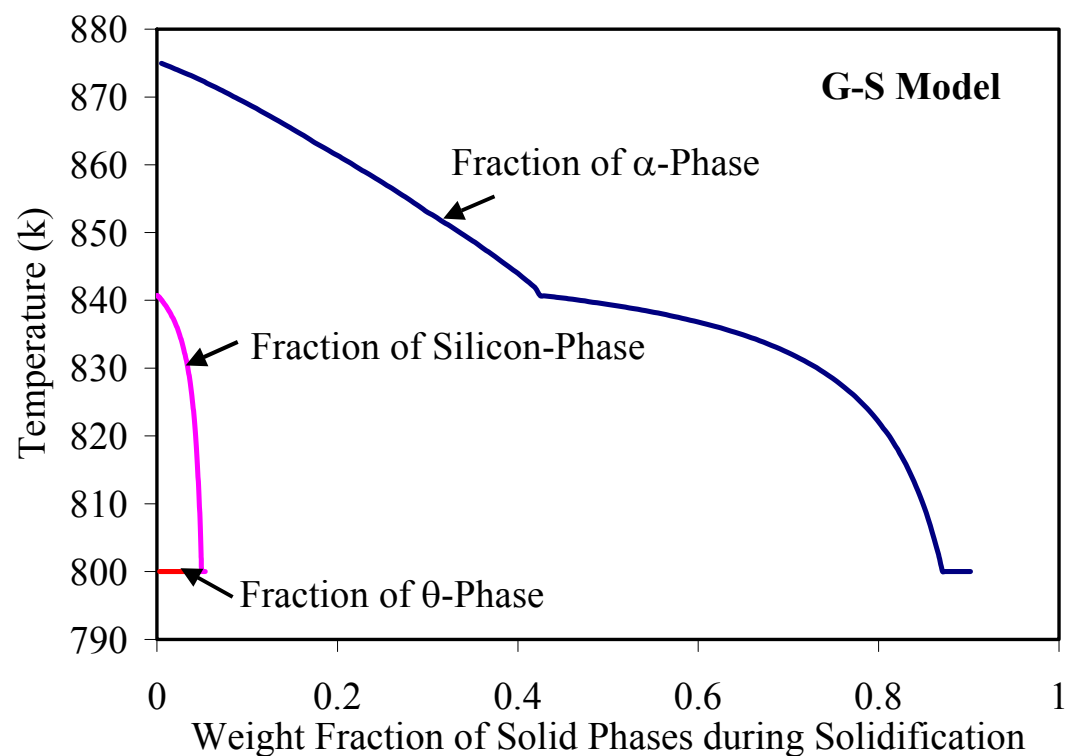

Figure 2.3-3. Solidification curve for Al-6.5\% $\mathrm{Si}-3.5 \% \mathrm{Cu}$ for the Gulliver-Scheil (G-S) model 


\section{Section 2: Solutionizing Prediction}

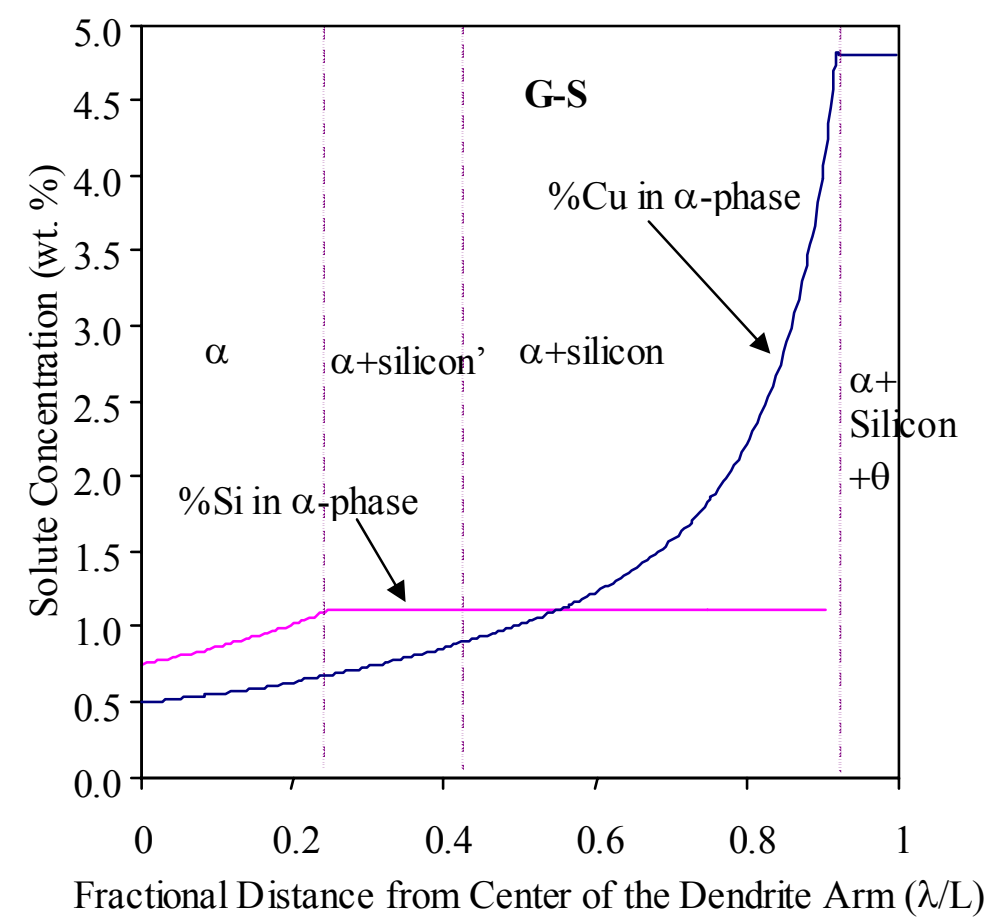

Figure 2.3-4. Distribution of $\mathrm{Si}$ and $\mathrm{Cu}$ in the $\alpha$-phase across the dendrite volume element Gulliver Scheil model

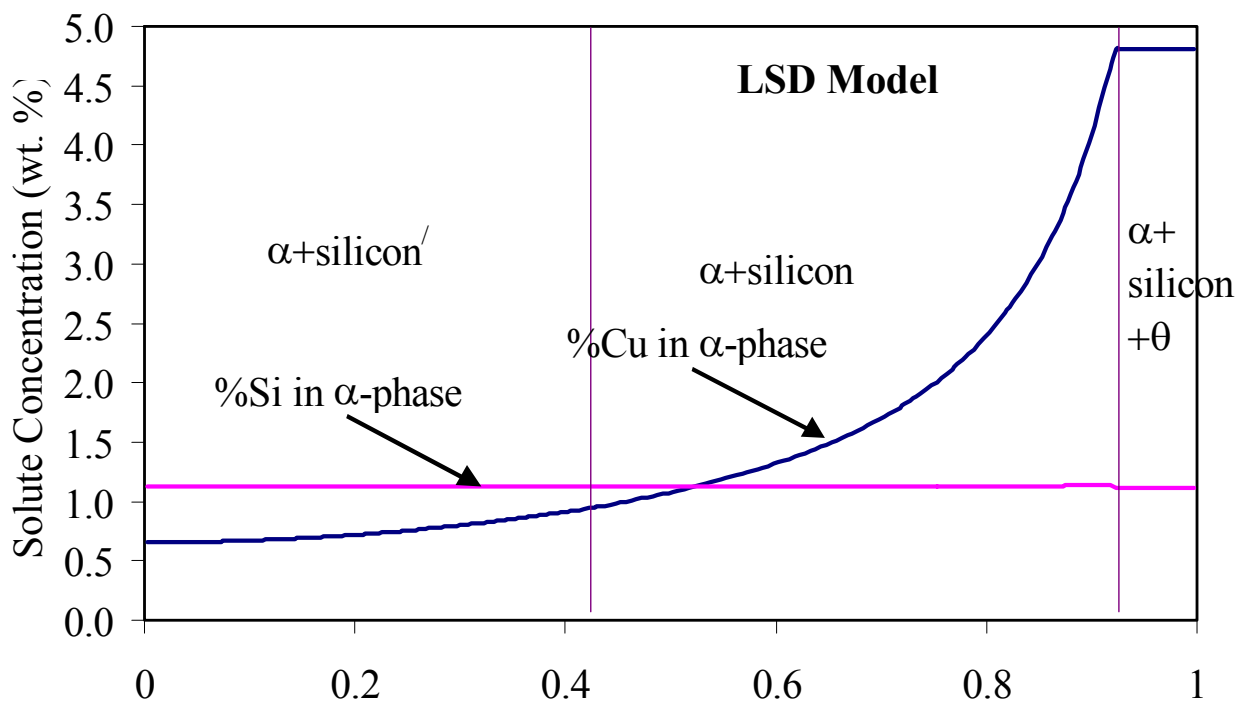

Fractional Distance from Center of the Dendrite $\operatorname{Arm}(\lambda / \mathrm{L})$

Figure 2.3-5. Distribution of $\mathrm{Si}$ and $\mathrm{Cu}$ in the $\alpha$-phase across the dendrite volume element limited solid diffusion model 


\section{Section 2: Solutionizing Prediction}

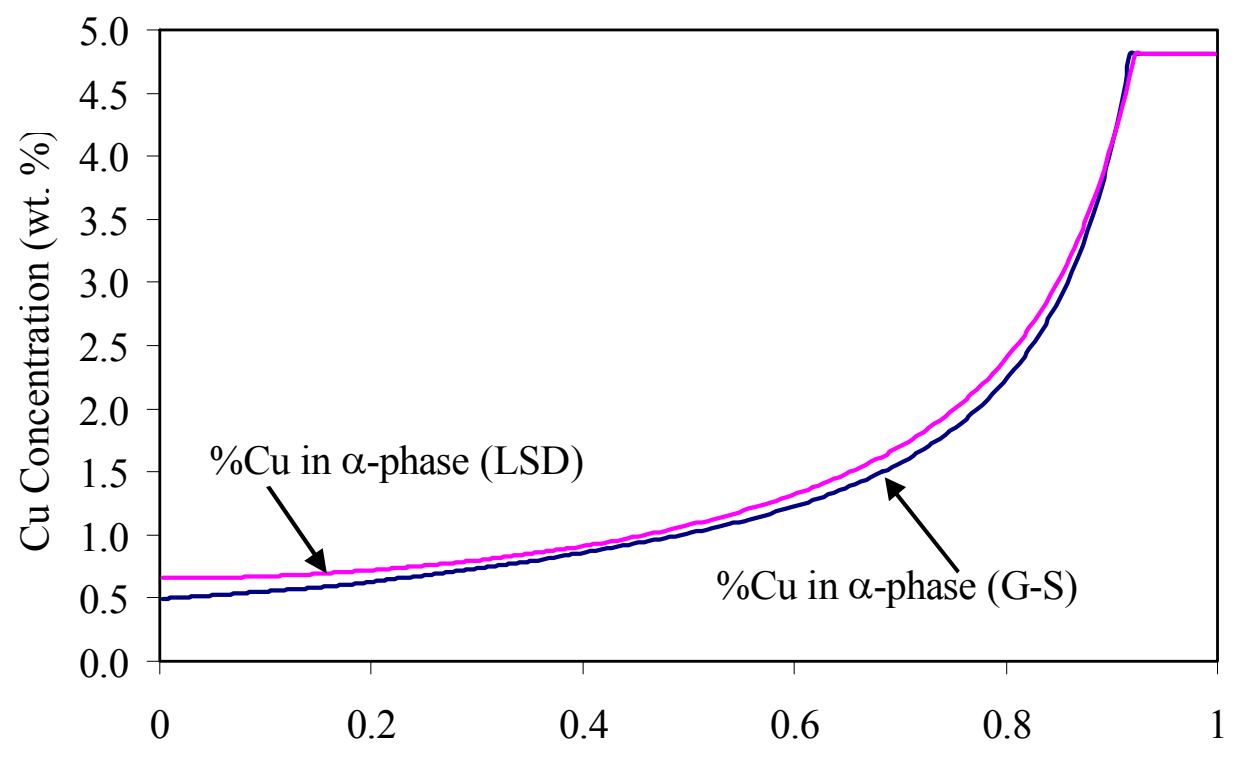

Fractional Distance from Center of the Dendrite Arm $(\lambda / \mathrm{L})$

Figure 2.3-6. Comparison of $\mathrm{Cu}$ distribution in the $\alpha$-phase for the Gulliver-Scheil (G-S) and limited solid diffusion (LSD) models 


\section{Section 2: Solutionizing Prediction}

Distribution of Cu across a Dendrite Arm (Position 1, 580K)

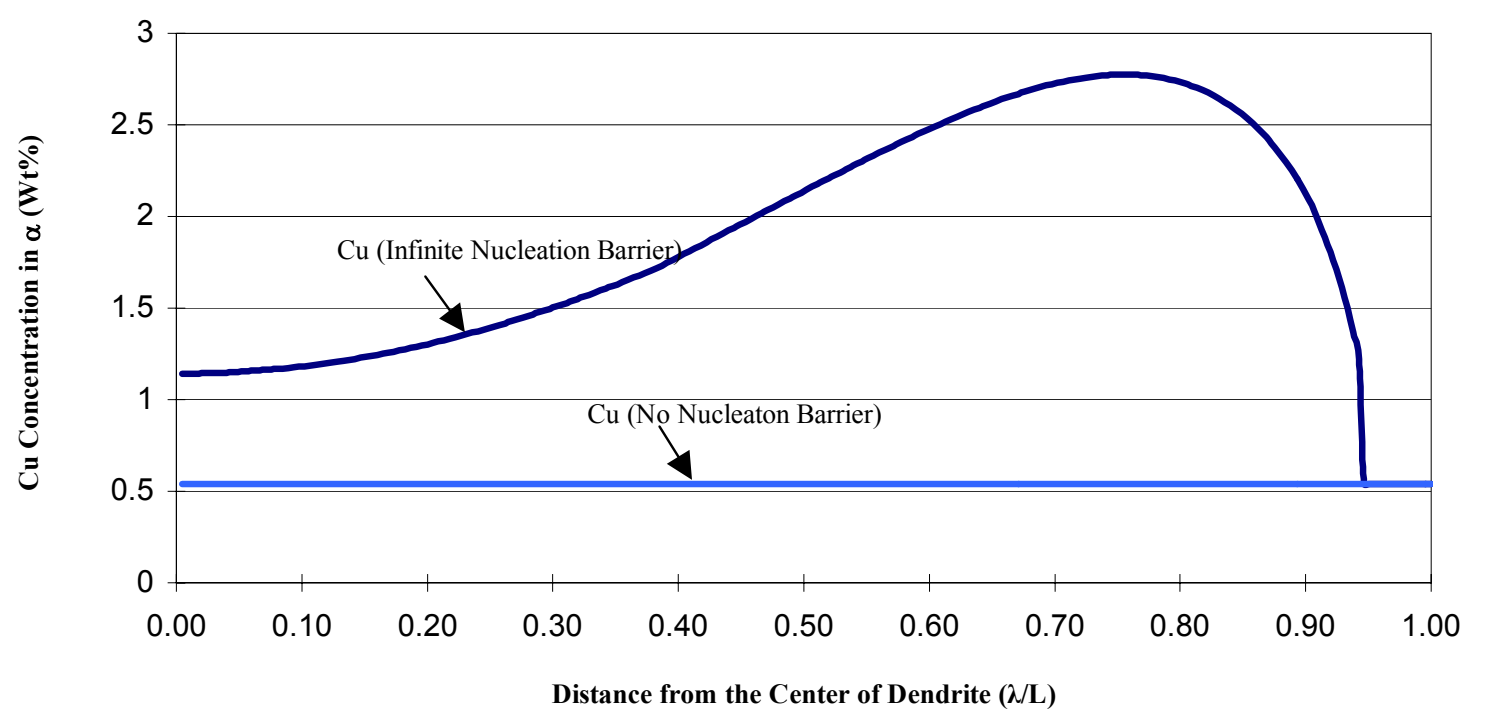

Si Distribution across a Dendrite Arm of Al-7Si-3.5Cu (Position 1, 580K)

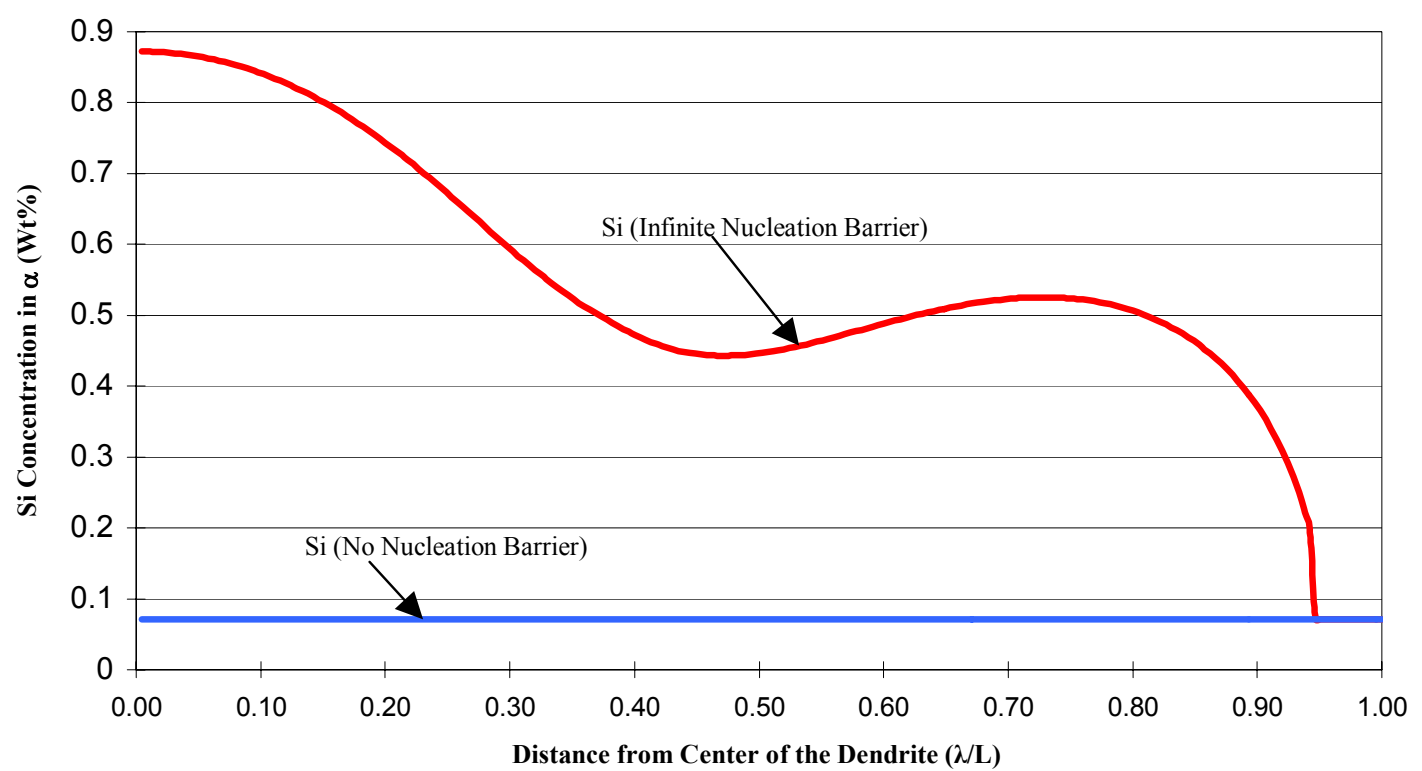

Figure 2.3-7. Comparison of $\mathrm{Cu}$-distribution (top) and Si-distribution (bottom) in the $\alpha$-phase after cooling below the ternary eutectic to $573 \mathrm{~K}$, assuming no nucleation of silicon or $\theta$-phase particles from the $\alpha$-matrix (infinite nucleation barrier) vs. no undercooling before nucleation of precipitates from the $\alpha$-matrix (no nucleation barrier) 


\section{Section 2: Solutionizing Prediction}

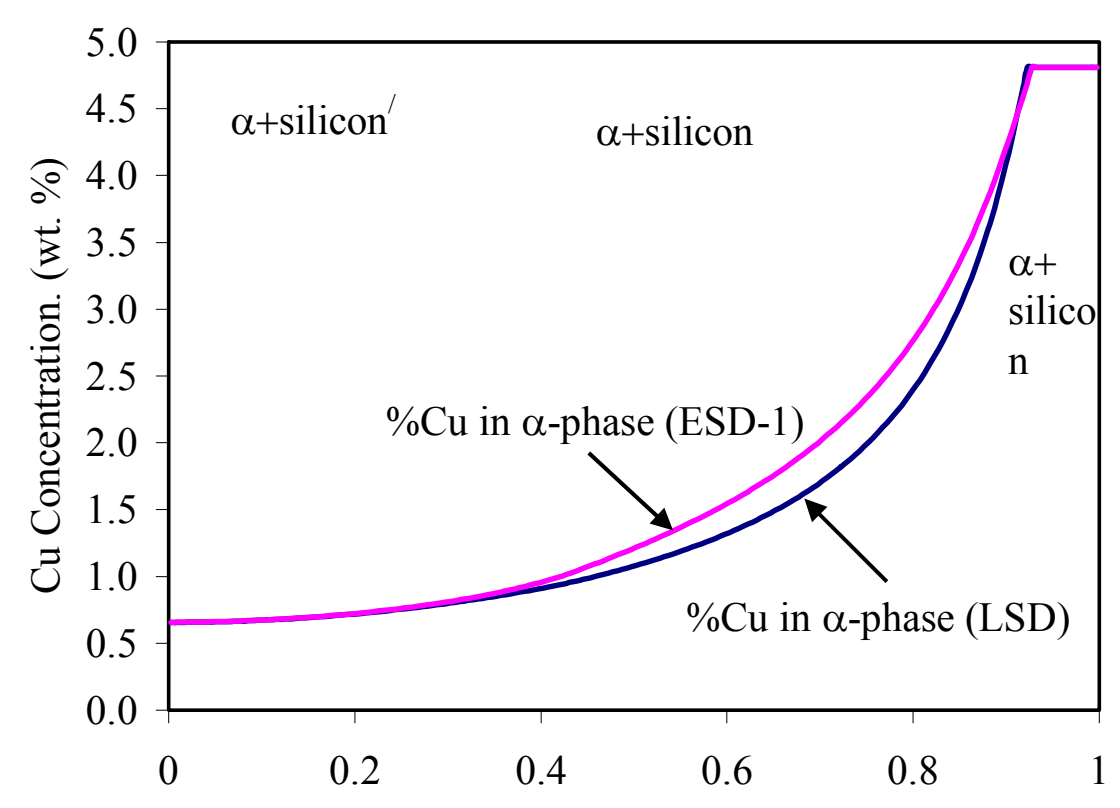

Fractional Distance from Center of the Dendrite Arm $(\lambda / \mathrm{L})$

Figure 2.3-8. Comparison of $\mathrm{Cu}$ distribution in the $\alpha$-phase for the enhanced diffusion and limited solid diffusion models

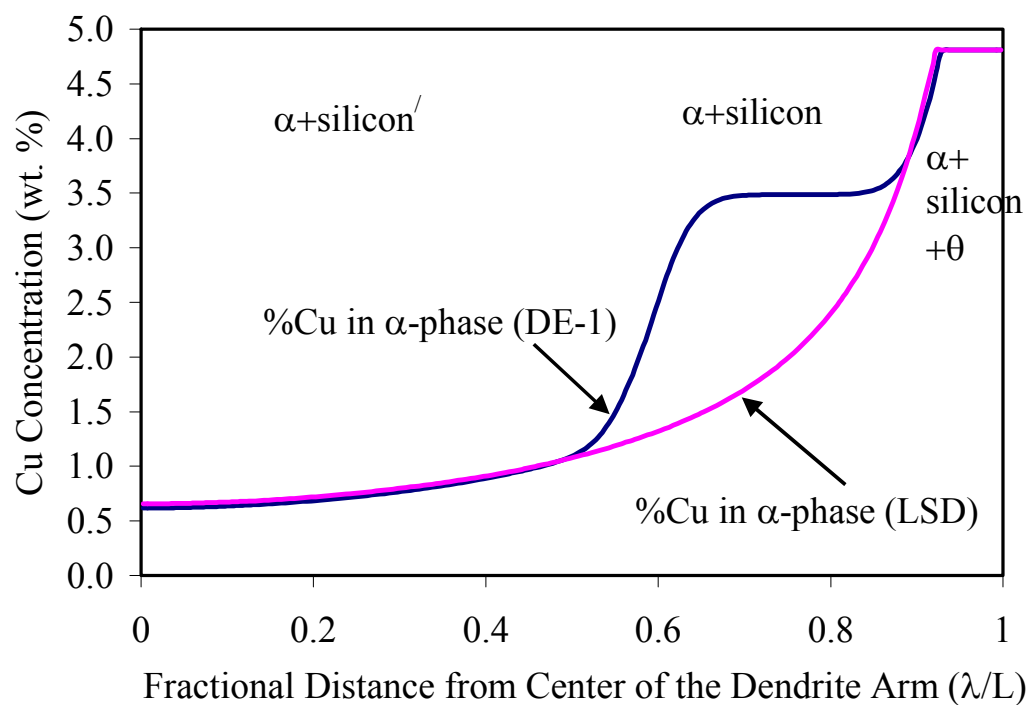

Figure 2.3-9. Comparison of $\mathrm{Cu}$ distribution in the $\alpha$-phase for the limited solid diffusion and divorced eutectic models 


\section{Section 2: Solutionizing Prediction}

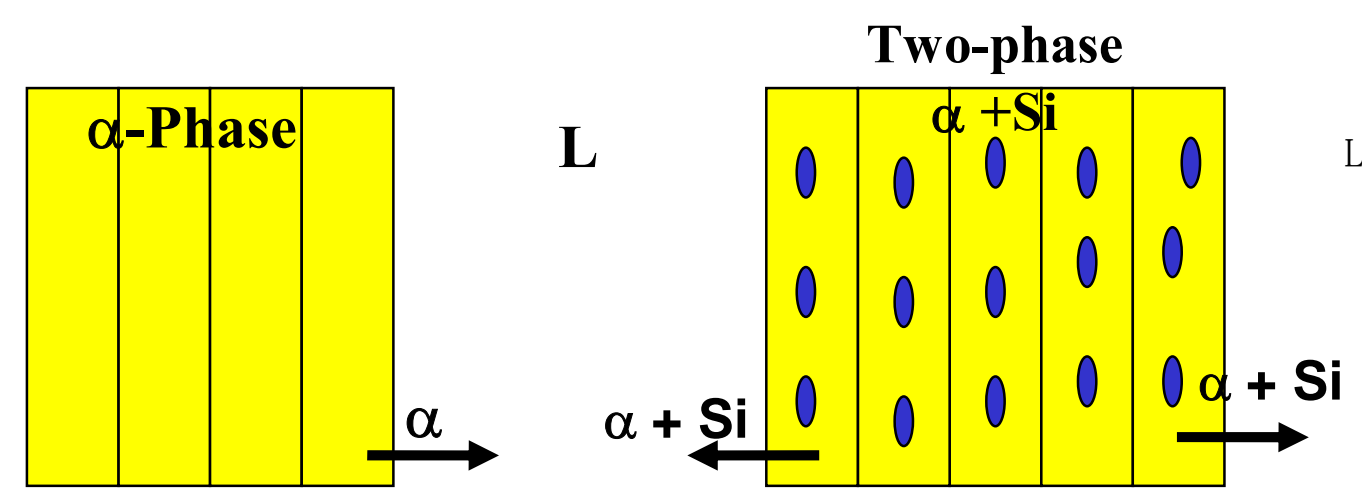

Divorced Eutectic Model-2

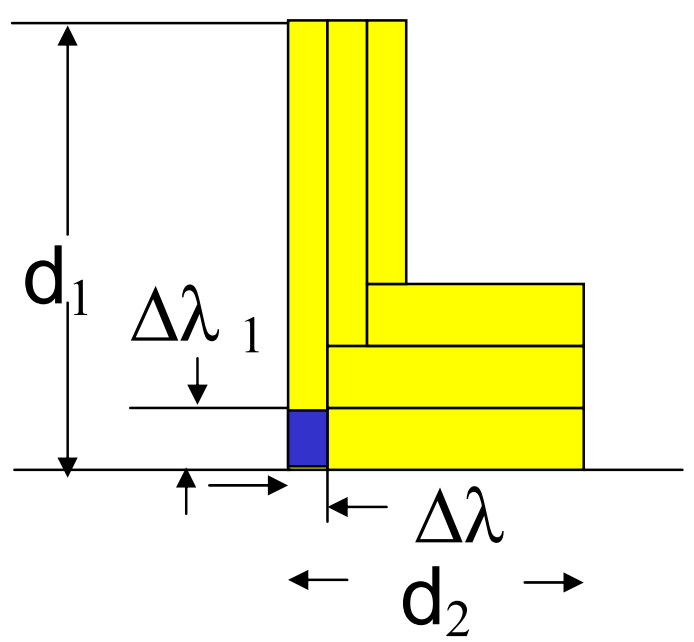

2-D Plate-like Volume Element

Figure 2.3-10. Extended models for solidification and solution treatment simulation. Upper: Model (DE-2) for divorced eutectic in ternary and higher order alloys. Growth of eutectic mixture is spread over three interfaces. Primary $\alpha$-phase continues to grow in equilibrium with enriched liquid at edge of original dendrite arm. Mixture of $\alpha+$ silicon grows in eutectic colony in equilibrium with same enriched liquid. Lower: Two-dimensional characteristic volume element, schematic. The number of divisions in the 1-direction is the same as the number of divisions in the 2-direction. The ratio of dimensions to the differential volume element $\left(\Delta \lambda_{2} / \Delta \lambda_{1}\right)$ are in the same proportion as the characteristic spacings $\left(d_{2} / d_{1}\right)$. 


\section{Section 2: Solutionizing Prediction}

Cu Distribution in Alpha-Phase (Al-7\%Si-3.5\%Cu, DAS=38 Microns, 573K)

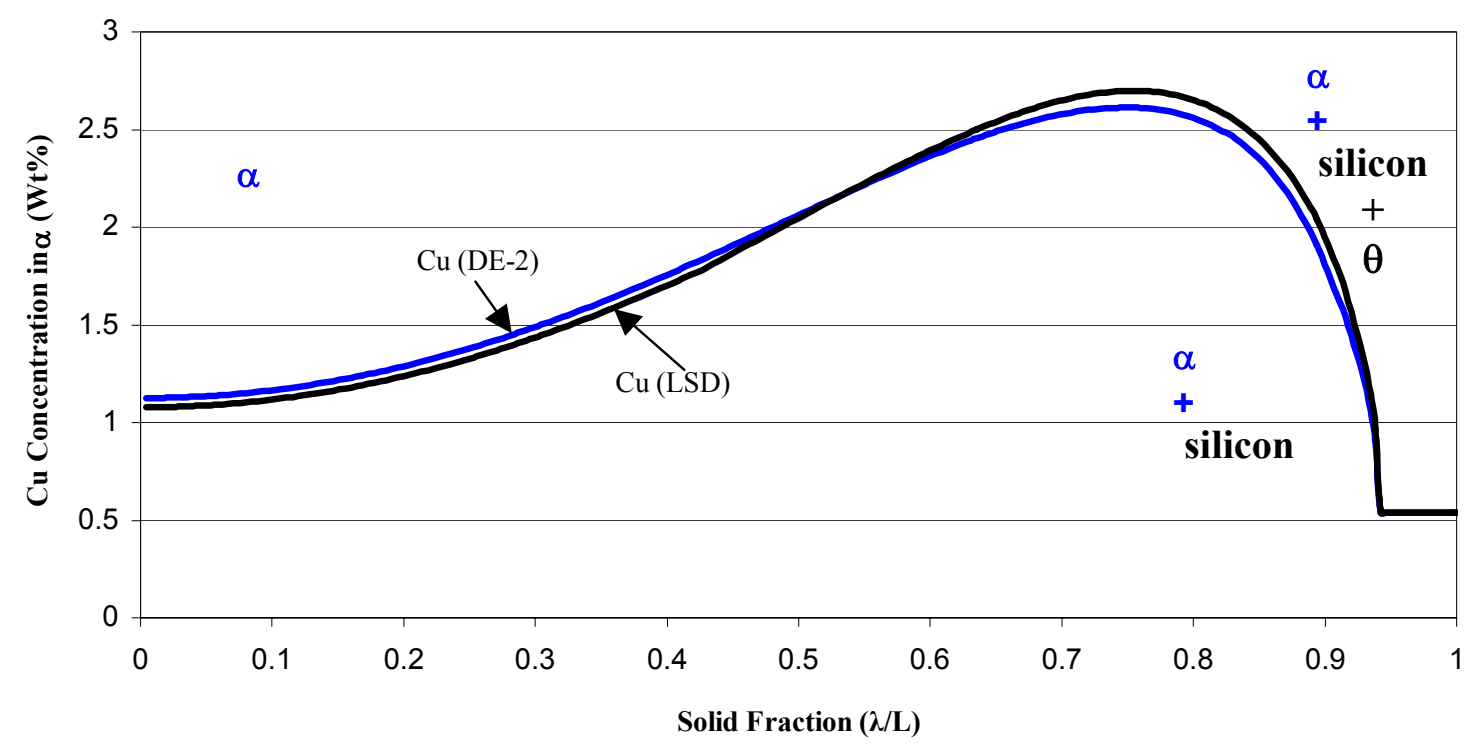

Si in Alpha-Phase (Al-7\%Si-3.5\%Cu, DAS=38 Microns, $573 \mathrm{~K})$

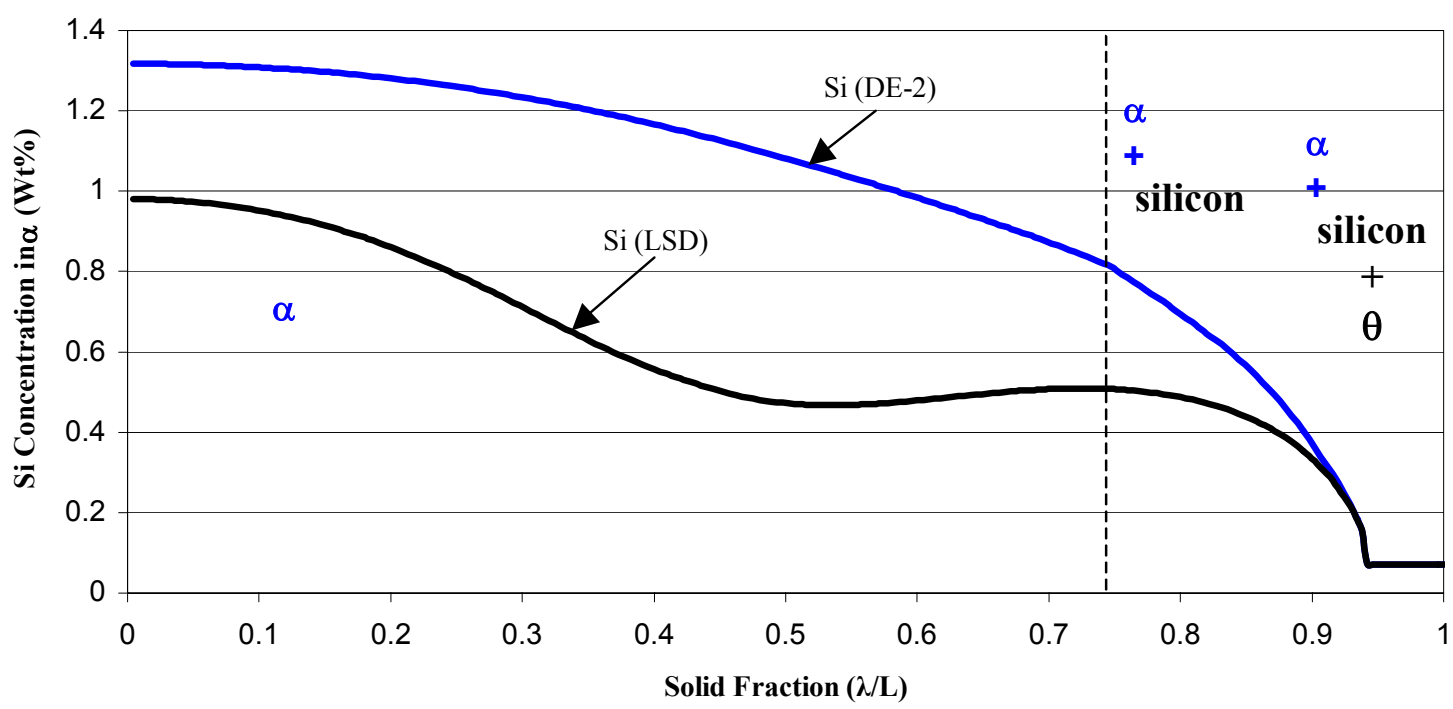

Figure 2.3-11. Comparison of the $\mathrm{Cu}$ (upper) and $\mathrm{Si}$ (lower) distribution after solidification and cooling to $300^{\circ} \mathrm{C}$ for an $\mathrm{Al}-7 \% \mathrm{Si}-3.5 \% \mathrm{Cu}$ alloy predicted by the original LSD model and the divorced eutectic model. Dashed line indicates extent of primary $\alpha$-phase for DE-2 model. 


\section{Section 2: Solutionizing Prediction}

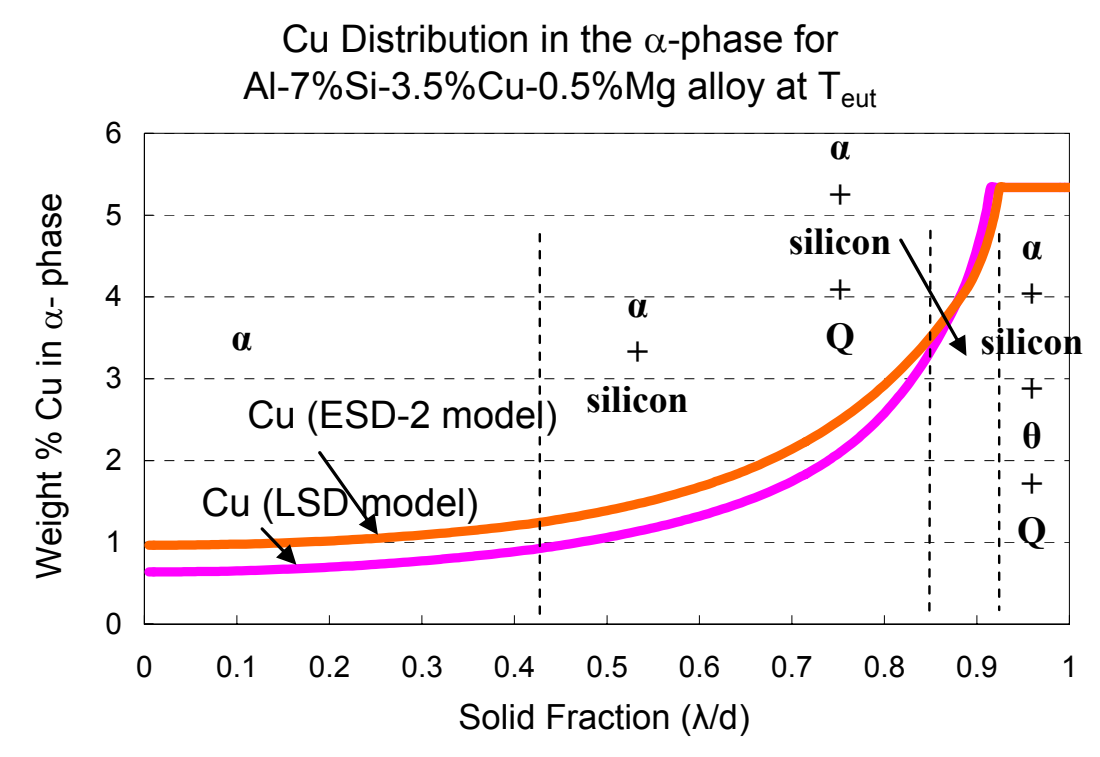

Figure 2.3-12. $\mathrm{Cu}$ distribution in $\alpha$-phase for $\mathrm{Al}-7 \% \mathrm{Si}-3,5 \% \mathrm{Cu}-0.5 \% \mathrm{Mg}$ alloy after solidification to the quaternary eutectic temperature: Comparison of use of $\mathrm{D}_{\mathrm{CuCu}}$ (LSD) measured for ternary alloy without $\mathrm{Mg}$ and use of enhanced diffusivity $\left(2 \mathrm{xD}_{\mathrm{CuCu}}\right)$ for $\mathrm{Mg}$ addition (ESD-2)

Cu Distribution in $\alpha$-phase (Al-7\%Si-3.5\%Cu-0.5\%Mg, DAS=38 $\mu \mathrm{m}, \mathrm{T}=573 \mathrm{~K}$ )

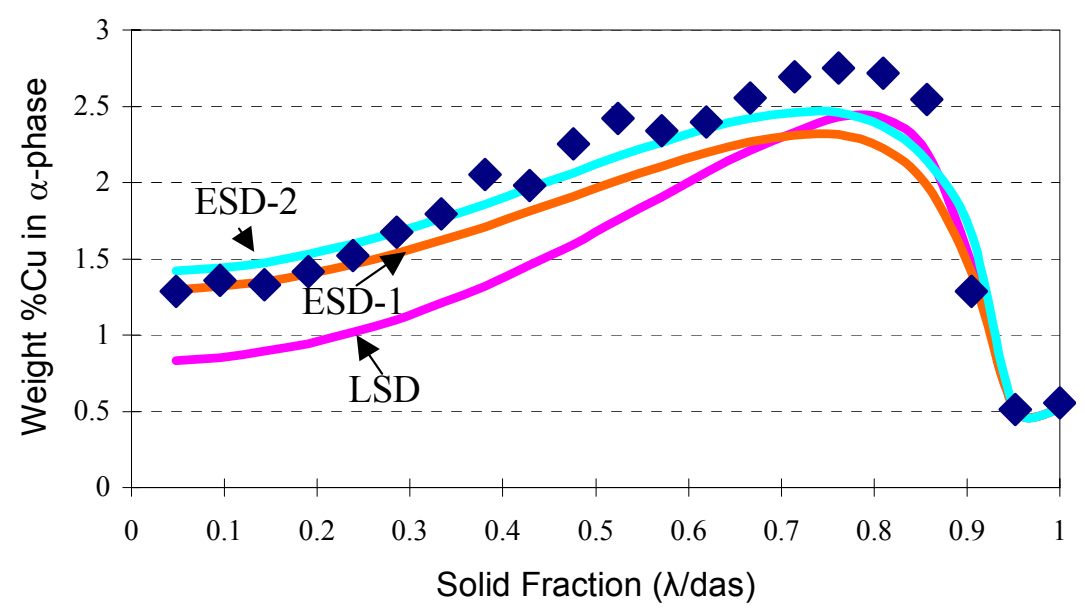

Figure 2.3-13. Comparison of predicted $\mathrm{Cu}$ distribution in $\alpha$-phase for as-cast Al-7\% $\%$ i-3.5\%Cu-0.5\%Mg with microprobe data (points). Model ESD-2 includes divorced solidification, enhanced diffusivity due to $\mathrm{Mg}$ addition and enhanced diffusion in $\alpha+$ silicon region 


\section{Section 2: Solutionizing Prediction}

\subsection{CASTSOLN}

The major objective of the module CASTSOLN is to quantitatively describe the evolution of as-cast microstructure (phase amounts, morphologies, and solute distribution) during post-solidification thermal cycles including, in particular, solution heat treatment of industrial multi-component alloys. The initial microstructural data for this module may be input from the module CASTSEG, from measurements, and from other simulation software. The module has been implemented for binary eutectic, ternary eutectic, and quaternary alloy systems. CASTSOLN has been used iteratively with experiment to validate and improve the underlying models and to gather kinetic and thermodynamic data. The basic model and symbols used for simulation of post-solidification thermal cycles are parallel to the treatment of solute redistribution during solidification, discussed for the CASTSEG module in the previous section (2.3).

Selection of the geometry and scale of the volume element to characterize the dendrite morphology and spacing is more critical to the simulation of phase dissolution and solute redistribution during solution treatment than for the simulation of phase formation and solute redistribution during solidification. A one-dimensional plate-like model based on the secondary arm spacing represents well the solute redistribution processes during solidification of multiphase and multicomponent aluminum casting alloys (sections 2.3 and 3.4). A two-dimensional plate-like volume element based on a combination of the secondary and primary arm spacing characterizes more closely the redistribution of solute during solution treatment. The dendrite arm spacing is determined by the amount of time liquid and solid are in contact during solidification, i.e., the local solidification time $\left(\theta_{\mathrm{f}}\right)$. Other features of the microstructures often scale in proportion to the dendrite arm spacing. Soluble intermetallic particles, which are of primary interest in simulation of solution heat treatment, usually are dispersed in the interdendritic regions. Constituent particle size scales in proportion to the secondary arm spacing and constituent particle spacing scales in proportion to secondary and primary arm spacing. For highly grain-refined aluminum alloys, the grain size approaches the 


\section{Section 2: Solutionizing Prediction}

secondary arm spacing and a 1-D sphere-like model based on a single-spacing effectively characterizes the as-cast microstructure.

\section{$\underline{\text { 2.4.1 Solution Treatment Models }}$}

2.4.1.1 Mass Transfer In the simulation of solution treatment, we assume the limiting factor for dissolution of soluble secondary phases is diffusion of solute through the primary phase dendrite. In the solidification simulation models as in reality, low melting, secondary phases form in the interdendritic regions ( $\lambda$ approaching $L$ and $f_{S}$ approaching 1 ). Local equilibrium pertains at the interfaces between the primary $\alpha$-phase and intermetallic particles, and the $\alpha$-phase in the multiphase microconstituents is at the solubility limit for the current temperature and local average composition. A general feature of as-cast microsegregation is that the primary phase dendrite is depleted in key solute elements. During solution treatment rate of the dissolution of soluble phases is controlled by diffusion of the solute from the multiphase regions into the dendrite cores that are, in general, depleted in solute. As discussed in the previous section, when an alloying element, such as $\mathrm{Si}$ in $\mathrm{Al}$, has a relatively high diffusivity in the $\alpha$-phase and its solubility decreases sharply with temperature, anomalous microsegregation is prevalent. During reheating and the initial stages of solution treatment, the effects of anomalous microsegregation will be reversed.

For a binary alloy, we assume the two-phase interdendritic eutectic constituent can adjust phase compositions and phase fractions within the interdendritic region to rapidly reach local equilibrium. In comparison, diffusion of solute down the gradient into the dendrite core requires longer range diffusion and, at least in the first stages of solution treatment, will be rate controlling. Within the two-phase region in a binary alloy there is no diffusion across the interdendritic region (in the $\lambda$-direction of the characteristic volume element). As solute diffuses to the dendrite core, the secondary phase particles dissolve at the boundary between the single and two phase regions. When the secondary phase dissolves totally, there will still be a 


\section{Section 2: Solutionizing Prediction}

concentration gradient within the primary phase dendrites. Continued solution treatment will tend to homogenize the dendrite.

For an n-component alloy, at the beginning of solution treatment, the single-phase dendrite core will be separated from the n-phase interdendritic eutectic microconstituent by two-phase to (n-1)phase regions in which both the compositions and fractions of the phases can vary at the solution treatment temperature. Again, it is diffusion across the characteristic volume element (in the $\lambda$ - direction) that is the limiting factor in dissolving the secondary phases. For a ternary alloy, as example, diffusion can occur across the primary phase within the single-phase dendrite cores and through the two-phase (binary eutectic) region. There is no net diffusion across the three-phase eutectic constituent. Dissolution of soluble secondary phases will occur at the interface between the two phase and three phase regions and throughout the two-phase region.

Carrying the illustration to quaternary eutectic alloy systems, using the Al-Si-Cu-Mg system in the vicinity of $\mathrm{Al}-7 \% \mathrm{Si}-3.5 \% \mathrm{Cu}-0.5 \% \mathrm{Mg}$ as an example, the single phase region will be aluminum-rich $\alpha$-phase, the two phase region will be $\alpha+$ silicon, the three phase region will be $\alpha+$ silicon + Q-phase, and the quaternary eutectic microconstituent will be $\alpha+$ silicon $+Q+\theta$. Assuming local equilibrium within the volume elements that have an average composition within the four-phase region, there will be no composition gradient within the $\alpha$-phase within the four-phase region and no potential for diffusion. Diffusion, solute redistribution, and phase dissolution can proceed within the two-phase and three-phase regions. Diffusion in the single-phase $\alpha$-region will proceed toward eliminating coring and approaching overall equilibrium.

2.4.1.2 Characteristic Volume Element The characteristic volume elements used by CASTSEG and CASTSOLN to simulate solute redistribution in dendritic microstructures is introduced in section 2.3 and in Figures 2.3-2 and 2.3-10. The 1-D plate-like model is illustrated for a ternary Al-Si-Cu alloy in Figure 2.3-2. The lower diagram in Figure 2.3-10 illustrates the 2-D plate-like model. The upper diagram in Figure 2.3-10 illustrates the divorced 


\section{Section 2: Solutionizing Prediction}

eutectic model (DE-2). The divorced eutectic is 1-D and plate-like and differs from the geometric model illustrated in Figure 2.3-2 only in the order of solidification of the individual volume elements of thickness $\Delta \lambda$ in CASTSEG. The result is a major shift in the as-cast solute distribution output by CASTSEG. Nonetheless, CASTSOLN treats both 1-D plate-like models the same way. The input of as-cast one-dimensional (in distance) solute distribution forms the initial state for the CASTSOLN solute redistribution computations.

\subsubsection{CASTSOLN Simulation software}

The procedures used by CASTSOLN are illustrated below using a binary and then a ternary alloy system as examples. Procedures applied to ternary alloy system are illustrative of the procedures applied to n-component (multicomponent) alloy systems.

\section{Input to the Solutionizing Routines}

1. Alloy \& Heat Treatment Process Parameters:

- Overall average (measured or calculated) solute concentrations of alloying elements in the initial melt. For a

$$
\begin{aligned}
& \text { binary alloy: } \mathrm{C}_{0} \\
& \text { ternary alloy: } \mathrm{C}_{10} \text { and } \mathrm{C}_{2} \\
& \text { quaternary alloy: } \mathrm{C}_{10}, \mathrm{C}_{20}, \mathrm{C}_{30} \\
& \text { n-component alloy: } \mathrm{C}_{10}, \mathrm{C}_{20}, \mathrm{C}_{30}, \ldots \mathrm{C}_{\mathrm{n}-10}, \mathrm{C}_{\mathrm{n} 0} \text {. }
\end{aligned}
$$

- Thermal Cycles after Solidification.

The cooling cycle after solidification may be important if the casting spends appreciable time at temperatures where diffusion is significant and the cooling time is long relative to solidification time. Post-solidification cooling may be simulated, as well, during the execution of the CASTSEG module.

- Solution Treatment Temperature(s) and Time(s). 


\section{Section 2: Solutionizing Prediction}

Solution treatment temperature $\left(T_{H}\right)$ and solution treatment time $\left(\theta_{H}\right)$ may be entered directly. The complete thermal cycle for a specific location in the casting, including reheating, hold at (or cycling around) the solution treatment temperature, and cooling between furnace removal and entry to the quenching tank, may be entered as an expression or as tabular data, either from data files or as direct input from thermal prediction software as described in section 1.0.

- Phase Relations in the Solution Treatment Regions.

Input from PHASECALC (See section 2.1).

- Diffusion Coefficients for the Alloying

Input from DIFFCALC See section 2.2).

- Initial Microstructure (phase fractions and distribution, solute distribution, and characteristic dimensions)

Intput from CASTSEG or electronic storage..

2. Computation Control Parameters:

- Thickness and numbering of distance elements $(\Delta \lambda)-1$-D plate-like model.

The characteristic volume element of thickness das/2 is divided into $\mathrm{N}$ distance elements or "slices" (typically, $200<\mathrm{N}<1000$ ). For uniformly spaced elements $\Delta \lambda=$ das $/ 2 \mathrm{~N}$. See Figure 2.3-2.

The slice centered at $\lambda=0$ is numbered $n=0$ and the slice centered at $\lambda=$ das $/ 2$ is numbered $n=N$. Half of slice $n=0$ and $n=N$ is in the characteristic volume element and half of each of these slices is in a neighboring volume element. Slices labeled $\mathrm{n}=\tilde{-}-1$, centered at $\lambda \stackrel{\tilde{=}}{=}-\Delta \lambda$, and $\mathrm{n}=\mathrm{N}+1$, centered at $\mathrm{x}=\mathrm{das} / 2+\Delta \lambda$, are used to implement the symmetry boundary condition.

- Thickness and numbering of distance elements $\left(\Delta \lambda_{1} \times \Delta \lambda_{2}\right)-2$-D plate-like model. 


\section{Section 2: Solutionizing Prediction}

The characteristic volume element is based on two characteristic distances, typically, one-half of the secondary dendrite arm spacing $\left(\mathrm{d}_{2}\right)$ and one-half of the primary dendrite arm spacing $\left(d_{1}\right)$. Note Figure 2.3-10. The number of differential elements taken in each direction is the same. The differential volume elements will be rectangular with the dimensions $\Delta \lambda_{1} \times \Delta \lambda_{2}$ in the same ratio as $\mathrm{d}_{1} \times \mathrm{d}_{2}$. Symmetry boundary conditions will apply at $\lambda_{1}=0 ; \lambda_{2}=0 ; \lambda_{1}=\mathrm{d}_{1} / 2 ; \lambda_{2}=\mathrm{d}_{2} / 2$. The order of solidification of rectangular elements in the 2-D model is not relevant to the execution of CASTSOLN (except as it influences the input initial solute distribution). The iterative diffusion computation, Ficks second law, equation 2.3-5, is extended to two-dimensions.

- Size of time steps $(\Delta t)$ for FDM computation.

Typically a time step for the diffusion computation, $\Delta \mathrm{t}_{\text {Diff, }}$ is selected so that

$$
\frac{D^{\phi} \Delta t_{D i f f}}{\Delta \lambda^{2}}<\frac{1}{8}
$$

\section{$\underline{\text { Solutionizing Simulation Steps for a Binary Alloy System }}$}

1. The current temperature $T^{*}$ is computed from the stored data for the thermal cycle: post-solidification cooling rate or solution treatment time(s) and temperature(s).

2. The solvus composition $\mathrm{C}^{\alpha *}$ for the current $\mathrm{T}^{*}$ is located on the stored solvus line for the primary phase.

3. Compute diffusion in the primary solid phase, in elements $0<\mathrm{n}<\mathrm{n}^{*}$, according to Fick's Second Law, equation 2.3-5, with the boundary conditions

$$
\begin{array}{ll}
\left(\frac{\Delta C^{\alpha}}{\Delta \lambda}\right)_{n=0}=0 & @ \lambda=0 \\
\left(\frac{\Delta C^{\alpha}}{\Delta \lambda}\right)_{n=N}=0 & @ \lambda=L=\frac{\text { das }}{2}
\end{array}
$$




\section{Section 2: Solutionizing Prediction}

$$
\begin{array}{rr}
C_{n}^{\alpha}(t)=\bar{C}_{n}^{\alpha}(t) & \text { for } \quad \bar{C}_{n}^{\alpha}(t)<C^{\alpha *} \\
C_{n}^{\alpha}(t)=C^{\alpha *} & \text { for } \bar{C}_{n}^{\varepsilon}(t)>C^{\alpha *}
\end{array}
$$

where $n *$ represents the slice at the boundary between the single and two phase regions, $\bar{C}_{n}^{\alpha}(t)$ represents the average composition of volume element $\mathrm{n}$ at time $\mathrm{t}, C_{n}^{\alpha}(t)$ represents the solute concentration within the primary phase for slice $\mathrm{n}$ at time $\mathrm{t}$.

The assumption in step 3 is that any element that has an average composition greater than $\mathrm{C}^{\alpha *}$ will be two phase. The two-phase regions can have two origins.

- During solidification the element reaches the eutectic composition and solidifies as the eutectic mixture of phases.

- During solidification the element solidifies at a primary phase composition, which eventually is above the solubility limit for the primary phase, leading to precipitation of the secondary phase.

Within these two phase elements, solute redistribution is rapid and the particles readjust their composition to the equilibrium composition for the local temperature, $C^{\alpha *}$. As a result there is no long-range diffusion within a two-phase region. Elements at the boundary between single and two-phase regions have an average composition somewhat greater than $\mathrm{C}^{\alpha *}$, and become single phase when the average composition drops below $\mathrm{C}^{\alpha *}$.

4. If the thermal cycle is not complete, return to step 1.

Else, proceed to post processing operations.

\section{$\underline{\text { Solutionizing Simulation Steps for a Ternary Alloy System. }}$}

1. The current temperature $\mathrm{T}^{*}$ is computed from the stored data for post solidification cooling rate or solution treatment time(s) and temperature(s). 


\section{Section 2: Solutionizing Prediction}

2. For each volume element $\mathrm{n}$ at time $\mathrm{t}$, determine if its average composition, $\bar{C}_{i n}(t)$ and $\bar{C}_{j n}(t)$, is below the solubility limit at $\mathrm{T}^{*}$ and is within the $\alpha$-primary phase, is within the two-phase region, or is in a three-phase region.

- If the slice is single phase, set $C_{1 n}^{\alpha}(t)=\bar{C}_{1 n}(t)$, set $C_{2 n}^{\alpha}(t)=\bar{C}_{2 n}(t)$, and $f_{n}^{\alpha}(t)=1$.

- If the slice is two-phase, locate the tie line and determine the solubility limits, $C_{1}^{\alpha *}, C_{2}^{\alpha *}, C_{1}^{\beta *}$, and $C_{2}^{\beta *}$, on the $\alpha$-solvus and $\beta$-solvus for this temperature, $\mathrm{T}^{*}$, and average composition, $\bar{C}_{1 n}(t)$ and $\bar{C}_{2 n}(t)$. From the average composition and the compositions of the individual phases compute the fraction of primary phase, $f_{n}^{\alpha}(t)$, within the slice. Set $C_{1 n}^{\alpha}(t)=C_{1 n}^{\alpha} *(t)$. and $C_{1 n}^{\alpha}(t)=C_{2 n}^{\alpha} *(t)$.

- If the slice is three-phase, use the tie triangle to determine the solubility limits, $C_{1}^{\alpha * *}, C_{2}^{\alpha * *}, C_{1}^{\beta * *}, C_{2}^{\beta * *}, C_{1}^{\chi * *}$, and $C_{2}^{\chi * *}$, for this temperature, $\mathrm{T}^{*}$, and average composition, $\bar{C}_{1 n}(t)$ and $\bar{C}_{2 n}(t)$. From the average composition and the compositions of the individual phases compute the fraction of primary phase, $f_{n}^{\alpha}(t)$, within the slice. Then set $C_{1 n}^{\alpha}(t)=C_{2 n}^{\alpha} * *(t)$ and $C_{1 n}^{\alpha}(t)=C_{2 n}^{\alpha} * *(t)$.

3. Compute diffusion in the primary solid phase, in each element $0<\mathrm{n}<\mathrm{N}$ according to Fick's Second Law, equation 2.3-5

4. If the thermal cycle is not complete return to step 1.

Else, proceed to post processing operations.

\section{$\underline{\text { Output in tabular or graphical form }}$}

1. Solute profiles of each component giving either the average concentration or the concentration in individual phases across the characteristic volume element.

2. Fraction of primary and secondary phases after cooling to room temperature and during or after solution heat treatment. 


\section{Section 2: Solutionizing Prediction}

\subsubsection{Application of CASTSOLN to Al-6.5\% Si-3.5\% Cu}

The predictions of the LSD 1-D plate-like model, at the end of solidification, have been used as the initial condition for the simulation of solution heat treatment at three temperatures: $723 \mathrm{~K}$, $753 \mathrm{~K}$, and $778 \mathrm{~K}$. See Figure 2.4-1. According to the phase diagram data used, the solubility of $\mathrm{Cu}$ at these temperatures is $3.04 \%, 4.11 \%$, and $4.3 \%$, respectively. During solution heat treatment, it is possible to dissolve all of the $\theta$-phase in an $\mathrm{Al}-6.5 \% \mathrm{Si}-3.5 \% \mathrm{Cu}$ alloy at the higher two temperatures. About $30 \%$ of the $\theta$-phase in the as-cast alloy is an equilibrium constituent and will not dissolve at $723 \mathrm{~K}$.

The symbol $\theta_{O}$ is used to represent the weight fraction of $\theta$-phase in the as-cast alloy. The relative fraction of $\theta$-phase remaining is plotted as a function solution treatment time in Figures 2.4-1 for three values of as-cast dendrite arm spacing. Using the parabolic relation between diffusion time and diffusion distance, the results of the simulation are presented in a more compact form in Figure 2.4-2. The relative fraction of $\theta$-phase remaining is plotted as a function of the solution treatment time divided by the square of one-half the dendrite arm spacing and the data reduce to a single curve for each temperature.

Figure 2.4-3 compares for the 1-D and 2-D plate-like models the reduction in $\theta$-phase predicted for solution treatment at $505^{\circ} \mathrm{C}$ for an $\mathrm{Al}-7 \% \mathrm{Si}-3.5 \% \mathrm{Cu}-0.5 \% \mathrm{Mg}$ alloy with a dendrite arm spacing of $38 \mu \mathrm{m}$. The 2-D model was developed because of the observation that $\theta$-phase in the interdendritic areas between secondary arms dissolved quickly during solution treatment. On-the-other-hand clusters of $\theta$-phase at primary arm boundaries and at grain boundary junctions took much longer to dissolve. The 2-D plate-like model, based on two characteristic spacings, is a better representation of the overall dissolution kinetics of $\theta$-phase in ternary Al-Si-Cu alloy.

\section{Application of CASTSOLN to Al-7\%Si-3.5\% Cu-0.5\%Mg}

Figure 2.4-4 illustrates the application of CASTSOLN on the solution treatment at $505^{\circ} \mathrm{C}$ of an $\mathrm{Al}-7 \% \mathrm{Si}-3.5 \% \mathrm{Cu}-0.5 \% \mathrm{Mg}$ alloy. The predicted $\mathrm{Cu}$ and $\mathrm{Mg}$ distributions in the $\alpha$-phase are plotted versus solutionizing times from $1 / 2$ to 16 hours. Note the $\mathrm{Mg}$ and $\mathrm{Cu}$ distributions are 


\section{Section 2: Solutionizing Prediction}

fairly uniform after $1 / 2$ hour of solution treatment. The scales on both plots have small differences in composition. Some change in the copper content of the $\alpha$-phase continues for 16 hours as a result of the continual dissolution of $\theta$-phase. The final $\mathrm{Cu}$ concentration of the $\alpha$-phase exceeds the nominal alloy composition. The silicon-phase fraction that remains insoluble contains negligible $\mathrm{Cu}$. The $\mathrm{Mg}$ concentration in the $\alpha$-phase is below the nominal alloy composition. Not all of the Q-phase is soluble, and the remaining $\mathrm{Mg}$ is contained in the equilibrium fraction of Q-phase.

Figure 2.4-5 illustrates the rate of dissolution of $\theta$-phase and Q-phase for solution treatment of the quaternary alloy at $480^{\circ} \mathrm{C}$. All of the $\theta$-phase will eventually dissolve. About $0.35 \mathrm{vol} \%$ Q-phase is stable at this temperature and overall alloy composition. The major dissolution of both intermetallic phases occurs within the first 2 hours. Dissolution of $\theta$-phase, in particular, continues at ever decreasing rate beyond 16 hours. The $\theta$-phase contains about $50 \mathrm{wt} \% \mathrm{Cu}$. The dissolution of even a small amount of $\theta$-phase results in a significant increase in the $\mathrm{Cu}$ content of the $\alpha$-phase. 


\section{Section 2: Solutionizing Prediction}
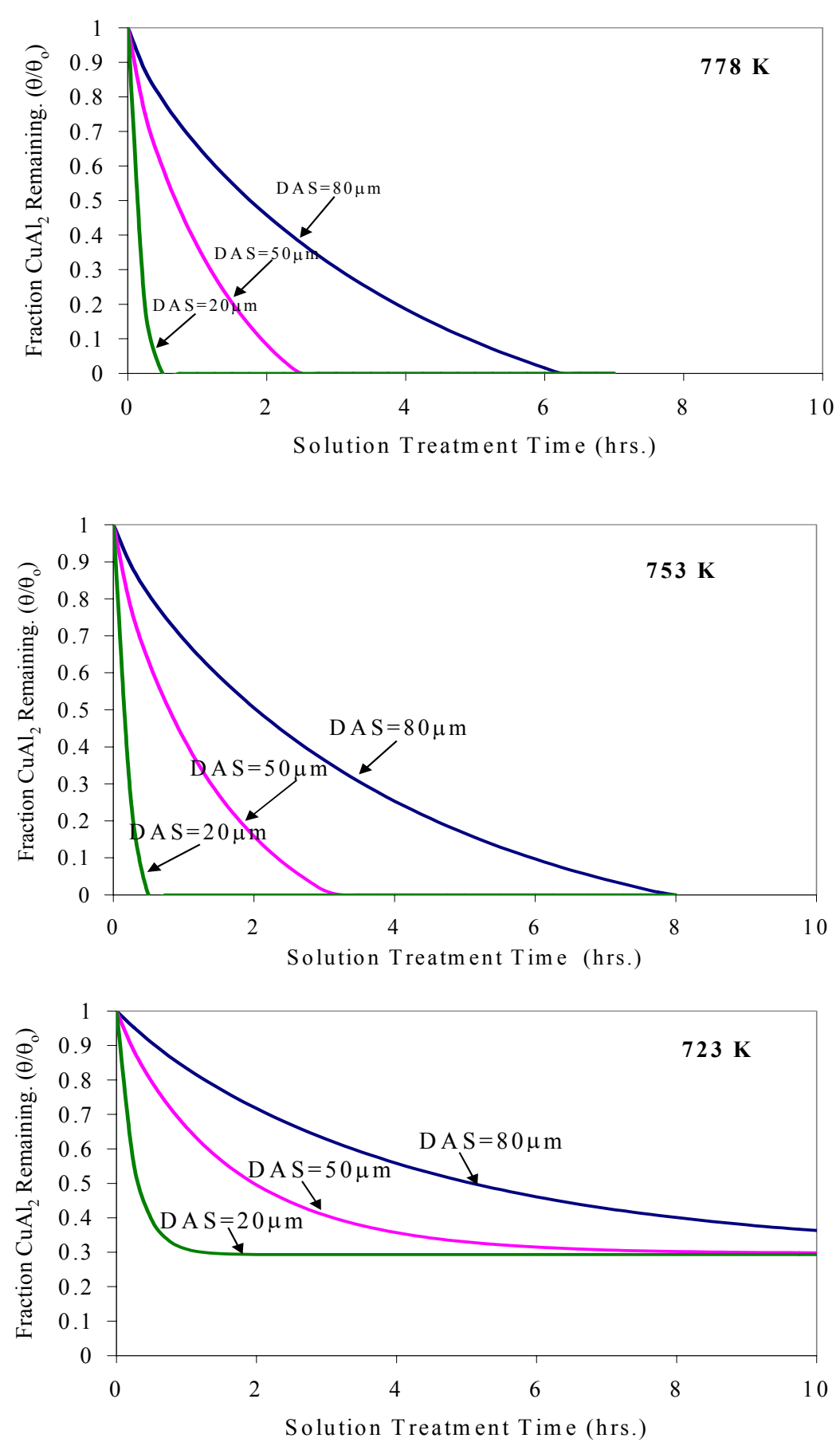

Figure 2.4-1. Relative rate of dissolution of $\mathrm{CuAl}_{2}$ at three solution treatment temperatures and for three as-cast dendrite arm spacings in $\mathrm{Al}-6.5 \% \mathrm{Si}-3.5 \% \mathrm{Cu}$ alloy 


\section{Section 2: Solutionizing Prediction}

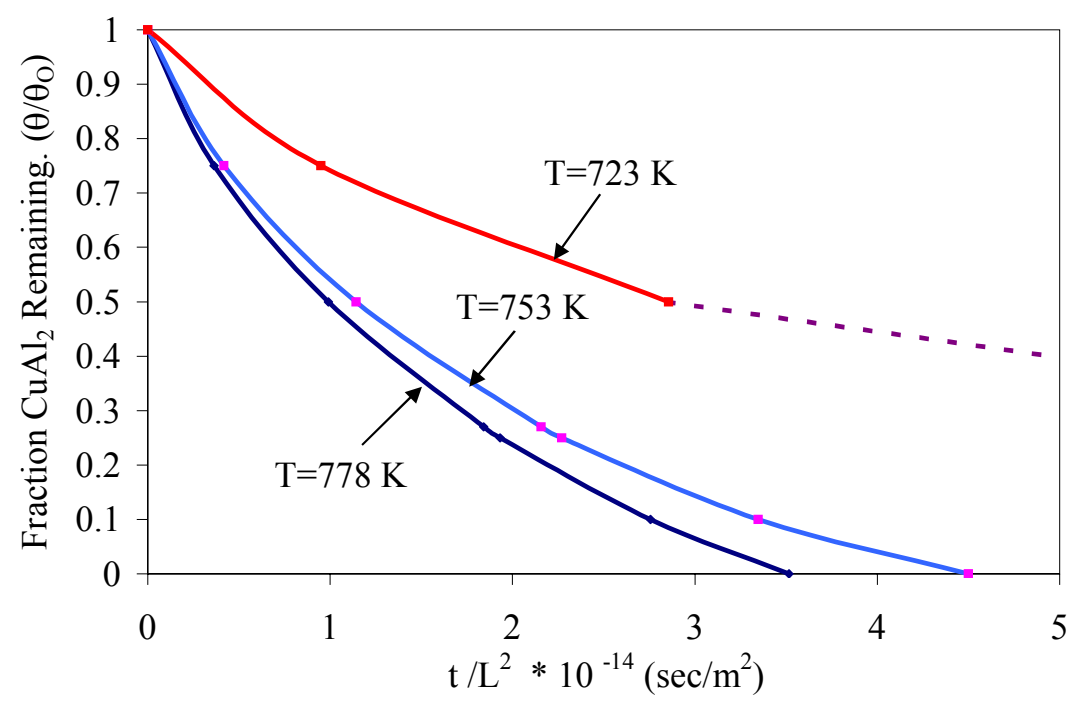

Figure 2.4-2. Rate of dissolution of $\theta$-phase in Al-6.5\% $\mathrm{Si}-3.5 \% \mathrm{Cu}$ alloy plotted for three solution treatment temperatures as a function of solution treatment time divided by half of the dendrite arm spacing squared

Weight Percent of $\theta$-phase during Solution Treatment (Al-7\%Si-3.5\%Cu, DAS=38 m, T=778 K)

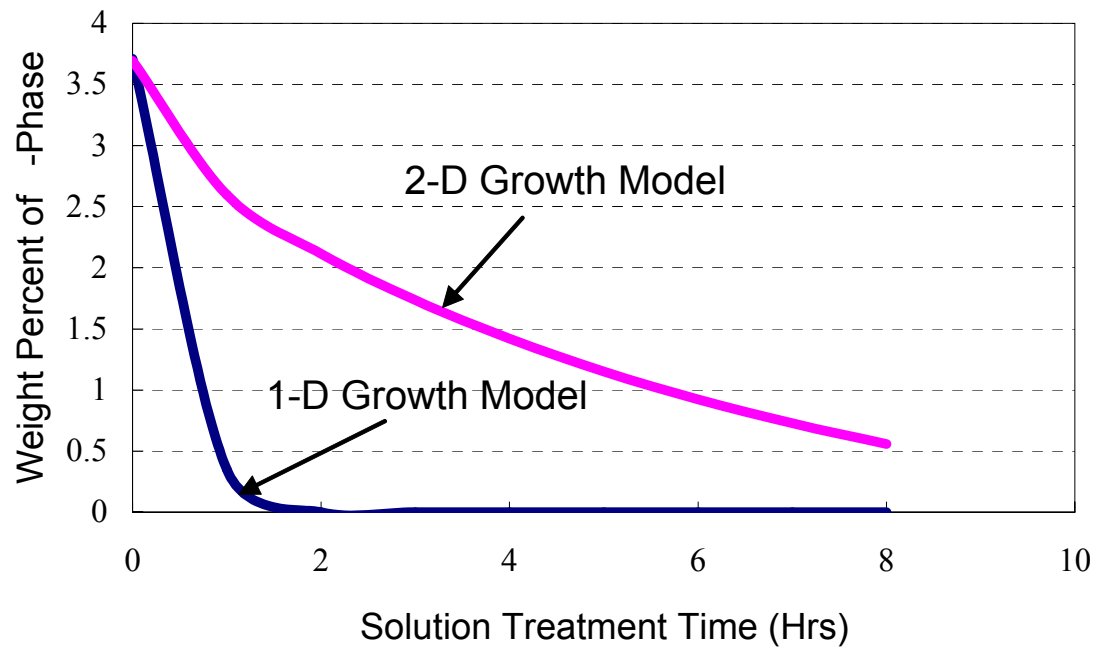

Figure 2.4-3. Dissolution of $\theta$-phase during solution treatment of $\mathrm{Al}-7 \% \mathrm{Si}-3.5 \% \mathrm{Cu}$ at $505^{\mathrm{O}} \mathrm{C}$ : Comparison of 1-D and 2-D models 


\section{Section 2: Solutionizing Prediction}

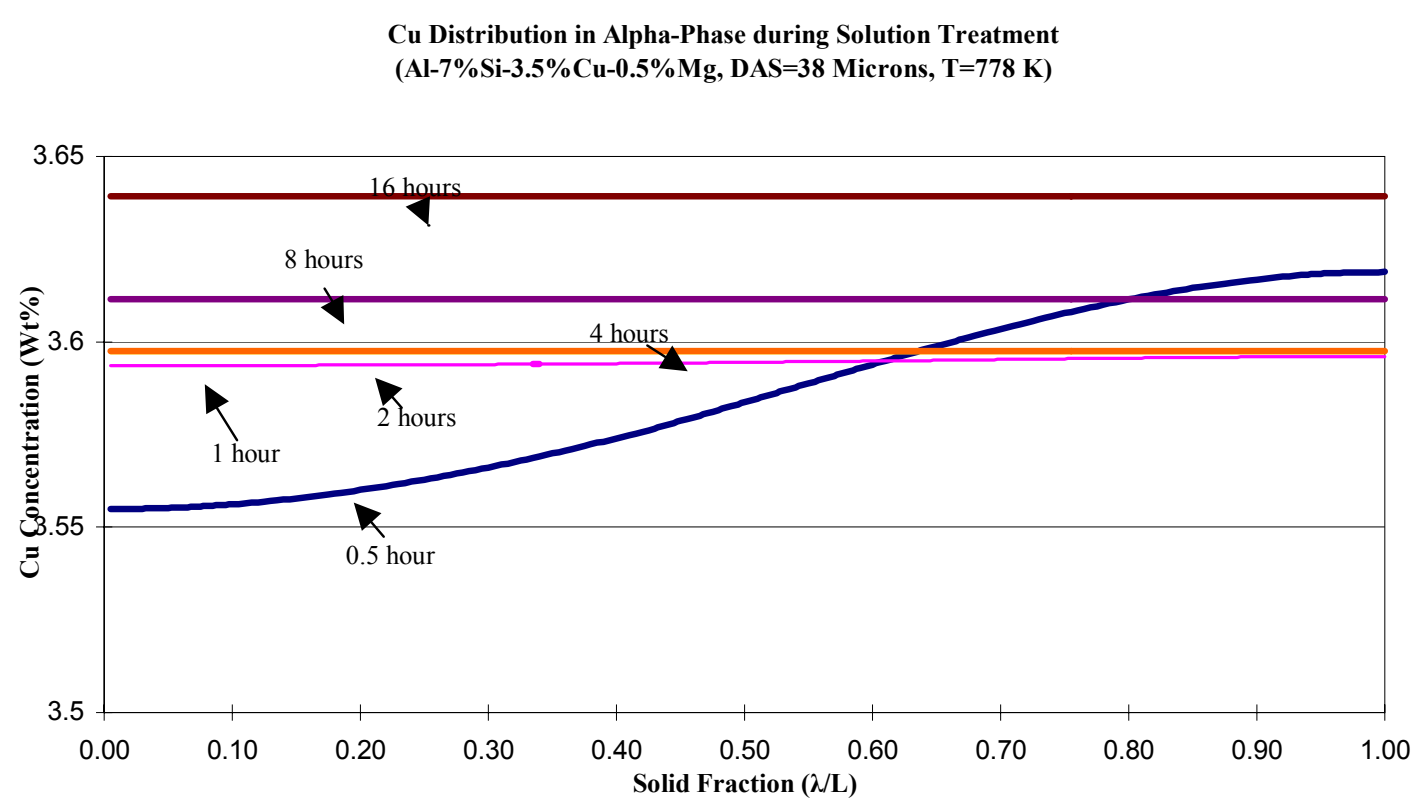

Mg Distribution in Alpha-Phase during Solution Treatment (Al-7\%Si-3.5\%Cu-0.5\%Mg, DAS=38 Microns, T=778 K

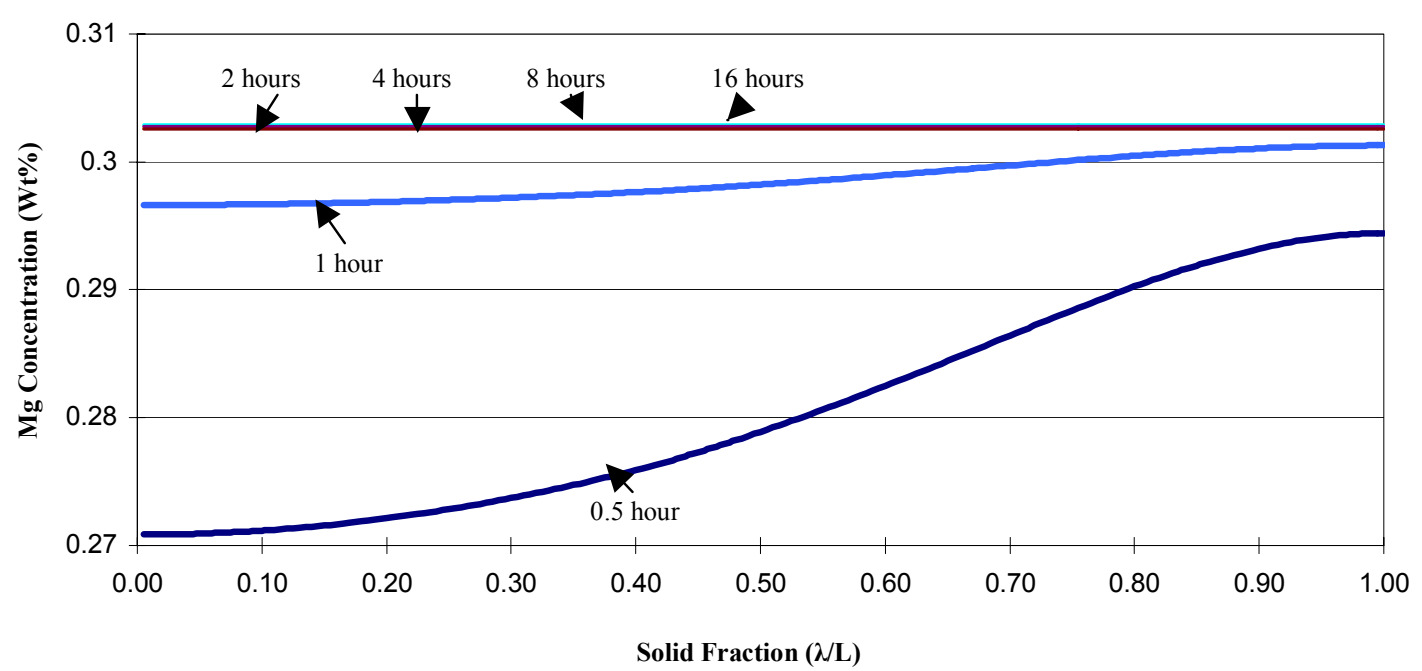

Figure 2.4-4. Copper (top) and magnesium (bottom) distributions in the $\alpha$-phase for a quaternary $\mathrm{Al}-7 \% \mathrm{Si}-3.5 \% \mathrm{Cu}-0.5 \% \mathrm{Mg}$ alloy after several solution treatment times at $505^{\mathrm{O}} \mathrm{C}$ 


\section{Section 2: Solutionizing Prediction}

Volume Fraction of Q-Phase during Solution Treatment

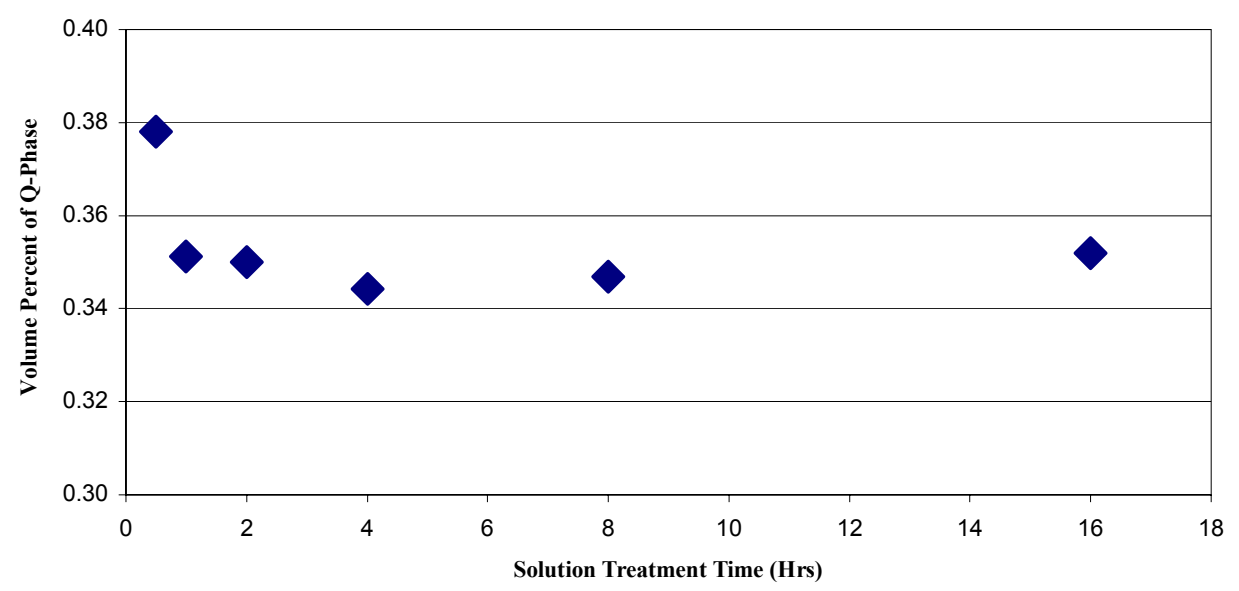

Volume Fraction of $\theta$-Phase during Solution Treatment

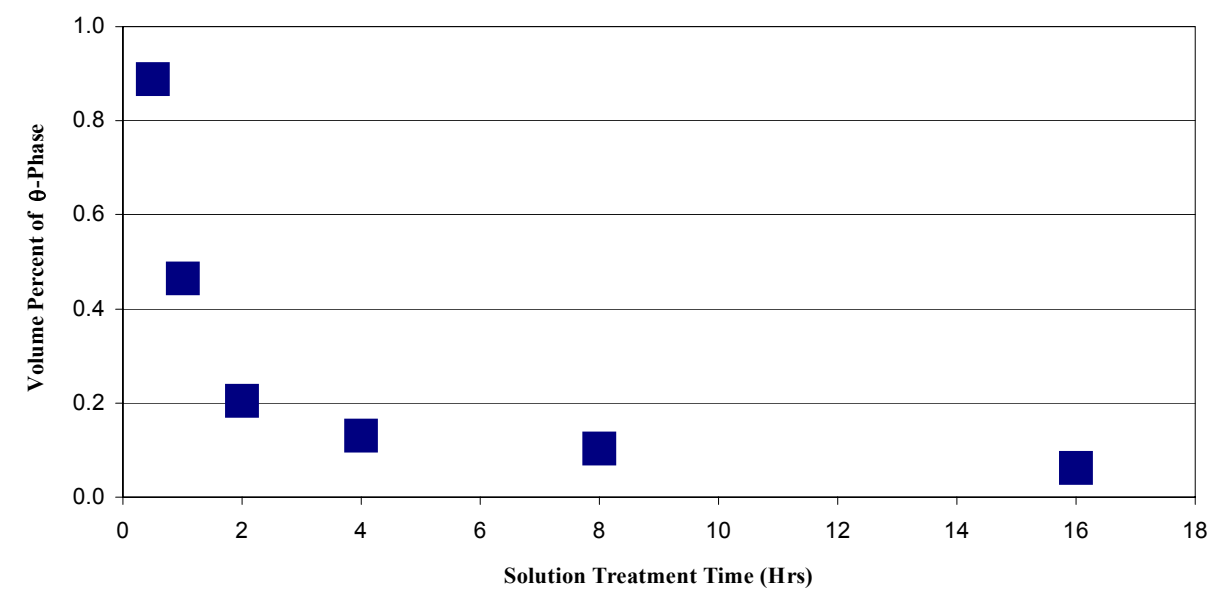

Figure 2.4-5. Rate of dissolution of Q-phase (top) and $\theta$-phase (bottom) for quaternary alloy solution treated at $480^{\circ} \mathrm{C}$ 


\section{Section 3: Databases}

The databases have been developed by mining the existing literature and by selected experimentation. The data mined from literatures are compared with each other and analyzed, and some are also compared with the experimental results.

The databases in the project are classified into following databases:

- Heat transfer databases:

o Material database;

o Atmosphere database;

o Fuel/gas database;

- Quenching performance database;

- Furnace database;

- Phase diagram for a selected alloy system (Al-Si-Cu);,

- Diffusivity versus composition and temperature;,

- Properties versus porosity, Si particle size, undissolved $\theta$ and grain size.

\subsection{Heat transfer databases}

Databases of thermal properties of aluminum have been preliminary established. One is for the HTFURNCE module, the other one is QuenchPAD, QuenchBIB, and Quench Miner for quenching.

\subsubsection{Material database}

The database consists of density, specific heat, thermal conductivity, emissivisty. They are the function of temperature. And emissivity is also the function of surface finish of the material. Database management has been developed. The addition, edition and removal of the data are achieved.

\section{1) Database structure}




\section{Section 3: Databases}

The material database includes material type, material name, surface status, temperature scale, density, thermal conductivity, specific heat and emssivity. Among them, density, thermal conductivity and specific heat are functions of temperature, while emissivity is the function of temperature and surface status of the material as well. The structure of material database and relations of the items are shown in Figure 3.1-1.

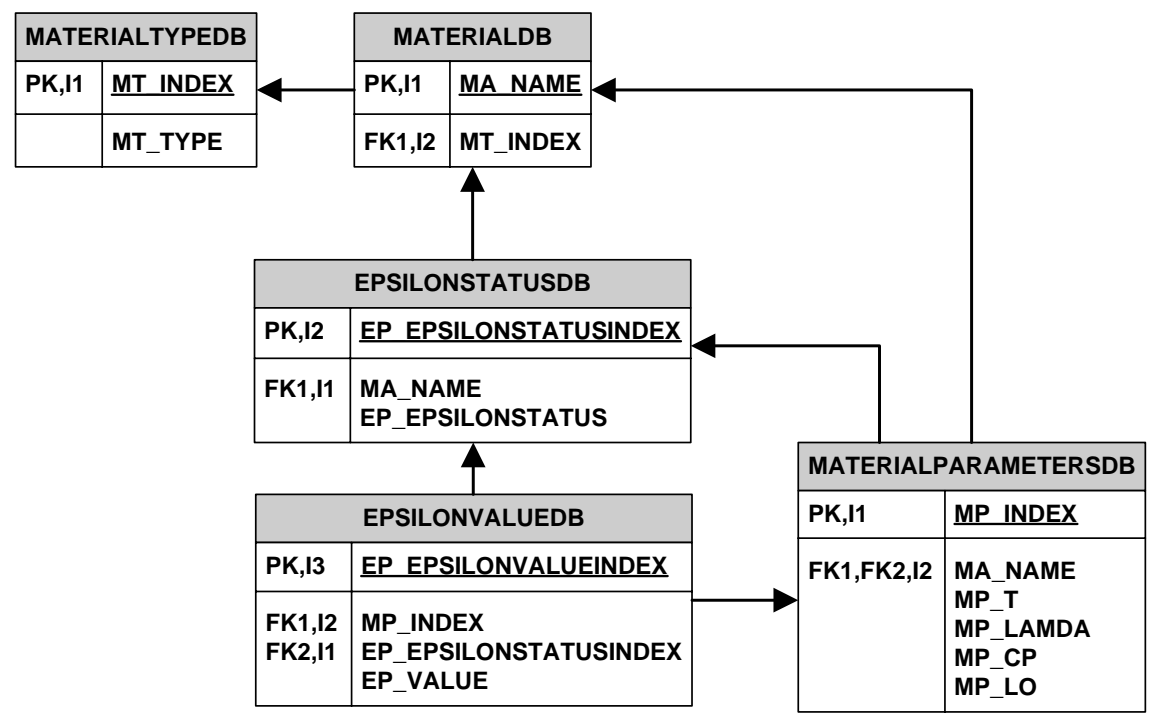

Figure 3.1-1. Material database structure

\section{2) Database management}

Five tables are linked together for the material database - material type table, material table, material parameter table, material surface status definition table, material emissivity table. All of the material database management interfaces are illustrated below.

The material database includes 12 main material categories and 2000 materials. The aluminum alloys are as follows:

- Cast Aluminum: Total: 49

- Wrought Aluminum: Total: 59 


\section{Section 3: Databases}

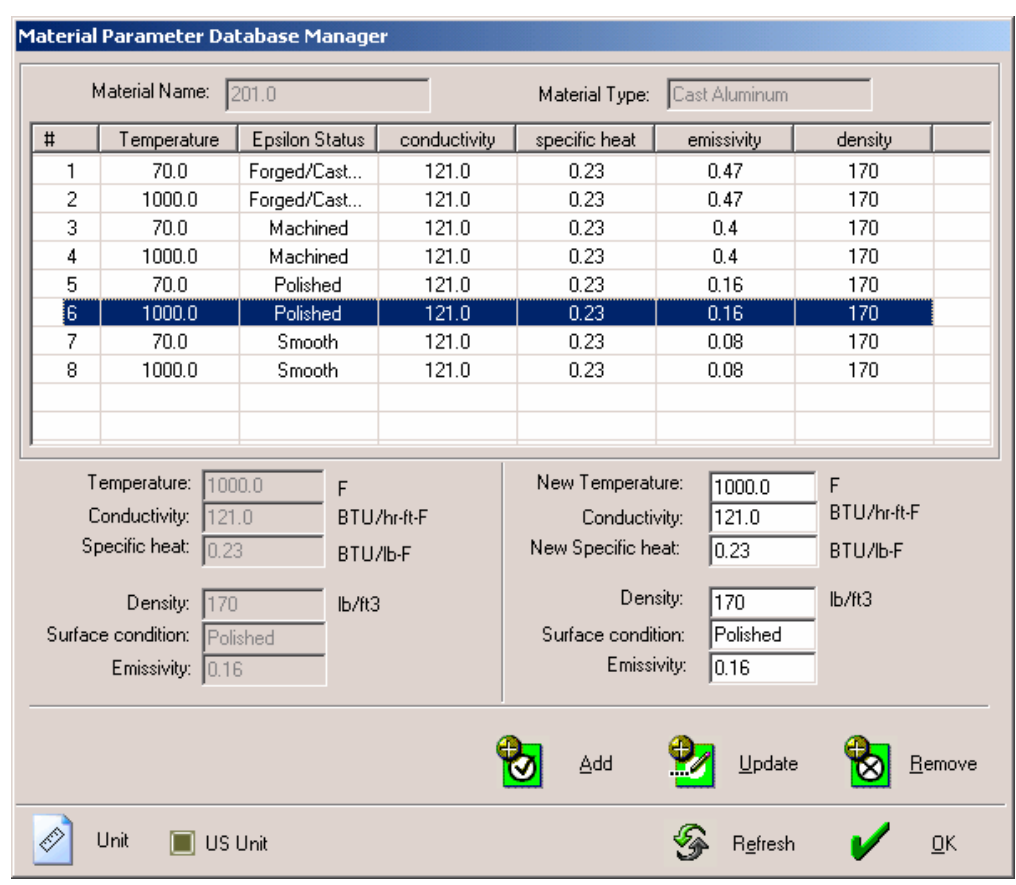

Figure 3.1-2. Material database management

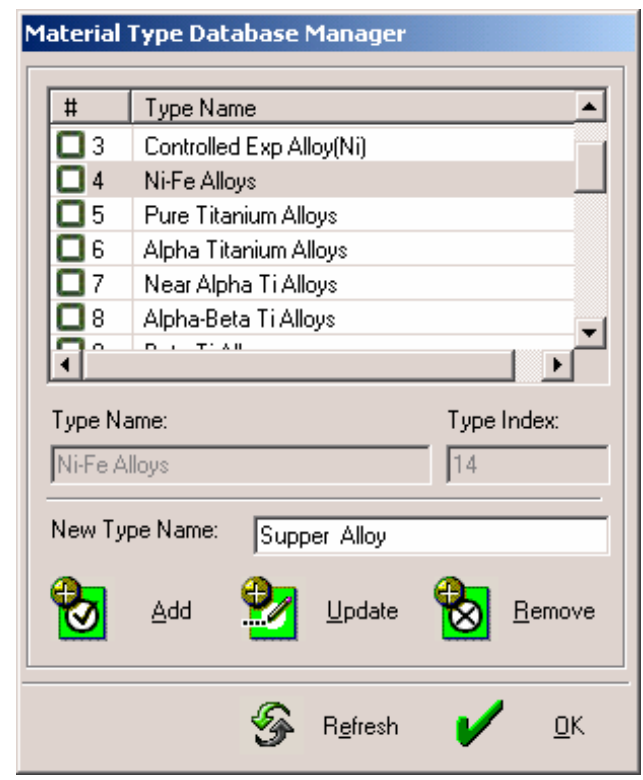

Figure 3.1-3. Material type management

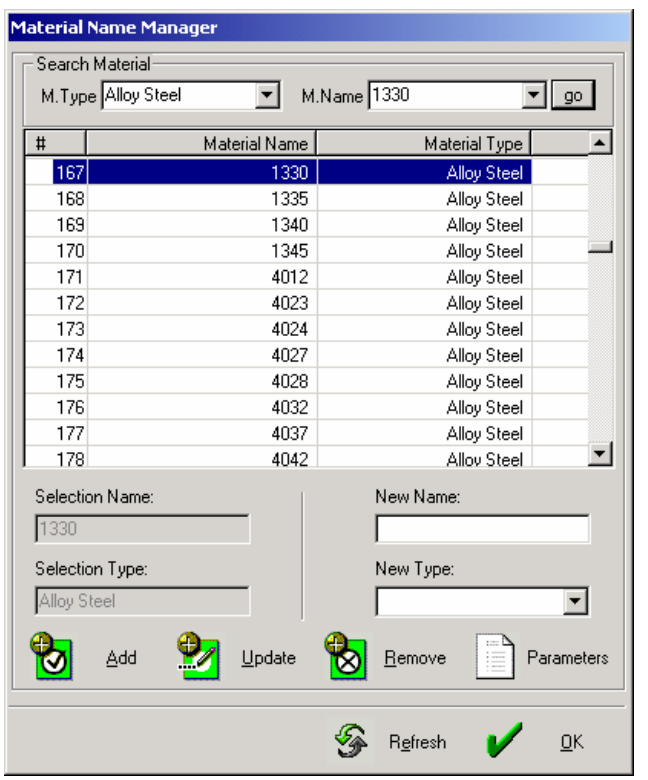

Figure 3.1-4. Material name management 


\section{Section 3: Databases}

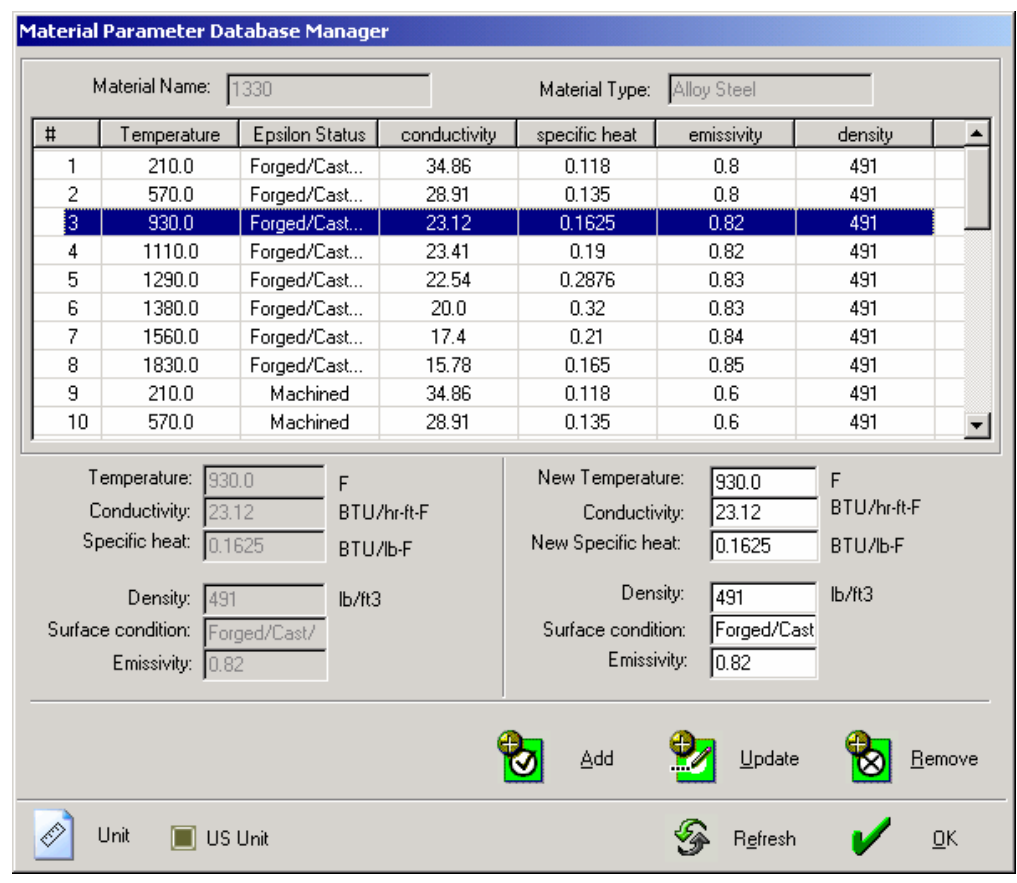

Figure 3.1-5. Material thermal properties management 


\section{Section 3: Databases}

\subsubsection{Atmosphere database}

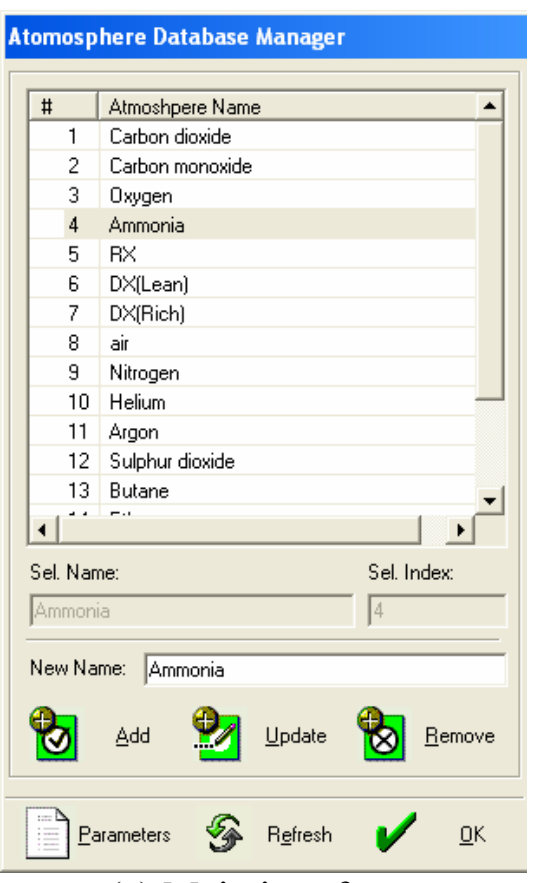

(a) Main interface

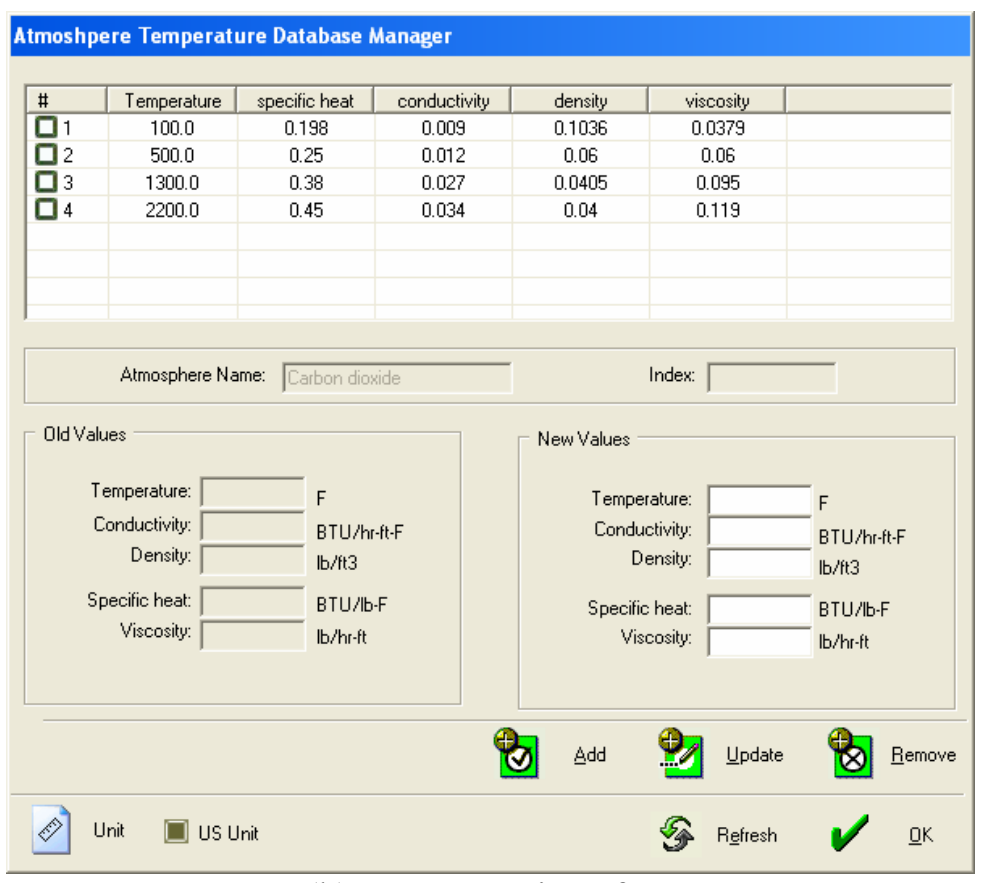

(b) Parameter interface

Figure 3.1-6. Atmosphere database management

Density, thermal conductivity, specific heat, and static viscosity data are specified for the atmosphere in the database. The management interface is shown in figure 3.1-6.

\subsubsection{Fuel/gas database}

The fuel database manages heat content, fuel/air ratio, constants for available heat calculation data for fuel/gas. The database management interface is shown below. 


\section{Section 3: Databases}

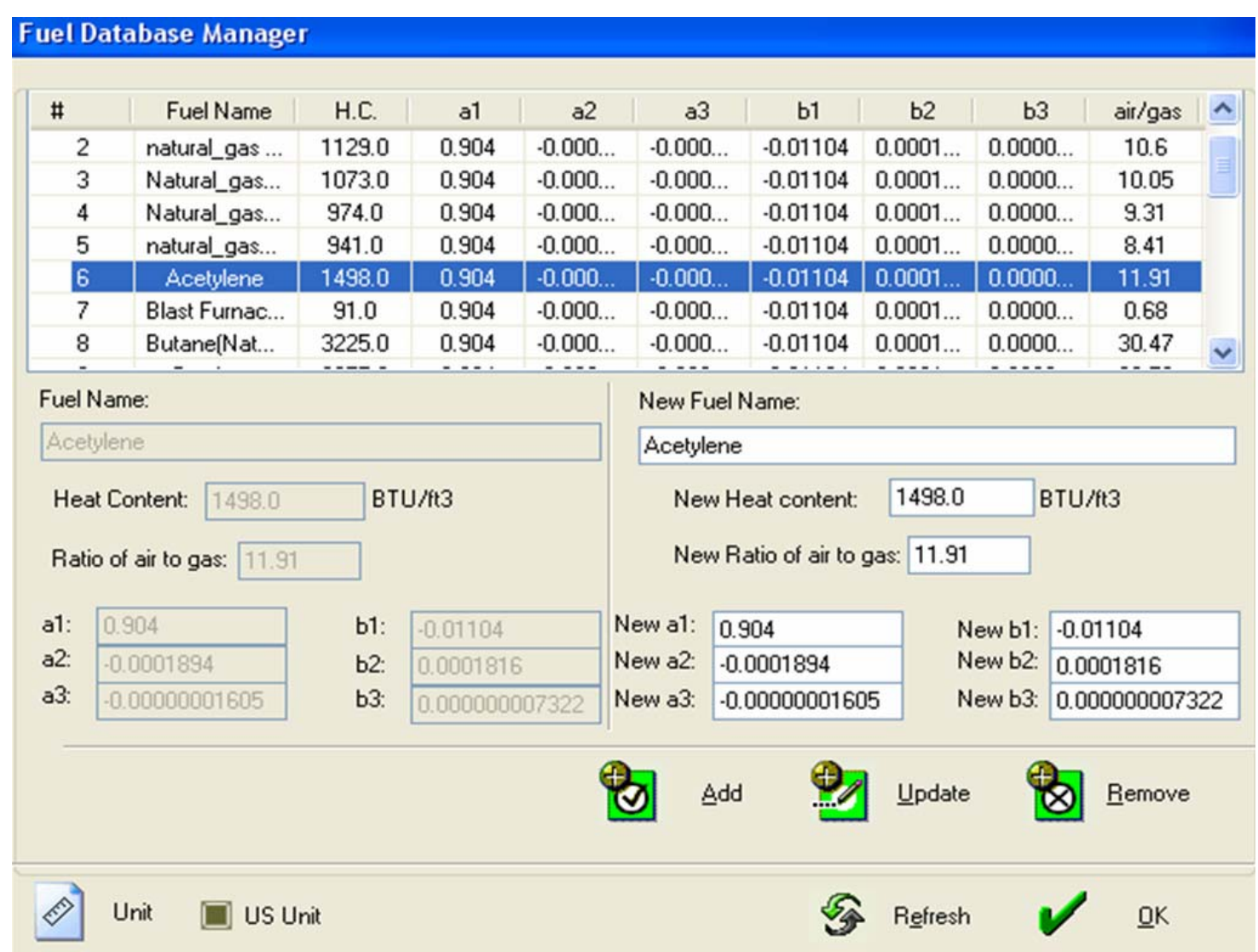

Figure 3.1-7. Fuel database management

\subsection{Furnace database}

From the furnace database, a 3D furnace model can be re-constructed by furnace basic data (external size, workspace, power, gross load weight, operating temperature, heating type), recirculation fan data, furnace wall data, accessories data. Therefore in the calculation the furnace CAD model is used directly so as to simplify the calculation and improve efficiency. In figure 3.2-1, the furnace database management interface is plotted. 


\section{Section 3: Databases}

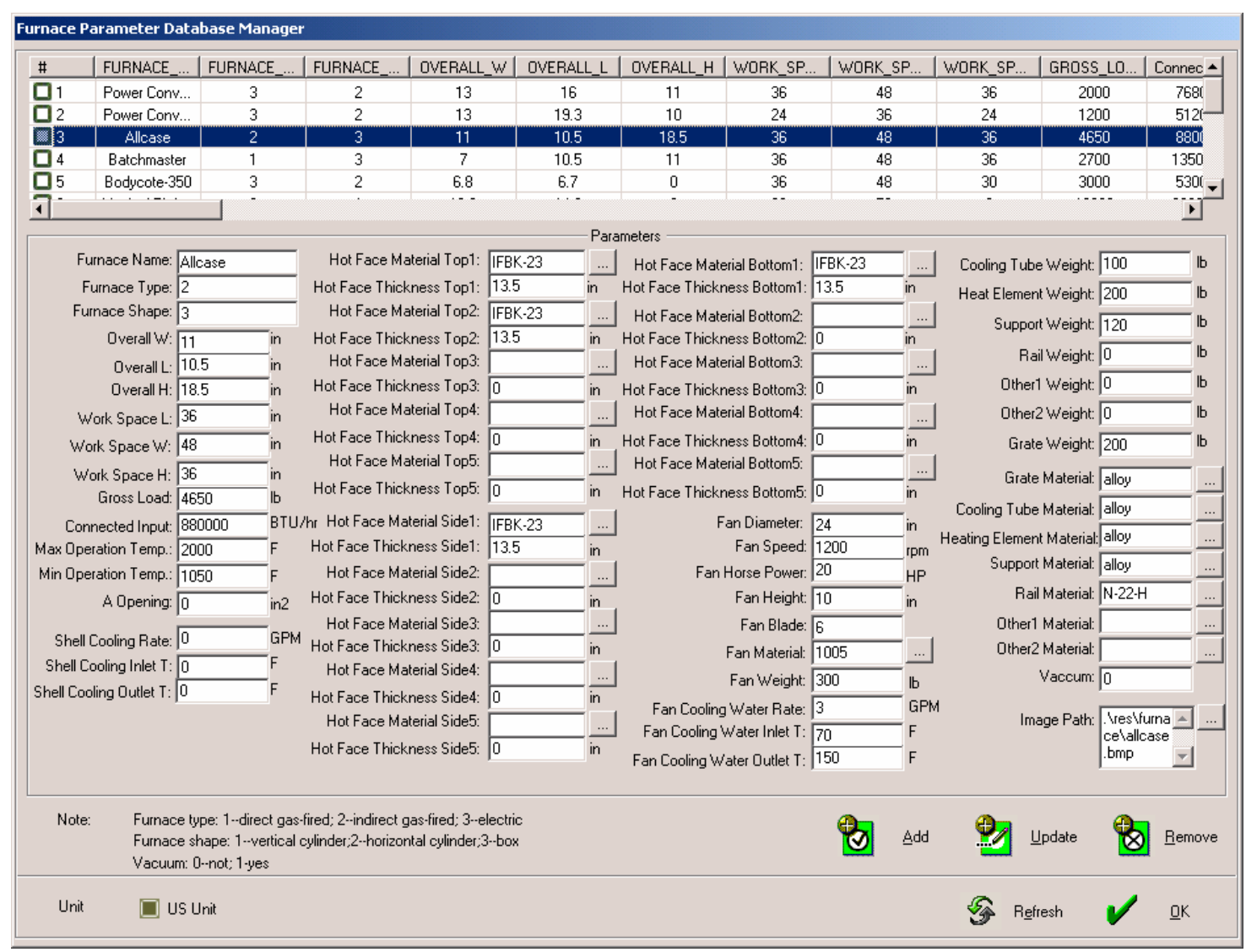

Figure 3.2-1. Furnace database management

\subsection{Database of Heat Transfer Coefficient ---QuenchPAD and QuenchMiner}

A Database System called QuenchPADTM, (Quenchant Performance Analysis Database) has been developed to store mostly quenching related textual and numerical data in a relational format using commercial package MS Access. The Database system manages the quenching information keeping it up-to-date with changes, and running the user's queries.

A tool, QuenchMiner ${ }^{\mathrm{TM}}$ is also developed that makes the features of QuenchPAD ${ }^{\mathrm{TM}}$ available on the World Wide Web, and in addition, it also captures graphical, statistical and other complex 


\section{Section 3: Databases}

data in relational formats. Moreover, it also supports decision making. It allows the users to submit certain scenarios and suggests solutions based on current information, history data and domain knowledge.

\subsection{Phase Equilibria Database}

The objective of this task is to gather equilibrium phase relations for multi-component multiphase aluminum alloys in the temperature ranges and composition ranges relevant to the solidification and solution heat treatment of aluminum alloy castings, especially for automotive applications. A parallel objective is to validate the models developed for simulation of the behavior of multicomponent multiphase microstructure evolution and solute redistribution during solidification and heat treatment processes. By iteratively comparing the results of experiments and the predictions of models, our understanding of the phenomena that underlie the models, the reliability of the databases, and the effectiveness of the simulation routines to implement the models are validated.

Emphasis is on the Al-Si-Cu and Al-Si-Mg ternary systems, Al-Si-Cu-Fe and Al-Si-Cu-Mg quaternary systems, and, potentially, the $\mathrm{Al}-\mathrm{Si}-\mathrm{Cu}-\mathrm{Fe}-\mathrm{Mg}$ quinary system. The primary composition ranges of interest are $6-9 \% \mathrm{Si}, 2.5-4.5 \% \mathrm{Cu}, 0.2-0.7 \% \mathrm{Fe}$, and $0.15-0.5 \% \mathrm{Mg}$. The approaches are to search the published literature, to mine available databases, including thermodynamic databases, and directed experimentation. The manifold experimental approach is described in the paragraphs below.

Figure 3.4-1 illustrates the phase equilibrium data that are required by the CASTSEG and CASTSOLN modules for simulation of solidification and solution treatment. The PHASECALC module (section 2.1) accesses the phase equilibrium data. For a quaternary $\mathrm{Al}-\mathrm{Si}-\mathrm{Cu}-\mathrm{Mg}$ alloy of the 319 type, the tie lines in the two-phase region connect liquid and $\alpha$-phase compositions that are in equilibrium. Four neighboring liquid composition points form a tetrahedron used to approximate the liquidus (3-D) surface. Four matching solid compositions form a tetrahedron enclosing the solid compositions on the solidus surface that are 


\section{Section 3: Databases}

in equilibrium. Tie triangles connect the liquid, $\alpha$-phase, and silicon-phase compositions that can be in equilibrium. The surfaces of two-fold saturation are approximated by flat tie triangles. The tie lines in the four-phase region connect four lines of three-fold saturation. The added phase is the Q-phase. The five-phase region is the quaternary eutectic. The added phase is $\mathrm{CuAl}_{2}$, the $\theta$-phase. The temperature and the five phases in the quaternary eutectic are invariant.

\section{$\underline{\text { 3.4.1 Validation Experiments }}$}

Optical metallography, quantitative microscopy, and scanning electron microscopy (SEM) have been applied to the analysis of the microstructures, phase distributions, and solute distribution in aluminum alloy castings for automotive applications. Analyses of industry castings guided the range of process and alloy parameters to be understood, modeled, and for which databases would be assembled. A series of test plates of the alloys of interest were cast and heat-treated with the range of process and alloy parameters important to industry practice.

3.4.1.1 Analysis of Automotive Castings The microstructure of several commercial automotive Al-319 and Al-356 castings have been examined in detail to establish the breadth of industry practices to be modeled by laboratory experiments and computer simulation.. Figure 3.4-2 is an example of the microstructure of a cast and heat-treated commercial aluminum alloy (319). The white dendritic matrix is the terminal solid solution $\alpha$-phase that is discolored by fine precipitates. The blue phase is the silicon-phase that solidified together with $\alpha$-phase to form the binary eutectic microconstituent. The brown particles are iron-rich intermetallic particles. Any residual Q-phase and $\theta$-phase particles are not readily visible at this magnification.

Two scanning electron microscopy images of sections cut from Al-319 cast automotive components are presented in Figure 3.4-3. X-ray spectra are used to identify constituent phases. The measurements of composition, shown on the images, are only semi-quantitative. The volume of the sample energized by the incident electron beam includes both the particle and the neighboring matrix. Measurements made of the matrix also include X-rays emitted by 


\section{Section 3: Databases}

constituent particles below the surface. Nonetheless, the particles can be identified and their morphology characterized so the constituents can be identified, subsequently, by appearance.

3.4.1.2 Test Plate Castings Considerable data on phase equilibria are determined from measurements on the series of test plate castings described in section 3.4. The nominal compositions of the test plates are $\mathrm{Al}-7 \% \mathrm{Si}-3.5 \% \mathrm{Cu}-0.33 \% \mathrm{Mg}-0.5 \% \mathrm{Fe}$ and $\mathrm{Al}-7 \% \mathrm{Si}-0.33 \% \mathrm{Mg}$. Thermal arrests in cooling curves measured at several positions in the plates, provided the onset temperature of solidification phase changes. Quantitative metallographic analyses of coupons cut at several locations from the plate castings provide information on phase identification and amount. A scanning electron microscope image of a section from a plate casting is shown in Figure 3.4-4.

Solute distribution after casting and solution treatment have been collected on the micro-scale by scanning electron microscopy (SEM) and electron beam microprobe analysis (EBMA). SEM analyses are only semiquantitative. EBMA measurements performed with standards provide reliable data that can be compared, quantitatively, with the predictions of simulations. Samples for electron beam microprobe measurements were brought to the University of Massachusetts for analysis.

Solute distributions can be measured by line scans (continuous or point to point) over interesting features. The line scans, however, provide data for a small selected volume only. To obtain statistically reliable solute distributions representative of a characteristic volume measurements are made over a grid of analysis points. Figure 3.4-5 illustrates a grid of measurement points taken so that the spacing is not an integral multiple of the dendrite arm spacing. For a binary alloy the measured solute concentrations are ordered in ascending order. Then the measured solute concentrations are plotted versus the ratio of the point number to the total number of points. Figure 3.4-6 is an example of a measured copper concentration. For multicomponent and multiphase alloys, devolution of a grid of measurements to obtain a multiple solute profiles, including multiphase regions, requires tedious manipulation of the data. 


\section{Section 3: Databases}

3.4.1.3 Diffusion Couples Diffusion couple measurements, on binary, ternary, and quaternary compositions, are described in section 3.5. These samples are held at temperature for a few to several days. Electron beam microprobe analyses of the composition of phases in these long annealed samples provide data on tie lines and tie triangles in the solution treatment temperature range.

\subsubsection{Interrupted Solidification and DTA}

Two related experiment are aimed to directly yield phase equilibria data. Approximately $0.5 \mathrm{~kg}$ samples are slow cooled from the liquid at a constant rate of heat extraction. The sample, which has high thermal conductivity, is surrounded completely by a very good thermal insulator. The temperature of the convection furnace is controlled to maintain a constant temperature difference between the sample and the furnace air. Essentially the entire temperature drop is between the inner insulation wall and the furnace air. The rate of heat extraction from the alloy is directly related to the temperature difference, which is constant. Thermal arrests on the cooling curve measured at the center of the sample provide very distinct changes in slope, which mark the onset of phase transformations. The length of time of cooling through a temperature range can be related directly to the enthalpy for the transformation. Figure 3.4-7 is an example of a cooling curve. The curve at the bottom is the derivative of the cooling curve. Thermal arrests are more apparent on the derivative curve.

The second type of experiment aimed directly to yielding phase equilibria is interrupted solidification. Several $0.1 \mathrm{~kg}$ samples are placed in the convection furnace and equilibrated at a temperature above the liquidus. Then the samples are cooled at a preset rate. Samples are removed quickly from the furnace and water quenched at several temperatures below the liquidus. Metallographic analyses show which phases formed at temperatures above the quench, i.e., those with a coarse microstructure, and those that formed after the quench, i.e., those with a fine microstructure. Electron beam microprobe analyses indicate the composition of the solid phases, at or near their temperature of formation. 


\section{Section 3: Databases}

Figure 3.4-8 is an example of a sequence of quenched microstructures for the nominal quinary alloy. The sequence of phase formation in the captions to Figure 3.4-8 are representative of normal permanent mold and sand mold cooling rates except for the peritectic conversion of $\mathrm{Al}_{5} \mathrm{FeSi}$ to $\mathrm{Cu}_{2} \mathrm{FeAl}_{7}$. At typical casting cooling rates this diffusion controlled process is suppressed. Conversion begins during solution treatment. 


\title{
Section 3: Databases
}

\section{Phase Equilibrium Data --- Quaternary System}

\author{
Example: Al-Si-Cu-Mg
}

Phase Data --- Tie Figures (Interpolation Shape)

Two Phase Region --- Tie Lines (Tetrahedra)

$$
T_{i}: C_{S i i}^{L}, C_{C u i}^{L}, C_{M g i}^{L}: C_{S i i}^{\alpha}, C_{C u i}^{\alpha}, C_{M g i}^{\alpha}
$$

\section{Three Phase Region --- Tie Triangles (Surfaces)}

$$
T_{j}: C_{S i j}^{L} C_{C u \rho}^{L} C_{M g j}^{L}: C_{S i j}^{\alpha} C_{C u \rho}^{\alpha} C_{M g j}^{\alpha}: C_{S i j}^{\text {silicon }}, C_{C u j}^{\text {silicon }}, C_{M g j}^{\text {silico }}
$$

\section{Four Phase Region --- Tie Tetrahedra (Lines)}

$$
\begin{gathered}
T_{k}: C_{S i k}^{L}, C_{C u k}^{L}, C_{M g k}^{L}: C_{S i k}^{\alpha}, C_{C u k}^{\alpha}, C_{M g k}^{\alpha}: C_{S i k}^{\text {silicon }}, C_{C u k}^{\text {silicon }}, C_{M g k}^{\text {silicon }}: C_{S i k}^{Q}, C_{C u k}^{Q}, C_{M g k}^{Q} \\
\text { Five Phase Region - - Quaternary Eutectic } \\
T_{l}: C_{S i p}^{L} C_{C u P}^{L} C_{M g l}^{L}: C_{S i p}^{\alpha} C_{C u p}^{\alpha} C_{M g l}^{\alpha}: C_{S i l}^{\text {silicon }}, C_{C u l}^{\text {silicon }}, C_{M g l}^{\text {silicon }}: C_{S i p}^{Q} C_{C u P}^{Q} C_{M g l}^{Q}: C_{S i p}^{\theta} C_{C u P}^{\theta} C_{M g i}^{\theta}
\end{gathered}
$$

Figure 3.4-1. Equilibrium tie line data for a quaternary alloy system stored for access by PHASECALC. Order of phase appearance on cooling the liquid is $\alpha$-phase aluminum rich terminal solid solution, silicon, $\mathrm{Mg}$ and $\mathrm{Cu}$ rich Q-phase, and $\mathrm{CuAl}_{2}, \theta$-phase 


\section{Section 3: Databases}

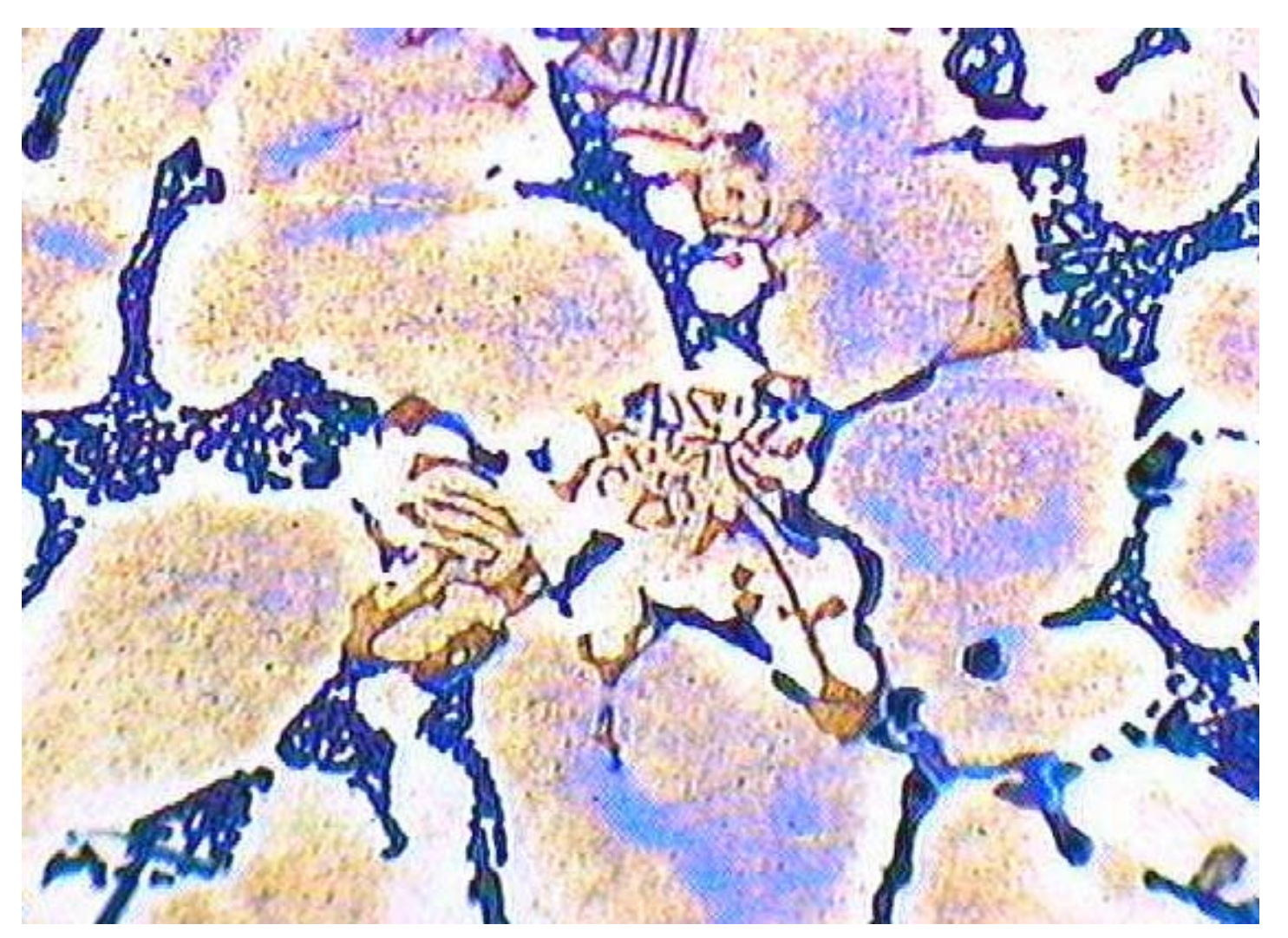

Figure 3.4-2. Microstructure of cast and heat-treated alloy 319 from an automotive component. The color etch turns the silicon particles blue and the iron-rich intermetallic brown 


\section{Section 3: Databases}

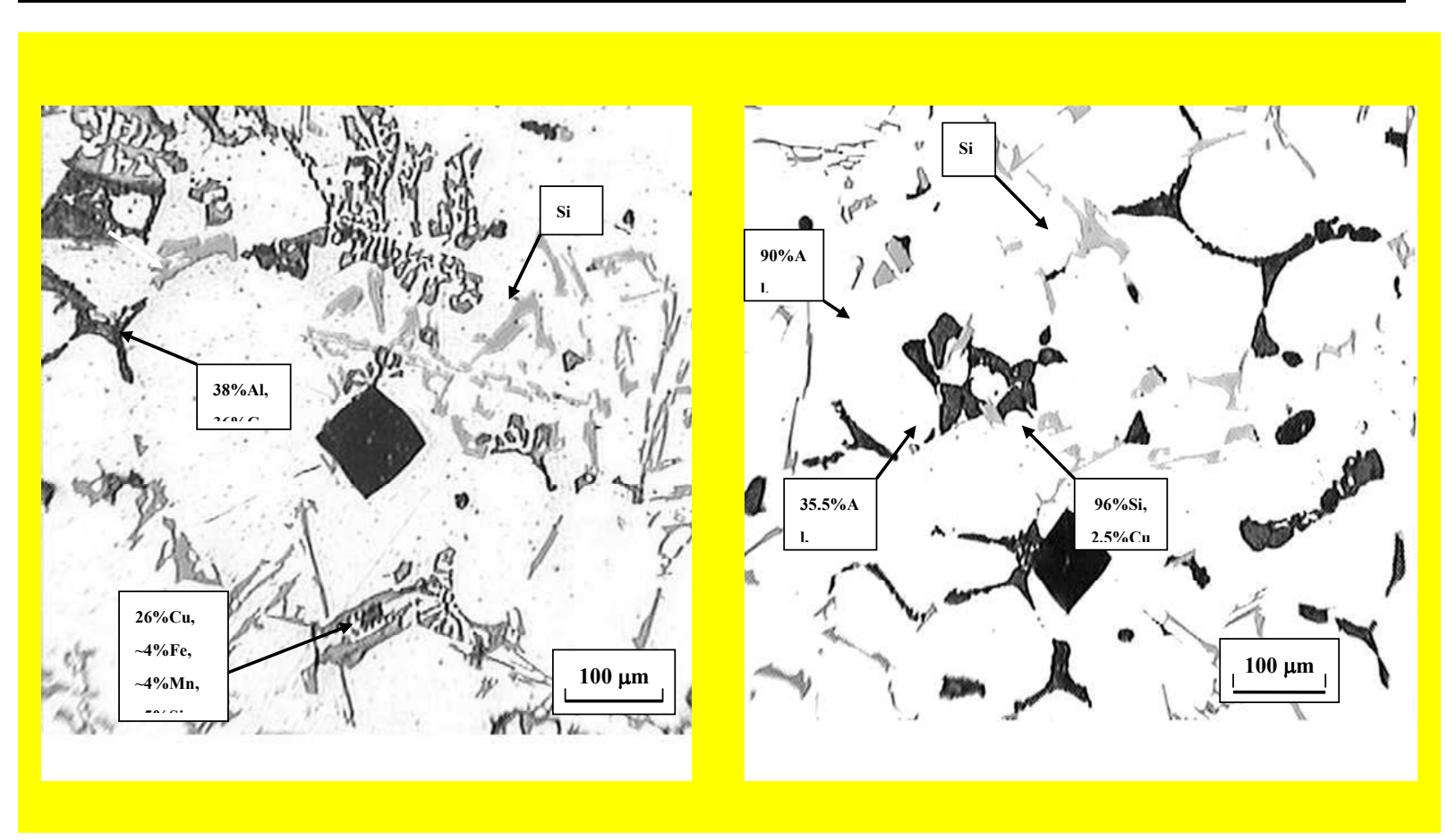

Figure 3.4-3. SEM images and X-ray spectra semi-quantitative analyses of constituent particles in Al-319 automotive castings 


\section{Section 3: Databases}

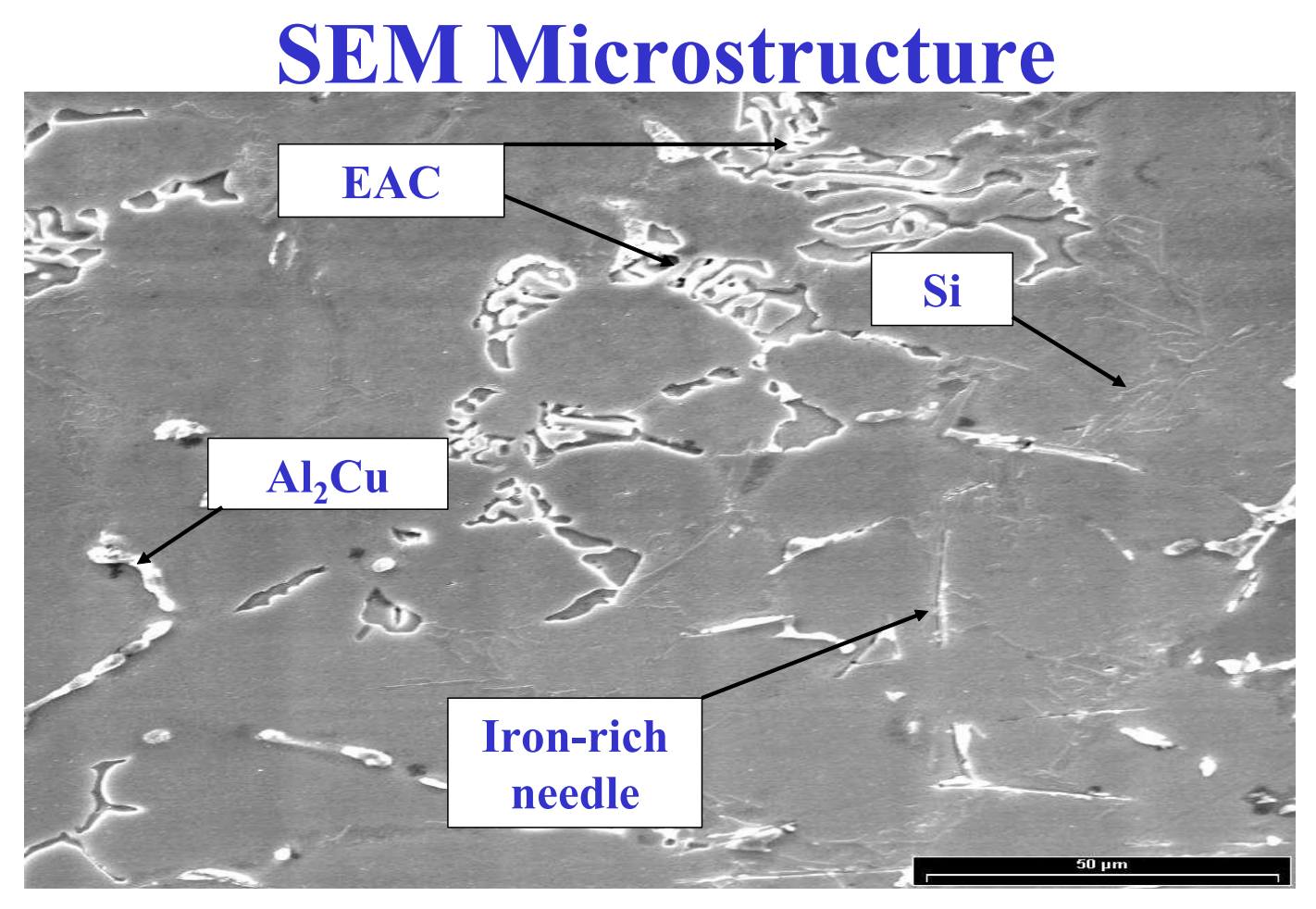

Figure 3.4-4. SEM image of metallographic section cut from end-chilled plate casting. Phases containing low atomic weight elements ( $\mathrm{Al}, \mathrm{Si}, \mathrm{Mg}$ ) appear dark and phases containing heavy elements $(\mathrm{Cu}, \mathrm{Fe})$ appear bright. Iron-rich needles are $\mathrm{Al}_{5} \mathrm{FeSi}$. Interdendritic $\theta$-phase $\left(\mathrm{CuAl}_{2}\right)$ and silicon are evident 


\section{Section 3: Databases}

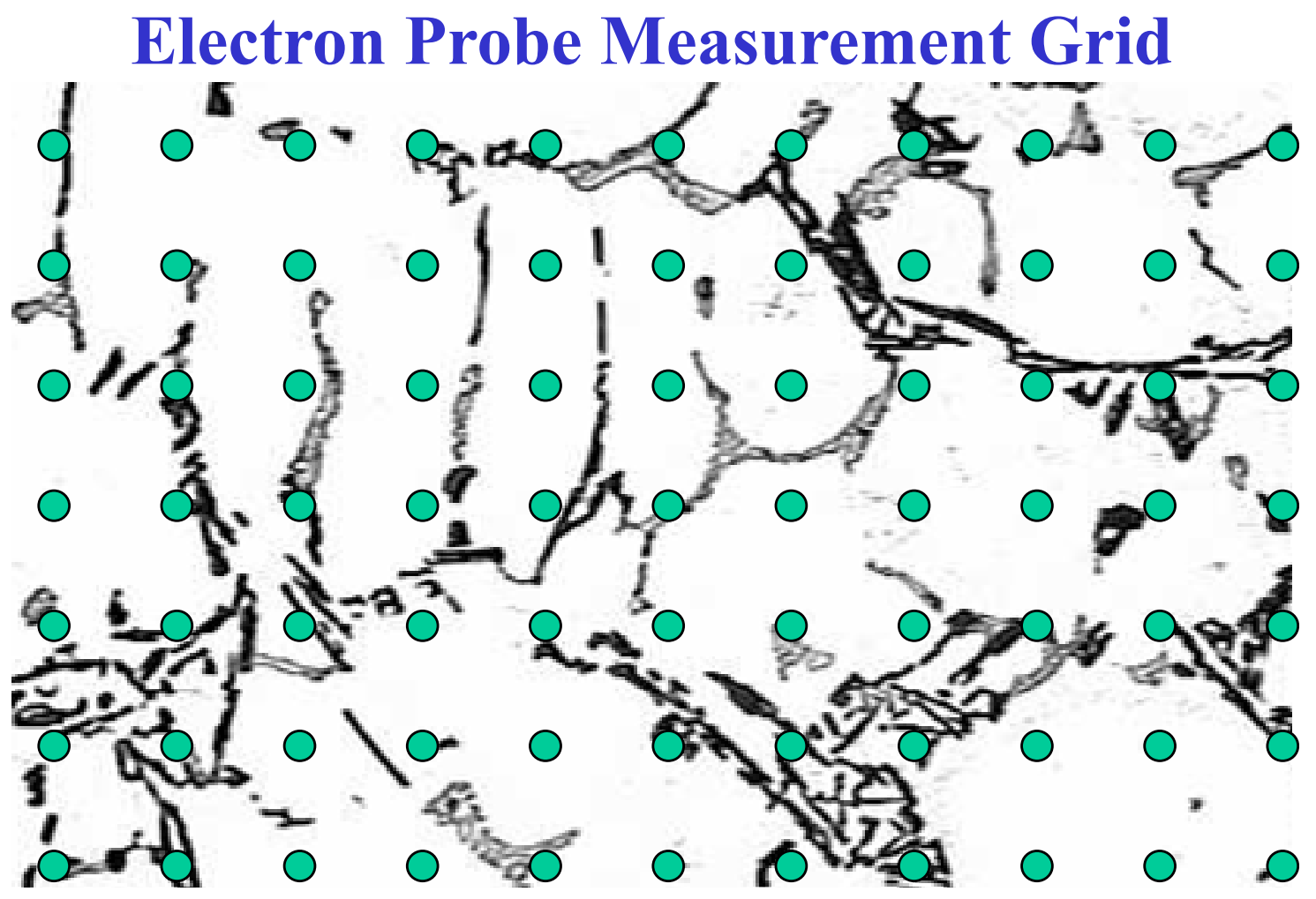

Figure 3.4-5. Illustration of grid of measurement points used for electron beam microprobe analysis of solute distribution averaged over a representative number of dendrite arms 


\section{Section 3: Databases}

\section{Micronrobe Data}

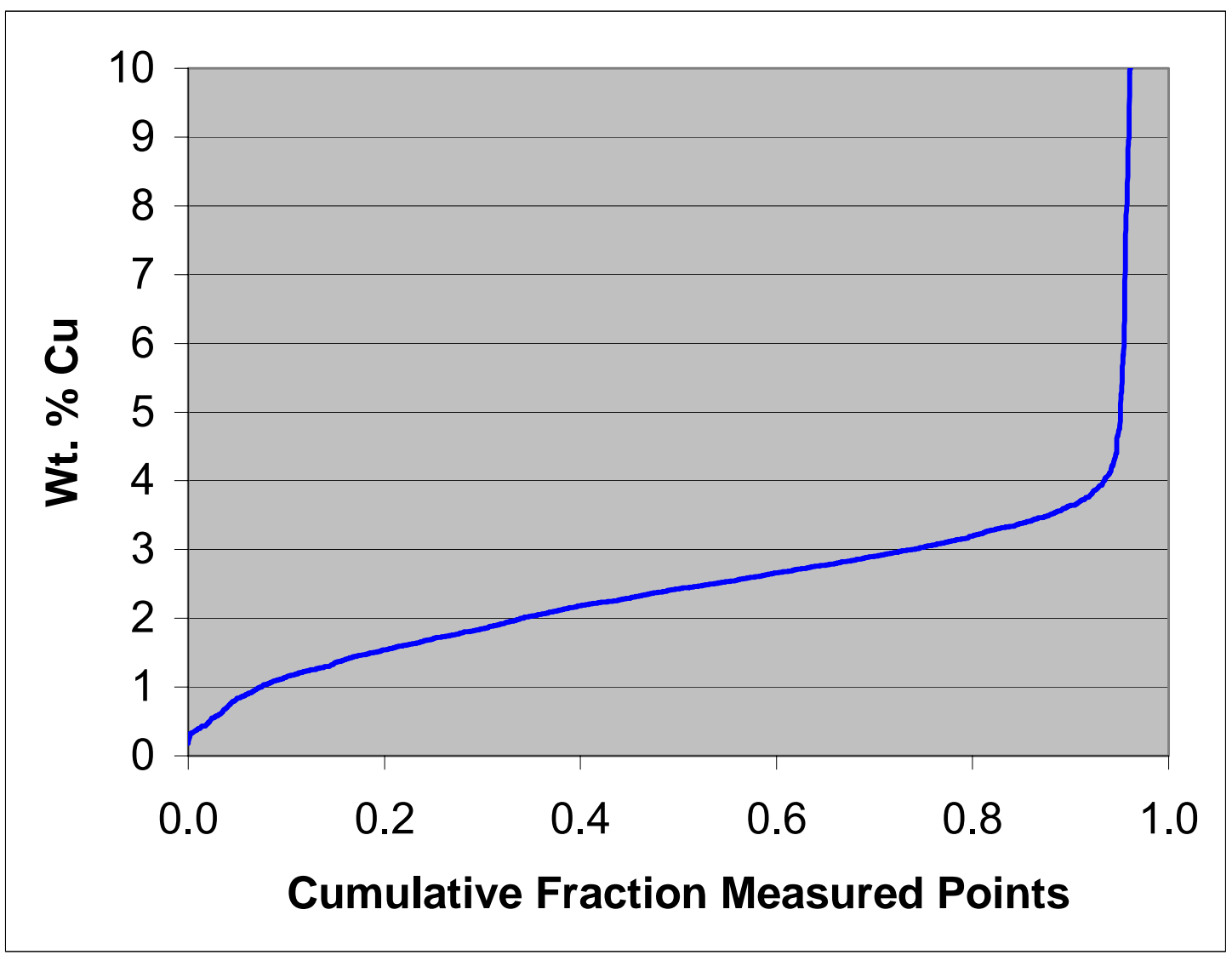

Figure 3.4-6. Example of averaged copper distribution across a characteristic dendrite volume element obtained by electron beam microprobe analysis 


\section{Section 3: Databases}

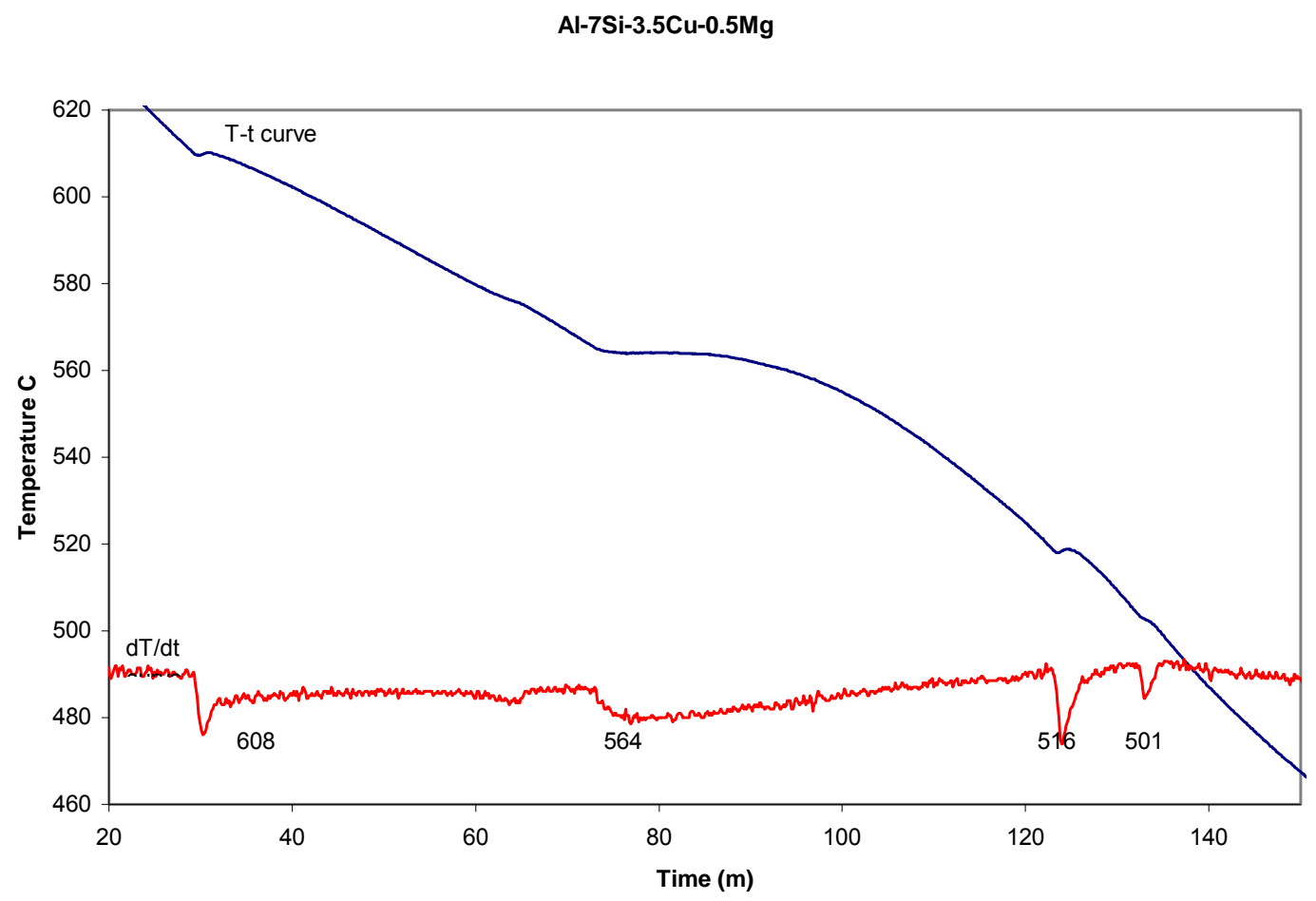

Figure 3.4-7. Cooling curve for an $\mathrm{Al}-7 \% \mathrm{Si}-3.5 \% \mathrm{Cu}-0.5 \% \mathrm{Mg}$ quaternary alloy and (at bottom) the time derivative of the cooling curve. Thermal arrests and/or recalescence on the cooling curve and peaks/dips in the derivative curve mark temperatures for the initiation of phase formation 


\section{Section 3: Databases}

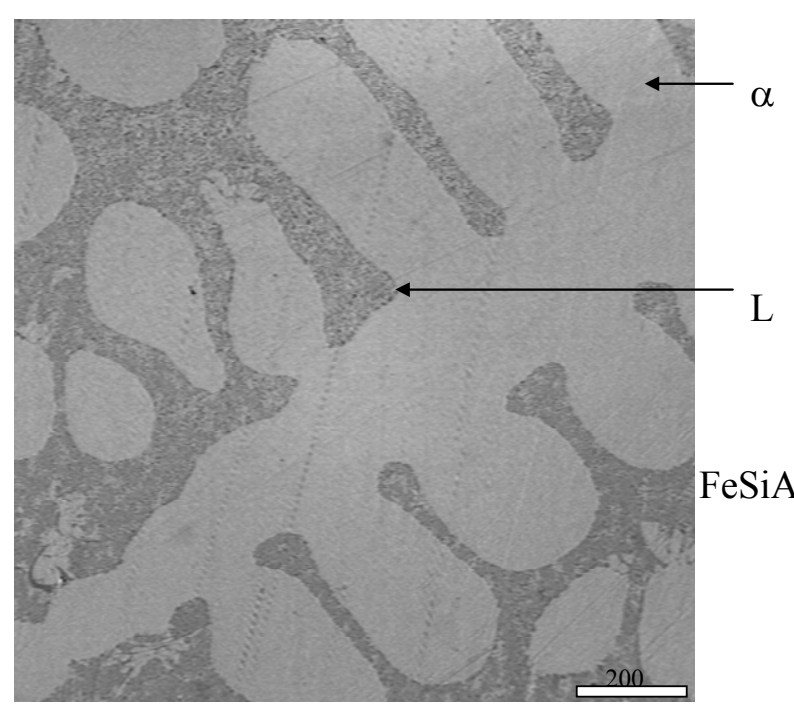

$571^{\circ} \mathrm{C}$

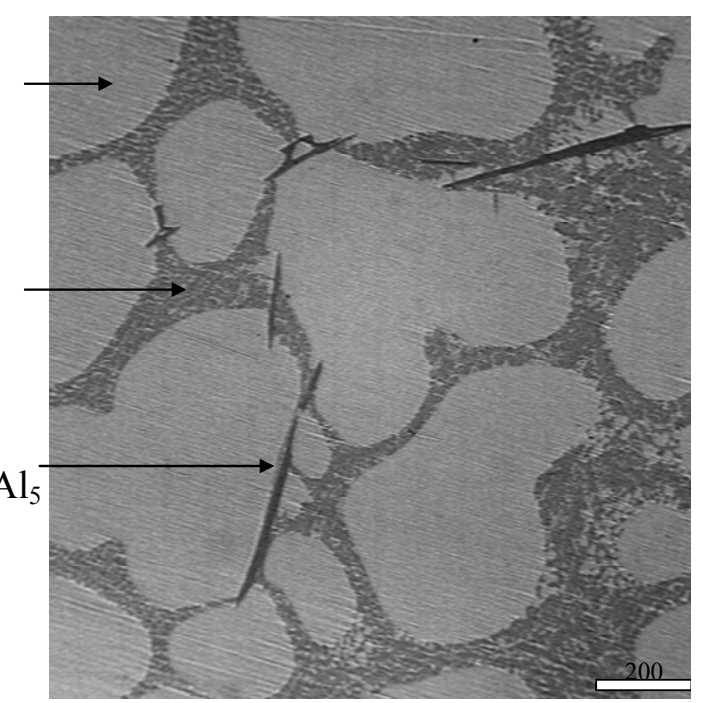

$564^{\circ} \mathrm{C}$

Al-7\%Si-3.5\%Cu-0.5\%Mg-0.5Fe allov

Figure 3.4-8a. Interrupted solidification samples. Left: Sample quenched at $571^{\circ} \mathrm{C}$ shows formation of $\alpha$-phase dendrites. Right: Sample quenched at $564^{\circ} \mathrm{C}$ shows continued formation of $\alpha$-phase dendrites and initiation of iron-rich intermetallic, $\mathrm{Al}_{5} \mathrm{SiFe}$ 


\section{Section 3: Databases}

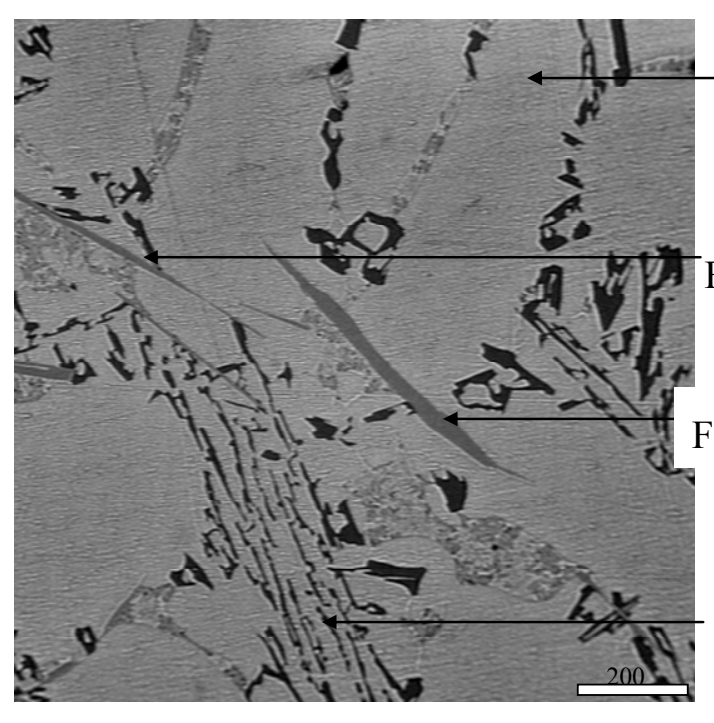

$561^{\circ} \mathrm{C}$

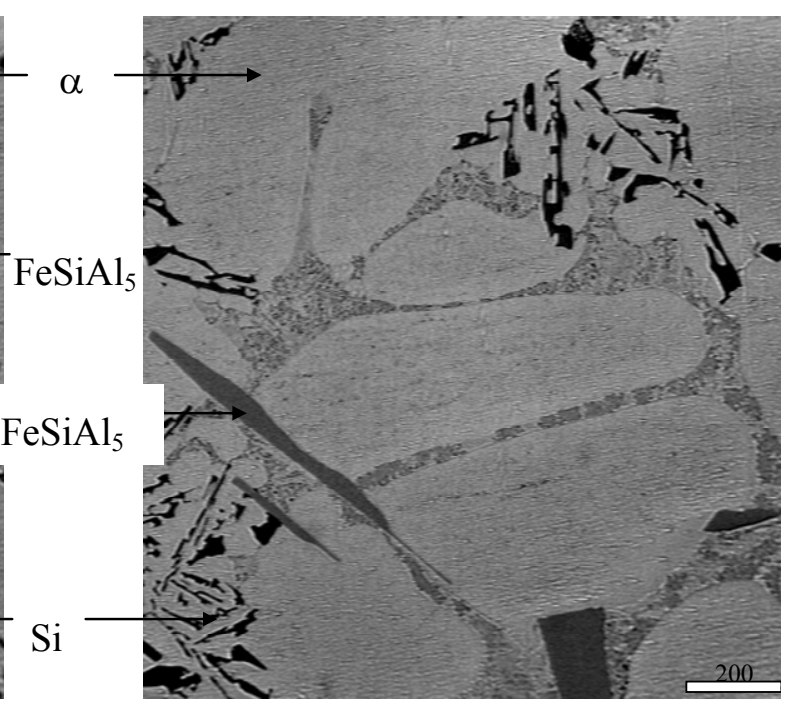

$555^{\circ} \mathrm{C}$

\section{Al-7\% $\%$ Si-3.5\%Cu- $0.5 \% \mathrm{Mg}-0.5 \mathrm{Fe}$ allov}

Figure 3.4-8b. Interrupted solidification samples. Left: Sample quenched at $571{ }^{\circ} \mathrm{C}$ shows formation of $\alpha$-phase dendrites. Right: Sample quenched at $564^{\circ} \mathrm{C}$ shows continued formation of $\alpha$-phase dendrites and initiation of iron-rich intermetallic, $\mathrm{Al}_{5} \mathrm{SiFe}$ 


\section{Section 3: Databases}

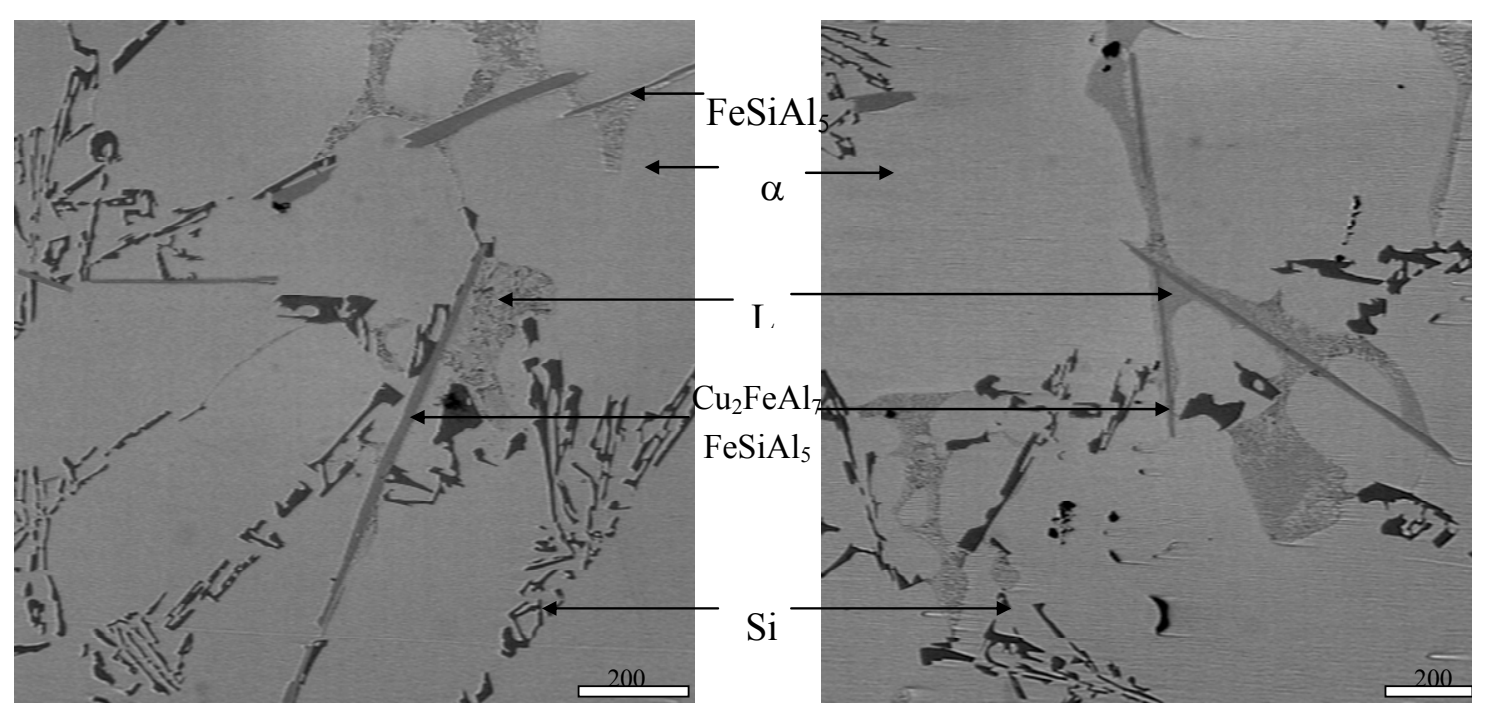

$545^{\circ} \mathrm{C}$

Al-7\% $\%$ Si-3.5\%Cu- $0.5 \% \mathrm{Mg}-0.5 \mathrm{Fe}$ allov

Figure 3.4-8c. Interrupted solidification samples. Left: Sample quenched at $545^{\circ} \mathrm{C}$ shows continued formation of $\alpha$-dendrites and $\mathrm{Al}_{5} \mathrm{FeSi}$ platelets and initiation of $\alpha+$ silicon colonies. Some $\mathrm{Al}_{5} \mathrm{FeSi}$ platelets are converting to $\mathrm{Cu}_{2} \mathrm{FeAl}_{7}$. Right: Same 


\section{Section 3: Databases}

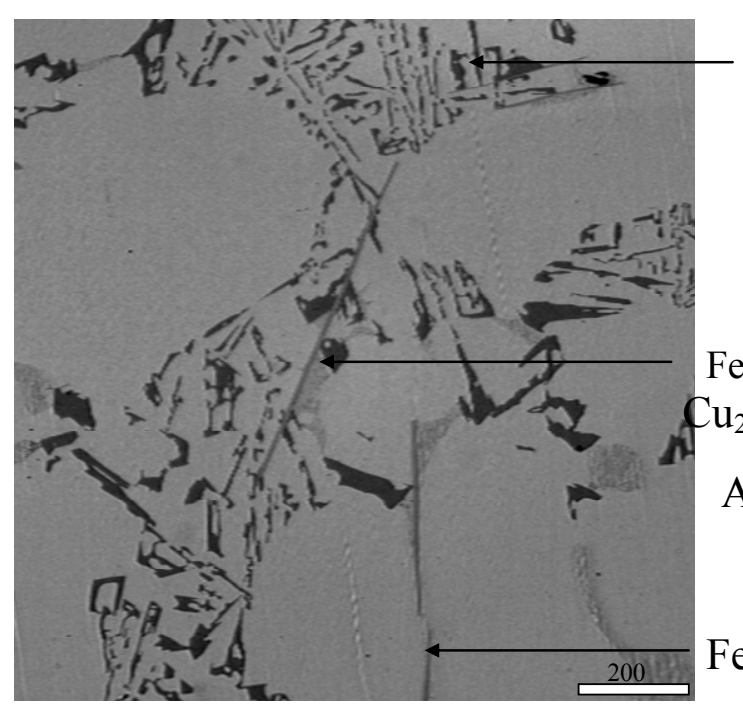

$520^{\circ} \mathrm{C}$

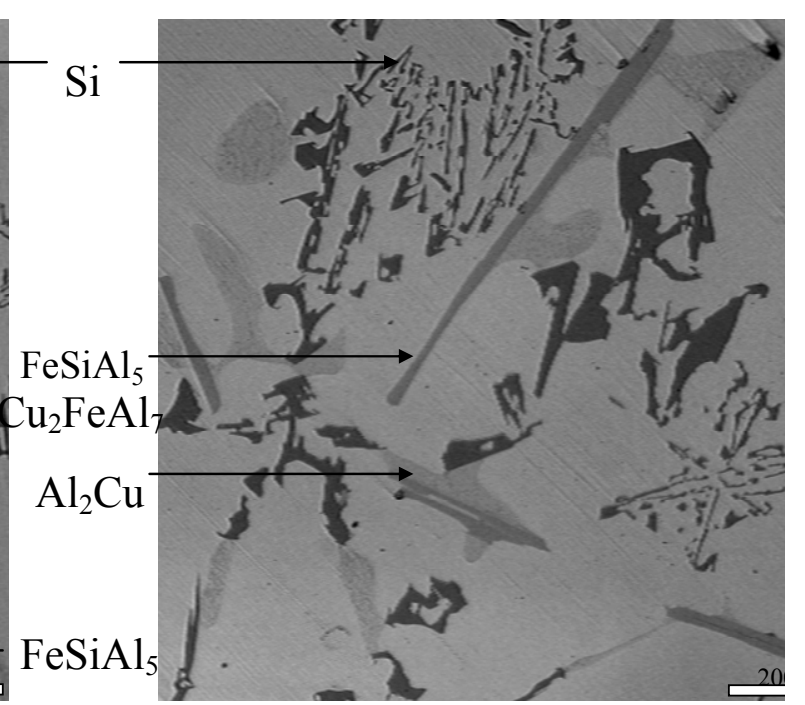

$510^{\circ} \mathrm{C}$

\section{Al-7\% $\%$ i- $3.5 \% \mathrm{Cu}-0.5 \% \mathrm{Mg}-0.5 \mathrm{Fe}$ allov}

Figure 3.4-8d. Interrupted solidification samples. Left: Sample quenched at $520^{\circ} \mathrm{C}$ shows continued formation of $\alpha$-dendrites, $\mathrm{Al}_{5} \mathrm{FeSi}$ platelets, $\alpha+$ silicon colonies, and conversion of $\mathrm{Al}_{5} \mathrm{FeSi}$ to $\mathrm{Cu}_{2} \mathrm{FeAl}_{7}$. Right: Sample quenched at $510^{\circ} \mathrm{C}$. $\mathrm{Al}_{2} \mathrm{Cu}, \theta$-phase, has formed at quaternary eutectic

\subsection{Diffusivity Database}

A diffusivity database has been developed for Al-Cu-Si-Mg alloys. Values from the literature have been compared with data in public software databases and with our measurements. Where there was substantial disagreement in values, we repeated our measurements and weighed the experimental results most heavily.

Experimental values were obtained from both single-phase and two-phase diffusion couples. The ends of the couples are solution treated individually to remove coring and nonequilibrium constituents. Then the surfaces of the couples to be mated are cleaned, polished, pressed together, and sealed in argon. The couples are heat-treated, quenched, sectioned longitudinally, polished, and then concentration profiles are measured by electron probe microanalysis, from 


\section{Section 3: Databases}

which diffusion coefficients are determined. The single-phase couples give diffusion coefficients in the primary $\alpha$-phase, while the two-phase couples indicate the role that $\mathrm{Si}$ particles can have in providing a high diffusivity path for copper and silicon diffusion.

Because of the limited solubility of $\mathrm{Cu}$ and $\mathrm{Si}$ in $\mathrm{Al}$, the effect of concentration on $D_{\mathrm{CuCu}}^{\alpha}$ and $D_{S i S i}^{\alpha}$ was found to be negligible. As a result, data from ternary diffusion couples can be evaluated using the Grube analysis, which can only be used when diffusion coefficients are constant. In addition, the cross coefficients, $D_{C u S i}^{\alpha}$ and $D_{S i C u}^{\alpha}$, were found to be negligible.

Data are summarized in Tables 3.5-1 and 3.5-2. Here it can be seen that diffusion coefficients of copper are insensitive to variations in $\mathrm{Cu}$ and $\mathrm{Si}$ concentration, but are changed as much as $50 \%$ by the addition of $0.45 \% \mathrm{Mg}$. The same can be said about the Si diffusion coefficients, although the effect of $\mathrm{Mg}$ is only half as great as for $\mathrm{Cu}$.

With regard to the diffusion of copper in two-phase alloys, a comparison of measured and tracer values is shown in Table 3.5-3. A four to five fold increase is seen at typical solution heat treatment temperatures for aluminum casting alloys. The result is that two-phase regions act as a short circuit path for redistributing $\mathrm{Cu}$ during solutionizing treatments.

Table 3.5-1. Diffusion Coefficients of Copper in Al-Si-Cu-Mg Alloys

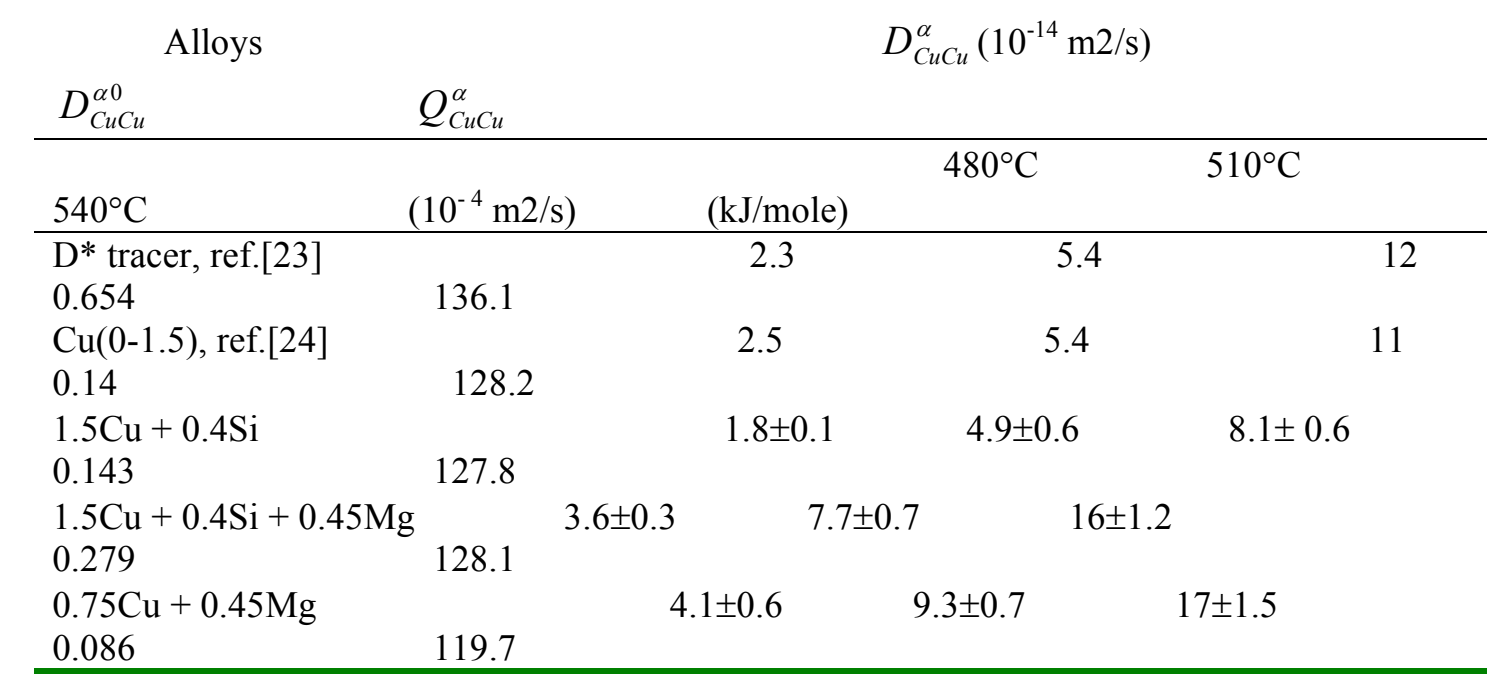




\section{Section 3: Databases}

\begin{tabular}{|c|c|c|c|c|c|c|}
\hline Alloys & & & & $D_{S i S}^{\alpha}$ & $\left(10^{-14} \mathrm{~m}^{2} / \mathrm{s}\right)$ & \\
\hline$D_{S i S i}^{\alpha 0}$ & $Q_{S i S i}^{\alpha}$ & & & & & \\
\hline $540^{\circ} \mathrm{C}$ & $\left(10^{-4} \mathrm{~m}^{2} / \mathrm{s}\right)$ & & $(\mathrm{kJ} /$ mole $)$ & $480^{\circ} \mathrm{C}$ & $510^{\circ} \mathrm{C}$ & \\
\hline $\begin{array}{l}\text { D* tracer, ref.[25] } \\
0.35\end{array}$ & 123.9 & & 8.8 & 19 & & 38 \\
\hline $\begin{array}{l}\mathrm{Si}(0-0.4), \text { ref.[26] } \\
0.34\end{array}$ & 124.2 & & 8.4 & 18 & & 36 \\
\hline $\begin{array}{l}1.5 \mathrm{Cu}+0.4 \mathrm{Si} \\
0.159\end{array}$ & 119.6 & & $7.7 \pm 0.8$ & $18 \pm 1.5$ & $32 \pm 5$ & \\
\hline $\begin{array}{l}1.5 \mathrm{Cu}+0.4 \mathrm{Si}+0.45 \mathrm{Mg} \\
0.086\end{array}$ & $\begin{array}{l}\text { g } \\
113.0\end{array}$ & $12 \pm 0.6$ & $24 \pm 1.8$ & & $42 \pm 2.5$ & \\
\hline $\begin{array}{l}0.2 \mathrm{Si}+0.45 \mathrm{Mg} \\
0.081\end{array}$ & 113.7 & & $11 \pm 0.5$ & $19 \pm 1.3$ & $42 \pm 2$ & \\
\hline
\end{tabular}

Table 3.5-3. Diffusion Coefficients of Copper in Single Phase $\alpha-\mathrm{Al}$ and two phase $\alpha-\mathrm{Al}+13$ vol.\% Si alloys

\begin{tabular}{|c|c|c|c|c|c|c|}
\hline \multirow[b]{2}{*}{ Matrix } & \multicolumn{4}{|c|}{$\mathrm{D}_{\mathrm{CuCu}}\left(10^{-14} \mathrm{~m}^{2} / \mathrm{s}\right)$} & \multirow{2}{*}{$\begin{array}{c}\mathrm{D}^{0} \\
\left(10^{-4} \mathrm{~m}^{2} / \mathrm{s}\right)\end{array}$} & \multirow{2}{*}{$\begin{array}{c}\mathrm{Q} \\
(\mathrm{kJ} / \mathrm{mol})\end{array}$} \\
\hline & $450^{\circ} \mathrm{C}$ & $480^{\circ} \mathrm{C}$ & $510^{\circ} \mathrm{C}$ & $540^{\circ} \mathrm{C}$ & & \\
\hline Pure Al (Cu Tracer) & .95 & 2.34 & 5.39 & 11.67 & 0.654 & 136 \\
\hline Single phase $\alpha$ & - & 1.81 & 4.94 & 8.12 & 0.143 & 128 \\
\hline Two phase, $\alpha+\mathrm{Si}$ & 4.36 & 11.1 & 19.5 & - & 0.149 & 118 \\
\hline
\end{tabular}

\subsection{Tensile Properties Database}

The overall objective of this task is to obtain and validate a database to relate tensile properties for industrial multi-component alloys to casting and solution treatment process parameters, alloy composition, and microstructural features. The features include grain size, fraction and size of porosity, fraction of soluble and insoluble phase, globularization of insoluble phases, and 160 


\section{Section 3: Databases}

constituent particle size. The database has been developed by mining the existing literature and by selected experimentation aimed at producing a wide range of as-cast microstructures in a series of Al-Si-Cu-Mg (Al-319 family of alloys) and Al-Si-Mg (Al-356 family) casting alloys and varying casting and heat treatment process parameters and then measuring the influence on tensile properties of as-cast microstructure and heat treatment. The results are analyzed to obtain a quantitative design rule based on a minimum set of process and microstructure parameters.

\subsubsection{Approach}

End-chilled plate castings are used to prepare test coupons that experience a wide range of solidification conditions and exhibit a wide variety of as-cast microstructures and mechanical properties. Coupons cut from the cast plates are solution treated for times ranging from 0 to 32 hours, quenched, and given the same aging treatment. Microstructural parameters, including dendrite arm spacing, dendrite cell size, and volume percent porosity, are measured. Tensile properties are measured for the full range of solidification conditions and solutionizing times. A multi-dimensional least square analysis is used to develop an empirical relation, i.e. a design rule relating tensile properties and a minimum set of microstructure and processing parameters. The empirical relation is validated by casting, heat treating, and testing additional sets of test plates and comparing the second set of measured properties to the properties predicted by the relation determined from the first set of measurements.

We sectioned and measured several microstructural parameters of several automotive aluminum structural castings made in permanent molds, sand molds with and without copper chill blocks, and by the lost foam process. Dendrite spacing in the commercial automotive castings ranged from $20 \mu \mathrm{m}$ in regions adjacent to a large copper chill to $100 \mu \mathrm{m}$ in thick wall sand castings. Volume percent porosity ranged from less than $0.1 \%$ to $3 \%$. Generally, in regions of castings where high strength and toughness are specified the casting process is designed to produce fine dendrites and low porosity. 


\section{Section 3: Databases}

An end-chilled horizontal plate casting was designed to simulate a broad range of casting practice and to provide six standard round ( 0.505 " dia) tensile samples from each plate. A vertical plate casting was designed to simulate the solidification conditions in those regions where performance requirements are stringent. Two tensile specimens can be cut from each vertical plate. In order to simulate the as-cast microstructure adjacent to a copper chill block or in a thin walled permanent mold casting and to have enough thickness to provide standard 0.505 " tensile samples, three chills were placed around the end of the vertical plate casting. Volume percent porosity in well degassed melts and the size of the pores decrease in parallel with the dendrite spacing, as local solidification time is reduced. Dendrite spacing and volume \% porosity do not vary independently in these castings.

\subsubsection{Processing of End-Chilled Test Plate Castings}

Test plate castings are being cast from a series of alloys with slight variations from a nominal 319 composition of $\mathrm{Al}-7 \% \mathrm{Si}-3.5 \% \mathrm{Cu}-0.5 \% \mathrm{Fe}-0.33 \% \mathrm{Mg}$ (Note Table 3.6-1.) and a nominal 356 composition of $\mathrm{Al}-7 \% \mathrm{Si}-0.33 \% \mathrm{Mg}$. Iron is added to the $\mathrm{Al}-\mathrm{Si}-\mathrm{Cu}-\mathrm{Mg}$ alloy melts because it is omnipresent in automotive industry aluminum alloy castings. The test plates are chilled at one end. Plates cast from each series are cut into coupons for metallographic analysis and mechanical testing. Coupons are heat treated before testing. The data collected have been used to develop a process-property and a process-microstructure database.

The first set of test plate castings are $229 \mathrm{~mm}$ x $178 \mathrm{~mm}$ x $25 \mathrm{~mm}$ (9 in x 7 in x 1 in), are chilled at one end, and have a tapered cylindrical riser, 100mm high $\times 88 \mathrm{~mm}$ in diameter at its base and $100 \mathrm{~mm}$ in diameter at the top (4 in $x 3.5$ in $\phi$ to 4 in $\phi$ ). Note Figure 3.6-1 for placement of the chill and riser. The aim is to provide a range of thermal conditions, cooling rate and temperature gradient, and, thus, as-cast microstructures that span the range observed in commercial sand and permanent mold casting practice for automotive applications.

Seven molds per heat are cast for each alloy series. The molds in an alloy series are poured from a single $52 \mathrm{~kg}(125 \mathrm{lb}$.) heat with a controlled composition, melt treatment, and pouring practice. Heats are melted in a $3,000 \mathrm{~Hz}$ induction furnace in an alumina crucible. Because 


\section{Section 3: Databases}

the plate castings are poured from the same melt, the seven plates cast in one series are expected to have the same composition and initial melt hydrogen content.

Melts for the first seven alloy series were degassed by purging with a low dew point $\mathrm{Ar}-10 \% \mathrm{Cl}$ mixture. In later heats melts have been purged with ultra-high purity, low dew point Ar. Hydrogen content in the melt is evaluated by a reduced pressure $(>5 \mathrm{~mm})$ test.

Melt charges are made from $99.9 \% \mathrm{Al}, \mathrm{Al}-32 \% \mathrm{Si}$ or $\mathrm{Al}-50 \% \mathrm{Si}, \mathrm{Al}-50 \% \mathrm{Cu}, \mathrm{Al}-5 \% \mathrm{Fe}$ master alloys, and $\mathrm{Mg}$ rod. The $\mathrm{Al}-50 \% \mathrm{Cu}$ and $\mathrm{Al}-5 \% \mathrm{Fe}$ hardener alloy were melted and poured into cast iron ingot molds at UConn from high purity starting materials. The Si nuggets used to make the $\mathrm{Al}-32 \% \mathrm{Si}$ master alloy contained about $0.2 \% \mathrm{Fe}$ and about $0.1 \%$ other impurities. Further Fe was picked up from tools used to stir the melts at temperatures above the master alloy's liquidus temperature, $925-950^{\circ} \mathrm{C}\left(1700-1750^{\circ} \mathrm{F}\right)$. All charge materials, except $\mathrm{Mg}$, are preheated to at least $250^{\circ} \mathrm{C}$ prior to being added to the induction furnace. Al-5\%Ti-1\%B and Al-10\% Sr are used for grain refining and eutectic modification.

Flaskless molds are prepared using \#80 silica sand with an inorganic (sodium silicate) binder that is set by blowing with $\mathrm{CO}_{2}$. Molds are filled from an unpressurized gating system from the tilt induction furnace. Fill rate is controlled by placing a 15 pore per mm ceramic filter in the runner and by tilting the bottom boards for the molds upward at a 10-degree angle toward the chill end. Temperature of the melt in the induction furnace is maintained at 725 to $740^{\circ} \mathrm{C}\left(1340-1360^{\circ} \mathrm{F}\right)$ during tilt pouring. The plates are allowed to slow cool overnight in the mold prior to shakeout. The plates are cut transverse to the long axis into two sets of coupons, one set for tensile testing and a second set for metallographic analysis, as sketched in Figure 3.6-1. The coupons for tensile testing are approximately $25 \times 25 \times 178 \mathrm{~mm}$. The coupons for metallographic analysis are about one-half as wide.

Most coupons will be heat-treated before further machining and analysis. Solutionizing and aging are carried out in a forced air resistance heated furnace. Coupons are tied in groups by wire with a separation of at least $25 \mathrm{~mm}$ between coupons. At the end of the solutionizing period a bundle of samples are pulled from the furnace and quenched within a few seconds in warm water. All heat-treated coupons are quenched in warm water after solution treatment, 


\section{Section 3: Databases}

allowed to stand at room temperature for 24 hours before age hardening. Al-Si-Cu-Mg alloys are age hardened at $230^{\circ} \mathrm{C}$ for three and one-half hours. Al-Si-Mg alloys are age hardened at $165^{\circ} \mathrm{C}$ for five and one-half hours. Coupons from one plate are age hardened directly from the as-cast condition --- with no solution treatment. Coupons from another four plates are solution treated at 2, 4, 8, and 32 hours. Coupons from the remaining two plates per alloy series have been given two-stage solution treatments or solution treatments less than 2 hours.

Tensile test coupons are machined to standard 0.5 in diameter $\mathrm{x} 2$ in gauge length round bars with threaded ends. Coupons for tensile testing are shipped to Westmoreland Mechanical Testing and Research, Inc. for machining and testing. Tensile bars are pulled to failure with an extensometer attached. If the measured elastic modulus deviates by more than $10 \%$ from the handbook value, the tensile data are not reported. Ultimate tensile strength, $0.1 \%$ or $0.2 \%$ offset yield strength, $\%$ elastic and \% plastic elongation before failure are recorded.

Microstructure in as-cast and heat treated samples are examined by optical microscopy, quantitative microscopy, and scanning electron microscopy. Limited transmission electron microscopy has been done to date.

Dendrite arm spacing and dendrite cell spacing in the as-cast alloys are measured on the coupons prepared for microstructural analysis. Dendrite cell spacing is measured by inscribing random lines over the microstructure and measuring the average distance between intersections of the lines with interdendritic regions. Alternatively, dendrite arm spacing is measured by identifying primary arms with several perpendicular secondary arms in the plane of view and measuring the average perpendicular distance between secondary arms. Volume percent porosity is measured by comparing the density of sections cut from the metallography coupons of the end-chilled cast plate to the density of a control sample of the same composition (barring macrosegregation along the length of the plates). The control sample for each composition is made by remelting and then chill casting a small piece of the cast plate.

For some of the the Al-Si-Mg alloys vertical plate $(150 \times 100 \times 25 \mathrm{~mm})$ molds were prepared, four plates per mold, with one or three copper chill blocks placed vertically in the mold against one end $(150 \times 25 \mathrm{~mm})$ of the plate. A central, tapered cylindrical riser is used to feed the four 


\section{Section 3: Databases}

plates. Two molds, eight plates, are poured from forty pound heats melted in a propane/forced air fired crucible furnace. Two tensile coupons and two metallography coupons are cut from each plate. The coupons are processed and tested by the same procedures as for coupons cut from the horizontal end-chilled plate castings.

\subsubsection{Process-Microstructure-Property Relations for Al-Si-Cu-Mg Alloys}

3.6.2.1 Tensile Test Results for Al-Si-Cu-Mg Alloy: Nominal Composition The tensile properties of the nominal alloy, Al-7\% $\mathrm{Si}-3.5 \% \mathrm{Cu}-0.33 \% \mathrm{Mg}-0.5 \% \mathrm{Fe}$, are plotted simultaneously against dendrite arm spacing and solution treatment time at $505^{\circ} \mathrm{C}$ in Figure 3.6-2 The variation in dendrite arm spacing and volume percent porosity with distance from the chilled end of the horizontal plate castings is plotted for the nominal Al-Si-Cu-Mg alloy in Figure 3.6-3.

The contour plots in Figure 3.6-2 illustrate a significant increase in tensile strength for fine dendrite arm spacing and extended solution treatment time. The lowest values in each contour plot represent samples that were aged in the as-cast condition (without prior solution treatment). The remainder of the contour surface demonstrates that tensile strength increases continuously as dendrite arm spacing and volume percent porosity decrease and solution treatment time at $505^{\circ} \mathrm{C}$ increases. The contour plots illustrate a two-fold (100\%) increase in tensile strength from coarse dendrites and two-hour solution treatment to fine dendrites and 32-hour solution treatment. Sharply lower values are found for samples that were aged in the as-cast (slow cooled) condition. Considering samples that were aged without prior solution treatment, the overall increase in tensile strength is three-fold (200\%). Qualitatively similar results found for other Al-Si-Cu-Mg compositions and conditions studied are presented in a later subsection.

The yield strength increases significantly for the first increment of solution treatment and then reaches a plateau. The decrease of yield strength with increasing dendrite arm spacing and volume percent porosity is not as dramatic as the variation of tensile strength. Significant increase in yield strength occurs between samples that are aged without solutionizing and those that are given a two-hour solution treatment. The increase of yield strength from two-hour 


\section{Section 3: Databases}

solution treatment and coarse dendrites to fine dendrites and 32-hour solution treatment is less than $20 \%$.

Elongation properties peak at fine dendrite arm spacing and long solution treatment times. Increasing solution treatment time at $505^{\circ} \mathrm{C}$ has a significant effect on ductility (\% plastic eleongation at fracture) for locations with fine dendrite arm spacing. Samples from locations with coarse microstructures and long local solidification times have very low ductility. Ductility is not improved significantly by extended solution heat treatment.

Typical results for dendrite arm spacing are plotted in Figure 3.6-3. Dendrite arm spacing increases continuously with distance from the chilled end of the plate. Sections close to the chill end have a dendrite spacing and, thus, a local solidification time comparable to permanent mold castings. Sections of the plate further from the end-chill have dendrite arm spacings and local solidification times comparable to sand castings.

Typical results for volume percent porosity are plotted as a function of distance from the chill in Figure 3.6-3. Both the size and volume fraction of pores increase continuously with distance from the chill, as does dendrite arm spacing. Dendrite arm spacing and volume percent porosity do not change independently in the end-chilled plate castings.

\subsubsection{Analysis of Tensile Strength Results for Al-Si-Cu-Mg Alloy: Nominal Composition} Our starting hypothesis is that for a fixed alloy composition, pouring practice, quenching practice and aging treatment, the primary factors that influence the local tensile properties of the cast and heat treated alloy are the local thermal parameters during solidification (local solidification time and temperature gradient), the amount of dissolved hydrogen in the melt as the alloy enters the mold, and the solution treatment thermal cycle. The local solidification time determines the scale of the as-cast microstructure: dendrite arm spacing, silicon-phase particle size, copper-rich and iron-rich intermetallic particle size, volume percent porosity, and size of porosity. Dendrite arm spacing is selected here to represent local solidification time and the size scale of the as-cast microstructure. 


\section{Section 3: Databases}

Volume percent porosity in the as-cast microstructure is a direct result of dissolved hydrogen, local solidification time, and temperature gradient and is a predominant factor in limiting alloy ductility. Volume percent porosity in the as-cast microstructure can be determined by quantitative metallography or by density measurement.

The solution treatment thermal cycle influences the effectiveness of the dissolution of nonequilibrium phases, the formation of equilibrium phases, and the distribution of strengthening elements through the terminal solid solution matrix ( $\alpha$-aluminum dendrite cores). Time at the solution treatment temperature is the single parameter used to represent the solution treatment process.

A multidimensional least square procedure is used to express the tensile strength as a linear function of dendrite arm spacing (DAS), volume fraction porosity $(\% \mathrm{P})$, and solution treatment time $\left(\theta_{\mathrm{S}}\right)$ :

$$
U T S=a(D A S)+b(1+\% P)+c\left(\theta_{S}\right)+d
$$

where $\mathbf{a}, \mathbf{b}, \mathbf{c}$, and $\mathbf{d}$ are parameters to be determined by least square analysis. The least square equation determined for the nominal alloy $(\mathbf{a}=-2.3, \mathbf{b}=-33.2, \mathbf{c}=+14.8$, and $\mathbf{d}=+354.7 \mathrm{MPa})$ is shown in Figures 3.6-4. The result is used to plot curves of tensile strength versus solutionizing time for different dendrite arm spacings. The points plotted in Figure 3.6-4 are the measured tensile data. The computed tensile strength is compared to measured tensile strength in Figure 3.6-5 and the 45-degree line shows excellent agreement, with an $\mathrm{R}^{2}$ value of 0.97 .

The comparisons in Figures 3.6-4 and 3.6-5 are between the predictions of the least square relation and the data that were used to develop it. To validate the empirical relation tensile properties were measured for another set of plates of the nominal composition that were cast and heat treated according to the experimental procedures described above. Tensile properties measured on the second set of plate castings and the predicted properties based on the least square fit to the original data set are compared in Figure 3.6-6. Again the comparison fits a line 


\section{Section 3: Databases}

of slope equal to one (45-degrees) and now the $\mathrm{R}^{2}$ value is 0.95 , not quite as strong a correlation as when comparison is made to the original data set.

The parameters used to represent as-cast microstructure, dendrite arm spacing and volume percent porosity, do not vary independently for our standard practice, note Figure 3.6-3. During the course of the program plates have been cast where the dissolved hydrogen exceeded our standard practice. In these plates, different values for volume percent porosity are obtained for the same dendrite arm spacing. In Figure 3.6-7 the least square fit is used to predict iso-strength lines on a plot of volume percent porosity versus dendrite arm spacing. Then the measured tensile strength is marked over the points that indicate the combination of porosity and dendrite arm spacing for the location tested. The correlation of predicted values and measured values is surprisingly good. This gives us confidence that equation 3.6-1 with validated parameters for $\mathbf{a}, \mathbf{b}, \mathbf{c}$, and $\mathbf{d}$ can be used as a design rule to predict the properties of Al-Si-Cu-Mg casting alloys.

3.6.2.3 Tensile Test Results for Al-Si-Cu-Mg Alloy: Additional Compositions The results of tensile testing on Al-Si-Cu-Mg bars machined from cast, solution treated, and age hardened coupons are presented in Figures 3.6-8 to 3.6-13. The ultimate tensile strength and the \% plastic strain before failure are plotted against distance from the chill and solution treatment time at $505^{\circ} \mathrm{C}$. Note from Figure 3.6-3 that both dendrite arm spacing and volume percent porosity increase for coupons cut at greater distance from the chill. Only one tensile bar was pulled for each combination of distance from the chill and solution treatment time. Roughness in the plots, presumably, would be reduced if several samples were tested and the results averaged for each combination.

The three-dimensional plots in Figure 3.6-8 and 3.6-9 compare the tensile strength (UTS) and percent permanent deformation before fracture (\% $\left.\mathrm{el}_{\text {plastic }}\right)$ measured at three levels of copper content. Tensile strength increases moderately as copper content is increased. Ductility for this family of alloys is extremely low. Adding $\mathrm{Cu}$ to increase strength does not appear to 


\section{Section 3: Databases}

present a major penalty in ductility at fine dendrite spacing and long solution treatment times, where $\%$ elongation to failure is greater.

Figures 3.6-10 and 3.6-11 compare the properties measured at three levels of Si content. The results do not indicate a reason to adjust Si content away from the 7\% (approx.) nominal level.

Figures 3.6-12 and 3.6-13 compare the properties measured for the nominal composition with those for low $\mathrm{Fe}$ and no $\mathrm{Mg}$. Strength is not changed significantly by changing from high to moderately low iron content. Ductility actually decreases, in these results, for the lower iron content. Removing Mg decreases strength by about 10,000 psi. Ductility increases when $\mathrm{Mg}$ is removed. $\mathrm{Mg}$ additions seem to be the most significant factor in improving strength.

In general the best combination of strength and ductility are observed, as expected, at the positions closest to the chill (fine dendrite spacing, small pore size, low volume percent porosity, small as-cast constituent size) and after extended solution treatment.

The strength increases sharply from the as-cast (slow cooled) and then aged condition to the 2-hour solution treatment and aged condition. Strength increases only slowly for longer solution treatment times. Strength and ductility are stronger functions of dendrite spacing than of solution treatment time. The big decrease in strength for coupons that were aged without solution treatment results, in large part, from slow cooling of the cast plates in the sand molds. Just a short solution treatment reverses the solute redistribution that occurred during slow cooling.

3.6.2.4 Summary for Al-Si-Cu-Mg Alloys For a family of alloys around $\mathrm{Al}-7 \% \mathrm{Si}-3.5 \% \mathrm{Cu}-0.33 \% \mathrm{Mg}-0.5 \mathrm{Fe}$ tensile strength increases for extended solution treatment and by achieving fine as-cast microstructures. Yield strength increases rapidly for short solution treatment times and plateaus. Ductility increases significantly for extended solution treatment for locations with fine as-cast microstructure and not at all for locations with coarse dendrite arm spacing.

An equation developed to fit the measured ultimate tensile strength to just three parameters, dendrite arm spacing, volume percent porosity, and solution treatment time, represents the measured data very well. When the empirical relation fit to the data is used to predict the 


\section{Section 3: Databases}

tensile strength of plates cast with the same composition but a wider variety of as-cast microstructures, the predictive capability is very good.

Similarly good fits and predictive capability are found for other compositions close to the nominal alloy composition. It is suggested that this approach to developing design rules for predicting tensile properties based on a limited set of process or microstructural parameters will be effective for other aluminum casting alloys and for the casting practices of other foundries, once calibrated for local conditions. A series of measurements, such as the ones described above, can be used to calibrate the design rule for local practices.

\section{$\underline{\text { 3.6.3 Process-Microstructure-Property Relations for Al-Si-Mg Alloys }}$}

For the Al-Si-Mg alloys cast in end chilled vertical plates the measured dendrite arm spacing increases from $<25 \mu \mathrm{m}$ at $13 \mathrm{~mm}$ from the end chill to $>50 \mu \mathrm{m} 100 \mathrm{~mm}$ from a single end chill. For plates cast with three chills dendrite arm spacing increases from $<20 \mathrm{~mm}$ near the chill The volume percent of porosity measured over the same range increased from $<0.1 \%$ near the chill to $<0.4 \%$ away from the chill.

The tensile properties of the nominal Al-Si-Mg alloy (grain-refined and not modified) are plotted versus solution treatment time at $540^{\circ} \mathrm{C}$ in Figure 3.6-14. The open symbols represent measured tensile strengths and the closed symbols represent measured $(0.2 \%$ offset $)$ yield strengths. The squares, diamonds, triangles, and circles represent samples with dendrite arm spacing of $17,35,44$, and $61 \mu \mathrm{m}$, respectively. No samples were aged without solution treatment. The minimum solution treatment time, $1 / 4$ hour, was used to reverse the effect of slow cooling in sand molds.

- Tensile strengths for coupons given extended solution treatments are about $20 \%$ greater than those given brief, $1 / 4$ hour solution treatments.

- Yield strength increases for the first couple of hours of solution treatment time and reaches a plateau. 


\section{Section 3: Databases}

- Elongations increase substantially even for samples with relatively coarse $(>60 \mu \mathrm{m})$ dendrite arm spacing.

Figure 3.6-15 compares the values of the tensile strength computed with equation (3.6-1), the proposed design rule, to the measured values for the nominal Al-Si-Mg alloys (grain-refined and not modified) cast in vertical end chill plate molds. The comparison, again, produces a 45 degree line but the $\mathrm{R}^{2}$ value is only 0.9. Slightly different equations and similar fits are obtained for variations about the nominal composition and for combinations of grain refinement and modification. The fits obtained for Al-Si-Cu-Mg alloys cast in the horizontal plate molds is consistently superior to the fits for the Al-Si-Cu alloys cast in the vertical plate molds.

In addition to the change in alloy family (from low ductility to moderate ductility), the mold configuration was changed from horizontal to vertical, data were collected over a smaller range of process variables, fewer data points were collected per condition, and the aging temperature was decreased from $230^{\circ} \mathrm{C}$ to $165^{\circ} \mathrm{C}$. Experiments where we change one variable at a time may clarify the reason(s) for the poorer predictability of tensile properties for Al-Si-Mg alloys. 


\section{Section 3: Databases}

Table 3.6-1. Aim Compositions and Melt Treatments for Test Plate Castings

(Aim concentrations for an element that differ from the nominal are shown in bold)

\begin{tabular}{|c|c|ccccc|}
\hline \multirow{2}{*}{$\begin{array}{c}\text { Melt } \\
\text { Treatment }\end{array}$} & Series & \multicolumn{5}{|c|}{ Aim Composition (wt\%) } \\
& No. & Si & Cu & Fe & Mg & Al \\
Not & $\mathbf{1}$ & 7.0 & 3.5 & 0.5 & 0.33 & Bal. \\
Grain & $\mathbf{2}$ & 7.0 & $\mathbf{2 . 5}$ & 0.5 & 0.33 & Bal. \\
Refined & $\mathbf{3}$ & 7.0 & $\mathbf{4 . 5}$ & 0.5 & 0.33 & Bal. \\
\& & $\mathbf{4}$ & $\mathbf{6 . 0}$ & 3.5 & 0.5 & 0.33 & Bal. \\
Unmodified & $\mathbf{5}$ & $\mathbf{8 . 0}$ & 3.5 & 0.5 & 0.33 & Bal. \\
& $\mathbf{6}$ & 7.0 & 3.5 & $\mathbf{0 . 0}$ & 0.33 & Bal. \\
\hline Grain & $\mathbf{7}$ & 7.0 & 3.5 & 0.5 & $\mathbf{0 . 0}$ & Bal. \\
Refined \& & $\mathbf{8}$ & 7.0 & 3.5 & 0.5 & 0.33 & Bal. \\
Unmodified & $\mathbf{9}$ & 7.0 & $\mathbf{2 . 5}$ & 0.5 & 0.33 & Bal. \\
\hline Not Grain & $\mathbf{1 0}$ & 7.0 & $\mathbf{4 . 5}$ & 0.5 & 0.33 & Bal. \\
Refined \& & $\mathbf{1 2}$ & 7.0 & 3.5 & 0.5 & 0.33 & Bal. \\
Modified & $\mathbf{6 . 0}$ & 3.5 & 0.5 & 0.33 & Bal. \\
\hline & $\mathbf{1 3}$ & $\mathbf{8 . 0}$ & 3.5 & 0.5 & 0.33 & Bal. \\
Grain & $\mathbf{1 4}$ & 7.0 & 3.5 & 0.5 & 0.33 & Bal. \\
Refined \& & $\mathbf{1 5}$ & 7.0 & $\mathbf{2 . 5}$ & 0.5 & 0.33 & Bal. \\
Modified & $\mathbf{1 6}$ & 7.0 & $\mathbf{4 . 5}$ & 0.5 & 0.33 & Bal. \\
& $\mathbf{1 7}$ & $\mathbf{6 . 0}$ & 3.5 & 0.5 & 0.33 & Bal. \\
\hline
\end{tabular}




\section{Section 3: Databases}

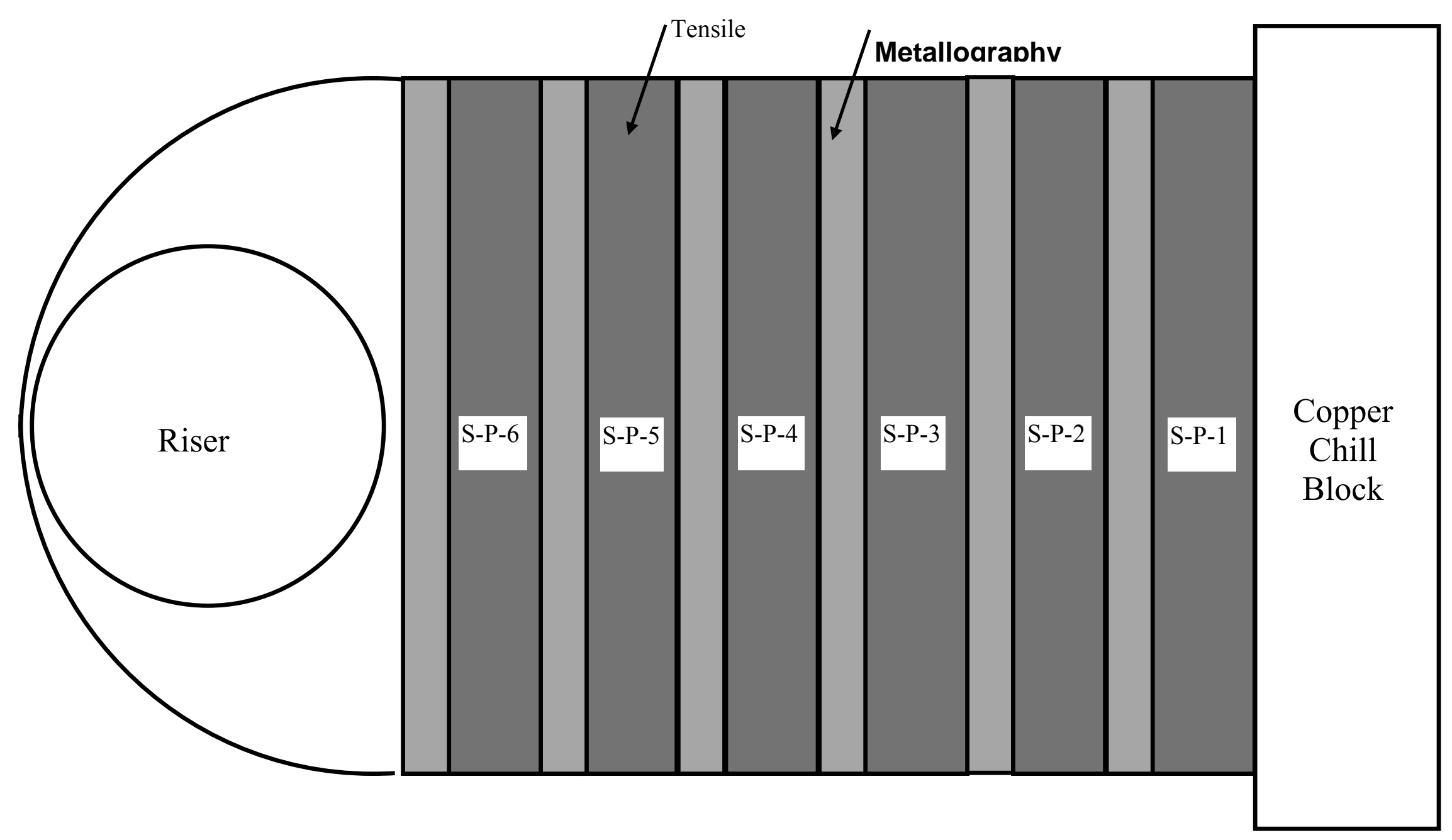

Figure 3.6-1. Schematic diagram of test plate casting showing placement of copper end chill and cylindrical riser. Coupons are cut for heat treatment, metallographic analysis, and mechanical testing, as shown. The last digit in the numbering scheme indicates the position of the coupon relative to the chill. S represents the alloy series and P represents the plate number. 


\section{Section 3: Databases}
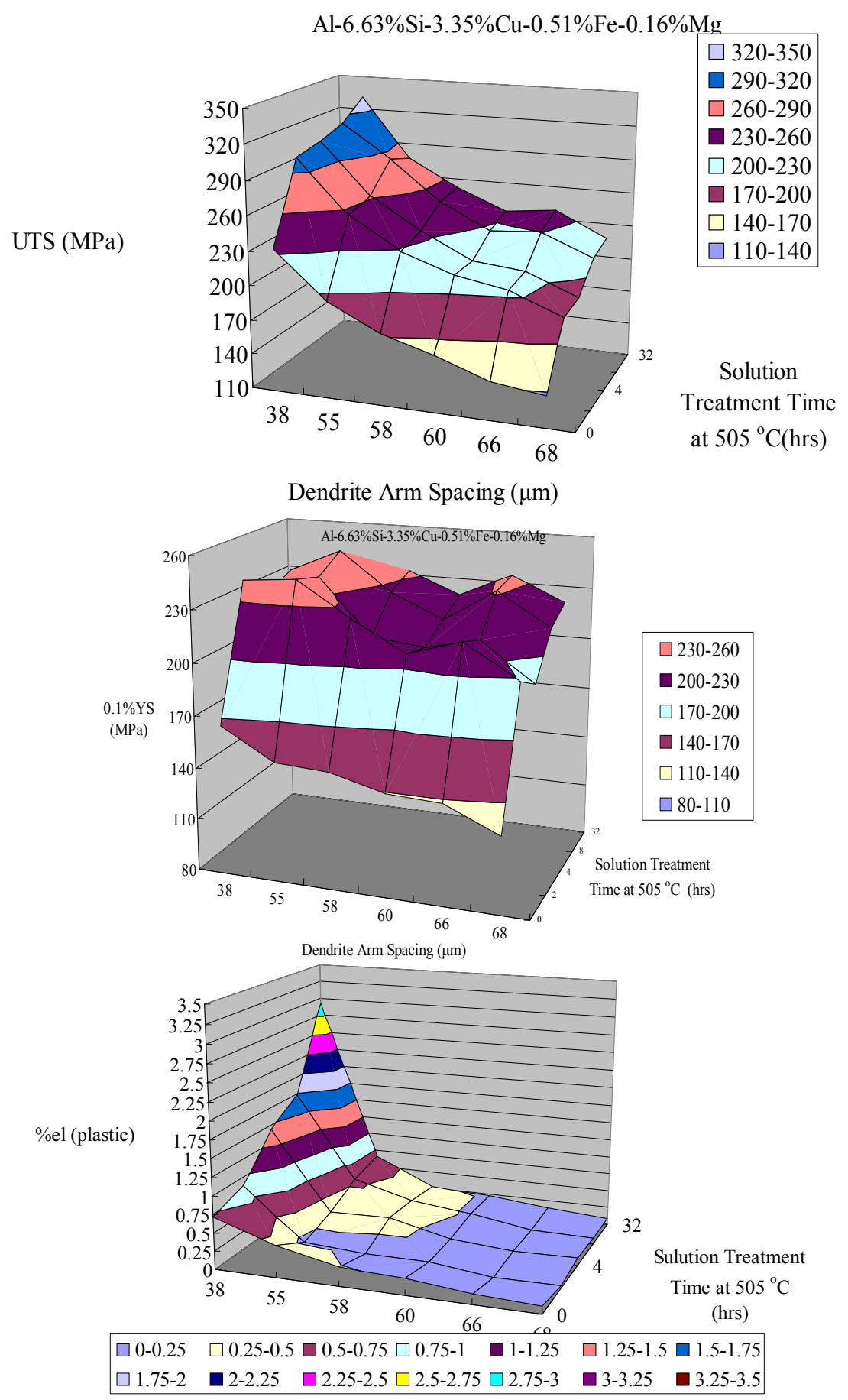

Figure 3.6-2. Tensile strength, yield strength $(0.1 \%$ offset $)$, \& plastic strain (\%) vs. solution treatment time \& dendrite arm spacing, nominal alloy 


\section{Section 3: Databases}

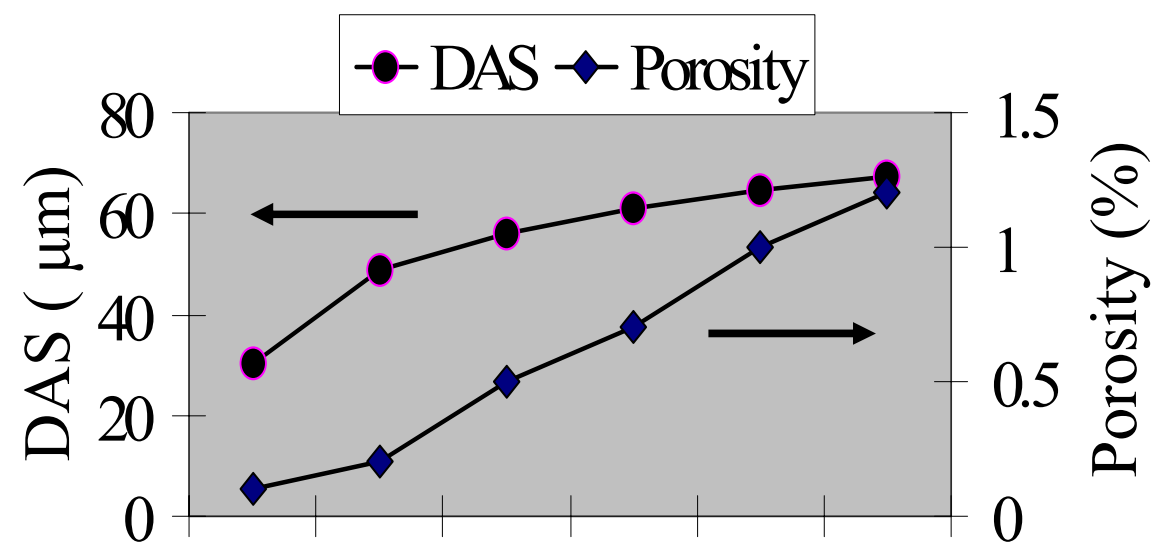

\section{$\begin{array}{llllll}13 & 51 & 89 & 127 & 165 & 203\end{array}$}

\section{Distance fromChill (mm)}

Figure 3.6-3. Variation in dendrite arm spacing and volume percent porosity with distance from the chill in end-chilled plate castings

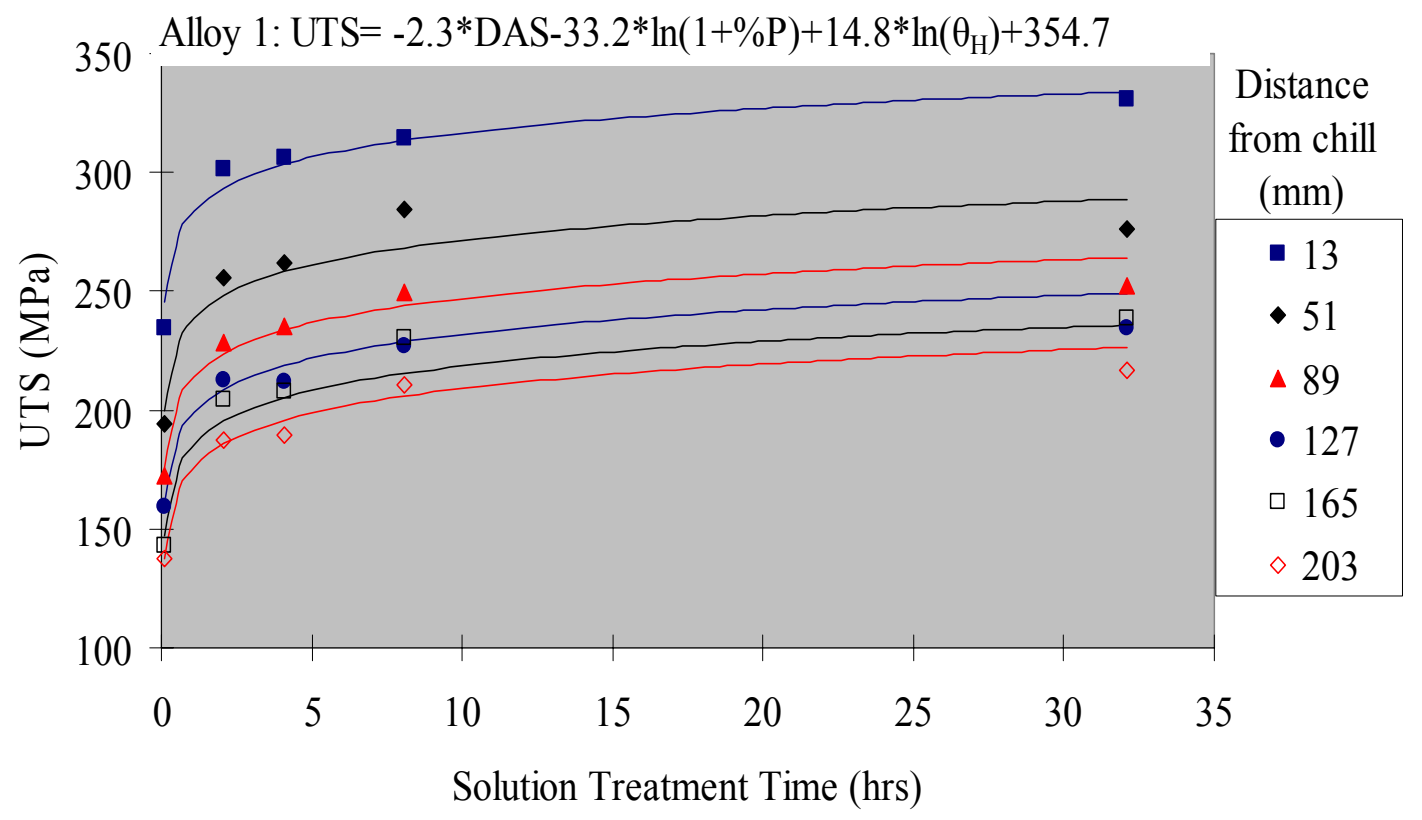

Figure 3.6-4. Comparison of measured tensile strength (points) and computed tensile strength (curve) vs. solution treatment time 


\section{Section 3: Databases}

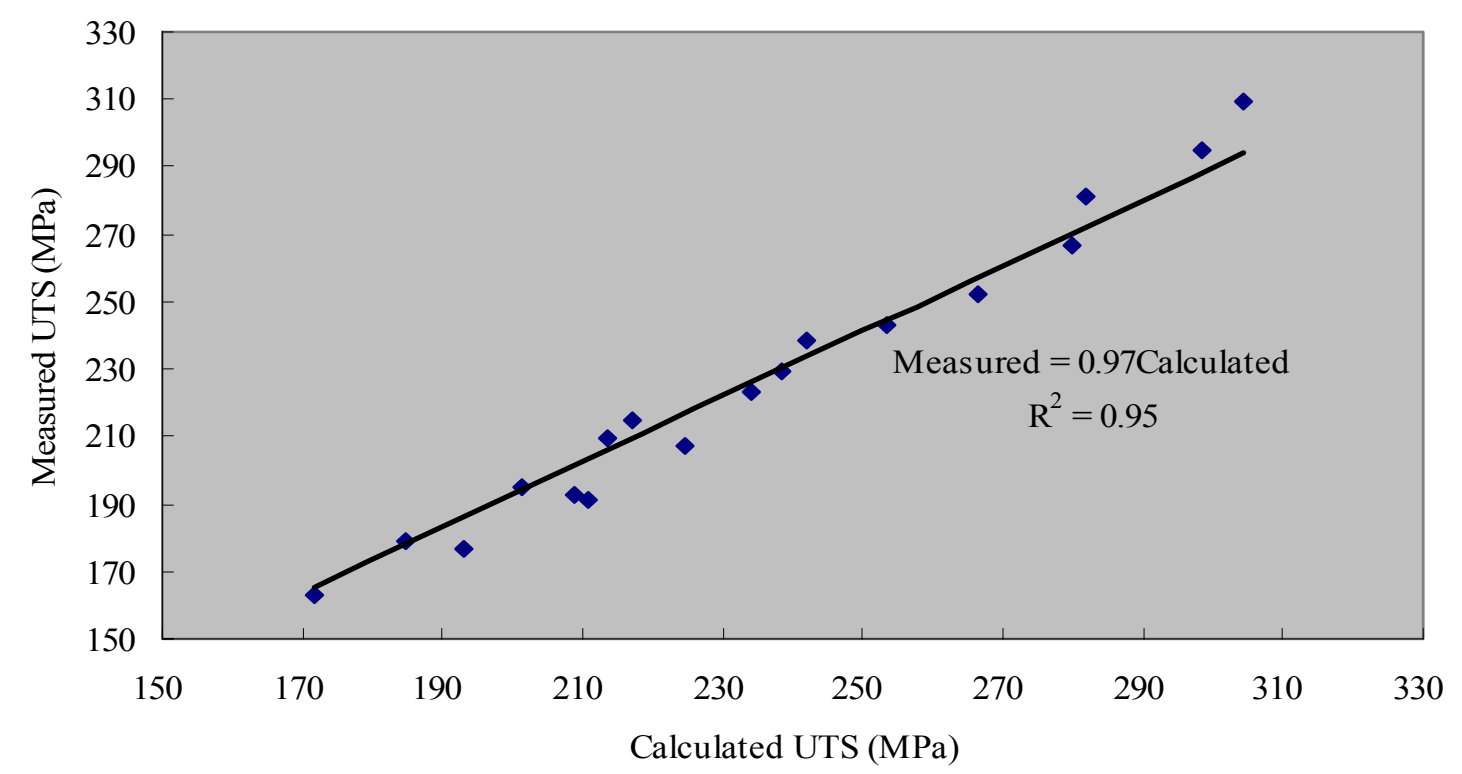

Figure 3.6-5. Comparison of measured tensile strength and computed tensile strength

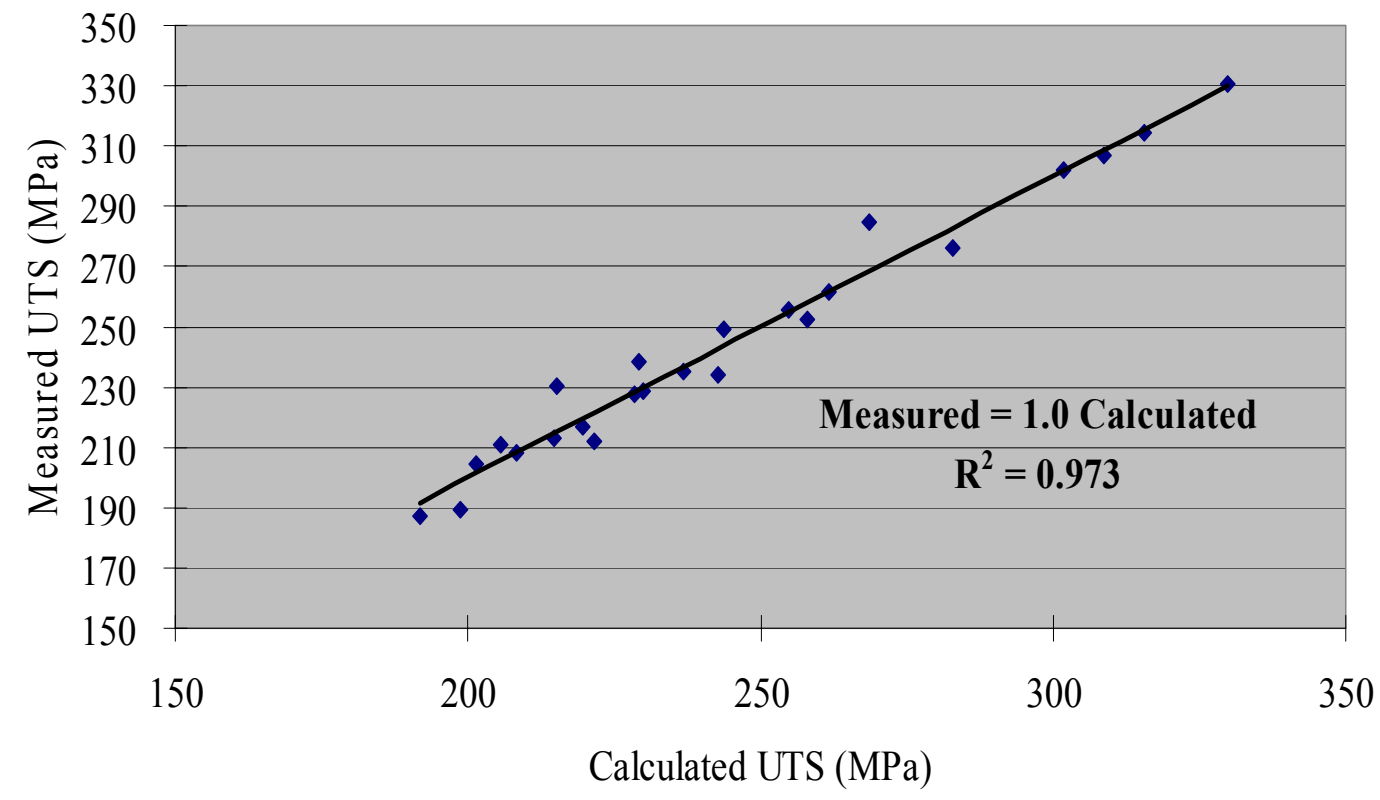

Figure 3.6-6. Validation: Comparison of measured tensile strength and predicted tensile strength for additional cast plates 


\section{Section 3: Databases}

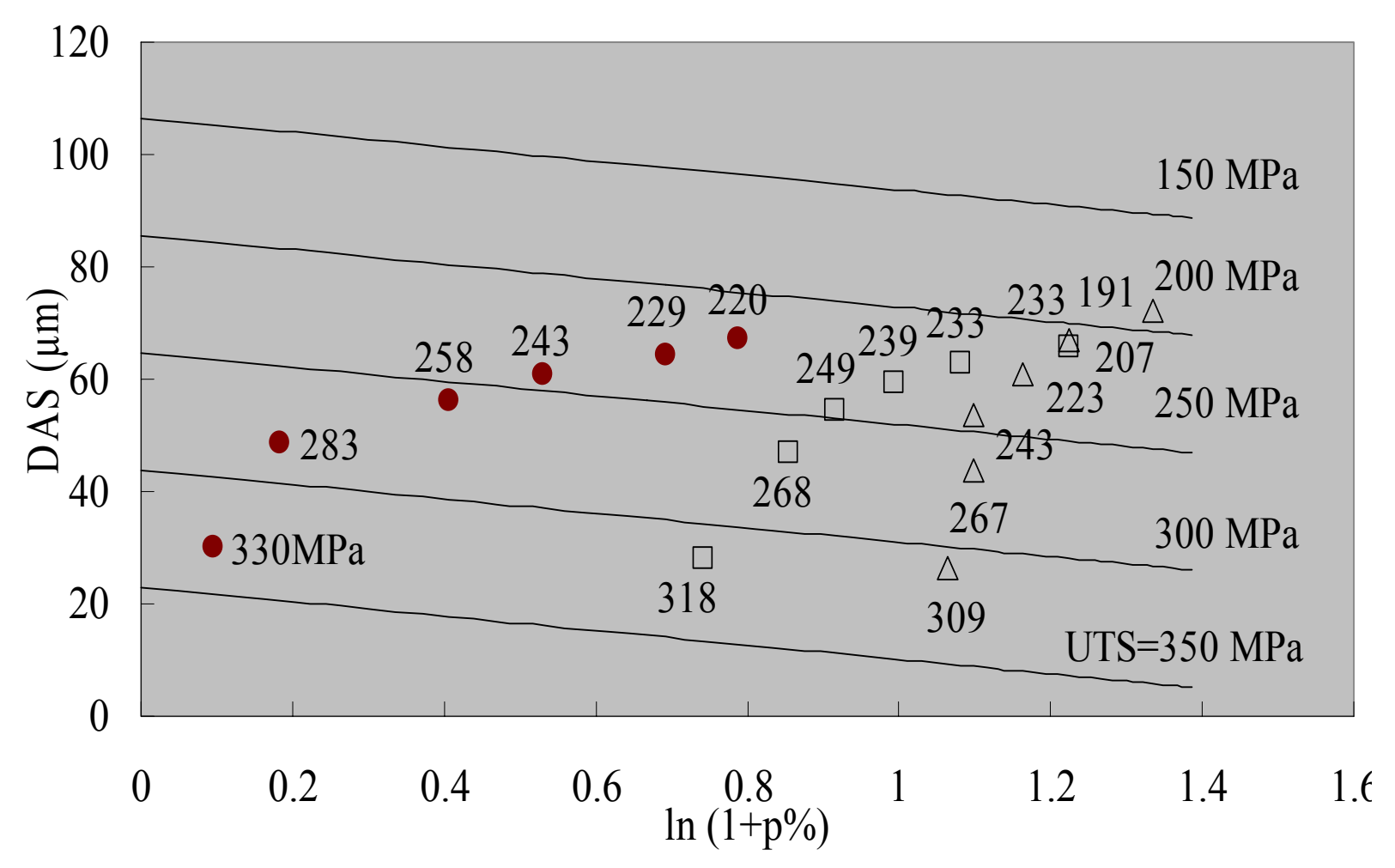

Figure 3.6-7. Comparison of measured tensile strength and isostrength lines computed with the empirical relation for as-cast dendrite arm spacing and volume percent porosity 


\section{Section 3: Databases}
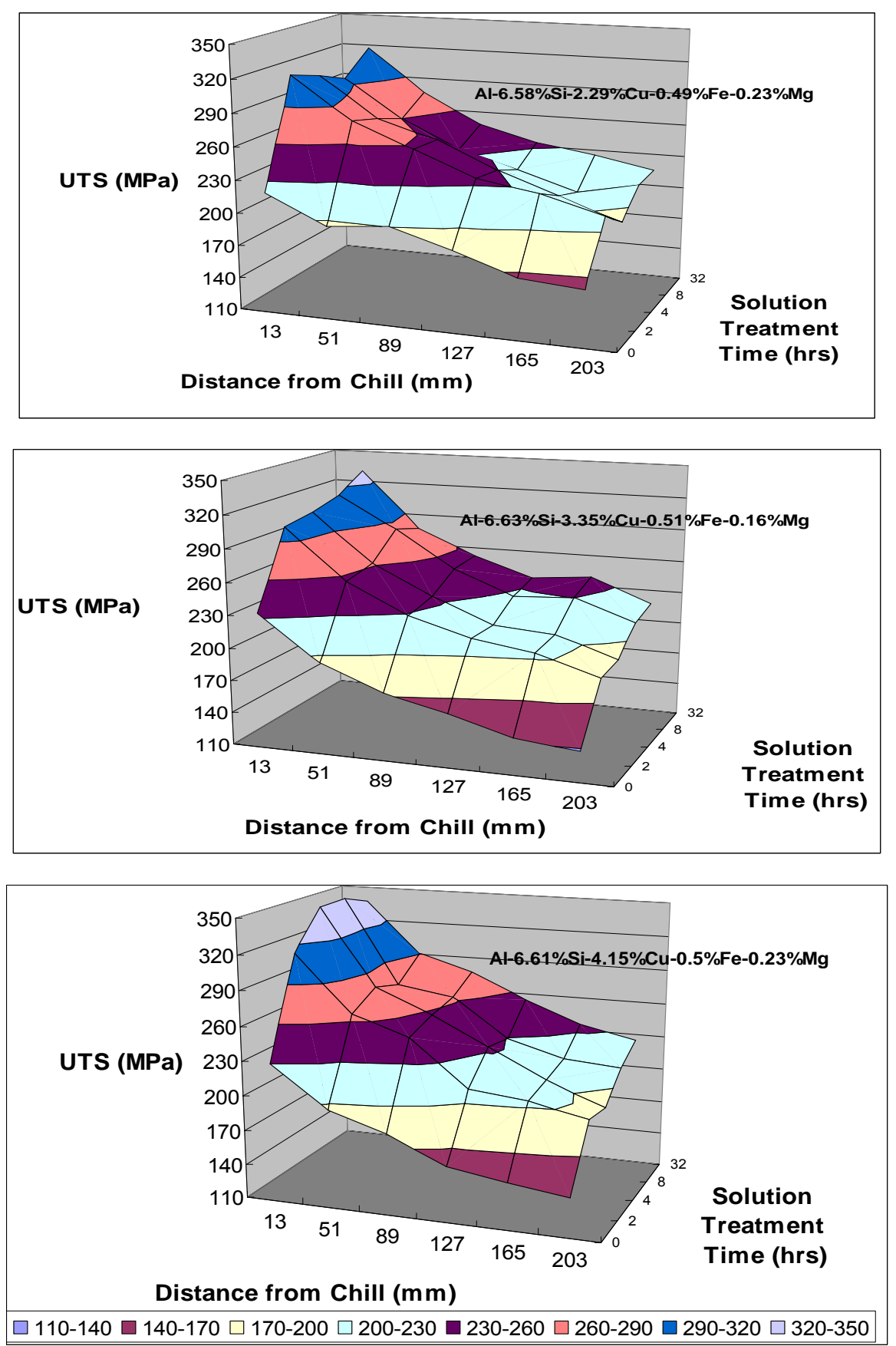

Figure 3.6-8. UTS vs distance from the chill and solution treatment time for alloys with low (top) and high (bottom) copper in comparison to the nominal composition (center) 


\section{Section 3: Databases}
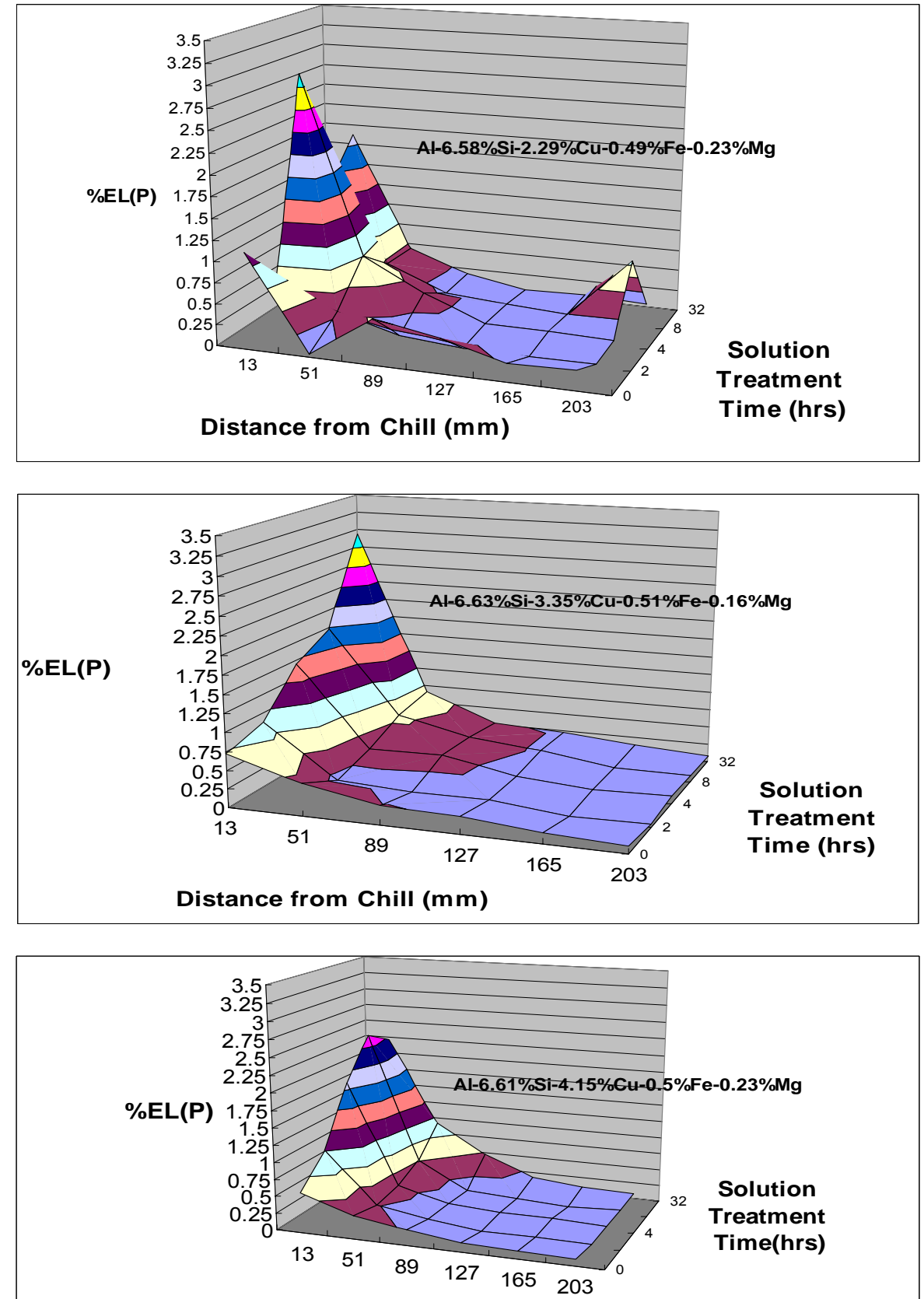

Distance from Chill $(\mathrm{mm})$

$\square$ 0-0.25 $\square$ 0.25-0.5 $\square$ 0.5-0.75 $\square$ 0.75-1 $\square$ 1-1.25 $\square$ 1.25-1.5 $\square$ 1.5-1.75

$\square$ 1.75-2 $\square 2-2.25 \quad \square 2.25-2.5 \square 2.5-2.75 \square 2.75-3 \quad \square 3-3.25 \quad \square 3.25-3.5$

Figure 3.6-9. \% elongation (plastic) vs distance from the chill and solution treatment time for alloys with low (top) and high (bottom) $\mathrm{Cu}$ in comparison to the nominal composition 


\section{Section 3: Databases}
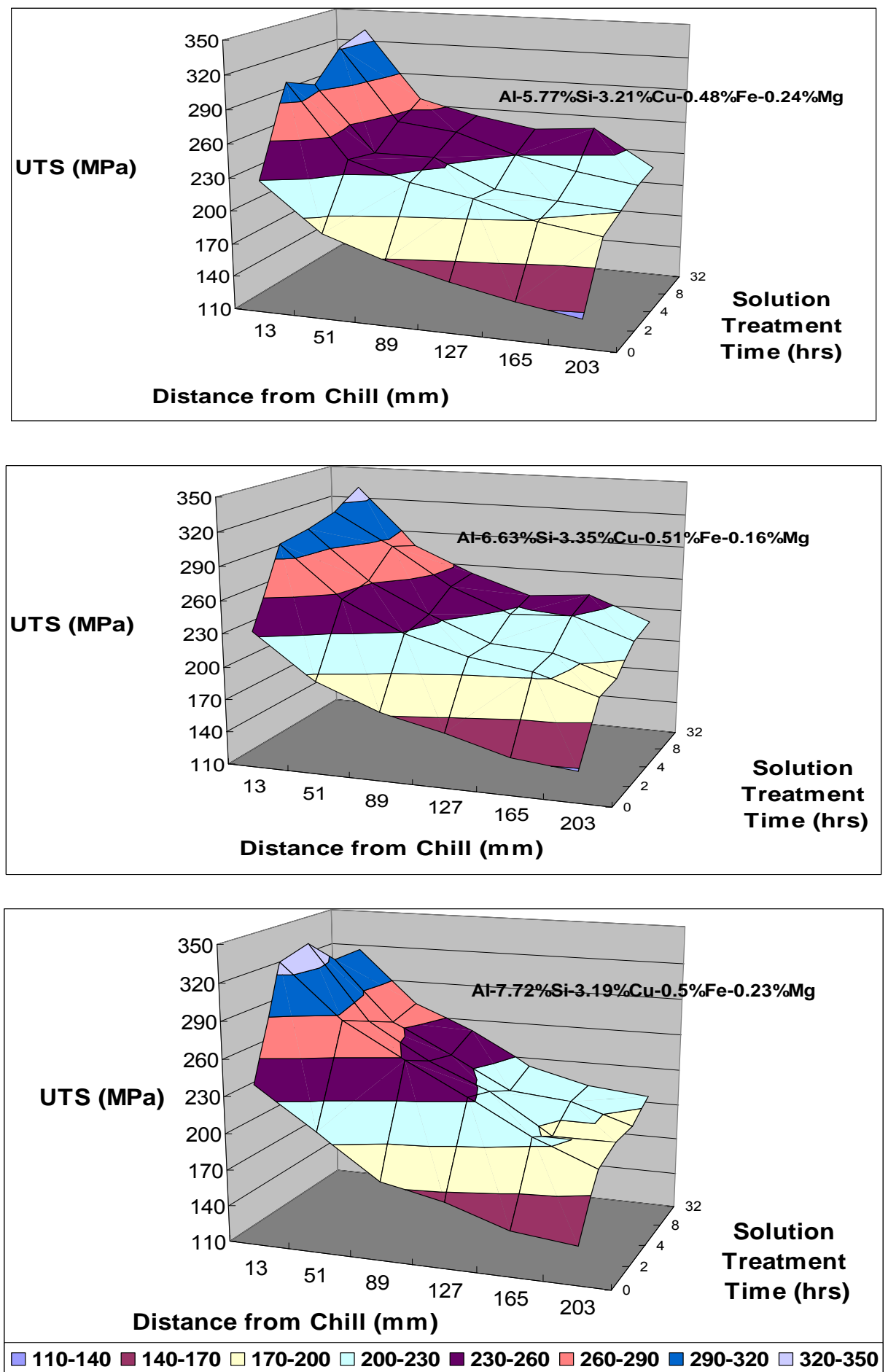

Figure 3.6-10. UTS vs distance from the chill and solution treatment time for alloys with low (top) and high (bottom) Si in comparison to the nominal composition (center) 


\section{Section 3: Databases}
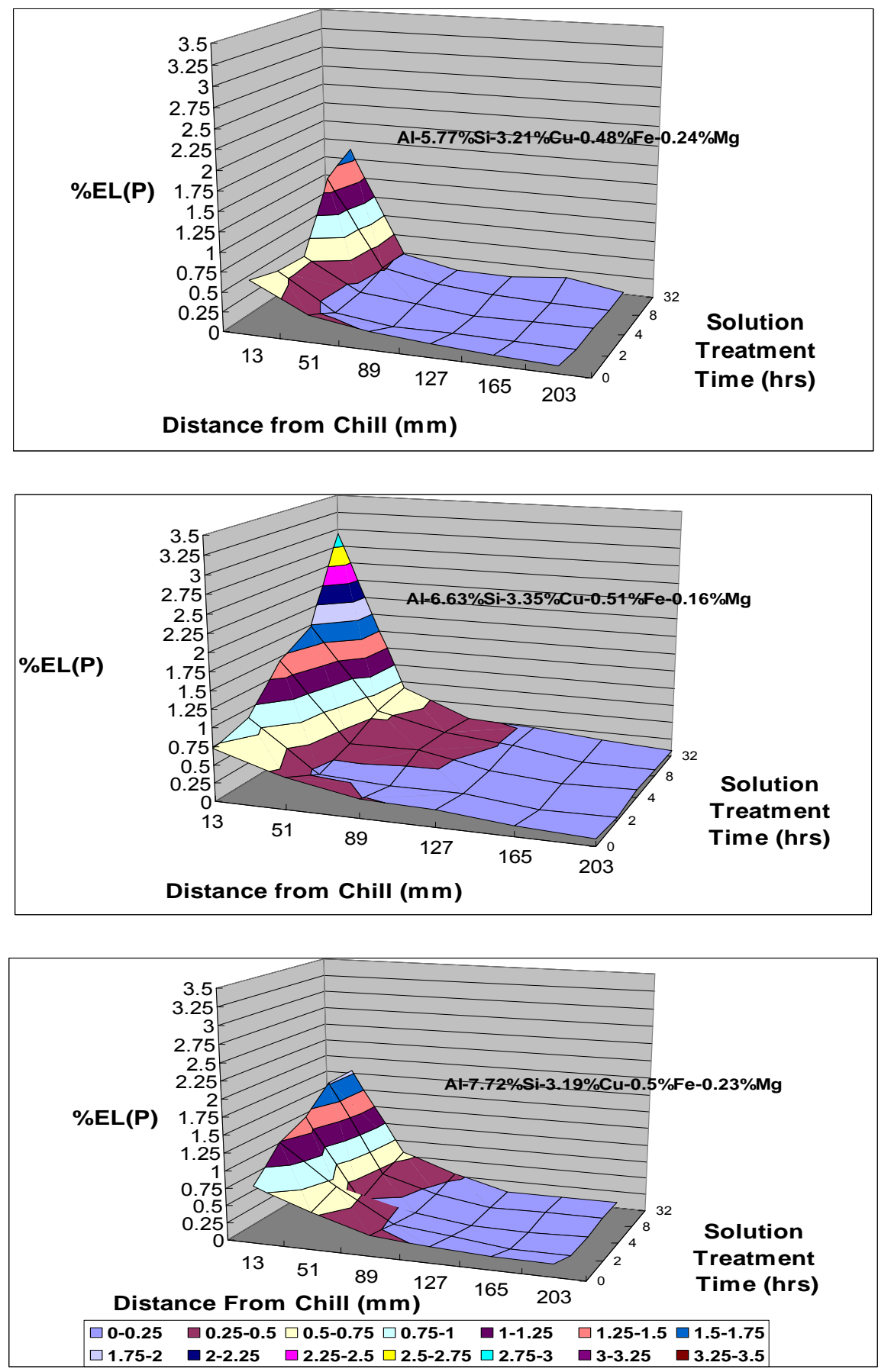

Figure 3.6-11. \% elongation (plastic) vs distance from the chill and solution treatment time for alloys with low (top) and high (bottom) Si in comparison to the nominal composition (center) 


\section{Section 3: Databases}
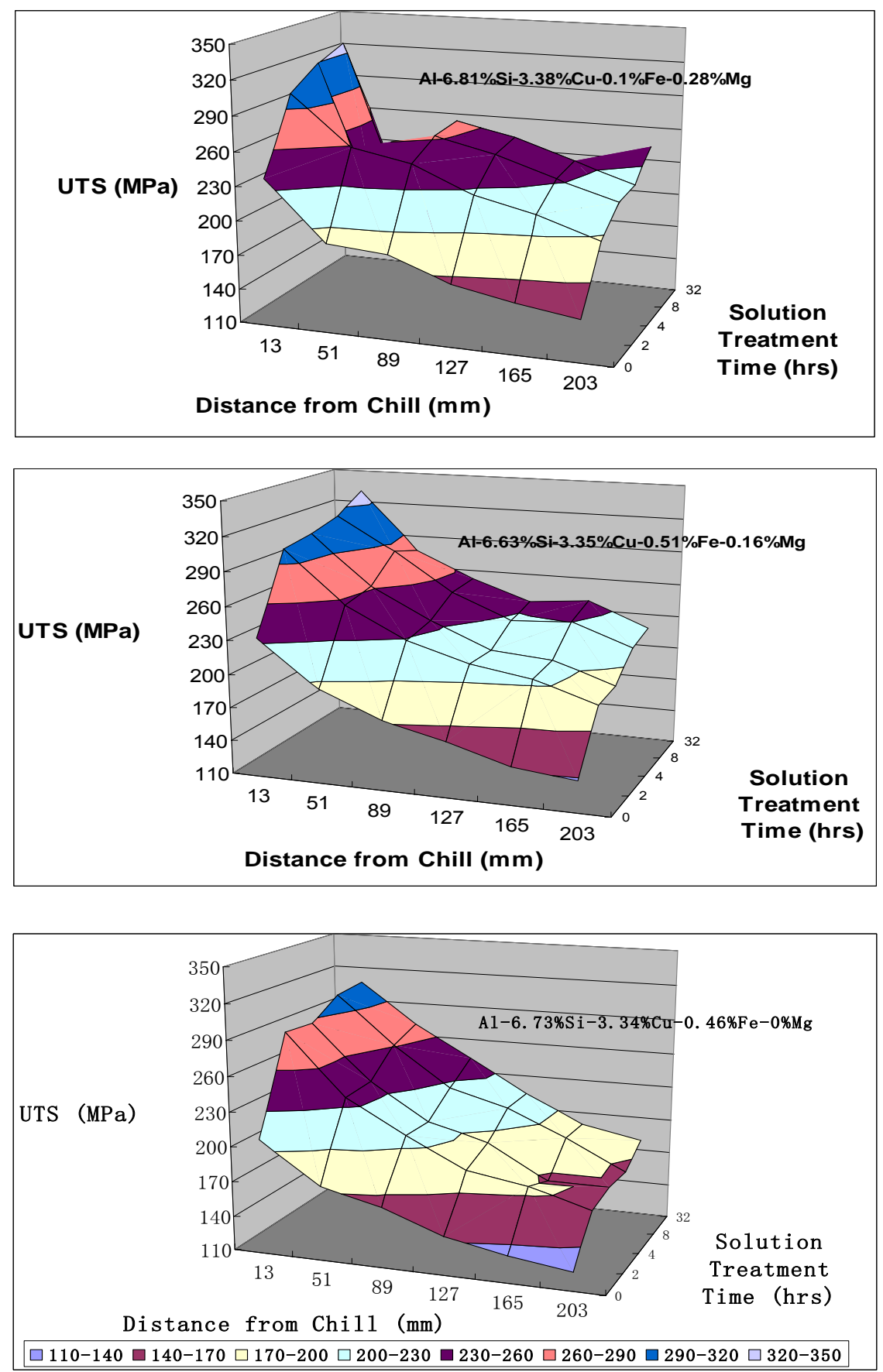

Figure 3.6-12. UTS vs distance from the chill and solution treatment time for alloys with low Fe (top) and low $\mathrm{Mg}$ (bottom) in comparison to the nominal composition (center) 


\section{Section 3: Databases}
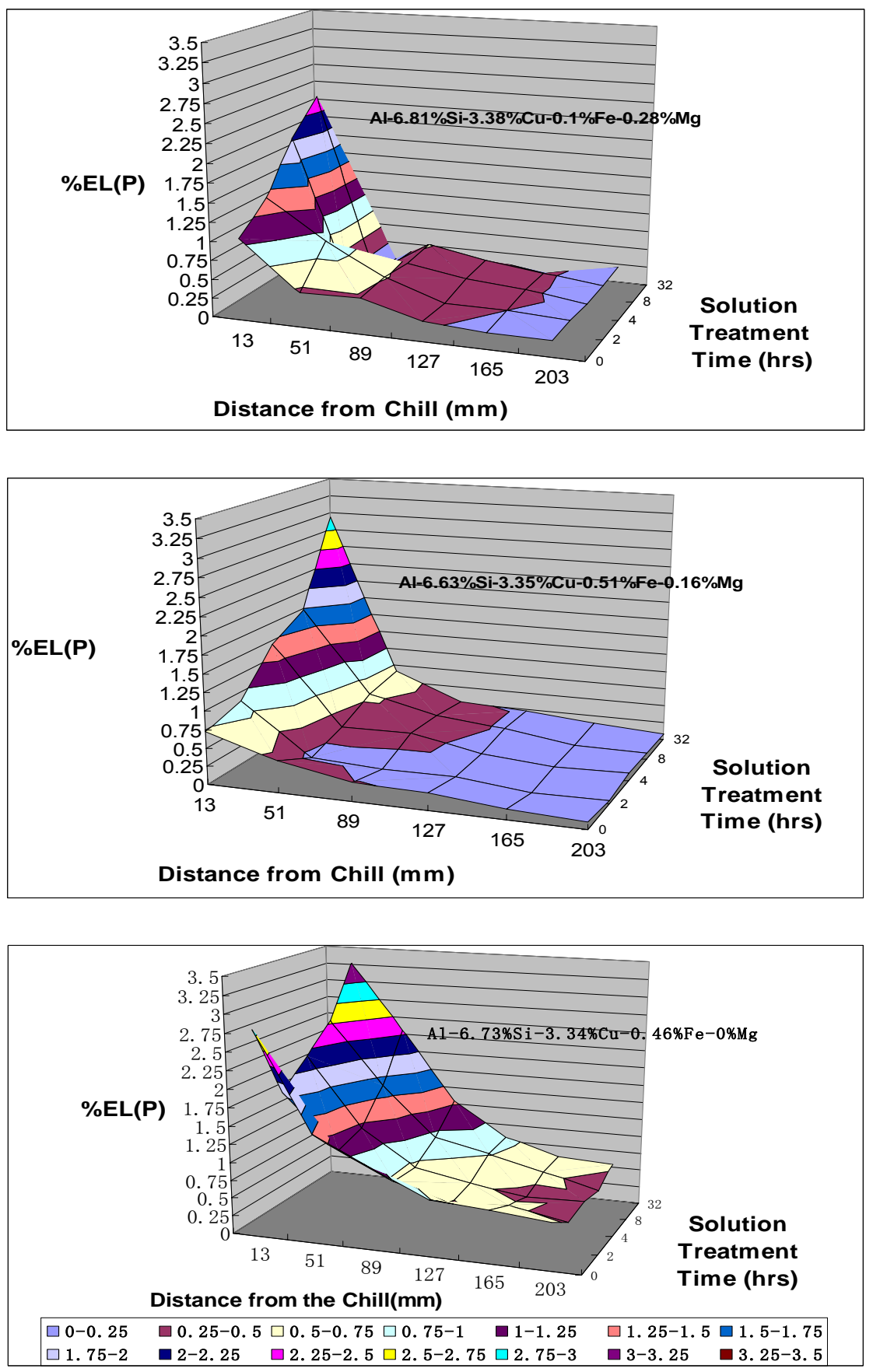

Figure 3.6-13. \%Elongation (plastic) vs distance from the chill and solution treatment time for alloys with low Fe (top) and low $\mathrm{Mg}$ (bottom) in comparison to the nominal composition (center) 


\section{Section 3: Databases}
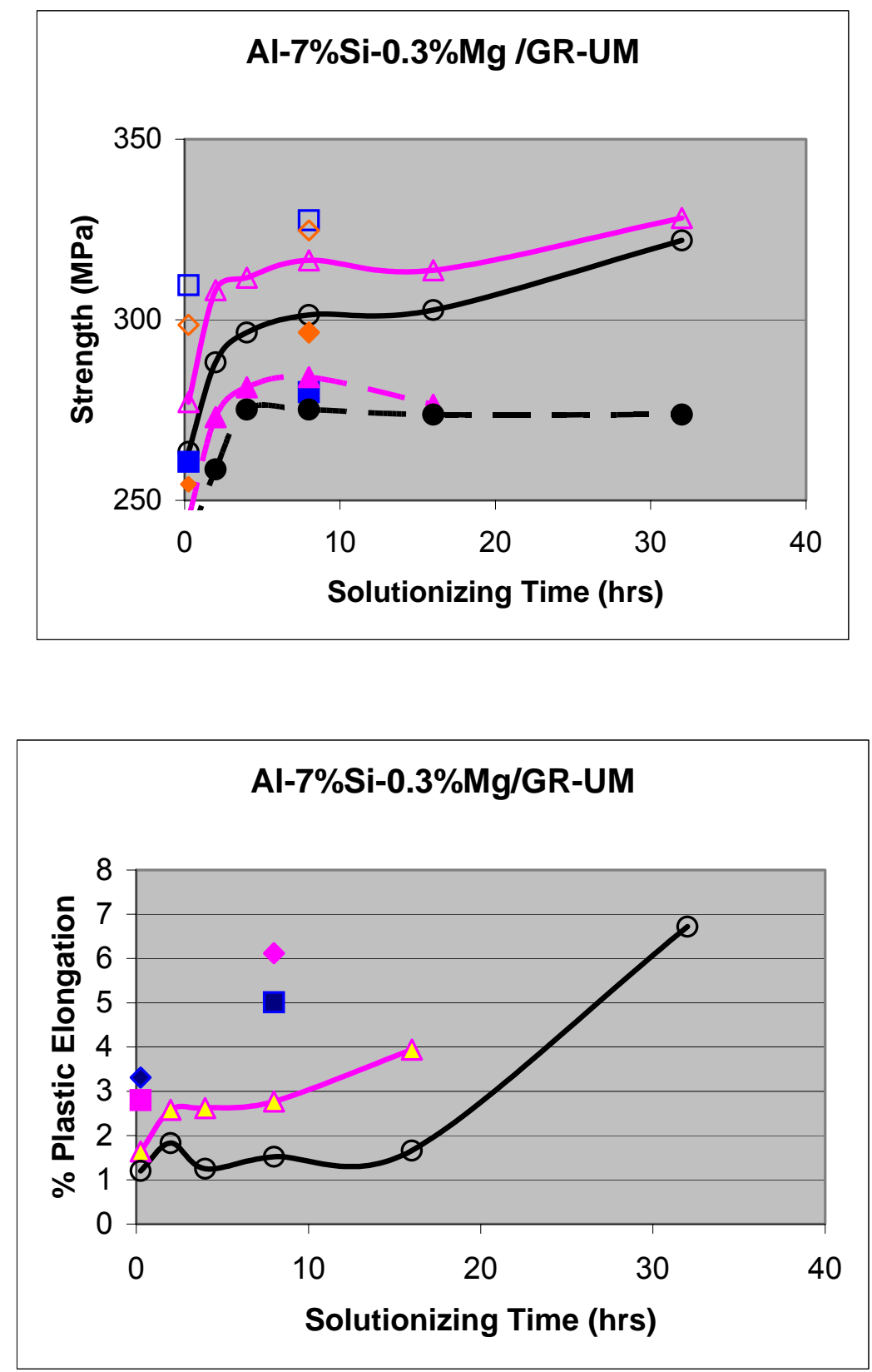

Figure 3.6-14. Tensile strength, yield strength (0.2\% offset-dashed line), and plastic strain (\%) vs. solution treatment time (hrs) for dendrite arm spacings: $17 \mu \mathrm{m}$ (squares), $35 \mu \mathrm{m}$ (diamonds), $44 \mu \mathrm{m}$ (triangles), $61 \mu \mathrm{m}$ (circles) 


\section{Section 3: Databases}

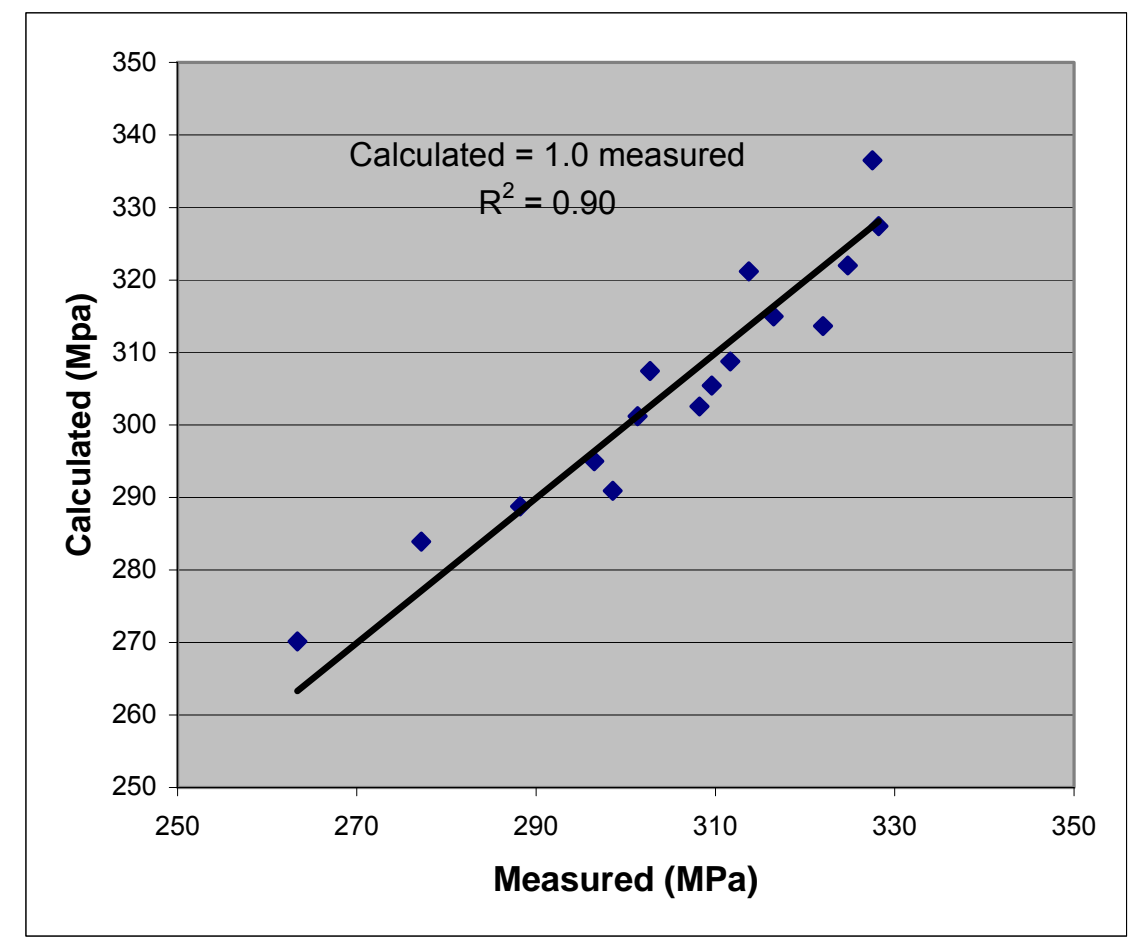

Figure 3.6-15. Comparison of computed and measured tensile strengths for nominal Al-Si-Mg alloy 


\section{Section 4: Integration of Thermal-Microstructure -Property Prediction}

The integrated model consists of three functionally independent while data-wise correlated modules: (a) thermal prediction; (b) alloy microstructure evolution prediction; and (c) mechanical property prediction. Thermal prediction is implemented first. Its simulation results are taken together with the alloy's as-cast conditions as input to the other two modules for predictions of microstructure and mechanical properties. In each of the three main modules, mathematical models and extended databases are developed. The general flowchart of the integrated software is shown in Figure 4-1. 


\section{Section 4: Integration of Thermal-Microstructure -Property Prediction}

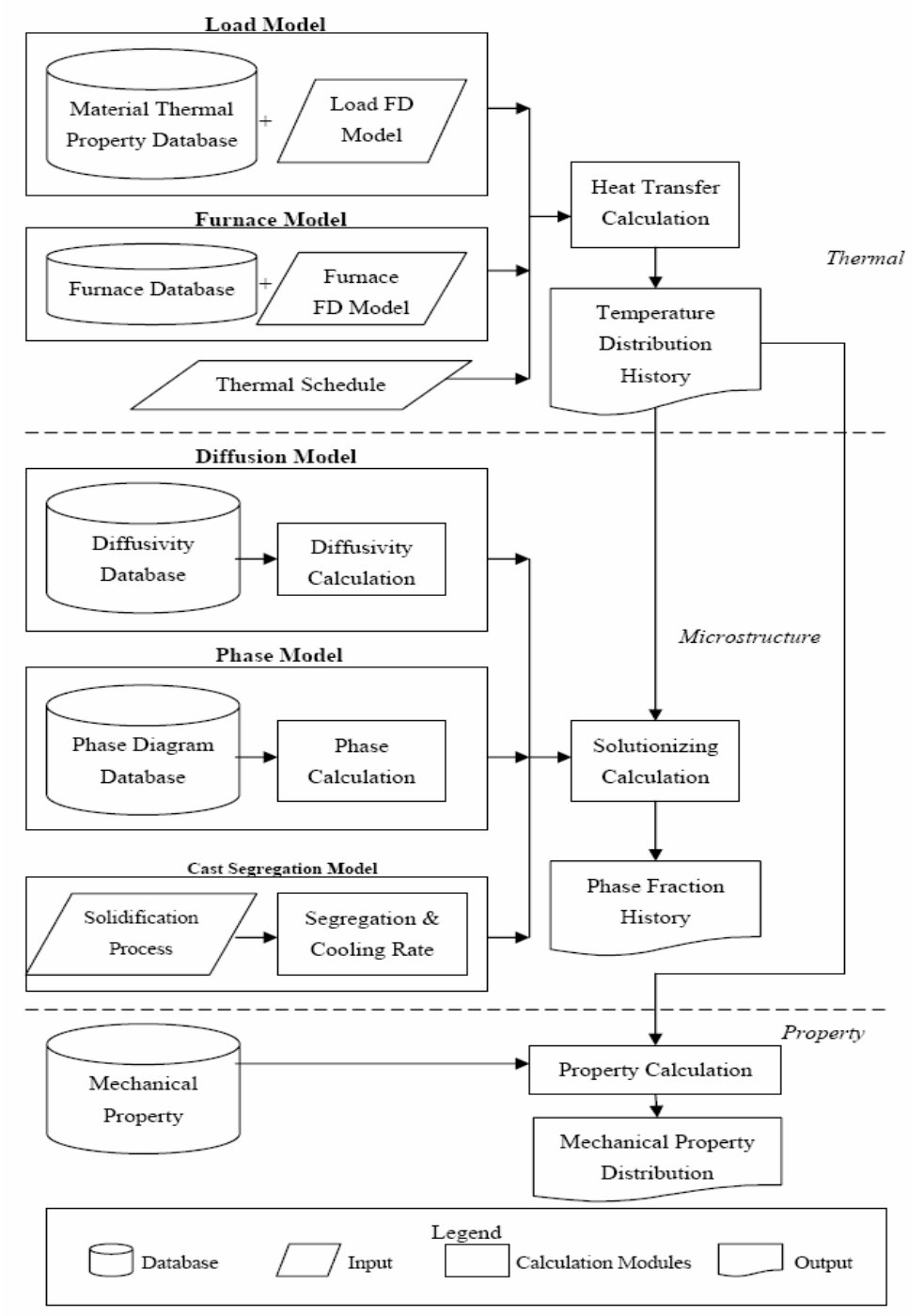

Figure 4-1. Flowchart of system overall structure

\subsection{Thermal prediction module}

The goal of the thermal prediction module is to predict the temperature $(\mathrm{T})$ profile of the entire furnace-load system in both time (t) and spatial domains, that is $\mathrm{T}=\mathrm{T}(\mathrm{t}, \mathrm{x}, \mathrm{y}, \mathrm{z})$. Load and furnace are modeled in a 3-D FD (Finite Difference) mesh file, so they can be of arbitrary shape. 


\section{Section 4: Integration of Thermal-Microstructure -Property Prediction}

Heat transfer is also calculated in 3-D using the FD method. Furnace data and workpiece material data are retrieved from relevant databases, and the furnace is assumed to be controlled using the PID control method.

The inputs to thermal prediction module include:

a. Furnace parameters and configurations, e.g. furnace power, heating type, geometry of the working space, geometries and materials for heaters, insulation walls, support, convection fan, etc. Such information can be input manually or imported directly from an updateable furnace database.

b. Thermal schedule. The expected furnace temperature cycle is to be specified.

c. The geometries and material specifications for parts, load pattern, and initial temperature. Materials' thermal properties are stored in an updateable materials database.

When the calculation is finished, the featured results are shown in diagrams, and comprehensive results are output to data files for later use by the microstructure and property prediction module. The output results of the thermal prediction module include: Temperature data of each part element at every time step, and temperature data of atmosphere and furnace elements at selected times.

\subsection{Alloy microstructure evolution prediction module}

The objectives of the numerical simulations are to predict the amount and distribution of phases, the solute distribution within the phases, and the average solute concentration across a characteristic dendrite arm during solidification, post-solidification cooling, and solution treatment in multi-phase, multi-component hypoeutectic alloy systems. The overall flow chart for the simulation routines is illustrated in Figure 4.2-1. The two major subroutines are:

a. CASTSEG: Simulates solidification and post-solidification cooling. 


\section{Section 4: Integration of Thermal-Microstructure -Property Prediction}

b. CASTSOLN: Simulates solution heat treatment.

The model chosen, equilibrium phase data, thermal process parameters, diffusion coefficients and microstructure parameters are included in the Preprocess module. Three models, 1-D plate, 1-D plate middle-growth (divorced eutectic) and 2-D plate, are available in the simulation. Once the program begins, it will ask the user to select the geometric model to be used in the simulation. After choosing a geometric model, simulation and process parameters have to be set. Users can do this job by themselves or take default values. Then corresponding CastSeg and CastSoln modules are executed.

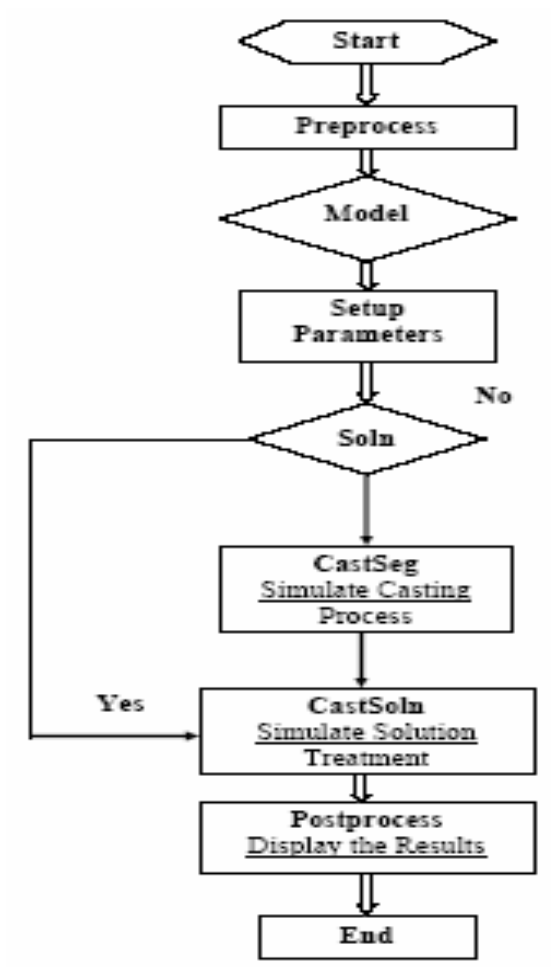

Figure 4.2-1. Flowchart of microstructure evolution simulation

Auxiliary programs, including the calculation of phase equilibrium, and solute diffusivity, facilitate the execution of the major subroutines. This is shown in Figure 4.2-2 . 


\section{Section 4: Integration of Thermal-Microstructure -Property Prediction}

1. PhaseCalc computes solid phase compositions at interfaces;

2. ThermalCycle provides the temperature variation versus temperature;

3. DiffCalc computes the diffusion of solute in the different phases;

4. SolnCalc provides the solution treatment temperature and time.

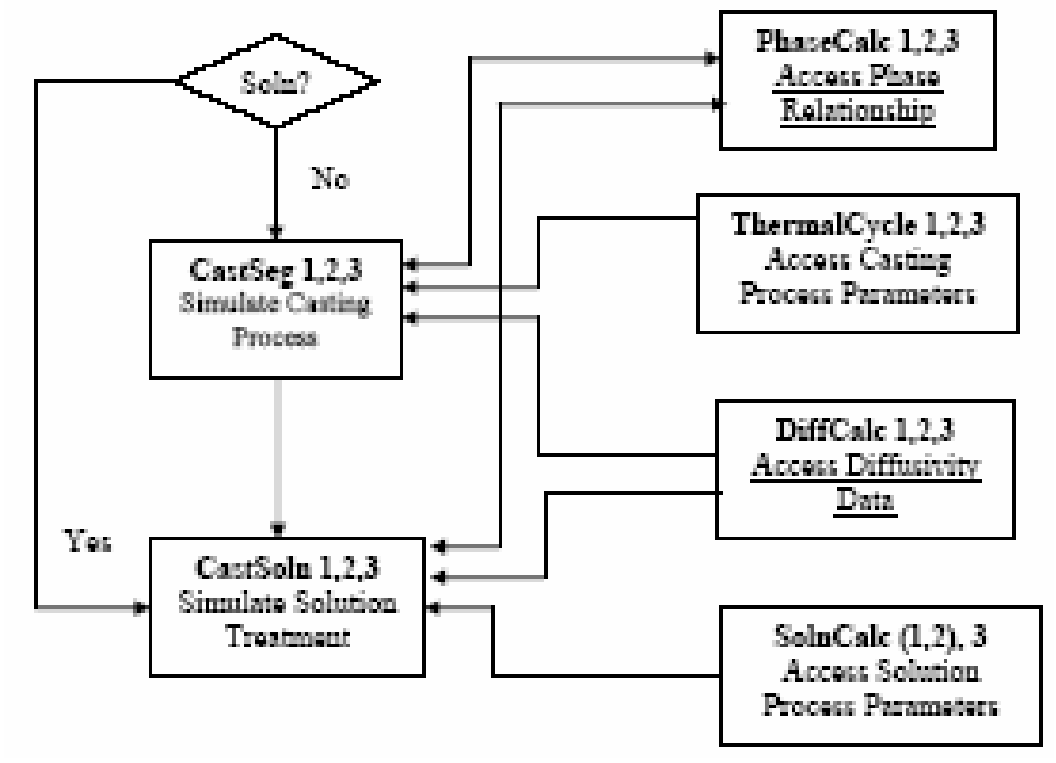

Figure 4.2-2. Relationship between main module and sub-module

Because the simulation covers microstructure evolution and diffusion in the solid, four kinds of data are input to the program, as indicated in Figure 4.2-3:

a. Equilibrium phase data.

b. Microstructure parameters- in particular, the secondary dendrite arm spacing.

c. Casting process thermal cycle parameters.

d. Solution treatment thermal cycle data. 


\section{Section 4: Integration of Thermal-Microstructure -Property Prediction}

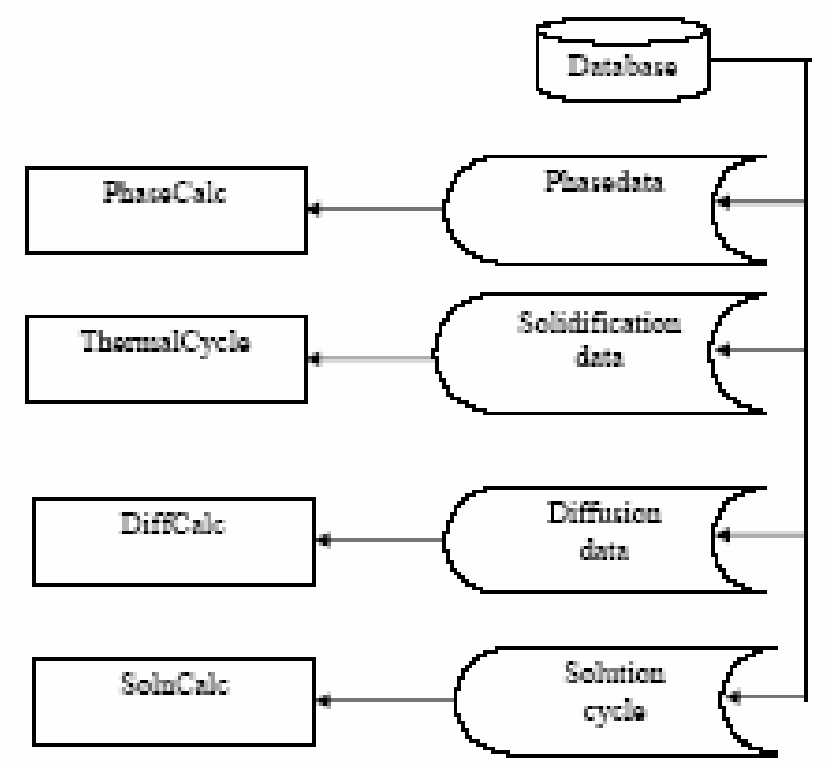

Figure 4.2-3. Relationship between sub-module and database

At the beginning of executing the simulation, users make their model options. After decisions are made, the physical parameters are initialized for the program. This is included in Figure 4.2-4 .

a. Setup DAS data: (a.) The program will ask the dendrite arm spacing on the screen so that users can select the default value or input their values. (b.) Set up the number of slices (resolution) in the volume element.

b. Setup diffusivity data. Pre-coefficient and activation energy in the diffusion coefficient equation for each element are input from stored data or input by the user. If off-diagonal terms are available and significant, they can be included in the preprocess;

c. Set criterion for Fourier number for diffusion using FDM (finite difference method).

d. Setup casting data. Users can initialize solidification time and cooling time according to their experimental results or from the results of casting simulation software. 


\section{Section 4: Integration of Thermal-Microstructure -Property Prediction}

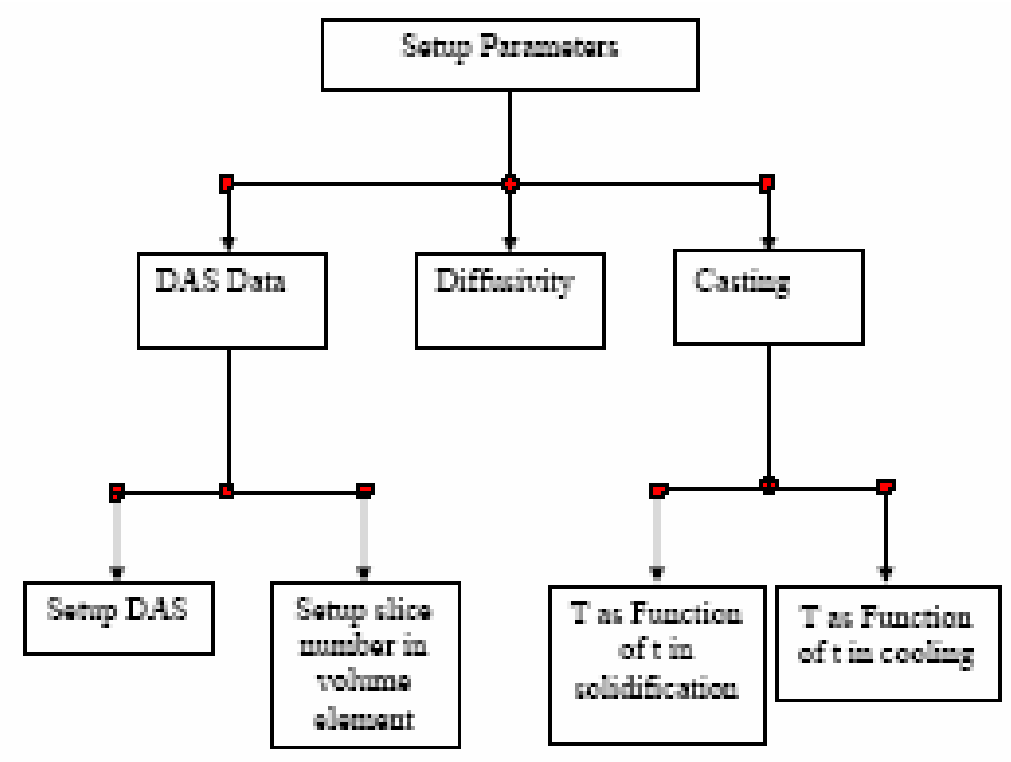

Figure 4.2-4. Flowchart for setup parameters

\subsection{Mechanical property prediction module}

Design rules relating mechanical properties of cast $\mathrm{Al}-\mathrm{Si}-\mathrm{Cu}-\mathrm{Mg}$ and $\mathrm{Al}-\mathrm{Si}-\mathrm{Mg}$ casting alloys to a minimum set of process and microstructure parameters have been presented in section 3.6. Tensile strength at a specified location can be predicted from a validated equation, specific to the alloy composition, aging practice, and to the foundry's standard practice. Input to the equation are (1.) dendrite arm spacing (das) or local solidification time $\left(\theta_{\mathrm{f}}\right)$; (2.) volume percent of porosity $(\% \mathrm{P})$ or dissolved hydrogen in the melt $\left(\mathrm{H}_{2}\right)$ at entry to gating system; and (3.) solution treatment time $\left(\theta_{\mathrm{S}}\right)$. The dendrite arm spacing (das) is proportional to the scale of the as cast microstructure and is determined by the local cooling rate of the casting through the solidification range. The volume percent porosity $(\% \mathrm{P})$ depends on amount of hydrogen dissolved in the molten alloy at the time of casting and on the local cooling rate and thermal gradient. The solution treatment time $\left(\theta_{\mathrm{S}}\right)$ is a control parameter for the heat treatment thermal cycle. The data to the design rule can be input from casting heat transfer (and fluid flow) simulation software or by the user from measurement and/or specified values. Development of 


\section{Section 4: Integration of Thermal-Microstructure -Property Prediction}

a validated design rule to fit a specific foundry's standard practice can follow the approach described in section 3.6.1.

The correlation of strength properties to this set of three process and microstructure parameters is surprisingly good. As an example, the equation used for solution treatment of $\mathrm{Al}-\mathrm{Si}-\mathrm{Cu}-\mathrm{Mg}$ alloys at $505^{\circ} \mathrm{C}$ is shown in the box in Figure 4.3-1. The slope of one on the graph demonstrates the agreement between predictions of the model and measured strength values. The empirical correlation is used as a design rule to predict mechanical properties.

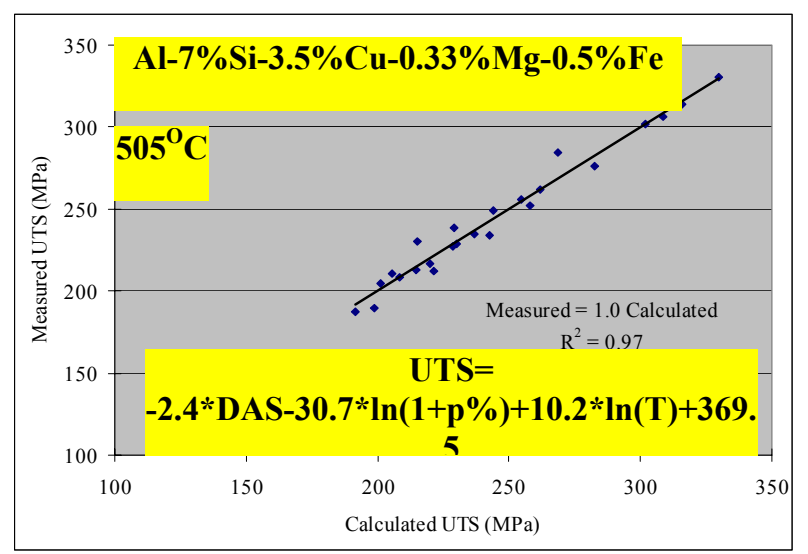

Figure 4.3-1. Correlation of measured tensile strength and the calculated one using given equation

\subsection{Case Study}

A case study, including experiment and its simulation, was done to validate the system. The workpiece being heated was as-cast Al-alloy 319 (Al-7Si-3.5Cu-0.5Mg) tensile bar, with the geometry and dimensions shown in Figure 4.4-1. The bars were loaded crisscross in 7 layers with 4 bars per layer, as shown in Figure 4.4-2, in a Lindberg Temperite electric furnace, model 11-MT-121812-14, rated at $4.5 \mathrm{~kW}$. The load was located at the bottom center of the furnace working space, as in Figure 4.4-3. Thermocouples (TC) were located as follows: one TC on top corner of the load representing the fast heated point (Note circle in Figure 4.4-4a); one at bottom center of the load representing the slowly heated point (Note circle in Figure 4.4-4b). Original 


\section{Section 4: Integration of Thermal-Microstructure -Property Prediction}

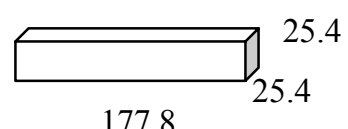

Figure 4.4-1. Workpiece shape and dimension (Unit: mm)

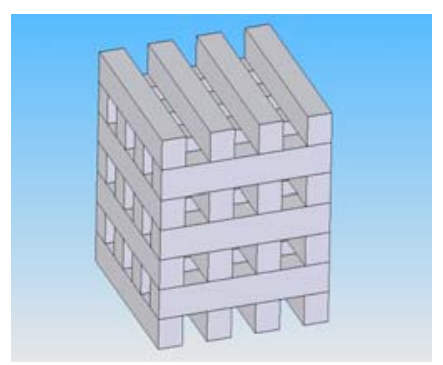

Figure 4.4-2. Load setup

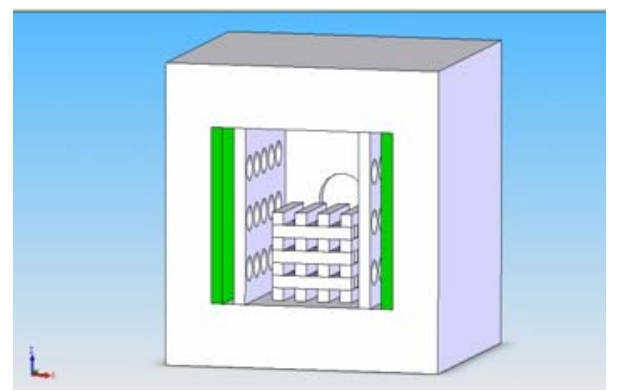

Figure 4.4-3. Relative position of furnace and load

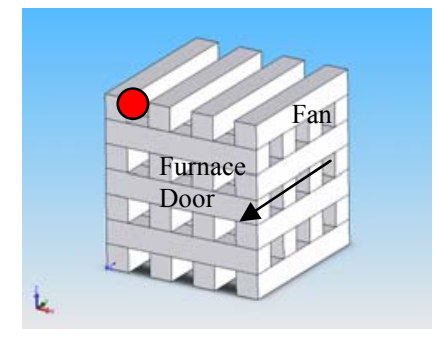

a: top thermal couples

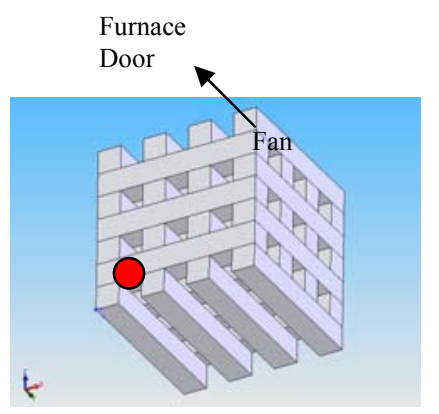

$b$ : bottom thermal couples

Figure 4.4-4. Positions of thermal couples 


\section{Section 4: Integration of Thermal-Microstructure -Property Prediction}

thermal schedule was set as in Table 4.4-1, where soaking time is 8 hours. The meshing step used for FD analysis in this case was $25.4 \mathrm{~mm} * 25.4 \mathrm{~mm} * 25.4 \mathrm{~mm}$.

Table 4.4-1. Thermal schedule

\begin{tabular}{|c|c|}
\hline Time $(\min )$ & $\begin{array}{c}\text { Temperature } \\
\left({ }^{\circ} \mathrm{C}\right)\end{array}$ \\
\hline 0 & 21 \\
\hline 40 & 505 \\
\hline 520 & 505 \\
\hline
\end{tabular}

The temperature distribution of the load at $54 \mathrm{~min}$ is presented in Figure 4.4-5. Since the temperature history computed for every element in the whole furnace-load system was recorded, temperature distribution of either the load, or furnace wall, or heating element, or support can be chosen for display.

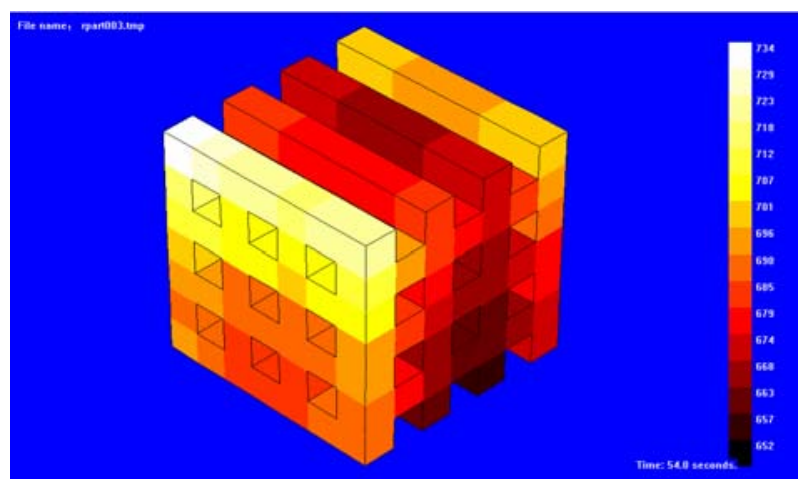

Figure 4.4-5. Temperature field at $54 \mathrm{~min}$

In Figure 4.4-6, the difference in temperature histories of fast and slowly heated points are shown, and the simulation temperatures are compared with the experimental thermal cycles. For both fast and slowly heated elements, the two sets of results match well except for the beginning. Initially, the calculated temperature rises more slowly than the experimental one. The primary cause of this deviation is the limit of the meshing step. With the presence of this limit, thin-wall 


\section{Section 4: Integration of Thermal-Microstructure -Property Prediction}

components (air duct shell in this case study) will be thicker in the model than actual. In this case, during the beginning period of heating, the heat input from the heating element will be largely absorbed by the air duct shell rather than the workpiece, which in turn delays the temperature rise of the workpiece. However, it has little effect on the calculation of total heating time.

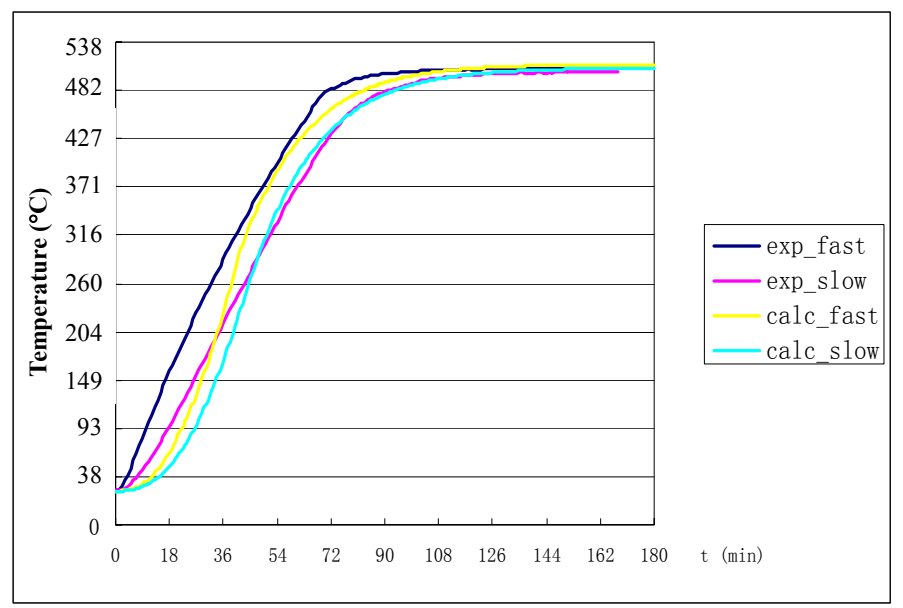

Figure 4.4-6. Comparison between experimental and calculated temperature profiles

\section{Microstructure}

The temperature history data of the points in the workpiece with the fastest and slowest heating rate were selected as the input to the alloy microstructure evolution prediction module (CastSoln). The as-cast dendrite arm spacing (DAS) of the Al-Si-Mg-Cu alloy in the heat treated coupons is $38 \mu \mathrm{m}$. Strengthening developed by the solutionizing, quench, and aging heat treatment depends on precipitation of intermetallic phases with $\mathrm{Cu}, \mathrm{Si}$, and $\mathrm{Mg}$. In the as-cast microstructure the $\mathrm{Cu}$ and $\mathrm{Mg}$ are tied up in coarse intermetallic particles of $\theta$-phase and Q-phase. All of the $\theta$-phase and most of the Q-phase will dissolve with a sufficiently long solution treatment. Figure 4.4-7 and 4.4-8 show the progress of dissolution of $\theta$-phase and Q-phase as predicted by the CastSoln module. The difference in time between the two points is negligible to achieve complete dissolution of $\theta$-phase and dissolution of the noneqilibrium fraction of Q-phase. 


\section{Section 4: Integration of Thermal-Microstructure -Property Prediction}

Importantly, though, effective dissolution of coarse solidification phases can be achieved within 2 hours of solutionizing. (Figure 4.4-7 and 4.4-8)

Weight Percent of 6 durizg Rehent aud Solution Treat Proces

(Al-7\%Si-35\%Cu-0.5\%Mg DAS=38 Microus)

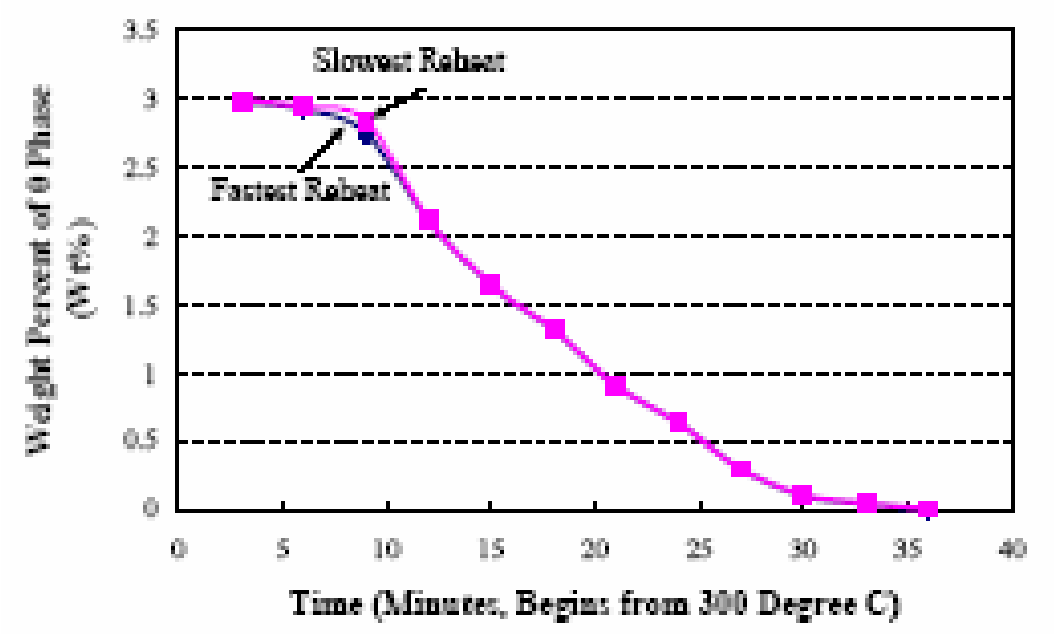

Figure 4.4-7. Variation of weight fraction of $\theta$ phase during solution treatment

Weight Percest of Q Phase during Reheat and Solucion

Treacuest Process

(Al-7\%Si-35\%Cu-0.5\%Mg DAS=38 Microms)

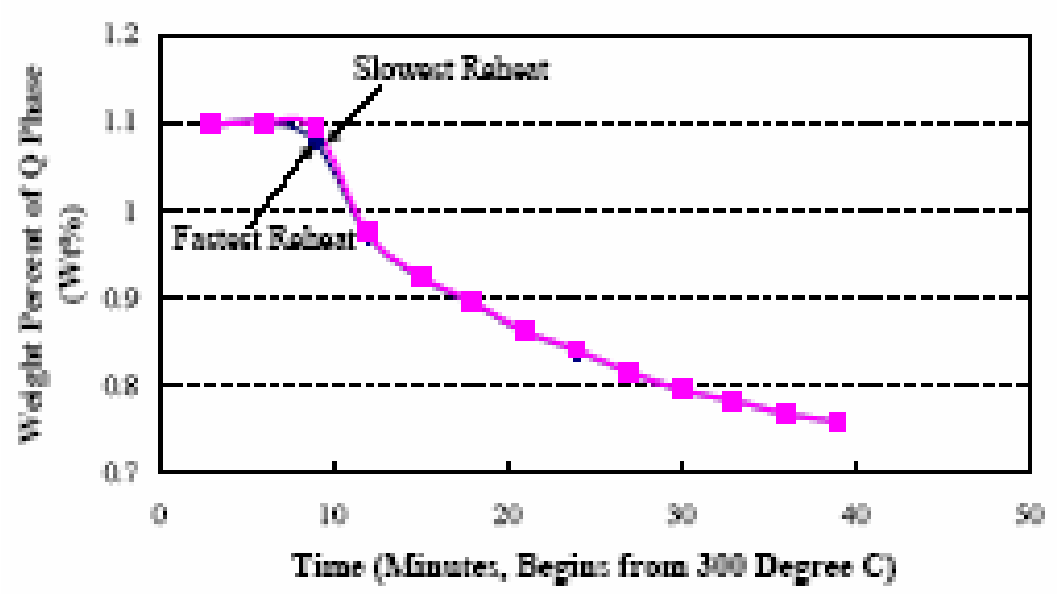

Figure 4.4-8. Variation of weight fraction of Q phase during solution treatment 


\section{Section 4: Integration of Thermal-Microstructure -Property Prediction}

\section{Property}

Based on the casting conditions and thermal history, mechanical properties, specifically ultimate tensile strength (UTS), yield strength (YS) and plastic elongation (\%el) can be predicted. (Figures 4.4-9-4.4-11) The properties predicted for the location with the highest heating rate do not differ significantly from the properties at the location with the lowest heating rate. The user can decide which combination of properties best suits the application and specify the heat treatment to reliably achieve those properties. Two hours of solution heat treatment would be sufficient to achieve the combination of mechanical properties commonly specified for this alloy.

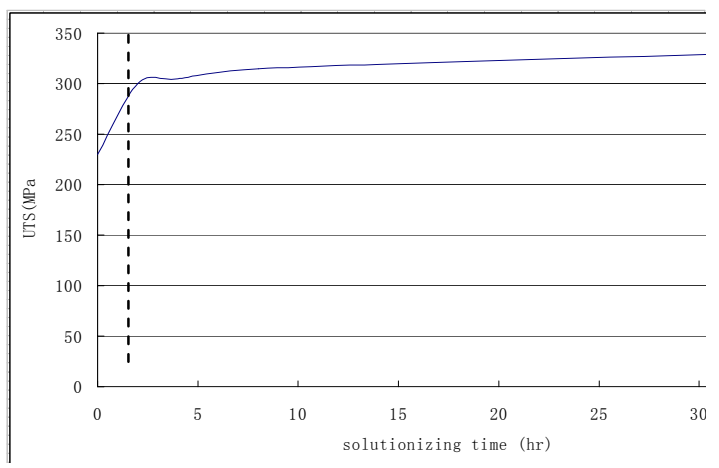

Figure 4.4-9. Variation of tensile strength during solutionizing

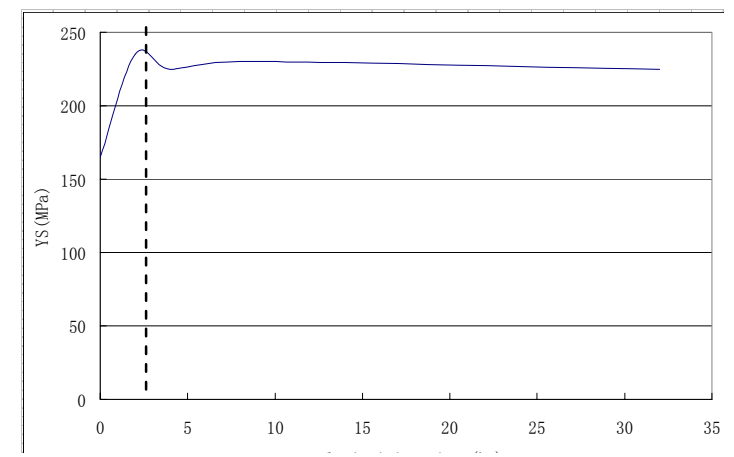

Figure 4.4-10. Variation of yield strength during solutionizing 


\section{Section 4: Integration of Thermal-Microstructure -Property Prediction}

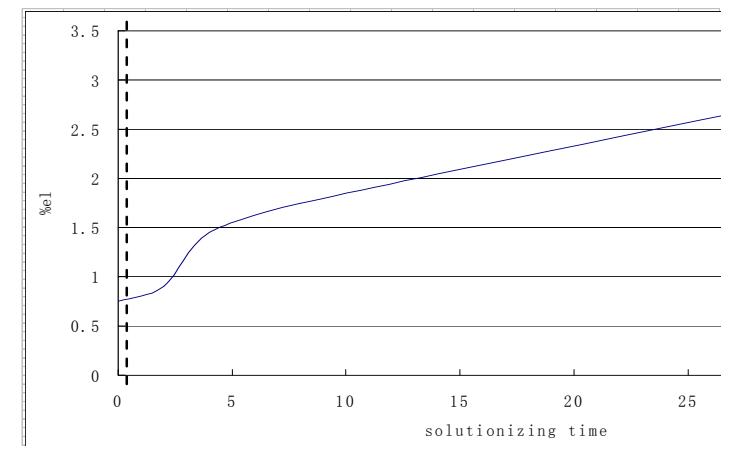

Figure 4.4-11. Variation of elongation during solutionizing 


\section{Section 5: Commercialization}

The software for microstructure evolution and property prediction developed under this DOE program will form a valuable add-on to commercial casting simulation software packages. Market entry should be encouraged.

We are currently working with the ESI Group to develop a commercialization plan for the models developed in this project. "ESI Group is the world leading organization for the numerical simulation of prototype and manufacturing process engineering in applied mechanics. The key to ESI Group's success is the use of realistic material physics, providing "as good as real" virtual solutions, in order to replace the lengthy trial and error processes on real prototypes. Calcom ESI, created in 1991, is an engineering company located in Lausanne, Switzerland which is involved in the development and marketing of the metallurgical simulation software ProCAST, PAM-QUIKCAST and CALCOSOFT. Foundries and cast houses use these software packages worldwide. Since 2003, Calcom ESI is owned by the international group ESI Group."

The models and software developed in this project are a good fit with ESI's approach to modeling and particularly the ProCAST software. A final commercialization plan should be developed in the next quarter. 


\section{Accomplishments}

\section{Publications \& conference proceedings}

[1] M. Qian, H. D. Brody, J. E. Morral, and D. Zhang, "Simulation of Phase Appearance, Phase Dissolution, and Solute Redistribution in Multicomponent Aluminum Casting Alloys," in ASM International Proceedings, Metals Park, Ohio, 2003, pp. 31-38.

[2] M. Fontecchio, M. Maniruzzaman, and R. D. Sisson, "Effect of Bath Temperature and Agitation Rate on the Quench Severity of 6061 Aluminum Quenched in Distilled Water," in the 13th Annual International Federation for Heat Treatment \& Surface Engineering (IFHTSE) Congress, Columbus, OH., 2002.

[3] J. Kang and Y. Rong, "Modeling And Simulation Of Heat Transfer In Loaded Heat Treatment Furnaces," in 13th Annual International Federation for Heat Treatment \& Surface Engineering (IFHTSE) Congress, Columbus, OH., 2002.

[4] J. C. C. M. Maniruzzaman, C. McGee, S. Ma and R.D. Sisson, "CHTE quench probe system - a new quenchant characterization system," in Proceedings of the 5th International Conference on Frontiers of Design and Manufacturing (ICFDM 2002), Dalian, China, 2002, pp. 619-625.

[5] M. Maniruzzaman and R. D. Sisson, "Investigation of bubble nucleation site density during quenching heat treatment process using video imaging," in the TMS Annual Meeting 2002, Seattle, WA., 2002.

[6] J. E. Morral, "Databases for Computational Thermodynamics and Diffusion Modeling," in NIST Workshop, Gaithersburg, MD., 2002.

[7] J. E. Morral, H. D. Brody, and D. Zhang, "Development of Process-Microstructure and Process-Property Maps for 319 Aluminum Casting Alloys," in the ASM Materials Solutions Conference and Show, Columbus, OH, 2002.

[8] M. Qian, H. D. Brody, J. E. Morral, and D. Zhang, "Simulation of Phase Appearance, Phase Dissolution, and Solute Redistribution in Multicomponent Aluminum Casting Alloys," in the 13th Annual International Federation for Heat Treatment \& Surface Engineering (IFHTSE) Congress, Columbus, OH., 2002.

[9] Y. Rong, R. D. Sisson, J. E. Morral, H. Brody, and J. Goldstein, "An Integrated Heat Treatment Model for Aluminum Castings," in the Semi-annual Meeting of the Center for Heat Treating Excellence (CHTE), Worcester, MA,USA., 2002. 


\section{Accomplishments}

[10] R. D. Sission, "Computer simulations and database needs for energy savings, zero environmental impact and quality improvements for the Heat Treating Industry," in NIST Workshop, Gaithersburg, MD., 2002.

[11] S. Varde, M. Takahashi, M. Maniruzzaman, and R. D. Sisson, "Web-based Data Mining for Quenching Analysis," in the 13th Annual International Federation for Heat Treatment \& Surface Engineering (IFHTSE) Congress, Columbus, OH., 2002.

[12] J. Kang and Y. Rong, "Modeling and simulation of heat transfer in loaded heat treatment furnaces," in International Surface Engineering Congress - Proceedings of the 1st Congress, 2003, pp. 337-343.

[13] J. Kang, R. Vaidya, R. Ourushothaman, W. Wang, and Y. Rong, "A Computer Aided Heat Treating Planning System," in ASM 22nd Heat Treating Conference \& 2nd International Surface Engineering Congress, Indianapolis, IN., 2003.

[14] S. Ma, M. Maniruzzaman, and R. D. Sisson, "The Effects of Quenching Rates on Phase Transformations in Age Hardenable Aluminum Alloy 2024," in 2003 ASM Materials Solutions Conference, Pittsburg, Pennsylvania, 2003.

[15] S. Ma, A. Varde, M. Takahashi, D. Rondeau, M. Maniruzzaman, and R. D. Sisson, "Quenching - Understanding, Controlling and Optimizing the Process," in 4th International Conference on Quenching and the Control of Distortion, Beijing, China, 2003.

[16] M. Maniruzzaman, M. Fontecchio, and R. D. Sisson, "Optimization of an Aluminum Alloy Quenching Process in Poly-Oxethylene Glycol Polymer Solutions using Taguchi Methods," in ASM 22nd Heat Treating Conference \& Exposition, Indianapolis, Indiana, 2003.

[17] M. Maniruzzaman, S. Ma, K. Bradshaw, L. Costesso, G. Holmes, and R. D. Sisson, "The Effects of Process Parameters on the Quenching Performance of 2024 Aluminum Alloy in Aqueous Solutions of PAG Polymers," in 2003 ASM Materials Solutions Conference, Pittsburg, Pennsylvania, 2003.

[18] M. Maniruzzaman and R. D. Sisson, "Heat Transfer Coefficients for Quenching Process Simulation," in the 2nd International Conference on Thermal Process Modelling and Computer Simulation, Société Française de Métallurgie et de Matériaux, Nancy, France, 2003. 


\section{Accomplishments}

[19] M. Maniruzzaman, M. B. Stratton, T. A. Rogers, L. P. Barber, and R. D. Sisson, "Effect of Agitation on the Quenching Performance of a Quench Oil using CHTE Quench Probe System," in MS\&T 2003 conference, Chicago, IL., 2003.

[20] M. Qian, S. Adibhatla, F. Yi, D. Zhang, X. Pan, H. D. Brody, and J. E. Morral, "Solute Redistribution during the Solution Treatment of Cast Al-Si-Cu-Mg-Fe Alloys," in ASM International Metals Park, OH., 2003.

[21] M. Qian, F. Yi, D. Zhang, X. Pan, H. D. Brody, and J. E. Morral, "Solute Redistribution and Phase Appearance in As-cast Al-Si-Cu-Mg-Fe Alloys," in ASM International Metals Park, OH., 2003.

[22] D. K. Rondeau, M. Maniruzzaman, and R. D. Sisson, "The Effects of Part Orientation on Heat Transfer during Quenching," in the 22nd Heat Treating Conference \& Exposition, Indianapolis, Indiana, 2003.

[23] K. Rong and D. Apelian, "Computerized Heat Treatment Planning System for Batch Furnaces," Industrial Heating, vol. 10, pp. 27-29, 2003.

[24] R. D. Sisson, M. Maniruzzaman, and S. Ma, "Strategies for Quenching Sensors and Control," in the 132nd TMS Annual Meeting, San Diego, CA., 2003.

[25] M. Takahashi, M. Maniruzzaman, and R. D. Sisson, "Visualizing the Evolutions of Microstructure through Quenching Process," in MS\&T 2003 conference, Chicago, IL., 2003.

[26] A. S. Varde, M. Maniruzzaman, and R. D. Sisson, "The QuenchMiner Expert System for Quenching and Distortion Control," in ASM 22nd Heat Treating Conference \& Exposition, Indianapolis, Indiana, 2003.

[27] A. S. Varde, M. Takahashi, E. A. Rundensteiner, M. O. Ward, M. Maniruzzaman, and R. D. Sisson, "QuenchMinerT: Decision Support for Optimization of Heat Treating Processes," in IEEE'S IICAI-03, the 1st Indian International Conference on Artificial Intelligence, Hyderabad, India, 2003.

[28] A. S. Varde, M. Takahashi, E. A. Rundensteiner, M. O. Ward, M. Maniruzzaman, and R. D. Sisson, "Knowledge Discovery and Data Visualization in Heat Treating of Materials," in the 15th International Conference on Scientific and Statistical Database Management (SSDBM), Cambridge, Massachusetts, USA., 2003. 


\section{Accomplishments}

[29] J. Yuan, J. Kang, Y. Rong, and R. D. Sisson, "FEM Modeling of Induction Hardening Processes in Steel," ASM Journal of Materials Engineering and Performance, vol. 12, pp. 589-596, 2003.

[30] J. Yuan, Y. Rong, and R. D. Sisson, "Finite Element Analysis of Induction Hardening Process of Steel," in ASM 22nd Heat Treating Conference \& 2nd International Surface Engineering Congress, Indianapolis, IN., 2003.

[31] J. Yuan, Y. Rong, and R. D. Sisson, "Development of a Computer Model for Induction Hardening of Steel," in ASME IMECE, Washington, DC., 2003.

[32] L. He, J. Kang, T. Huang, and Y. Rong, "The integrated technique for the heat treatment of aluminium-alloy castings: a review " HEAT TREATMENT OF METALS, vol. 31, pp. 69-72, 2004.

[33] J. Kang, K. Rong, and T. Huang, "Modeling and Simulation of Heat Transfer in Loaded Continuous Heat Treatment Furnace," in 14th International Federation for Heat Treatment and Surface Engineering Congress, Shanghai, China, 2004.

[34] J. Kang, K. Rong, and T. Huang, "Modeling and Simulation of Heat Transfer in Loaded Heat Treatment furnaces," in The Third Sino-Korean Conference on Advanced Manufacturing Technology, Xi'an, China, 2004.

[35] J. Kang, Y. Rong, and W. Wang, "Numerical simulation of heat transfer in loaded heat treatment furnaces," Journal of Physics, vol. 4, pp. 545-553, 2004.

[36] M. Maniruzzaman and R. D. Sisson, "Multiphase Flow Modeling of Quenching Heat Treatment Process," in Multiphase Phenomena in Materials Processing in 2004 TMS Annual Meeting, Charlotte, North Carolina, 2004.

[37] M. Maniruzzaman, M. Stratton, and R. D. Sisson, "Computer Simulation of High Pressure Gas Quenching Process," in Modeling and Computer Applications in Metal Casting, Shaping \& Forming Processes in MS\&T 2004, New Orleans, Louisiana, 2004.

[38] M. Maniruzzaman, M. Stratton, and R. D. Sisson, "Numerical Study of Fluid Flow in an Agitated Quench Tank," in CFD Modeling and Simulation of Engineering Processes in 2004 TMS Annual Meeting, Charlotte, NC., 2004.

[39] M. Maniruzzaman, J. Yuan, and R. D. Sisson, "Effect of Non-uniform Surface Heat Extraction on Dimensional Stability of a Quenched Part," in Modeling and Computer Applications in Metal Casting, Shaping \& Forming Processes in MS\&T 2004, New Orleans, Louisiana, 2004. 


\section{Accomplishments}

[40] X. M. Pan, C. Lin, J. E. Morral, and H. D. Brody, "An Assessed Thermodynamic Database for Al Alloy 519, contributed presentation," in ASM International Materials Solution Conference and Exposition, Columbus, Ohio., 2004.

[41] D. Rondeau, M. Maniruzzaman, M. Stratton, and R.D.Sisson, "Effect of Immersion Orientation and Non-uniform Fluid Flow on a Quenched Part during Liquid Quenching," in General session of MS\&T 2004 conference, New Orleans, Louisiana, 2004.

[42] A. Varde, E. Rundensteiner, M. Maniruzzaman, and R.D.Sisson, "Computational Estimation of Heat Transfer Curves for Microstructure Prediction and Decision Support," in 2004 MS\&T '04: Materials Science \& Technology 2004: Computational Microstructure Evolution in Steel, New Orleans, Louisiana, 2004.

[43] A. Varde, E. Rundensteiner, M. Maniruzzaman, and R. D. Sisson, "Augmenting MatML with Heat Treating Semantics," in ASM Materials Solutions Conference and Exposition: Materials Property Databases, Columbus, Ohio., 2004.

[44] H. D. Brody, "Influence of Solution Treatment on Microstructure and Properties of Aluminum Castings," in The Materials Science \& Technology + Heat Treatment Society 2005, Pittsburgh, PA., 2005.

[45] L. Lee, M. Maniruzzaman, and R. D. Sisson, "The effect of flow rate and droplet distribution on spray quenching performance of 4140 steel and IVF probe in water," in Materials Science \& Technology + Heat Treatment Society 2005, Pittsburgh, PA., 2005.

[46] S. Ma, M. Maniruzzaman, J. Chaves, and R. D. Sisson, "Modeling of the effects of surface oxides on the quenching rates of 4140 steel in commercial mineral oils," in The Materials Science \& Technology + Heat Treatment Society 2005, 2005.

[47] S. Ma, M. Maniruzzaman, and R. D. Sisson, "Model Development of Quench Factor Analysis and Microstructure Prediction of Cast Al-Si-Mg Alloys," in The Materials Science \& Technology + Heat Treatment Society 2005, Pittsburgh, PA., 2005.

[48] S. Ma, M. Maniruzzaman, and R. D. Sisson, "Application of Jominy End Quench Approach for the Development of Quench Factor Analysis and Microstructure Prediction of Cast Al-Si-Mg alloys," in Solid-Solid Phase Transformations in Inorganic Materials 2005 (TMS), Phoenix, Arizona., 2005.

[49] M. Maniruzzaman, Y. Rong, and R. D. Sisson, "Optimization of solution treatment process for lost foam casting A356 aluminum alloy engine heads," in Materials Science \& Technology + Heat Treatment Society 2005, Pittsburgh, PA., 2005. 


\section{Accomplishments}

[50] X. M. Pan, C. Lin, J. E. Morral, and H. D. Brody, "An assessment of thermodynamic data for the liquid phase in the Al-rich corner of the Al-Cu-Si system and its application to the solidification of a 319 alloy," Journal of Phase Equilibria and Diffusion, vol. 26, pp. 225-233, June 2005.

[51] Y. Zhou, J. Kang, Y. Rong, Y. Feng, and H. D. Brody, "Integrated Numerical Model for Aluminum Alloy Solutionizing: Thermal-Microstructure-Property Simulation and Process Optimization," in Heat Treat Theory \& Innovation Program - Modeling Heat Treating Processes 2005 at the 23rd ASM Heat Treating Society Conference and Exposition, Pittsburgh, PA., 2005.

[52] J. Kang and Y. Rong, "Modeling and simulation of load heating in heat treatment furnaces," Journal of Materials Processing Technology, vol. 174, pp. 109-114, May 26 2006.

[53] Y. Zhou, J. Kang, and Y. Rong, "Optimization of Load Arrangement Design and Thermal Recipe Design for Time and Energy Efficient Heat Treatment Processes," in Simulation of Aluminum Shape Casting Processing: From Alloy Design to Mechanical Properties (TMS), San Antonio, CA., 2006. 


\section{Accomplishments}

\section{Software package developed through the project}

In this project, modules of the integrated system of software, databases, and design rules have been developed, verified and ready to be marketed to enable quantitative prediction and optimization of the heat treatment of aluminum castings to increase quality and productivity, reduce the heat treatment cycle times and reduce energy consumption. The system structure and interface are displayed as following figures.

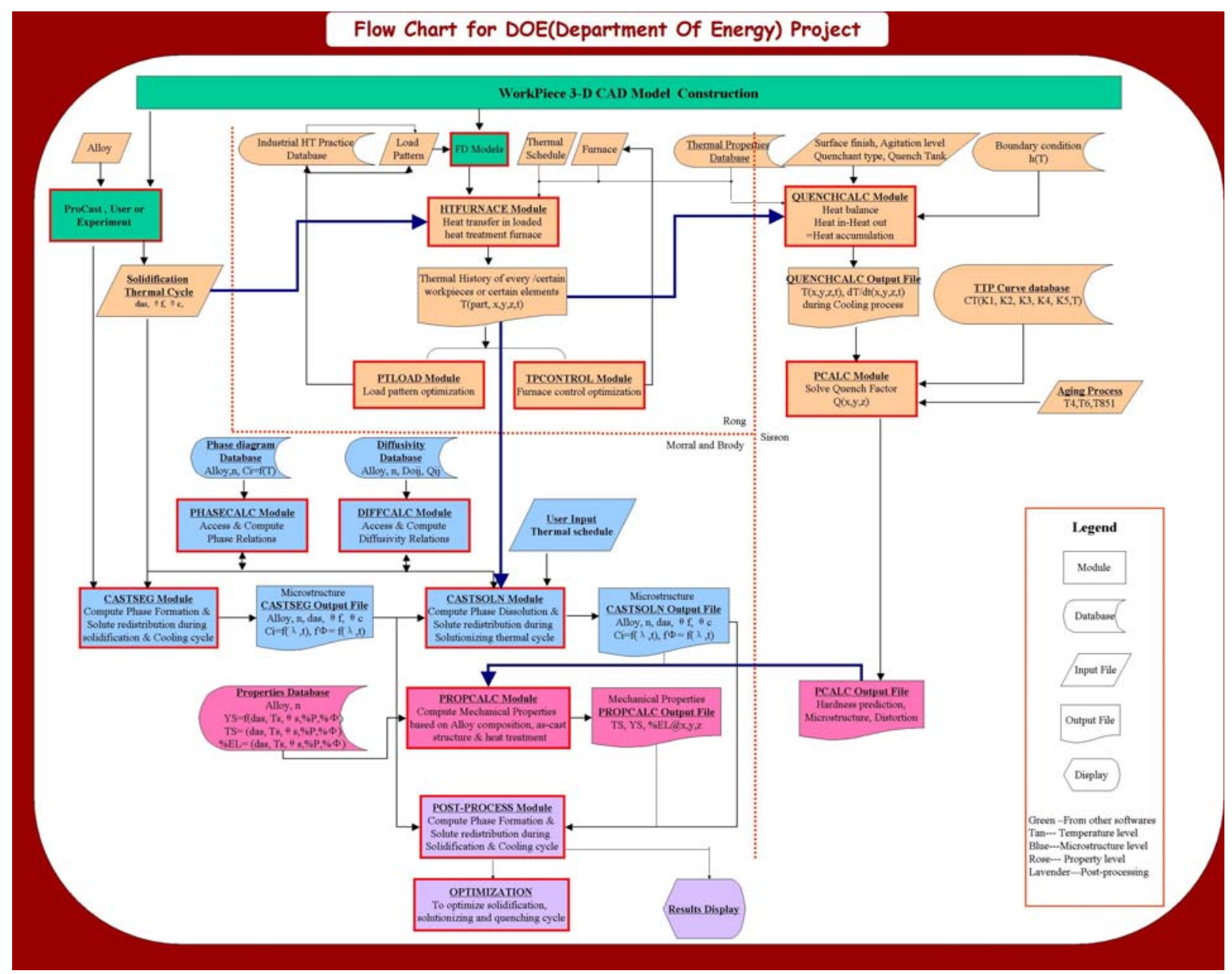

Figure A-1. Structure design of the system 


\section{Accomplishments}

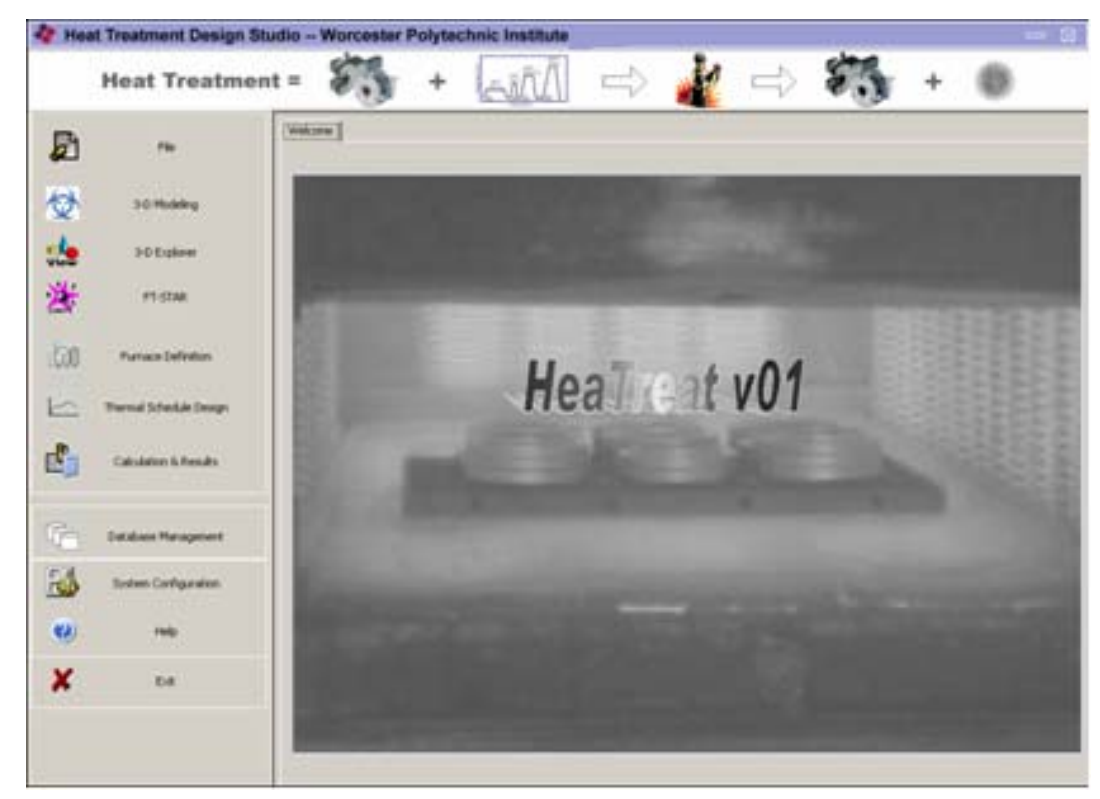

Figure A-2. The system interface

The software can be used to predict the thermal cycle in critical locations of individual components in a furnace, the evolution of microstructure, and the attainment of properties in heat treatable aluminum alloy castings. The model takes into account the prior casting process and the specific composition of the component. The heat treatment simulation modules were designed to be used in conjunction with software packages for simulation of the casting process. The system was built upon a quantitative understanding of the kinetics of microstructure evolution in complex multicomponent alloys, on a quantitative understanding of the interdependence of microstructure and properties, on validated kinetic and thermodynamic databases, and validated quantitative models. 


\section{Conclusions}

In this project, modules for an integrated system of software, databases, and design rules has been developed, verified and ready to be marketed to enable quantitative prediction and optimization of the heat treatment of aluminum castings to increase quality and productivity, reduce the heat treatment cycle times and reduce energy consumption.

The heat transfer in loaded heat treatment furnace is solved by a set of models, including radiation, conduction, convection and combustion, based on finite difference method. Experiments were carried out to validate the system by the cylindrical workpiece similar to the quenching probe. The calculated temperature results matches the measured results very well during the period without furnace temperature control region.

Methodologies for optimizing load arrangement and thermal cycle so as to reduce cycle time and energy consumption were discussed and applied in the system. By comparing the relative cost of possible load arrangement designs, the optimum load arrangement in the furnace for a combined consideration of time, energy and quality can be determined. The modified thermal cycle with the proposed algorithm to avoid load overheating not only shortens cycle time but also reduces energy consumption while ensuring quality.

Finally, a complete thermal-microstructure-property process model and the simulation software for aluminum solutionizing have been developed. It has the capability to model the entire furnace-load system with arbitrary shape in 3-D, and to take into account the effect of as-cast condition of the alloy. The simulations and experiments of a laboratory scale case study basically match each other. The simulation results have shown great potential in shortening heating and soaking times, without compromising product quality. Better performance can be expected from the simulation if the calculation effectiveness is further enhanced.

Software to simulate microstructure evolution and solute redistribution in multicomponent and multiphase aluminum casting alloys in response to casting and solution heat treatment process parameters have been developed based on validated models, understanding, and databases. The 


\section{Conclusions}

validated databases for multicomponent alloys and predictive models enable comparable results to be achieved for a wide range of alloys and applications.

A simple to calibrate and simple to implement design rule has been demonstrated to predict tensile strength of aluminum casting alloys to as-cast microstructure and solution treatment time.

The software can be used to predict the thermal cycle in critical locations of individual components in a furnace, the evolution of microstructure, and the attainment of properties in heat treatable aluminum alloy castings. The model takes into account the prior casting process and the specific composition of the component. The heat treatment simulation modules were designed to be used in conjunction with software packages for simulation of the casting process. The system was built upon a quantitative understanding of the kinetics of microstructure evolution in complex multicomponent alloys, on a quantitative understanding of the interdependence of microstructure and properties, on validated kinetic and thermodynamic databases, and validated quantitative models. 


\section{References}

1. $\quad$ Bejan, A., ed. Heat transfer. 1984, John Wiley \& Sons: New Yok.

2. Seborg, D.E., T. F. Edgar, and D. A. Mellichamp., ed. Process Dynamics and Control. 1989, John Wiley\& Sons: New York.

3. Ross, P.J., Taguchi Techniques for Quality Engineering Second ed. 1996, McGraw-Hill: New York.

4. Fluent, Inc. [cited; Available from: http://www.fluent.com/solutions/whatcfd.htm.
.

5. Zhang, D.L. and L. Zheng, The Quench Sensitivity of Cast Al-7 Wt Pct Si-0.4 Wt Pct Mg Alloy. Metallurgical and Materials Transactions, 1996. 27A: p. 3983-3991.

6. Bates, C.E. and G.E. Totten. Application of Quench Factor Analysis to Predict Hardness under Laboratory Production Conditions. in First International Conference on Quenching \& Control of Distortion. 1992. Chicago, IL.

7. Staley, J.T., Quench Factor Analysis of Aluminum Alloys. Materials Science and Technology, 1987. 3(11): p. 923-935.

8. Staley, J.T. and M. Tiryakioglu. Use of TTP Curves and Quench Factor Analysis for Property Prediction in Aluminum Alloys. in Materials Solutions Conference. 2001. Indianapolis, IN: ASM International.

9. MacKenzie, S., Quench rate and aging effects in Al-Zn-Mg-Cu aluminum alloys, in Metallurgical engineering. 2000, University of Missouri-Rolla.

10. Dorward, R.C., A Dynamic Quench Model for Strength Predictions in Heat-Treatable Aluminum Alloys. Journal of Materials Processing Technology, 1997. 66: p. 25-29.

11. Rometsch, P.A., M.J. Starink, and P.J. Gregson, Improvements in Quench Factor Modelling. Materials Science and Engineering A, 2003. 339: p. 255-264.

12. Dolan, G.P., J.S. Robinson, and A.J. Morris. Quench Factors and Residual Stress Reduction in 7175-T73 Plate. in Materials Solutions Conference. 2001. Indianapolis, IN: ASM International.

13. MacKenzie, D.S. and J.W. Newkirk. Quench Factor Analysis for Heat Treatment Optimization of $7 X X X$ Aluminum Alloys. in International Federation of Heat Treatment and Surface Engineering Conference. 2001. Dubrovnik, Croatia. 


\section{References}

14. Bernardin, J.D. and I. Mudawar, Validation of the Quench Factor Technique in Predicting Hardness in Heat Treatable Aluminum Alloys. International Journal for Heat Mass Transfer, 1995. 38(5): p. 863-873.

15. Flynn, R.J. Precipitation Kinetics and the Time Temperature Property Curves of 7010. in Materials Solutions Conference. 2001. Indianapolis, IN: ASM International.

16. Staley, J.T., R.D. Doherty, and A.P. Jaworski, Improved Model to Predict Properties of Aluminum Alloy Products after Continuous Cooling. Metallurgical Transactions, 1993. 24A(November): p. 2417-2427.

17. Tiryakioglu, M. and J. Campbell, On Macrohardness testing of Al-7wt\%Si-Mg alloys I. Geometrical and mechanical aspects. Materials Science and Engineering A, 2003. 361: p. 232-239.

18. Chaudhury, S.K., L. Wang, and D. Apelian. Fluidized Bed Reactor Heat Treatment of A356 Alloy: Microstructure Analysis and Mechanical Properties. 2004: American Foundry Society.

19. Li, R.X., et al., Age-Hardening Behavior of Cast Al-Si Base Alloy. Materials Letters, 2004. 58: p. 2096-2101.

20. Zanada, A. and G. Riontino, A Comparitive Study of the Precipitation Sequences in Two AlSi7Mg Casting Alloys and their Composites Reinforced by $20 \% \mathrm{Al}_{2} \mathrm{O}_{3}$ Discontinuous Fibers. Materials Science Forum, 2000. 331-337: p. 229-234.

21. Rometsch, P.A. and G.B. Schaffer, Quench Modelling of Al-7Si-Mg Casting Alloys. International Journal for Cast Metals Res., 2000. 12: p. 431-439.

22. Maniruzzaman, M. Optimization of solution treatment process for lost foam casting A356 aluminum alloy engine heads. in Materials Science \& Technology + Heat Treatment Society 2005. 2005. Pittsburgh, PA: ASM International.

23. Peterson, N.L., Rothman, S.J., Impurity Diffusion in Aluminum. Phys.Rev., 1970. B1: p. 3264-3273.

24. Youdelis, W.V., Colton, D.R. and Calhoon, J., Can. J., On the Theory of Alloy Solidification in a Magnetic Field.Phys., 1964. 42: p. 2217.

25. Minamino, Y., Yamane, T. and Takahashi, T., Interdiffusion in the Aluminum-Rich Solid Solution of Al-Cu Alloys. J. Mater. Sc. Letters., 1985. 4: p. 797-798. 


\section{References}

26. Fujikawa, S.-I., Hirano, K-I. and Fukushima, Y., Diffusion of Silicon in Aluminum. Metall. Trans., 1978. 9A: p. 1811-1815. 